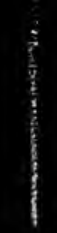

*

8

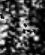

(3)

Thes 


\section{Ired Wilcox.

\section{THE LIBRARY OF THE UNIVERSITY OF CALIFORNIA DAVIS}

GIFT OF

ROBERT I. TENNEY 
$P \amalg G=23$ 



\section{QUANTITATIVE ANALYSIS}




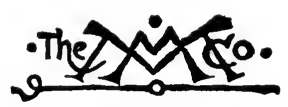

THE MACMILLAN COMPANY NEW YORK - BOSTON - CHICAGO DALLAS - ATLANTA - SAN FRANCISCO

MACMILLAN \& CO., LIMITED LONDON - BOMBAY - CALCUTTA MELBOURNE

THE MACMILLAN CO. OF CANADA, LTD. TORONTO 


\title{
A COURSE OF INSTRUCTION IN
}

\section{QUANTITATIVE \\ CHEMICAL ANALYSIS}

FOR BEGINNING STUDENTS

WITH EXPLANATORY NOTES, QUESTIONS AND ANALYTICAL PROBLEMS

\author{
BY' \\ GEORGE McPHAIL SMITH \\ PROFESSOR OF CHEMISTRY IN THE UNIVERSITY OF WASHINGTON
}

REVISED EDITION

Setw Bork

THE MACMILLAN COMPANY

1922

All rights reserved 
Copyright, rgig AND rg2t,

By THE MACMILLAN COMPANY.

Set up and electrotyped. Published July, I9I9.

Revised edition, Published October, I92I.

PRINTED IN THE UNITED STATES OF AMERICA 


\section{PREFACE}

THIs book is intended for use with classes consisting of students who have completed a substantial year's work in Elementary Chemistry and Qualitative Analysis, and who are beginning the study of Quantitative Analysis. On the laboratory side its main object is to furnish the student with directions sufficiently detailed to enable him to do successful work with a minimum of personal oversight from the instructor. The instructor is thereby placed in a position, in the laboratory, as well as in the classroom, to exert his efforts more especially towards the development in his students of theoretical knowledge and the power to think.

For the best results, the laboratory work should of course be accompanied by lectures and recitations; and, with the latter in mind, it has seemed desirable to include the questions of Part IV, and the stoichiometrical problems of Part V. 'The questions of Part IV are for the most part answered in the notes or elsewhere in the book; but it has been the writer's experience that beginners react more favorably to concrete questions assigned in advance for study, than to the same questions when put to them for the first time just after they are supposed to have mastered the details and principles of a specific analytical process. The problems of Part $\mathrm{V}$ are such as are regularly met with in analytical work, and their conscientious study will give the student an insight into the principles of a wide variety of processes; the answers to the problems have been intentionally omitted. It has been the writer's practice to require, as a written exercise to be handed in at the beginning of a recitation, the solution of a definite number of problems each week throughout the course; these to be graded and returned at the end of the following recitation.

Part I comprises a somewhat detailed discussion of those principles, both of theory and practice, which should be constantly kept in mind by the worker in analytical chemistry. It is realized that the student can assimilate this material only gradually, as his progress advances; 
but the writer feels strongly that such a discussion should be available, especially in an introductory manual.

The gravimetric and volumetric processes detailed in Parts II and III are such as have for several years been included in the elementary courses of quantitative analysis at the University of Illinois. On the basis of a good deal of experience, they are regarded as processes which are well adapted to the progressive instruction of beginners, and are believed to constitute an excellent foundation for the continued study of analytical chemistry. Also, it is believed, they are sufficiently varied to meet the needs of students who will not extend their study beyond the period of an introductory course.

In addition to the help derived from other books and from journal articles, in the preparation of this manual, the writer wishes to acknowledge his indebtedness to the following works on analytical chemistry: W. C. Blasdale's Principles of Quantitative Analysis; A. Fischer's Elektroanalytische Schnellmethoden; W. F. Hillebrand's The Analysis of Silicate and Carbonate Rocks; E. G. Mahin's Quantitative Analysis; J. W. Mellor's Treatise on Quantitative Inorganic Analysis; H. P. Talbot's Quantitative Chemical Analysis; and F. P. Treadwell's Lehrbuch der analytischen Chemie.

G. McP. SмIтH.

SeAttle, WashingtoN 


\section{CONTENTS}

\section{PART I \\ INTRODUCTION}

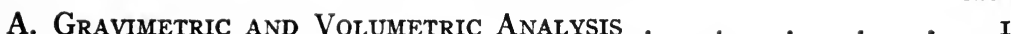

B. General Remarks Concerning Quantitative Work • • 3

Physical Conditions; Reagents and Glassware; Utilization of

Time; Accuracy; Laboratory Records.

C. The Operations of Analytical Chemistry

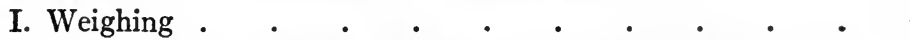

Standards of Mass; The Balance; The Use and Care of the Analytical Balance; Determination of the Restpoint; Methods of Weighing; The Calibration of a Set of Weights; Errors Due to Inequalities in Length in the Beam Arms; Errors Due to Atmospheric Buoyancy; Summary.

II. Precipitation

Qualities Desirable in Precipitates Which Are to be Used in Gravimetric Determinations; Colloidal and Finegrained Precipitates; The Contamination of Precipitates; The Theory of Precipitation.

III. Filtration and the Washing of Precipitates

The Selection and Use of Paper Filters; Wash Bottles;

Gooch's Filtration Crucible; The Theory of Washing Precipitates.

IV. The Drying and Ignition of Precipitates . . . . $\quad 38$

Drying Ovens; Desiccators; Crucibles.

V. The Evaporation of Liquids $\quad$ • $\quad$. $\quad . \quad 4^{2}$

VI. The Volumetric Measurement of Liquids . . . 43

Volumetric Apparatus; Sources of Error in the Use of

Volumetric Apparatus; The Calibration of Volumetric Apparatus.

D. The Preparation of Samples for Analysis . . . . 50 


\section{PART II}

\section{GRAVIMETRIC ANALYSIS}

EXERCISES WITH THE BALANCE..$\cdot \cdot{ }^{-} \cdot{ }^{-}$

The Determination of Chlorine in a Soluble Chloride. • 55

The Determination of Iron and of Sulphur in a Soluble SulPHATE OP IRON .

The Determination op Sulphur in a Sulphide Ore • • $\quad$. 66

The Determination of Potash in Soluble Salts • • • 68

The Determination of Carbon Dioxide in Limestone • • 7 I

The Determination of Calcium and Magnesium Oxides in Lime-

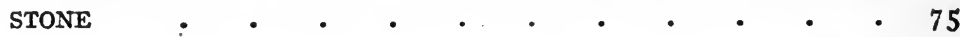

The Determination of Phosphoric Anhydride in Phosphate ROCK . . • • • . • • • • • $8 \mathrm{I}$

The Determination of Silica in a Refractory Silicate • • 85 The Electrolytic Determination of Copper • • • • • $\quad 39$

\section{PART III}

\section{VOLUMETRIC ANALYSIS}

General Discussion $• \quad \cdot \quad \cdot \quad \cdot \quad \cdot \quad \cdot \quad \cdot \quad \cdot$ ・

Fundamental Principles; The Fundamental Reactions of Volumetric Analysis; Determination of the End-Point; General Theory of Indicators; Volumetric vs. Gravimetric Methods; General Remarks.

A. Neutralization Methods: Acidimetry aNd Alkalimetry . IO7

Fundamental Principles; Methyl Orange Solution; Phenolphthalein Solution; Standard Acid Solutions; Standard Alkali Solutions.

The Preparation and Standardization of Approximately HALF-NORMAL Hydrochloric ACID AND SODIUM HydroXIDE - I I 2 The Determination of the Alkaline Value of Soda - . ir6 The Determanation of the Available Hydrogen-ion in an ACID 
The Determination of Protein Nitrogen by the Kjeldahl METHOD • • • • • . . . . . . . $\mathrm{I} 20$

B. Methods of Oxidation and Reduction • • . . . I 24

Standard Solutions; Indicators.

i. Dichromate Processes . . . . . . . . I25

Fundamental Principles.

The Preparation and Standardization of Approximately

Tenth-normal Dichromate and Ferrous Iron SoluTIONS

The Determination of Iron in Siderite • • • . I 29

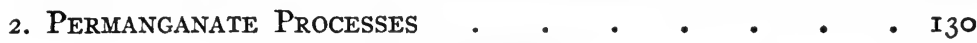

Fundamental Principles.

The Preparation and Standardization of an Approximately Tenth-normal Solution of Potassium Per-

MANGANATE $\cdot$ •

The Determination of Iron in Hematite . • • - I33

The Determination of Calcium in Limestone . • . I37

The Determination of the $\mathrm{MnO}_{2}$-Value of Pyrolusite . I 38

The Determination of Phosphorus in Steel . • . I 40

The Determination of Manganese in an Ore . • - I44

3. Iodometric Processes . $\cdot$. . . . . . I48

Fundamental Considerations; Determination of the

End-Point; Preparation of Starch Solution.

The Preparation and Standardization of Approximately

Tenth-normal Solutions of Iodine and Sodium Thio-

SULPHATE • • • • • • • • • • • • I5I

The Determination of Antimony in Stibnite . • . I55

The Determination of Chromium in Chromite • - I57

The Determination of Lead in an Ore • • . I59

The Determination of Copper in an Ore . . . i62

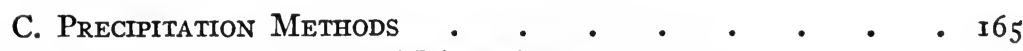

General Discussion.

The Preparation and Standardization of Approximately

Tenth-normal Solutions of Silver Nitrate and Am-

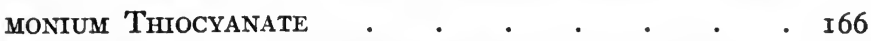

The Determination of Chlorine in a Soluble Chloride 168 


\section{PART IV}

QUESTIONS

\section{PART V}

\section{ANALYTICAL PROBLEMS}

Preliminary Discussion: The Solution of Typical Problems . 185 Problems

- 192

\section{APPENDIX}

Preparation of the Reagents

Sulphuric Acid-Dichromate Cleaning Solution . . . 208

Analytical Samples for the Use of Students . . . . 208

Apparatus in the Student's Desk . . . . . . . 209

LOGARITHMS . . . • . . . . . . . 210

ANTILOGARITHMS $. \quad . \quad . \quad . \quad . \quad . \quad . \quad . \quad . \quad$. 212

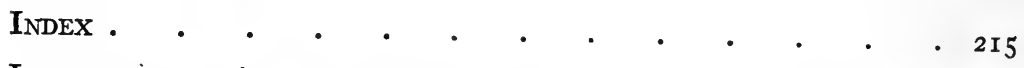

International Atomic Weights, I92I . . . Back Cover Sheet 


\section{PART I}

\section{INTRODUCTION}

\section{A. GRAVIMETRIC AND VOLUMETRIC ANALYSIS}

QuANTITATIVE analysis has for its object the determination of the quantities of the elements or compounds which are present in particular samples of material. The results are usually expressed in terms of percentage, ordinarily by weight; but sometimes, as in the analysis of gases, by volume.

The procedure to be employed in a specific case will often depend upon the qualitative composition of the sample. A qualitative analysis, therefore, should precede a quantitative, unless the composition of the sample is sufficiently well known.

In the performance of quantitative determinations there are two principal methods of procedure, according to which the subject is subdivided into gravimetric and volumetric analysis. In addition, there are gasometric methods, and various physical methods, of analysis; but these will not be described in this book.

In a gravimetric analysis, a weighed sample is taken, and the substances to be determined are separated, one after another, either in the free state, or in the form of suitable compounds. Each final product is weighed, and, from its weight, the weight, and therefore the percentage, of the corresponding substance in the sample can be calculated.

The substance to be weighed is in most cases separated from solution by precipitation, though in many instances it is deposited upon a weighed cathode or anode by electrolysis. Sometimes it is separated from other substances by extraction with a solvent, and sometimes in the form of a gas, the weight of the 
gas being determined either by absorbing it in a weighed quantity of some substance and noting the increase, or by noting the decrease in weight due to the removal of the gas alone.

In a volumetric analysis a weighed sample is also taken, but the quantity of the substance to be determined is arrived at by causing a definite reaction to take place, the reagent being added from a burette, as a solution of known concentration. This operation is called titration. From the volume of the solution added, it is easy to calculate the weight of the substance present in the sample.

In many instances, it is necessary in volumetric analysis also to separate the substance to be determined from interfering substances present with it in the sample; but, instead of finally weighing it, the substance is again brought into solution, in suitable form, and its quantity estimated by titration.

In order to illustrate the two methods, let us consider the determination of silver in a silver coin.

(a) Gravimetric Method. The weighed sample is dissolved in nitric acid, the solution diluted, and the silver separated from copper, by precipitation as insoluble silver chloride, with dilute hydrochloric acid. The precipitate is filtered off, washed, dried, and weighed. From its weight, the weight of silver is calculated, as follows :

$$
\frac{\mathrm{Ag}}{\mathrm{AgCl}} \times \text { wt. of precipitate }=\text { wt. of silver. }
$$

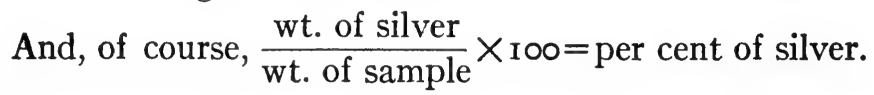

(b) Volumetric Method. The weighed sample is dissolved in nitric acid, diluted as before, and the silver converted into the insoluble chloride by the gradual addition, from a burette, of a solution of sodium chloride of known concentration. As soon as, after stirring each time and allowing the precipitate to settle, the first drop is added which fails to induce further precipitation, the reaction is known to be complete; and the number of cubic centimeters required, multiplied by the silver equivalent 
of the sodium chloride solution per cubic centimeter, gives directly the weight of silver in the sample.

\section{B. GENERAL REMARKS CONCERNING QUANTITATIVE WORK}

Physical Conditions. The inside and top of the desk, the reagent bottles, and all apparatus should be kept clean and ready for use. Vessels for use in analytical work should be perfectly clean, and the outside of glassware should be wiped dry before use, especially if it is to be heated over a burner, or on the hot plate. Flasks, funnels, etc. may be freed from inside grease films by rinsing, or by longer contact, with either a solution of sodium hydroxide in alcohol, or with sulphuric acid-dichromate cleaning solution. (For the preparation of the latter, see the Appendix.)

Reagents and Glassware. One of the greatest hindrances to exact work lies in the difficulty of securing satisfactory reagents. And, in addition to this, much of the glassware on the market is of an inferior grade, and not at all suited for analytical work.

The habit of carefully testing reagents, including distilled water, cannot be too early acquired; constant vigilance should be exercised in guarding against the presence of impurities which would vitiate the work under way. As is generally known, a "C. P." label is no guaranty whatever of the purity of a reagent, and the "guaranteed" or " analyzed" reagents, sold at higher prices, are at times inferior to products for which no special claim is made.

Very pure acids are obtainable in the market, and, in general, these need no redistillation. But, owing to its basic nature, ammonia ought to be redistilled at short intervals, after a preliminary treatment with slacked lime to remove absorbed carbonic acid; to prevent contamination of the solution through its solvent action upon glass, stock bottles for ammonia may be coated inside with ceresin. 
Owing to the solvent action on glass of many solutions of solid reagents, these should be prepared frequently and in small quantities; or, better, the solid should be dissolved as wanted. This applies particularly to such reagents as ammonium oxalate, microcosmic salt and sodium phosphate, alkaline magnesia mixture, ammonium carbonate, etc.

The stopper of a reagent bottle should always be held in the fingers until returned to the bottle. This will prevent contamination, whether due to an interchange of stoppers, or to some other cause. The contents of such bottles must of course be protected at all times from contamination.

Utilization of Time. Economy of time in the laboratory is best insured through a preliminary study of the work in prospect, followed by a plan for its prompt execution. It may be stated in general that several analyses should always be under way, and that no vessel should ever be left unlabelled. ${ }^{1}$ In this way, the analyst will always have something to occupy his time. At the outset, of course, the student cannot be expected to approach this degree of efficiency; nevertheless, even here, time can be either wasted or utilized,-e.g. the student should bear in mind that the time required for the solution of several samples, or for the filtration or evaporation of several solutions, is the same as that required in the case of a single one.

Accuracy. Quantitative work, to be successful, must be performed without the slightest loss of material, as well as without gain of material from the outside. Whenever solutions are to be boiled, therefore, or in dissolving substances for analysis, especially if finely divided, or if effervescence is at all likely, the vessels should be kept covered; also, in the evaporation of liquids, even on the steam bath, large watch glasses should be supported above the vessels, in order to prevent the entrance of dust, while at the same time allowing the vapor to escape freely.

1 In certain cases, it is customary to keep the record in terms of serial numbers. Platinum crucibles, for example, should have stamped upon them serial numbers, and under these numbers records should be kept in the notebook of their contents. 
It is of course indispensable that the analyst should be possessed of a certain degree of dexterity in the performance of the quantitative operations. But, while a few persons seem to have been born with this skill, the majority can acquire it only through careful and persistent application. The student who finds himself at first unable to do good work, therefore, should not try to hide the fact, but should patiently endeavor to overcome the handicap; nothing can be more fatal to successful scientific work than a lack of personal integrity.

All determinations should be made in duplicate, and in general the results should closely agree. "Check results," however, do not necessarily indicate accuracy; they depend very largely upon identical conditions in the two analyses, and are possible even in the case of inaccurate methods. A failure to obtain check results, however, is conclusive in its testimony; one result is certainly wrong, and the other is little worthy of confidence.

It is often thought that no operation in an analysis need be carried out with more exactitude than the one which necessarily involves the greatest error; that if a given step cannot be executed without an uncertainty of $0.1 \%$, it would be useless to avoid errors of that magnitude in other stages of the process. If carried to its logical conclusion, this would imply that a method capable of furnishing results within, say, 0.15\% of the true value, might just as well be allowed to yield results a few tenths of a per cent farther off. Of course, a compensation of errors might take place, but this is not always even probable. Nevertheless, if an error of, say $0.15 \%$ cannot be avoided, it would be inadvisable to devote much labor towards the reduction of a very much smaller individual error also involved in the process.

Laboratory Records. Notebooks should contain the analytical data, systematically entered at the time of observation, and, in addition, an account of any unexpected development in the analysis. Since a neat and intelligible record is of the 
greatest importance, the following sample page is offered in the way of suggestion. In the calculation of the result from the analytical data, it is useless to proceed more than one place beyond the last that may reasonably be considered correct; according to the data in this case, it is not certain whether the first figure after the decimal should be 4, 3, or 2, - nothing, therefore can be known concerning the second figure, and it is not included in the report of the mean.

Determination of Chlorine in a Soluble Chloride - Sept. 21-28

\begin{tabular}{|c|c|c|}
\hline & I & II \\
\hline Sample Tube, etc. & 8.4237 & 8.2377 \\
\hline Tube minus Sample & 8.2377 & 8.org8 \\
\hline Wt. of Sample & $\overline{0.1860}$ & $\overline{0.2179}$ \\
\hline Wt. of Crucible No. & (7) $5 \cdot 35^{88}$ & (8) 5.0072 \\
\hline \multirow{2}{*}{$\begin{array}{r}\text { Crucible }+\mathrm{AgCl}, \text { Ist time } \\
\text { 2d time }\end{array}$} & 5.7830 & $5 \cdot 5024$ \\
\hline & 5.7828 & 5.5023 \\
\hline Wt. of Crucible & $5 \cdot 35^{88}$ & 5.0072 \\
\hline Wt. of $\mathrm{AgCl}$ & $\overline{0.4240}$ & $\overline{0.4951}$ \\
\hline Per cent of Chlorine & 56.40 & 56.21 \\
\hline
\end{tabular}

Mean Value $=56.3 \%$

\section{THE OPERATIONS OF ANALYTICAL CHEMISTRY}

The chief operations involved in analytical work which can be profitably discussed at this point are weighing, precipitation, filtration, and the washing of precipitates, the drying and igmition of precipitates, the evaporation of liquids, and the volumetric measurement of liquids.

These operations will be described in the following sections, which should be studied carefully by the beginner. The student should from the start try to appreciate the importance of paying great attention to details and of closely observing the conditions necessary for the greatest accuracy. 


\section{WEIGHING ${ }^{1}$}

Standards of Mass. The fundamental standard of mass adopted by the United States is a cylinder of platinum-iridium kept at the International Bureau of Weights and Measures near Paris. Two authentic copies of this standard, of the same form and composition, are kept in a vault at the National Bureau of Standards, and they are used only when needed to verify the secondary standards of the Bureau.

Mass standards are usually called "weights," but it should be realized that, while the weight of a body depends upon its attraction by the earth, the mass of a body is a property inherent in the body itself. Since, however, the masses of bodies are proportional to their weights at the same locality, we may compare the masses by making a comparison of the respective forces of gravity on the bodies under consideration. The purpose of weighing, then, is to compare the quantity of matter in a specific object with the quantity of matter in a given standard - a gram or kilogram weight. The comparison is made on the balance, by means of the lever principle, by suspending the object at one end of a beam, and the weights at the other end of the beam, the beam being virtually a kind of lever.

The Balance. The beam of the balance is supported on a central knife-edge, usually of agate, which rests upon a plane agate plate; and two pans for supporting the masses to be compared are vertically suspended from stirrups, each of which has an agate bearing which rests on a knife-edge fixed at one extremity of the beam. The arms of the balance are so graduated that a rider (of known weight) can be placed on the beam at any required distance from the central knife-edge.

If the three knife-edges are allowed to press continually upon their agate bearings, they soon become blunted, and wear furrows in the bearings. In order to prolong the life of the knife-

1 For more detailed information on the subject of weights and weighing, the student is referred to Circular of the Bureau of Standards, No. 3 (3d edition, r9r8), from which much of the following is taken. 
edges and bearings, the balance is provided with a "release" which separates the knife-edges from their bearings when the balance is not in use. If the balance shows signs of stiffness in the motion of beam and pans, the fault should be investigated at once. The defect may be due to an accumulation of dust between the knife-edges and their bearings; to the blunting of the knife-edges; or to the wearing of furrows in the bearings. To prevent the accumulation of dust, and also to prevent the interference of air currents while weighing, the balance is inclosed in a glass case.

In order to render small movements of the beam perceptible, there extends downwards from its center a long pointer which multiplies the rotational displacement. When equilibrium is established, the lower end of the pointer should come to rest in front of the zero of a scale which is located immediately behind this end.

The conditions which must be satisfied by a good balance are : (I) The balance must be consistent. It must give the same result in successive weighings of the same body. This condition depends upon the trueness of the knife-edges. (2) The balance must be accurate. At rest the beam must be horizontal when the pans are empty, and when equal weights are placed upon the pans. This condition depends upon the equality of the two arms. (3) The balance must be stable. The beam after being displaced from its horizontal position must return to its horizontal position. This condition depends upon the adjustment of the center of gravity. (4) The balance must be sensitive. It must show even a very small inequality in the two masses on the scale pans. This condition depends largely upon the length of the arms. (5) The balance must oscillate with reasonable rapidity. Short beams oscillate more rapidly than long ones.

The analytical balance will perform excellent service under the proper conditions, but great care in its use is essential if its accuracy is to be relied upon. It should be located in a room 
that is free from dust and fumes, and should stand upon a support that is free from shocks and vibrations.

The Use and Care of the Analytical Balance. The following rules embody the main points to be observed in the use and care of a balance.

(I) Each student must feel a personal responsibility for the proper use of his balance; the carelessness of any one may render inaccurate the work of all who use the same balance.

(2) Before use, with clean pans, the adjustment of the balance should be tested by the student.

The balance is properly adjusted only if the following conditions are fulfilled: (a) The spirit level or plumb bob inside the balance case should show that the balance is level; $(b)$ the mechanism for raising and lowering the beam should work smoothly; $(c)$ the pan arrests should just touch the pans when the beam is lowered; $(d)$ the pointer should rest at zero when the beam is raised; and also when it is lowered, with the pans supported; and $(e)$ the pointer should swing equal distances on either side of the zero when the beam is set in motion without any load on the pans. In the latter case, if the variation does not exceed two divisions on the scale, it is sufficient to allow for the small zero error, without adjustment.

(3) The beginner should not attempt to make adjustments himself, but should apply to the instructor in charge.

(4) In order to set the beam in motion, it should first be lowered so that the pans rest upon the pan arrests; the latter are then cautiously lowered, and, in case the beam fails to swing, it can be set in motion by means of the rider. There is, however, a "trick" in lowering the pan supports so that the oscillations of the pointer will have the required amplitude. ${ }^{1}$

The pans should be arrested and the beam raised before any change is made in the load or weights on the pans except in the

1 It is not necessary to employ very long swings; an amplitude of two scale divisions is probably sufficient, even in very exact work. See in this connection Horace L. Wells, Jour. Amer. Chem. Soc., vol. 42, p. 4 I I (I920). 
case of the small fractional weights, when it is only necessary to arrest the pans. The object to be weighed and the heavy weights should be placed in the middle of their respective pans, since a heavy load near the edge of a pan is apt to cause troublesome oscillations.

The beam and stirrups should never be left upon their knifeedges, and the motion of the beam should be arrested only by means of the pan arrests, and only when the pointer is passing the center of the scale; otherwise the knife-edges and their agate bearings are subjected to an unnecessary strain.

(5) The weights should be cared for as well as the balance. They should be handled carefully, and only with the forceps provided; they should never be touched with the fingers. In weighing, the weights should always be placed upon the same pan, and they should be taken in the order in which they occur in the box, the larger ones first; and the weight of the object should be recorded by noting the vacant spaces in the box. The record so obtained should be checked as the weights are removed from the pan. In this way errors are not likely to occur.

(6) Analytical samples should not be placed directly upon the balance pan, and the object to be weighed should not be warmer or colder than the air in the balance case. Currents of hot air will tend to buoy up one arm of the balance, and also to cause that arm to expand in length. ${ }^{1}$ If the object is colder than the atmosphere of the balance case, moisture may condense on its surface. If the body to be weighed is likely to be electrified (e.g. a glass weighing tube), it should be allowed to stand for some time after it has been wiped, before weighing.

(7) The balance case should be closed while weighing with the rider, so as to avoid currents of air.

As soon as the object is apparently balanced by the weights the beam should be raised and again lowered into place, and the

${ }^{1}$ For instance, a platinum crucible which appeared to weigh $20.649 \mathrm{~g}$. when warm, weighed $20.6920 \mathrm{~g}$. when cold. 
observation repeated. This insures the proper alignment of the beam and pans.

(8) In using weighing bottles or tubes, the vessel should be weighed together with its contents. The sample should then be removed without loss, and the vessel and contents again weighed. The difference in weight indicates the quantity taken. The weight of a tube, recorded at some previous time, should always be confirmed before weighing out a new sample from it.

(9) Errors in weighing should fall well within the limits of the experimental error due to the analytical operations. If, for example, an error of $0.00 \mathrm{I}$ g. were made in weighing out a gram sample of fireclay containing $0.25 \%$ of $\mathrm{MgO}$, the resulting error in the determination of the magnesia could be no greater than $0.1 \%$ of its value, which is negligibly small. A I mg. error, however, made in weighing the 0.0069 g. of $\mathrm{Mg}_{2} \mathrm{P}_{2} \mathrm{O}_{7}$ would involve an error of over $14 \%$ in the magnesia value, and this would be intolerable.

(Iо) Finally, if anything at all appears to be the matter with a balance, the instructor's attention should at once be called to the fact.

Determination of the Rest-point. The beam and stirrups are first lowered upon their knife-edges by slowly turning to the left the milled head at the front of the balance case; then the pans are released by gently pressing inwards the small button, also at the front of the case; and, with the beam swinging smoothly, a consecutive record is made of the number of scale divisions traversed by the pointer on either side of the center. The swings to the left are recorded as negative numbers and those to the right as positive numbers; in the determination of the rest-point, one more reading must be made on one side than on the other, and all of the readings must be consecutive. Upon dividing by 2 the algebraic sum of the averages for the two sides, the quotient is the rest-point of the balance for the case under consideration, i.e. the position on the scale at which the pointer would finally come to rest. 
Example:

\begin{tabular}{ll|lr|l}
\hline \hline \multicolumn{1}{c|}{ LEFT } & & \multicolumn{2}{|c|}{ RIGHT } & \\
\hline & -4.8 & & \\
& -4.6 & & \\
& -4.4 & & +2.5 & \\
\hline Average: & -4.6 & Average: & +2.6 & Rest-point $=-\mathbf{r . 0 .}$ \\
\hline
\end{tabular}

Two methods of procedure are now open to the operator. He may either make his weighings with reference to this observed rest-point or he may adjust the balance so that the observed rest-point is the actual zero of the scale. The first method is preferable, unless the rest-point is more in error than one scale division. The rest-point is apt to change, and it must be determined each day, or even more often.

Methods of Weighing. Weighings smaller than $0.005 \mathrm{~g}$. (or o.or g.) are made with the rider. When the arms are divided into five divisions, a 5-milligram rider is used; in general, the rider should weigh as many milligrams as there are large divisions between the central knife-edge and the right-hand stirrup support. Each large division on the beam then corresponds to a milligram.

Ordinary Method. The object to be weighed is placed upon the left-hand pan of the balance and weights upon the righthand pan, until, finally, the further addition of $5 \mathrm{mg}$. (or Io mg.) more than counterbalances the object. This weight is then removed, the balance case closed, and the rider adjusted so that the pointer swings equal distances on either side of the rest-point. This method of weighing is very common, and it is sufficiently accurate for ordinary analytical work. If necessary, the restpoint of the unloaded balance should be determined before each weighing.

In special cases, as in the calibration of a set of weights, it is important to make more accurate weighings. It is here best to 
employ the method of weighing by the use of deflections. Though this method may appear to be laborious, the labor is more apparent than real. The sensitiveness of the balance may be found by adding a small weight to one of the pans and noting how far the rest-point is deflected from its former position. Then, instead of adjusting small weights until the rest-point is brought to the proper place, we merely note the deflection from this point and calculate from the sensitiveness the weight that would be needed to bring the rest-point to the desired position. The sensitiveness will, in general, be different at different loads, and, especially with a very sensitive balance, it must be redetermined from time to time. For very accurate work, it is advisable to determine the sensitiveness at each weighing.

Method of Weighing by the Use of Deflections. (a) The restposition of the unloaded balance is determined, as already described. Let us suppose this to be at +o.I.

(b) The deflection of the rest-point per milligram, or the sensitiveness of the loaded balance, is determined. The object to be weighed is placed upon the left pan, the weights on the right pan. When the weights have been adjusted so that an additional $0.005 \mathrm{~g}$. (or $0.0 \mathrm{r}$ g.) would be too much (e.g. weights $=\mathrm{II} .2 \mathrm{I} 6 \mathrm{~g}$.), the balance case is closed, and the rider adjusted until the pointer swings on both sides of the zero of the scale. The rest-point is then found; for example, at to.8. The rider is moved one milligram division, in this case to the right, and the rest-point again determined; at, say, -2.I. That is, the rest-point is deflected $+0.8-(-2 . \mathrm{I})=2.9$ divisions by $\mathrm{I}$ milligram, in the case of that particular load.

(c) The weight of the object is calculated. In this case, the object weighs $11.216+x \mathrm{~g}$. The rest-point of this load is displaced $0.8-0.1=0.7$ scale division. Since 2.9 scale divisions correspond to I milligram, 0.7 scale division will correspond to $\frac{0.7}{2.9}=0.24 \mathrm{mg}$. Hence the weight of the body is 11.216+ 
$0.00024=11.21624 \mathrm{~g}$. These calculations may be summarized in the formula

$$
\text { Correction }=+\frac{a-z}{a-b} \mathrm{mg} \text {, }
$$

in which $z$ represents the rest-point of the unloaded balance, $a$, the rest-point with not quite enough weight on the right pan; and $b$, the rest-point with a milligram more on the right pan than corresponds to $a$.

Analytical balances will rarely indicate with certainty less than $0.000 \mathrm{I}$ g. Hence, although the weight may be calculated as above to the fifth decimal, it should generally be rounded off by dropping the fifth decimal and raising the fourth decimal one unit when the dropped figure exceeds 5 .

In certain cases, as in the calibration of volumetric measuring apparatus, it is necessary for the weight found to be independent of any inequality in length in the beam arms. In such cases, and in the determination of absolute weights (reduction to weights in vacuo), one of the following methods should be used.

Method of Weighing by Transposition. When the arms of a balance are nearly equal, the method of transposition furnishes a more accurate comparison than that of substitution (see below). It requires about the same amount of time for the observations, but the true weight is not shown in so direct a manner. Before and after the transposition, the rest-point of the loaded balance is noted and the added weight that would be needed to bring the rest-point to the desired position is determined as already described.

Having weighed the object first in one pan, then in the other, if we let $W$ be the true weight, $a$ the weights required to counterbalance the object when it is on the left pan, and $b$ the weights required when the object is on the right pan, then, according to the principle of moments :

That is, whence

$$
\begin{aligned}
W l & =a r ; \text { and } b l=W r \\
W^{2} l r & =a b l r, \text { or } W^{2}=a b \\
W & =\sqrt{a b} .
\end{aligned}
$$


Therefore the true weight is the square root of the product of the two observed weights.

Weighing by transposition is recommended for work of high precision in which it is also desirable to calculate the rest-point from several swings of the pointer. In other cases, substitution is generally to be preferred.

Method of Weighing by Substitution. Here the object, placed on the right-hand pan, is approximately balanced by a suitable tare (weights, wire, beaker containing shot, etc.) on the lefthand pan, and the rest-point determined. The object is then removed, and weights are added in its place until the rest-point is restored to its former position. These weights are necessarily the same in value as the object for which they substitute, irrespective of differences in the arms.

The Calibration of a Set of Weights. ${ }^{1}$ Fairly accurate weights can readily be purchased, and for most analytical work the inaccuracies of the better class of weights are negligibly small in comparison with the errors of experiment, and the imperfections in the analytical processes.

An analyst, however, should know that his weights are sufficiently accurate, and for this reason he should calibrate the weights. The errors in weighing due to imperfections in the weights can easily be reduced to $0.000 \mathrm{I} \mathrm{g}$. The weights should be tested at periodic intervals, say once or twice a year, depending upon the frequency with which they are used.

In special cases, e.g. in the calibration of volumetric apparatus, absolute weights may be required, but for general analytical work these are not necessary. If the weights are consistent with one another, their absolute values have no influence upon the accuracy of an analysis.

Duplicate and triplicate weights of a set should be so marked that they can be readily distinguished. A satisfactory method is to use one and two conspicuous dots on duplicates, and one,

1 In this connection, see Circular of the Bureau of Standards, No. 3, and also T. W. Richards, Jour. Amer. Chem. Soc., vol. 22, p. 144 (I900). 
two, and three such dots on triplicates, - stamped by means of a small punch. The designations of the weights being tested are conveniently inclosed in parentheses, to prevent their confusion with the numerical data for use in the calculations. And it is customary to express the results in terms of corrections to be applied in the use of the weights, rather than in terms of the total values; the plus sign for a correction indicating that the mass of a weight is greater than its nominal value, the minus sign that it is less. In using the weights, these signs are then treated in the ordinary algebraic manner. For example, if $(\mathrm{IO})=9.9987,\left(\mathrm{IO}^{\prime \prime}\right)=10.0008$, and $(5)=5.0002$, the respective corrections are $-\mathrm{I} .3$, +o.8, and +0.2 $\mathrm{mg}$.; the nominal value of the weights is $25.0000 \mathrm{~g}$., but their corrected value is $25.0000+$ (- I.3 +0.8+0.2) mg., or $24.9997 \mathrm{~g}$.

The weights are best calibrated according to their apparent masses as determined by comparison with brass standards in air; that is, in terms of "weight in air against brass." This method is advisable, owing to the prevailing use of small platinum and aluminum weights in connection with the larger weights of brass.

In the calibration of analytical weights, the rest-point should always be calculated from several swings of the pointer, and the individual calculations should be extended to five decimals. If the beam arms do not differ in length by more than $0.001 \%$, the simple method of deflections may be used; otherwise it is necessary to make use of transposition or substitution. The substitution method involves less work in calculating than that of transposition, but if it is used it is desirable to have a second set of weights to furnish the tares.

In the case of an ordinary analytical set of weights, from $20 \mathrm{~g}$. to $5 \mathrm{mg}$., the following comparisons should be made, with the use of the rider, as in ordinary weighings. 
A

The Small Weights ${ }^{1}$

(500) against $\mathbf{\Sigma}(500)$

(200) against $\left(\mathrm{roo}^{\prime}\right)+\left(100^{\prime \prime}\right)$

(1०o') against (roo")

(roo') against $\mathbf{\Sigma}($ roo)

(50) against $\boldsymbol{\Sigma}(50)$

(20) against $\left(\mathrm{ro}^{\prime}\right)+\left(\mathrm{ro}^{\prime \prime}\right)$

(ro') against (ro')

(ro') against $\Sigma(\mathrm{ro})$

(5) against (Rider at 5)

C

One-gram piece, $\left(\mathrm{I}^{\prime}\right)$, against $\mathbf{S}(\mathrm{r})$
$B$

The Larger Weights

(20) against $\left(\mathrm{IO}^{\prime}\right)+\left(\mathrm{IO}^{\prime \prime}\right)$

(Io') against (Iо')

(ro') against $\mathbf{\Sigma}$ (ro)

(5) against $\Sigma(5)$

(2) against $\left(\mathrm{r}^{\prime}\right)+\left(\mathrm{r}^{\prime \prime}\right)$

( $\left.\mathrm{I}^{\prime}\right)$ against $\left(\mathrm{r}^{\prime \prime}\right)$

$\left(\mathrm{x}^{\prime}\right)$ against $\left(\mathrm{r}^{\prime \prime \prime}\right)$

\section{$D$}

Ten-gram piece, $\left(\mathrm{ro}^{\prime}\right)$, against an absolute (known) standard of the same nominal value.

The weight of each of the smaller pieces is calculated from the data in terms of one of them, say the ten-milligram piece (IO'), as a standard; and, in the same way, that of each larger piece in terms of one of them, as the ten-gram piece $\left(\mathrm{I} \mathrm{O}^{\prime}\right)$. Then, in order to express all the weights of the set in terms of a common standard, the values of the fractional weights (in terms of their standard) are added, and the result compared with their total weight as found in terms of a one-gram piece, say $\left(\mathrm{r}^{\prime}\right)$.

Suppose, for example, the values of the small weights are: $(500)=499.03, \quad(200)=200.07, \quad(100)^{\prime}=100.10, \quad\left(1_{00}^{\prime \prime}\right)=100.00$, $(50)=49.87, \quad(20)=19.98, \quad\left(\mathrm{Io}^{\prime}\right)=10.00, \quad\left(\mathrm{Io}^{\prime \prime}\right)=\mathrm{10.03}, \quad(5)=$ 5.00 , and (Rider at 5$)=5.00-i . e . \Sigma(\mathrm{r})=0.99908$; while, in terms of the one-gram piece $\left(I^{\prime}\right)$, their collective weight is 1.00165 . Then, in order to express the values in terms of $\left(\mathrm{I}^{\prime}\right)$, each of them should be multiplied by $\frac{\mathrm{x} .00 \mathrm{I} 65}{0.99908}=\mathrm{I} .00257$. If, however, in terms of the ten-gram piece $\left(\mathrm{I} \mathrm{O}^{\prime}\right),\left(\mathrm{I}^{\prime}\right)=0.99954$, the provisional

1 The designations $\Sigma(500)$, etc., are here used to represent collective weights of the nominal values indicated; in this instance, $(200)+\left(100^{\prime}\right)+\left(100^{\prime \prime}\right)+(50)+(20)$ $+\left(\mathrm{Io}^{\prime}\right)+\left(\mathrm{Io}^{\prime \prime}\right)+(5)+($ Rider at 5$)$. 
value of each fractional piece may be multiplied directly by $\frac{\mathrm{r} .00 \mathrm{I} 65 \times 0.99954}{0.99908}=\mathrm{I} .002 \mathrm{II}$, to express their weights in terms of that of the ten-gram piece ( $\left.\mathrm{IO}^{\prime}\right)$.

What we actually do, assuming ( $\left(\mathrm{Io}^{\prime}\right)$ is found to have an abso= lute value of $9.99970 \mathrm{~g}$., is to multiply the individual values of the larger pieces, based upon (ro') as a standard, by 0.99997 , and those of the fractional weights, expressed in terms of the tenmilligram piece $(\mathrm{IO} \prime)$, by $\frac{\mathrm{I} .00 \mathrm{I} 65 \times 0.99954}{0.99908} \times 0.99997=\mathrm{I} .00208$. In this way, we finally arrive at the individual values of the pieces, both large and small, in terms of the absolute standard.

Errors Due to Inequalities in Length in the Beam Arms. In the preceding discussion, it has mainly been assumed that the two arms of the beam are equal in length. This is not really the case. It is mechanically impossible to insure perfect equality. To find the relative lengths of the arms, place (corrected) weights of the same nominal value - say, 50 grams - upon each pan, and bring the balance into equilibrium by means of the rider. Interchange the weights on the two pans, and again bring the balance into equilibrium by means of the rider. Call the two weights $W$ and $w$, and let $l$ and $r$ respectively denote the additional weights required for equilibrium on the left and right sides. Then, on the first weighing, $w+l=W$; and, on the second weighing, $W=w+r$. Let $L$ and $R$ respectively denote the length of the left and right arm. Then from the law of levers,

$$
L(w+l)=R W \text {; and } L W=R(w+r) .
$$

Solving each of these equations for $W$, and equating the results, we find that

$$
\frac{L}{R}(w+l)=\frac{R}{L}(w+r)
$$

whence,

$$
\frac{L}{R}=\sqrt{\frac{w+r}{w+l}}
$$


Suppose, for example, with use of true weights, that the weighings were found to be:

$$
\begin{aligned}
& \text { LEFT RIGHT } \\
& \text { (50) } \quad=(20)+\left(\mathrm{IO}^{\prime}\right)+\left(\mathrm{IO}^{\prime \prime}\right)+\left(\mathrm{IO}^{\prime \prime \prime}\right)+0.13 \mathrm{mg} \text {. } \\
& (20)+\left(\mathrm{Io}^{\prime}\right)+\left(\mathrm{Io}^{\prime \prime}\right)+\left(\mathrm{Io}^{\prime \prime \prime}\right)=(50)+0.19 \mathrm{mg} \text {. }
\end{aligned}
$$

Here, then, $l=-0.000 \mathrm{I}_{3}, r=+0.000 \mathrm{I} 9$, and $w=50 \mathrm{~g}$. Consequently if in the above expression we let $R=\mathrm{I}$, we have

$$
L=\sqrt{\frac{w+r}{w+l}}=\mathrm{I} .0000032
$$

i.e.

$$
L: R=\mathrm{r} .0000032: \mathrm{I}
$$

With this ratio, $\frac{L}{R}=1.0000032$, a weight $w$ on the left pan of the balance will be equivalent to a weight $w \times 1.0000032$ on the right pan. Hence, if an object on the left pan balances the weight $50 \mathrm{~g}$. on the right pan, the weight of the object is $50.0000 \times 1.0000032=50.0016 \mathrm{~g}$. - an error of only 0.003 per cent. There is therefore no need to apply the correction. Each balance has its own constant $L: R$ for a given load; the numerical value of the ratio varies with the different loads.

Most analytical balances do not require a correction on account of inequalities in the arms; the lengths are usually sufficiently exact. Anyhow, if the weights are always placed, say, on the right pan, such a correction is unnecessary in ordinary analytical work, because the weights observed are proportional to the true weights, and the ratios obtained are not affected.

Errors Due to Atmospheric Buoyancy. Two bodies equal in weight at the same time and place contain the same mass or quantity of matter only if the weighing is carried out in a vacuum, or if the bodies have the same volume. The latter is hardly ever the case.

A body weighed in air is buoyed up by a pressure equivalent to the weight of the air which it displaces. Suppose that exactly Io grams of platinum (sp. gr., 21.55) are weighed in air with 
brass weights (sp. gr., 8.4). Then $0.45 \mathrm{cc}$. of air, at say $20^{\circ}$ and $760 \mathrm{~mm}$., i.e. about $0.00054 \mathrm{~g}$., are displaced by the platinum; while the weight of air displaced by Io grams of brass is 0.00143 g. Hence, the weight of brass which will be required to counterpoise 10 grams of platinum is 10 $+(0.00143-0.00054)=10.0009 \mathrm{~g}$.

The arithmetic of the above calculation is summarized in the formula :

$$
\text { Corrected weight }=w+\omega\left(\frac{w}{s}-\frac{w}{s_{1}}\right)
$$

in which $w$ represents the apparent weight of the object; $s$, the specific gravity of the object; $s_{1}$, the specific gravity of the weights; and $\omega$, the weight of a cubic centimeter of air under the conditions prevailing at the time of the experiment.

To illustrate the effect of the buoyancy of air on a few common substances, when weighed with brass weights, it may be stated that the error per gram of substance weighed is $0.1 \mathrm{mg}$. for ferric oxide (sp. gr. 5.I 2); 0.3 mg. for $\mathrm{Mg}_{2} \mathrm{P}_{2} \mathrm{O}_{7}$ (sp. gr. 2.40); and $0.4 \mathrm{mg}$. for sodium chloride (sp. gr. 2.13).

In ordinary analytical operations we have to deal with differences in weight, and with ratios, not with absolute weights. When the amount of a precipitate is determined from the difference in the weight of an empty crucible and of the crucible plus the precipitate, the buoyancy correction is not needed for precipitates with a specific gravity near that of the substance undergoing analysis. If, however, the specific gravities are widely separated, it may be worth while to correct for buoyancy. For instance, since the specific gravities of iron ore and barium sulphate are nearly equal, it would be a waste of time to correct for buoyancy in determining sulphur in an ore of iron. On the other hand, in standardizing a solution of silver nitrate by precipitating silver chloride from a specific weight of the solution (use of weight burettes), the buoyancy of air may cause an error of $0.1 \%$.

Summary. The foregoing discussion clearly illustrates the advisability, in work of an accurate nature, of always estimating 
the effect of the various sources of error on the final result. Any of these errors can then be neglected, provided it is sufficiently small in comparison with the error derived from other sources. The chief sources of error involved in weighing are those due to: (I) variations in the rest-point of the balance; (2) inconsistent weights; (3) inequalities in length in the beam arms; and (4) atmospheric buoyancy. In weighings making any pretense to accuracy, the following points should be noted:

(I) The rest-point of the unloaded balance should be determined and made use of in each weighing.

(2) The weights should be calibrated, and periodically checked for consistency.

(3) The errors due to inequality in length in the beam arms may be neglected in ordinary analytical work.

(4) Although the correction of weighings for buoyancy can almost always be neglected in general analytical work, owing to the much larger errors associated with the preparation of precipitates for weighing, it is advisable in weighing bulky glass apparatus (potash bulbs, etc.), to use a similar piece of apparatus as a counterpoise. This will serve to eliminate any errors due to variations in temperature, pressure, and humidity during the course of the determination.

\section{PRECIPITATION}

Qualities Desirable in Precipitates Which Are to be Used in Gravimetric Determinations. Precipitation is made use of more often than any other means for the separation of inorganic substances. But, in carrying out such separations, precipitates should conform as nearly as possible to the following ideal specifications: (I) The precipitate should be insoluble in the mother liquid, and also in the wash liquid to be used; (2) it should be compact, easy to filter and wash; (3) it should be a pure, non-volatile chemical substance of known percentage composition, or it should yield upon ignition a pure, non-volatile substance of known composition. The last condition is of 
course of especial importance, if the precipitate is the substance finally to be weighed. Moreover, other things being equal, it is conducive to accuracy if a precipitate can be obtained which contains a low percentage of the substance under investigation (cf. Part V, Problem 46).

But few processes satisfy all these requirements, and in the case of any analytical process it is important to know what conditions favor and what conditions hinder the separation and purification of a given precipitate. There are a few general principles of such wide applicability that they should be constantly borne in mind. Their discussion follows.

Colloidal and Fine-grained Precipitates. Finely divided precipitates, such as newly precipitated silver chloride, barium sulphate, calcium oxalate, etc., are particularly liable to pass through the filter; furthermore, they tend in large measure to stop up the pores of the filter, and thus to increase the time required for filtration and washing. Hence, the analyst employs various artifices in order to enlarge the size of the particles.

(I) The grain size can frequently be increased by allowing the fine grains which originally separate to digest in the precipitation liquid. This change is more rapid in the hot, than in the cold, mother liquid. In the case of crystalline substances, it often happens that the finer grains, which (owing to differences in surface tension) are somewhat more soluble than the coarser ones, redissolve; and since the solution is then supersaturated in respect to the coarser grains, these are augmented in size by the surface deposition of the material furnished by the finer grains.

The boiling of liquids containing colloidal substances frequently leads to the flocculation of a large number of fine particles into a smaller number of coarser aggregates.

(2) Precipitates produced in hot solutions are often coarsergrained than if produced in cold solutions. From what has just been said, the reasons for this fact will be apparent. 
(3) The flocculation of a precipitate which separates in a colloidal condition is frequently caused by the salts present in the mother liquid. When these salts have been almost removed, during the washing, the colloidal precipitate is apt to be deflocculated, and it may then give a turbid filtrate, or become so slimy as to be almost impermeable to the wash liquid. In such cases it is necessary to wash the precipitate either with boiling water, or, better, with the solution of an electrolyte which will prevent the deflocculation of the precipitate, and which can be easily removed by drying or ignition. Sometimes dilute acids can be used, but usually, for obvious reasons, we have to depend upon volatile ammonium salts.

The Contamination of Precipitates. Finally, it siould be noted that the finer the grain of the precipitate, the greater will be the quantity of contaminating salts likely to be retained by the wet precipitate. The salts appear to be retained by a kind of surface attraction, called adsorption, ${ }^{1}$ and, since fine-grained precipitates expose a larger surface of separation between the solid and the liquid phases, and also because of their compactness, the fine grained precipitates are more difficult to wash clean than those of coarser texture. Colloidal gelatinous precipitates like ferric and aluminum hydroxides are in an extremely fine state of subdivision, and, in consequence, they are most difficult to wash clean.

In addition to their tendency to be contaminated by the adsorption of salts, precipitates are also frequently liable to contamination, owing to the formation during precipitation of more or less stable insoluble complexes (and, in rare cases, possibly, to the carrying down of foreign substances by the precipitate in a state of solid solution). These impurities, in whatever form they may be present, cannot be completely removed by washing, and the wash water will frequently fail to

1 But see "The Contamination of Precipitates in Gravimetric Analysis," G. McP. Smith: Journal of the American Chemical Society, vol. 39, pp. 1152-73 (I9I7). 
show any indication of the impurities which are still present in the precipitate.

It is therefore often advisable to redissolve the precipitate, and to repeat the precipitation. The objectionable impurity divides itself in a more or less definite concentration ratio between the precipitate and the mother liquid. A relatively large amount may be retained by the precipitate in the first precipitation, but in a second precipitation, when only that amount of salt retained by the first precipitate is in solution, the division of the undesirable substance between the precipitate and the solution in the given concentration ratio means that a much smaller quantity of impurity will be retained by the second precipitate. Repeated precipitations will, in general, soon carry the amount of impurity outside the range of the balance; but, in carrying out such operations, the solubility relations of the precipitate itself should never be left out of consideration.

The Theory of Precipitation. Reversible Reactions. The reactions which are made use of in analytical chemistry belong mostly to the reversible type. Under unfavorable analytical conditions, instead of running to completion, such reactions may come to an apparent standstill, owing to a too early attainment of equilibrium, and in that case a certain quantity of the substance involved in the determination is likely to remain unaccounted for. This is true in many reactions involving precipitation, oxidation and reduction, and even neutralization. Furthermore, many reactions are more or less influenced by the presence of certain substances. It is obvious that a knowledge of the processes which take place in such cases will be of the greatest service to the analytical chemist.

Therefore, it is of the first importance in analytical chemistry to study each process thoroughly in detail, in order to find out and understand the conditions which will most nearly lead to the completion of every reaction involved. For such studies, the ionic theory and the law of mass action are indispensable guides. It is taken for granted that, at this poin', the student is already 
sufficiently familiar with the qualitative conception of ionization.

Degree of Ionization. In a dilute aqueous salt solution, the greater, ${ }^{1}$ and by far the most active, portion of the salt is almost invariably ionic. But with acids and bases there is a wider range, and of these a larger number are less highly ionized; but even here the ions are nearly always much more active than the non-ionized molecules. The acids and bases that are commonly called "strong" are highly ionized, i.e. their solutions are especially active as acids or bases because they contain high hydrogen, or hydroxide-ion, concentrations.

Composition of the Ions. . It is usual to assume the simplest possible compositions for the ions formed upon the dissociation of any given electrolyte. A more careful study of the subject, however, shows that the ionization of even simple electrolytes may be a very complicated process. It is known, for example, that sulphuric acid contains ions of the formula $\mathrm{HSO}_{4}^{-}$, in addition to $\mathrm{SO}_{4}^{--}$ions, and that phosphoric acid yields ions of the formulas $\mathrm{H}_{2} \mathrm{PO}_{4}^{-}, \mathrm{HPO}_{4}^{--}$, and $\mathrm{PO}_{4}^{---}$. All of these are probably more or less highly hydrated; even hydrogen and hydroxide ions are supposed to be hydrated in aqueous solution. Further, many metallic ions show a decided tendency to exist in combination with certain molecules and radicals, as $\mathrm{OH}_{2}$, $\mathrm{OH}, \mathrm{NH}_{3}, \mathrm{NH}_{2}, \mathrm{CN}, \mathrm{C}_{2} \mathrm{O}_{4}, \mathrm{PO}_{4}, \mathrm{Cl}$, etc. ; but in very dilute solutions these complexes are apt to be more or less highly dissociated into their constituents.

The Law of Mass Action as Applied to Ionic Equilibria. In aqueous solution, acetic acid is supposed to ionize as follows:

$$
\mathrm{HC}_{2} \mathrm{H}_{3} \mathrm{O}_{2} \rightleftarrows \mathrm{H}^{+}+\mathrm{C}_{2} \mathrm{H}_{3} \mathrm{O}_{2}^{-} \text {. }
$$

The quantity of the molecular acid that is ionized per unit of time in a given volume of the solution is proportional to the

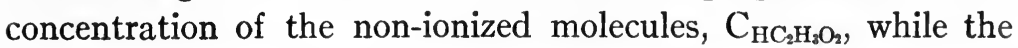

1 Noteworthy exceptions are mercuric chloride and cyanide, lead acetate, and a few others. 
quantity of the molecular acid that is simultaneously formed by the union of the ions depends upon the frequency of the encounters of the two kinds of ions, which in turn is proportional to the product of their concentrations, $\mathrm{C}_{\mathrm{H}^{+}} \mathrm{X}_{\mathrm{C}_{2} \mathrm{H}_{3} \mathrm{O}_{2}}-$.

The speeds of the respective actions will therefore be

$$
S_{1}=\mathrm{C}_{\mathrm{HC}_{2} \mathrm{H}_{3} \mathrm{O}_{2}} \times F_{1} \text { and } S_{2}=\mathrm{C}_{\mathrm{H}^{+}} \times \mathrm{C}_{\mathrm{C}_{2} \mathrm{H}_{3} \mathrm{O}_{2}}-\times F_{2} \text {, }
$$

in which $F_{1}$ represents the intrinsic tendency of $\mathrm{HC}_{2} \mathrm{H}_{3} \mathrm{O}_{2}$ to ionize, and $F_{2}$ that of $\mathrm{H}^{+}$and $\mathrm{C}_{2} \mathrm{H}_{3} \mathrm{O}_{2}-$ to combine.

When equal amounts of material are being transformed each way, i.e. at equilibrium, $S_{1}=S_{2}$, and therefore

or

$$
\begin{aligned}
& \mathrm{C}_{\mathrm{HC}_{2} \mathrm{H}_{3} \mathrm{O}_{2}} \times F_{1}=\mathrm{C}_{\mathrm{H}^{+}} \times \mathrm{C}_{\mathrm{C}_{2} \mathrm{H}_{3} \mathrm{O}_{3}}-\times F_{2}, \\
& \frac{\mathrm{C}_{\mathrm{H}^{+}} \times \mathrm{C}_{\mathrm{C}_{2} \mathrm{H}_{3} \mathrm{O}_{2}^{-}}}{\mathrm{C}_{\mathrm{HC}_{2} \mathrm{H}_{3} \mathrm{O}_{2}}}=\frac{F_{1}}{F_{2}}=k
\end{aligned}
$$

$\frac{F_{1}}{F_{2}}$, being the ratio of two constants, is constant; and the value, $k$, of this ratio of the affinities driving the opposed actions is called the affinity constant of the reversible reaction. At any given temperature, provided the solution is dilute, the numerical value of $k$ remains the same no matter what the total concentration of the solution may be. ${ }^{1}$ In the case of acetic acid, for

\begin{tabular}{|c|c|c|c|}
\hline $\begin{array}{l}\text { TOTAL Molal } \\
\text { CONCENTRATION } \\
\text { OF ACID }\end{array}$ & $\begin{array}{l}\text { PROPORTION } \\
\text { IONIZED }\end{array}$ & $\begin{array}{c}\text { MOLAL CONCENTRATION } \\
\text { OF H+AND OP AC- } \\
\left(\mathrm{C}_{\mathrm{H}}+\mathrm{AND} \mathrm{C}_{2} \mathrm{H}_{3} \mathrm{O}_{2}-\text { ) }\right.\end{array}$ & $\begin{array}{c}\text { Molal ConCENTRATION } \\
\text { OP HAC } \\
\left(\mathrm{CHC}_{33 \mathrm{O}}\right)\end{array}$ \\
\hline 1.000 & 0.0041 & $0.004 \mathrm{I}$ & $1.000-0.004 \mathrm{r}$ \\
\hline 0.1000 & 0.0130 & 0.00130 & $0.1000-0.00130$ \\
\hline 0.0100 & 0.0407 & 0.000407 & $0.01000-0.000407$ \\
\hline
\end{tabular}
example, the following figures have been obtained, at $\mathrm{I} 8^{\circ}$, from conductivity determinations.

${ }^{1}$ When data such as the following are applied to cases of soluble, highly ionized substances, the $k$-values so obtained for any given compound are usually far from constant. The general conclusions arrived at through the application of such data are, however, as a rule, not invalidated by this fact. 
Substituting these figures in equation (I) above, we get:

$$
\frac{(0.004 \mathrm{I})^{2}}{0.996}=0.0000169 ; \frac{(0.0013)^{2}}{0.0987}=0.000017 \mathrm{r} ;
$$

and

$$
\frac{(0.000407)^{2}}{0.00959}=0.0000172 \text {. }
$$

It is seen that, although the third solution is a hundred times more dilute than the first, and although the degree of ionization has increased tenfold, the value of $k$ is the same in both cases.

The Common-Ion Effect. When, through the presence of two substances which furnish an ion in common, the concentrations of the positive and negative ions of an ionogen are unequal, the law of mass action still holds.

Let us imagine, for example, that, by mixing equal volumes of the double-molal solutions, a solution is obtained which is uni-molal in respect to acetic acid and also to sodium acetate. Let us further suppose that the equilibria which exist in the mixture have been established in two separate stages, as follows: (I) that the concentrations of each undissociated compound and its ions have changed from those which exist in a doublemolal, to those which exist in a uni-molal solution of that compound; and (2) that the concentrations of all the substances present have changed from those which exist in the separate uni-molal solutions of the compounds, to those which exist in the mixture which is uni-molal in respect to each compound. Let us now consider this latter stage in detail.

In uni-molal solution, sodium acetate is 0.53 ionized, while acetic acid at that concentration is only 0.004 ionized. Each compound furnishes acetate ions, and the acetate ions present are all available, either for union with sodium ions, or for union with hydrogen ions. Initially, therefore, in the case of the sodium acetate, we have $\frac{0.53 \times 0.534}{0.47}>k_{1}$, instead of $\frac{0.53 \times 0.53}{0.47}=k_{1}$; but the two expressions are so nearly identical that we see at a glance that the ionic equilibrium of the salt will not be 
affected appreciably by the presence of the acid. In the case of the acetic acid, however, we have the initial relationship, $\frac{0.004 \times 0.534}{0.996}=\mathrm{I} 33 k$, instead of $\frac{0.004 \times 0.004}{0.996}=k$. Since, at equilibrium, the fraction $\frac{\mathrm{C}_{\mathrm{H}^{+}} \times \mathrm{C}_{\mathrm{C}_{2} \mathrm{H}_{3} \mathrm{O}_{2}^{-}}}{\mathrm{C}_{\mathrm{HC}_{2} \mathrm{H}_{3} \mathrm{O}_{2}}}$ remains constant, and since, owing to the low $\mathrm{H}^{+}$-ion concentration, $\mathrm{C}_{\mathrm{HC}_{2} \mathrm{H}_{3} \mathrm{O}_{2}}$, cannot be increased appreciably, nor $\mathrm{C}_{\mathrm{C}_{2} \mathrm{H}_{2} \mathrm{O}_{2}-}$ be appreciably diminished, by the formation of the molecular acid, it follows that the value of $\mathrm{C}_{\mathrm{H}^{+}}$must be lowered to about $\frac{1}{130}$ of its initial magnitude. That is to say, the sodium acetate in this solution diminishes the hydrogen-ion concentration from 0.004 to about 0.00003 .

The student should especially note that the concentration of a given ion can be lowered in this way to a value approximating zero only when that ion unites with an ion added to form a substance which is insoluble or which by nature has only a very slight tendency to dissociate. We might add sodium chloride in the hope of repressing the ionization of hydrochloric acid; but, since both compounds ionize highly, we should obtain no appreciable effect. If, however, we add sodium acetate in excess to hydrochloric acid, we can obtain a solution which is as weakly acid as the one discussed above. ${ }^{1}$

The Solubility Product. One of the commonest and most interesting applications of the law of mass action is met with in connection with the precipitation and solution of relatively insoluble salts.

Every substance possesses, when immersed in a liquid, a certain solution-tension, by which is meant an expansive force which tends to drive particles of the substance outward into the liquid. These particles move in every direction, and consequently some of them return to the solid and reattach them-

${ }^{1}$ For example, I mol of $\mathrm{HCl}+2$ mols of $\mathrm{NaC}_{2} \mathrm{H}_{3} \mathrm{O}_{2}$, in a volume of $\mathrm{I}$ liter, give a solution which is uni-molal in respect to acetic acid, to sodium acetate, and to sodium chloride. The hydrogen-ion concentration of this solution would also approximate 0.00003 . 
selves to it. This occurs the more and more frequently, as the concentration of the particles in the liquid increases, until, finally, a stage is reached at which the number of particles leaving the solid per unit of time is equal to the number deposited upon it. When the entire liquid is equally charged with dissolved particles, the liquid immediately surrounding the solid will lose none by diffusion, and a condition of equilibrium will be established. At a constant temperature, the quantity of dissolved solute will remain thereafter unchanged, no matter how long the materials are left in contact. It is at this point that the solution is said to be saturated with the dissolved substance.

In the case of silver bromate in water, we have the following scheme of equilibria :

$$
\begin{aligned}
& \mathrm{AgBrO}_{3} \rightleftarrows \mathrm{AgBrO}_{3} \rightleftarrows \mathrm{Ag}^{+}+\mathrm{BrO}_{3} \text { - } \\
& \text { (solid) (dissolved) }
\end{aligned}
$$

The solid $\mathrm{AgBrO}_{3}$ molecules tend to enter the solution, while at the same time dissolved $\mathrm{AgBrO}_{3}$ molecules tend to come out of solution, and the solution is saturated when these tendencies produce equal effects. The ions themselves (and any foreign materials present) are not supposed to take any direct part in the equilibrium which controls solubility. That is, in solutions saturated at a given temperature by a given solute, the concentration of the non-ionized molecules will be constant no matter what other substances may be present, provided that the quantities of all the dissolved substances are not sufficient to alter the nature of the solvent.

The total solubility of an ionogen, as we ordinarily use the term, is made up of a molecular and an ionic part. The quantity of the latter does not remain constant when a foreign substance giving a common ion is added to the solution. In a solution of silver bromate, for example, we have the mathematical relationship:

$$
\frac{\left(\mathrm{Ag}^{+}\right) \times\left(\mathrm{BrO}_{3}^{-}\right)}{\left(\mathrm{AgBrO}_{3}\right)}=k \text {, or }\left(\mathrm{Ag}^{+}\right) \times\left(\mathrm{BrO}_{3}^{-}\right)=k \times\left(\mathrm{AgBrO}_{3}\right) \text {. }
$$

But, since, in the special case of a solution which is saturated with the salt at a given temperature, the concentration of the non- 
ionized molecules, $\left(\mathrm{AgBrO}_{3}\right)$, remains constant, it follows that the product, $k \times\left(\mathrm{AgBrO}_{3}\right)$, also remains constant, or that in a saturated solution of a given slightly soluble ionogen the product of the concentrations of its ions is constant. This product is called the solubility product, because the two separate values jointly determine the magnitude of the total solubility of the ionogen. The concentration of the non-ionized molecules cannot be diminished, but the ionic part of the solute may become vanishingly small if the concentration of the common ion is made great as compared with that of the other ion of the solute. The relationships which exist in the case of silver bromate are illustrated in the following table, where it will be seen that, in this instance, the experimental values agree remarkably well with the calculated ones.

Solubility of $\mathrm{AgBrO}_{3}$ in Mols per Liter

\begin{tabular}{|c|c|c|c|}
\hline \multirow{2}{*}{$\begin{array}{c}\text { MOLS PER LITER OF } \\
\text { COMMON-ION SALT } \\
\text { ADDED }\end{array}$} & \multicolumn{2}{|c|}{ SOLUBILITy FoUND } & \multirow{2}{*}{$\begin{array}{c}\text { SolubiLIty CaLC. } \\
\begin{array}{c}\text { (Addition of either } \\
\text { Salt) }\end{array}\end{array}$} \\
\hline & $\begin{array}{c}\text { Addition of Silver } \\
\text { Nitrate }\end{array}$ & $\begin{array}{c}\text { Addition of Potassium } \\
\text { Bromate }\end{array}$ & \\
\hline 0 & 0.00810 & 0.00810 & $\longrightarrow$ \\
\hline 0.00850 & 0.00510 & 0.00519 & 0.00504 \\
\hline 0.0346 & 0.00216 & 0.00227 & 0.00206 \\
\hline
\end{tabular}

The theory of the precipitation and solution of slightly soluble ionogens may be summed up as follows : ${ }^{1}$

1 That is, of uni-univalent ionogens. In other cases, the solubility product would often contain ion-concentrations raised to the second, third, etc., powers; but in reality the question is very much complicated by interfering reactions. Thus, in the case of $\mathrm{PbCl}_{2}$, if $\mathrm{NaCl}$ is added to the saturated solution, some $\mathrm{PbCl}_{2}$ will be precipitated in accordance with the theory; but the addition of $\mathrm{Pb}\left(\mathrm{NO}_{3}\right)_{2}$ actually increases the solubility of the $\mathrm{PbCl}_{2}$. This is probably because of the formation of complexes, or of intermediate ions, such as $\mathrm{PbCl}+$, or of both, whereby the addition of the salt giving the common bivalent ion may not only fail to increase the concentration of the bivalent ion, but may even lower that of the univalent ion. At any rate, enough is known to indicate that the theory may not be so much at fault as we ourselves, in our lack of methods for finding out just what ions and complexes are present in such solutions, and in what quantities. 
Whenever the ion-concentration product of any pair of ions in a solution is made to exceed in value the solubility product of the compound formed by their union, the latter will be precipitated until the ion-concentration product has been reduced to the solubilityproduct value. And conversely, whenever the ion-concentration product of any pair of ions is made less than the solubility-product value, the compound formed by their union will be dissolved by the solution, if furnished in excess, until the solubility-product value has been regained.

\section{FILTRATION AND THE WASHING OF PRECIPITATES}

The purpose of filtration is to separate a solid from a liquid in which it is suspended. This is effected by causing the liquid to pass through a porous medium compact enough to retain the solid. The most important media in use are filter paper, asbestos pulp, and platinum sponge.

The Selection and Use of Paper Filters. Three qualities which are desirable in a filter are: (I) porosity, to insure rapid filtration; (2) sufficient compactness, to insure complete retention of the precipitate; and (3) low amount of ash. Filters for quantitative work, readily obtainable on the market, are such as have been treated with hydrochloric and hydrofluoric acids. Upon incineration, they leave a small, definitely known weight of ash. ${ }^{1}$

Rapid (porous) filters should be used for all precipitates which do not pass through them, and slow (compact) papers should not be used unless necessary. A great amount of time is consumed, often wasted, in the filtration and washing of precipitates.

The size of the filter should be determined, not by the volume of liquid to be filtered, but by that of the precipitate; the latter should not more than half fill the paper, but too large a paper leads to a waste of time in washing. The filter, as well as the precipitate, retains certain salts very tenaciously.

${ }^{1}$ For example each $9 \mathrm{~cm}$. filter of a certain well-known brand leaves on ignition an ash weighing about $0.1 \mathrm{Img}$. 
Funnels should be provided with narrow stems of even bore, about eight inches long, and should have an angle of $60^{\circ}$, so that a twice folded filter when opened up will closely fit its walls; the top edge of the filter should always be well below the rim of the funnel.

Upon being placed in the funnel, the paper should be wetted, and carefully bedded against the funnel walls, so that, when water is poured in, the stem of the funnel will be filled, without the entrance of air. ${ }^{1}$ If properly bedded in a clean funnel, water will rapidly pass through, and the paper will be at its best for service as a filter; the filtration proceeds under a pressure equivalent to the weight of a column of water of the same diameter as the bore of the stem and of a height equal to the length of the stem plus the depth of liquid in the filter.

If suction is to be applied, the paper should preferably be compact, and its apex should be supported by a perforated cone of platinum (or of palau), placed beneath the filter in the funnel. In general, if accuracy is aimed at, one should hesitate to use suction with paper filters.

After the precipitate has been allowed to settle, the clear liquid should be transferred to the filter by means of a glass rod, along which it should be made to flow towards the side, not the center of the paper. The outlet of the funnel should rest against the inner side of the receiving vessel, to enable the liquid to run down quietly, without danger of loss by splashing.

Before beginning to wash a precipitate, the receiving vessel for the filtrate should always be replaced by a clean beaker; although the precipitate may have shown no tendency to pass through the filter, it is often possible that it may be taken into colloidal solution by the wash liquid.

In general, the precipitate can be most efficiently washed by decantation, i.e. by the addition of successive portions of wash

1 If this fails to take place, either the stem of the funnel is too wide, or it is not free from grease. The latter can be removed by means of warm cleaning solution. 
liquid, followed by settling and the transfer of the clear liquid to the filter. Finally the precipitate itself may be transferred, and the washing completed upon the filter.

It will always be found that small portions of the precipitate adhere to the walls and bottom of the containing vessel. These can be rubbed loose by means of a so-called "policeman," - a piece of soft rubber tubing tightly fitted on the end of a glass rod. Pieces of rubber tubing with closed ends are sold for the purpose. These rubber-tipped rods should be used only for the above-mentioned purpose; they should never be used as stirring rods or be allowed to stand in analytical solutions.

The washing of precipitates should always be completed promptly after filtration; if allowed to stand, they are apt to dry out and crack, with the formation of channels for the free passage of wash liquid.

After filtering off a precipitate, the filtrate should always be tested to insure complete precipitation, and, after several washings have been made, the last washings should be tested; only a few drops of the latter should be taken at first if the filtrate contains substances to be subsequently determined, but near the end of the washing several cubic centimeters should be used. The necessity of making these tests cannot be too strongly impressed upon the student; no exception should be made.

Wash Bottles. Wash bottles for use in quantitative work usually consist of rubber-stoppered flasks of 250-500 cc. capacity, of resistance glass, with tubes smoothly bent, and with a jet made flexible through a joint with the outlet tube, by means of a short piece of black rubber tubing. The jet should deliver a thin even stream of liquid. For use with hot water, the neck of the flask may be wrapped with asbestos twine, or other material.

Gooch's Filtration Crucible. In I878, F. A. Gooch proposed, for the separation of certain precipitates by filtration with suction, the use of a mat of asbestos bedded on the perforated bottom of a crucible, thus rendering it possible to wash, dry, and weigh the precipitate in the crucible. 
Preparation of the Asbestos. Of the several varieties of asbestos on the market, the long-fiber, silky, chrysolite asbestos is best suited for this purpose. The asbestos should be rubbed roughly over the surface of a ro-mesh brass sieve, inverted on a piece of paper, until a sufficient amount has passed through. This is shaken up with water, allowed for the most part to settle, and the very fine particles poured off with the water. The pulp is then digested for one hour on the steam bath with concentrated hydrochloric acid, in a covered porcelain dish. At the end of this operation, water is added, and the liquid is poured off through a funnel provided with a platinum filtration cone; the asbestos being washed with hot water, at first by decantation, and finally in the funnel, with gentle suction, until the washings give no opalescence with silver nitrate solution. The washed asbestos is finally mixed with distilled water, and preserved in a glassstoppered bottle.

Preparation of the Gooch Filter. The Gooch crucible is connected with the suction apparatus by means of an adapter a glass cylinder $5^{-6} \mathrm{~cm}$. long, of about $3 \mathrm{~cm}$. inside diameter, which is drawn out at the bottom into a tube of about the same length, for passage through the rubber stopper of the suction flask. A $4-\mathrm{cm}$. length of band tubing is stretched over the mouth of the adapter, so that about half of it projects above the top, and the crucible is pressed into this projecting end of the rubber tube.

With the connections all well made, suction is gently applied, and asbestos suspension is gradually added until a smooth mat, not over $1.5 \mathrm{~mm}$. in thickness, is formed; this is then covered with a thin perforated porcelain disk (filter plate), and enough asbestos added to barely cover the disk. With increased suction, water is next filtered through the crucible until, when held before a bright light, no trace of suspended asbsetos can be seen in the washings. Usually, 250-500 cc. of water will suffice to render the mat stable upon further washing. The filter plate is used to protect the asbestos mat during filtration 
and washing; if the perforations of the crucible are rather large, it is advisable also to use a filter plate beneath the mat.

After the preparation of a satisfactory mat, the crucible, placed in a little beaker, is dried for an hour at $120-130^{\circ}$, in an oven; it is then allowed to cool in a desiccator, and weighed. This process is repeated until there is no further loss in weight.

Use of the Gooch Filter. With the weighed crucible fitted in the adapter, and with gentle suction already applied, the liquid should be poured from the precipitate into the crucible; the precipitate may then be washed by decantation, transferred to the crucible, and the washing there completed. The rod used to transfer the liquid should reach nearly to the bottom of the crucible, in order not to stir up the asbestos, and the filtrate should be carefully examined for suspended particles of asbestos, and repassed through the filter if any are visible. The crucible with its contents is finally dried to constant weight, as already described. The increase represents the weight of the precipitate.

The convenience and efficiency with which precipitates can be prepared for the balance (with the repeated use of the same filter), leads to the use of crucibles of this type with practically all coarse-grained, crystalline precipitates; colloidal, gelatinous precipitates tend to stop up the pores of the filter.

With Gooch crucibles of platinum, set into closely fitting, shallow caps of platinum, the precipitates may readily be ignited at a high temperature; but in this case it is better to pack the crucible with a felt of platinum sponge. ${ }^{1}$ In this form, the crucible is known as a Munroe crucible, and, if properly prepared, the platinum felt is far more suitable for fine-grained precipitates than that of asbestos. Gold crucibles provided with platinum filters are cheaper, but should not be heated to a high temperature.

${ }^{1}$ For the preparation of such filters, see Journal of the American Chemical Sociely, vol. 3 I, p. $45^{6}$. 
Sources of Error. Since asbestos may absorb appreciable amounts of alkali, not removed by washing, solutions containing fixed alkalies should as a rule not be filtered through asbestos. Also, asbestos is slightly attacked by water and dilute acids; after the treatment indicated above, however, there is not much danger from this source. - Munroe crucibles, on the whole, give less chance for error.

The Theory of Washing Precipitates. The theory of washing precipitates should include the consideration of several factors, among which may be mentioned the phenomena of adsorption (in which the filter also takes part), and the tendency of precipitates to enter the wash liquid in colloidal form. But these factors have been discussed under precipitation. Aside from these considerations, there is the important question concerning the most effective method of washing precipitates.

Let us suppose that a precipitate is to be washed by decantation, and, for the sake of simplicity, that neither it nor the filter exercises any physical or chemical action on the dissolved salts. The mother liquid has been decanted as far as possible into the filter, and the latter allowed to drain.

Let $v$ cc. be the total volume of solution which remains in contact with the precipitate and filter, and $V$ cc. the volume of wash water added each time; and assume that the latter mixes uniformly with the liquid adhering to the precipitate and filter. Then, upon the addition of $V \mathrm{cc}$. of water, the total volume of liquid is $(V+v)$ cc. Further, let $C_{0}$ be the concentration in grams per cubic centimeter of the salts in the original solution; then the quantity contained in the $v$ cc. left in contact with the precipitate and filter is $v C_{0}$ grams. By the addition of $V$ cc. of water, the concentration is reduced to $C_{1}=\frac{v}{V+v} C_{0}$, and if this liquid is removed until only $v$ cc. are left, the quantity of undesirable salts present is reduced to $v C_{1}=\frac{v}{V+v} \cdot v C_{0}$ gm. A second addition of $V$ cc. of water gives the 
concentration, $C_{2}=\frac{v}{V+v} C_{1}=\left(\frac{v}{V+v}\right)^{2} C_{0}$, and the quantity of salts in the $v \mathrm{cc}$. left on draining is now

$$
v C_{2}=\frac{v}{V+v} \cdot v C_{1}=\left(\frac{v}{V+v}\right)^{2} v C_{0} \text { gm. }
$$

or, after $n$ washings, the quantity of undesirable salts has been diminished to the value,

$$
v C_{n}=\left(\frac{v}{V+v}\right)^{n} v C_{0} \mathrm{gm} .
$$

This formula expresses mathematically the self-evident fact that, for a given number of washings, the quantity of undesirable salts left behind will be the smaller, the more completely the precipitate and filter are drained, and the greater the volume of the wash water that is added each time. The formula enables us, however, to answer a less simple question; viz., What is the most efficient method of washing a precipitate with a given amount of wash liquid? Suppose, for example, we wish to use $150 \mathrm{cc}$. of wash liquid: is it better to wash six times with $25 \mathrm{cc}$. portions, or to wash ro times with $15 \mathrm{cc}$. portions? Let us assume that $\mathbf{C}_{0}=0$. I g. per cubic centimeter, and that $v=5 \mathrm{cc}$; then, in the two cases, the quantities of undesirable salts left behind will be $\left(\frac{5}{30}\right)^{6} \times 0.5=0.0000107 \mathrm{~g}$., and $\left(\frac{5}{20}\right)^{10} \times 0.5$ $=0.00000047 \mathrm{~g}$., respectively. Disregarding adsorption, which greatly decreases the efficiency of washing, ten washings with I5 cc. portions are 23 times as efficient as six washings with 25 cc. portions. Both methods of procedure will require approximately equal intervals of time, since, in either case, $150 \mathrm{cc}$. of liquid must run through the filter. It is much better to wash a precipitate many times with small portions of liquid, than a few times with larger portions. Each portion of wash liquid should be removed as far as possible by decantation and drainage, before the addition of a fresh portion.

Another factor to be considered is the temperature of the 
solution to be filtered. Since the rate of flow through a filter depends largely upon the viscosity of the liquid, and since the viscosity of water at $100^{\circ}$ is only one sixth that at $0^{\circ}$, it is well to filter and wash at a high temperature, unless there is good reason to the contrary.

Finally, in washing a precipitate on a paper filter, great care must be taken to wash the filter itself. Soluble salts are tenaciously held back at the upper edges of the paper, and therefore this part of the filter should receive especial attention. It is best to fill the filter each time, and, before refilling, to allow it to drain completely. Filters should be selected which are not too large.

\section{THE DRYING AND IGNITION OF PRECIPITATES}

Drying Ovens. There are on the market many types of drying ovens, heated by gas, by steam pipes, or by electricity, in which the temperature may be more or less accurately controlled. The oven consists essentially of a drying chamber, through which there is provided a slow circulation of hot air.

A precipitate is dried on the filter by placing the funnel containing both in a drying oven, at $90-100^{\circ}$, and leaving it there for a sufficient time. The funnel should be covered with a sheet of common filter paper, fastened in place by crimping its edges over those of the funnel. If the precipitate is suitable for weighing without ignition, e.g. silver chloride in a Gooch crucible, it should be dried to constant weight at a temperature considerably above the boiling point of water (in this case, at I $20-\mathbf{I} 30^{\circ}$ ), in order to remove the last traces of moisture from the filter as well as from the precipitate. (Before it is used, the packed Gooch crucible should, of course, be dried to constant weight at the same temperature.)

Many precipitates may, under proper precautions, be ignited without previous drying in an oven. But if such precipitates can be dried over night, or otherwise, without loss of time to the analyst, it is well to submit them to this process. 
The precipitate, folded within the filter, and placed at the bottom of the inclined open crucible, is heated gently, to avoid the sudden escape of steam. The drying is most safely accomplished by alternately applying and withdrawing the flame, the heat being applied to the lower side of the crucible, near its mouth. When the bundle is dry, the crucible is heated at the same point, but with a stationary flame, the temperature being gradually increased to the charring point of the paper; the volatile products should pass off without burning, since that would give rise to draughts within the crucible, with consequent danger of loss. After the dry distillation of the paper, in burning off the carbon, the flame should be applied to the bottom of the crucible, since this will insure a gentle circulation of air within the inclined vessel. The crucible may finally be heated to redness until the ignition is complete.

Some precipitates are reduced or otherwise affected by the hot carbon or reducing gases from the filter paper; e.g. silver chloride, lead sulphate, etc. are reduced to metal. Since, however, these metals are volatile only at very high temperatures, there is no loss in their case, and the metal can easily be converted into the original compound. In such cases, it is advisable to separate the precipitate as far as possible from the filter, and then to ignite the latter. The small quantity of reduced metal is moistened with a few drops of nitric acid, and the resulting nitrate converted into silver chloride with hydrochloric acid, or into lead sulphate with sulphuric acid, and the excess of acid expelled by cautiously heating the crucible. The bulk of the precipitate is then added, and the whole ignited.

Only in special cases may precipitates safely be heated over the blast lamp, and even then it is often preferable to use a large Meker burner.

Desiccators. In order to be weighed with accuracy, a freshly ignited object must first be allowed to cool ; but during this process it should be protected from the action of moisture, carbon dioxide, etc. It is therefore necessary to use a desiccator, - a 
special form of glass vessel, rendered air-tight by means of groundglass contact surfaces which are thinly coated with grease. ${ }^{1}$

For general analytical work, desiccators are usually charged with coarse granules of anhydrous calcium chloride, though sulphuric acid (spread out over fragments of pumice, to increase the active surface), solid potassium hydroxide, or quick-lime is sometimes used. Supported above the drying agent, there is a porcelain plate which is provided with holes of a size suitable for the reception of crucibles. Desiccators should be opened only when necessary, and promptly closed; the charge will then retain its efficiency for many months.

Crucibles. The most commonly used crucibles are of high grade porcelain. They withstand very high temperatures without appreciable change of weight, and are comparatively cheap. They are not suitable for fusions because most fluxes, particularly basic ones, attack the glaze as well as the porcelain. Even in the ignition of ordinary precipitates, in spite of careful washing, traces of fusible material usually remain present, and these in time roughen the glaze and destroy the usefulness of the crucible.

Crucibles more or less suitable for the ignition of precipitates are also made of alundum, and of fused silica.

Crucibles of platinum are often very desirable for ignitions; and for many fusions they are essential. Platinum melts at about $\mathrm{I} 770^{\circ}$ and does not soften enough to preclude its use at temperatures slightly below its melting point. ${ }^{2}$ It is soluble in liquids containing free chlorine, such as nitrate-chloride mixtures of acid reaction, and to a lesser degree in acid ferric chloride solutions. These facts should be borne in mind, in order to avoid injury to platinum vessels, and also in connection with the possible presence of platinum in certain analytical solutions.

1 A mixture made by melting together equal parts of vaseline and beeswax is very suitable.

2 An alloy called "palau," containing 80 per cent of gold and 20 per cent of palladium, is for some purposes a satisfactory substitute for platinum. It melts, however, at $1370^{\circ}$, much lower than platinum. 
Platinum easily alloys with most metals, and for that reason it should not ordinarily be heated in contact with metals, or with compounds of easily reducible metals, - never, if carbon or reducing gases are also present. When heated with carbon, platinum slowly takes this up and becomes brittle. The crucible, therefore, should not be heated in a reducing flame; the flame should be non-luminous, and so adjusted that the crucible is wholly above the inner cone. Burners of the Meker type are very suitable for the heating of platinum ware. "Unknown " substances should not be heated in platinum ware, nor should phosphates or arsenates be heated under reducing conditions; these elements, as well as phosphides and arsenides are ruinous in their action upon platinum.

Platinum ware should be kept clean and well polished. For this purpose moistened precipitated silica is very satisfactory; it removes most impurities and polishes the platinum without the loss of more than a very few milligrams. Potassium bisulphate, fused within the vessel, is an efficient cleansing agent; but the melt should be poured out while still liquid.

Only triangles of platinum, quartz, or pipeclay should be used with platinum crucibles, and hot platinum vessels should not be set down on foreign metals. The crucibles should be handled while hot only with platinum-shod tongs. ${ }^{1}$

1 Modern platinum ware is often inferior in quality to that on the market some years ago, and the cause has been the subject of special inquiry by a committee of the American Chemical Society. The main objections are: "(I) Undue loss of weight on ignition; (2) undue loss on acid treatment, especially after strong ignition; (3) unsightly appearance of the surface after strong ignition, especially after the initial stages of heating; (4) adhesion of crucibles and dishes to triangles, sometimes to such an extent as to leave indentations on the vessel at the points of contact with the triangle, even when complete cooling has been reached before the two are separated; (5) alkalinity of the surface of the ware after strong ignition; (6) blistering; and (7) development of cracks after continued heating." It is the general opinion that the trouble arises from the working of scrap platinum into chemical ware. The main difficulties here mentioned are not characteristic of platinum ware from some of the best manufacturers.

The committee recommends that purchasers specify that platinum ware must show no marked uneven discoloration on heating, must give no test for iron after 


\section{THE EVAPORATION OF LIQUIDS}

Evaporations are most efficiently carried out in wide, shallow dishes or casseroles, which expose a large surface of liquid to the air. Great care must be taken to prevent the loss of material by spattering, due to the use of too high a temperature, or from evaporation to a low volume accompanied by the crawling of salts. Solutions evolving gases, or which are to be boiled, should always be covered. And liquids which contain precipitates, or other solid matter, should be heated cautiously and with stirring; the presence of solid matter at the bottom of a liquid often causes violent bumping, and this may even result in the destruction of the vessel.

The evaporation of aqueous solutions rarely requires a temperature higher than $95^{-100^{\circ}}$; the use of the steam bath, with which mechanical losses due to boiling and bumping need not be feared, is in general advisable with aqueous solutions. In evaporations on the steam bath, a watch glass should be placed above the vessel to prevent the entrance of foreign matter; but, by means of a glass triangle, it should be elevated above the rim, in order to permit the free exit of vapor.

If a large volume of liquid is to be evaporated, the dish need not necessarily contain it all. Fresh portions may be added from time to time, as room is made in the dish by evaporation.

Liquids should be transferred from one vessel to another only with the aid of a glass rod, held tightly against the lip of the container. In the case of vessels without lips, in order to prevent the liquid from running down outside, the outside edge should be coated with a thin film of vaseline.

In quantitative work, as few transfers of liquid as possible should be made. Such transfers must be made quantitative by repeatedly washing the former container with small portions of wash liquid; besides being the most effective, this method of washing also keeps the total volume within bounds.

heating for two hours, and that the rate of loss per hour at $1100^{\circ}$ over a period of four hours shall not exceed $0.2 \mathrm{mg}$. 


\section{THE VOLUMETRIC MEASUREMENT OF LIQUIDS ${ }^{1}$}

In a well-equipped weighing room, measurements can be made with a precision often greater than is necessary ; for the errors involved in the preparation of a troublesome precipitate may impair the value of an exact weighing. Although the measurement of volume, in volumetric analysis, is not apt to be so precise and reliable as the measurement of weight, ${ }^{2}$ yet the results of volumetric processes, based on suitable reactions, are frequently more trustworthy than those of gravimetric processes, because the volumetric process for the determination of the substance is less liable to error. With proper precautions many volumetric processes yield excellent results ; and, especially in technical work, where time is an essential factor, volumetric processes are very often used in preference to gravimetric. In order, however, that dangerous errors may be eliminated, it is essential that the analyst should have a thorough understanding of the precautions necessary for the attainment of a high degree of accuracy.

Volumetric Apparatus. The exact volumetric measurement of liquids involves the use of certain special forms of apparatus.

Burettes are graduated glass tubes of uniform, small diameter, for the measurement of variable volumes of liquid delivered by them when in a vertical position. The outflow is controlled either by a glass stopcock, or by means of a short rubber tube provided with a pinchcock (or other suitable device), which connects the end of the tube with a glass outlet, or tip. The glass

1 For more detailed information on this subject, see Bulletin of the Bureau of Standards, vol. 4, pp. 553-601 (1908).

${ }^{2}$ Even this difficulty can be obviated by the use of weight burettes; i.e. of burettes of such construction as to be readily weighable both before and after the removal of the quantity of solution required for the completion of the given reaction. The difference gives the weight of solution required, and, provided the solution has been standardized by the same method, the quantity of the substance under investigation can be readily calculated. In this way, if based on suitable reactions, exceedingly exact determinations can be executed. (A similar weight burette should always be used as a counterpoise.) 
stopcock requires the use of a lubricant, as vaseline, to permit easy regulation; and the rubber joint has the disadvantage that it is attacked by some solutions, which in consequence suffer a change in concentration, - e.g. rubber stopcocks must be avoided with permanganate and iodine solutions.

Transfer Pipettes are very narrow tubes which are designed to deliver specific volumes of liquid; an enlargement at the center, however, greatly reduces the length required, and a mark on the tube above this enlargement indicates the level to which the instrument must be filled in order to deliver the indicated volume of liquid. Pipettes are filled by suction and are allowed to deliver, from a vertical position, by the action of gravity. Owing to the small bore of the tube, the pipette is capable of a high degree of accuracy; but errors of manipulation may render the measurements inexact.

Measuring Flasks are usually employed in the measurement of relatively large volumes. The neck should be of uniform bore, should extend well above and below the graduation mark, and should be small enough to permit of accurate reading, but sufficiently large to render filling and emptying easy. For the most accurate work, the flask is graduated for containing the nominal volume of liquid.

Measuring Cylinders are glass vessels provided with a broad base, or foot, and with a lip for pouring. They are graduated to contain or deliver variable volumes of liquid, and are used for rough measurements only.

Sources of Error in the Use of Volumetric Apparatus. In the use of volumetric methods, the following sources of error must be fully reckoned with if the results are to be reliable.

Water in the Apparatus. The apparatus must nearly always be washed with water before use, and any water retained would of course alter the concentration of the solution to be measured. While the apparatus might be dried before use, it is far more convenient, and just as accurate, to wash it out with small portions of the solution, and to discard the washings. 
Drainage or Afterflow. When an aqueous liquid is permitted to flow rapidly from a burette, or pipette, some of the liquid adheres to the inner surface, and only gradually runs down to the liquid below. To avoid errors from this source, the rate of outflow must be limited by the size of the outlet, or a sufficient time interval must be allowed to elapse before the reading is made.

In the case of transfer pipettes, the outlets should be made of such size that the free outflow shall require at most a minute, but not less than 15,20 , and 30 seconds, respectively, for 5 , Io, and 50 pipettes. The free outflow from a burette should never take much more than 3 minutes, nor less than 90 and 50 seconds for 50 and $30 \mathrm{cc}$. burettes, respectively. Burette and pipette tips should be made with a gradual taper of $2-3 \mathrm{~cm}$.; a sudden contraction at the orifice is not permissible, and the tip should be well finished.

Grease Films. Certain substances, especially grease, adhering to the walls of measuring vessels prevent their uniform wetting; the tendency of water to collect into droplets, instead of flowing uniformly over the glass, indicates the presence of a film of grease. Imperfect wetting not only distorts the meniscus, but, by influencing the residue adherent to the walls, it also gives rise to irregularities in the delivery of liquid.

When cleaning accurately calibrated measuring vessels with sulphuric acid-dichromate solution, they should be filled with the cold solution and allowed to stand overnight, or longer. The use of hot liquids in such vessels is to be avoided on account of the possible thermal after effect on the glass; while for certain kinds of glass this effect may be negligible, we have no assurance that it is so.

Parallax. In apparatus in which the volume is limited by a meniscus, the reading or setting is made, when possible, on the lowest point of the meniscus. This point lies at the center of the tube; the reading should be made, therefore, with the tube in a vertical position, and with the eye so located 
that the line of sight is perpendicular to the main axis of the tube.

In making this reading, it is well to place a dark-colored screen immediately below the meniscus; this will render the profile of the meniscus dark and clearly visible against a light background. A convenient device for this purpose is a collar-shaped section of black rubber tubing, cut open at one side, and of such a size as to clasp the tube firmly.

Variations in Temperature. The volume occupied by a given weight of water, as well as the capacity of the measuring vessel, is dependent upon the temperature; and the error involved in the measurement of the volume of a given mass of water, at any other temperature than the standard one, is due to the joint effect of the changed capacity of the vessel and the changed volume of the liquid.

The coefficient of cubical expansion of ordinary glass is very close to 0.000025 ; but the volume change of the water is much greater than that of the glass measuring vessel, and also much less uniform from degree to degree. The factors by which a volume of water, measured at temperatures ranging from ro$29^{\circ}$ in a vessel calibrated for $20^{\circ}$, must be multiplied in order to obtain the true volume occupied by the liquid at $20^{\circ}$, are given in the following table:

\begin{tabular}{|c|c|c|c|c|c|}
\hline & \multicolumn{5}{|c|}{ Temperature of the Water } \\
\hline TENS UnITS & $\mathbf{0}$ & 1 & 2 & 3 & 4 \\
\hline 1 & 1.00124 & 1.00117 & I.00109 & 1.00100 & I.00089 \\
\hline 2 & 1.00000 & $0.9998 \mathrm{I}$ & $0.9996 \mathrm{I}$ & $0.9994 I$ & 0.99919 \\
\hline
\end{tabular}

\begin{tabular}{|c|c|c|c|c|c|}
\hline & \multicolumn{5}{|c|}{ Temperature of the Water } \\
\hline TENS Units & 6 & 6 & 7 & 8 & 9 \\
\hline 1 & 1.00077 & 1.00064 & 1.00049 & 1.00034 & 1.00018 \\
\hline 2 & 0.99896 & 0.99873 & 0.99848 & 0.99822 & 0.99798 \\
\hline
\end{tabular}


If the prevailing temperature does not differ by more than, say, $3^{\circ}$ from the standard, this correction may ordinarily be omitted. In the case of solutions of $0.2 \mathrm{~N}$ concentration, or less, the corrections differ so little from those for pure water that the factors given in the table may be used without appreciable error.

In order to illustrate the use of such factors, let us suppose that, in the standardization of a solution, a burette graduated correctly for $20^{\circ}$ is used at an actual temperaure of $27^{\circ}$, and that the indicated volume of solution withdrawn is $28.75 \mathrm{cc}$. Then the true volume at $20^{\circ}$ of this quantity of liquid is $28.75 \times$ $0.99848=28.70 \mathrm{cc}$. And, if a determination is later made with this solution at, say, $17^{\circ}$, and the indicated volume used is $28.68 \mathrm{cc}$., then the true volume at $20^{\circ}$ is $28.68 \times \mathrm{I} .00049=28.70 \mathrm{cc}$. That is to say, the same quantity of reagent is contained in an apparent volume of $28.75 \mathrm{cc}$. at $27^{\circ}$, or in an apparent volume of $28.68 \mathrm{cc}$. at $\mathrm{I} 7^{\circ}$, as is contained in an actual volume of $28.70 \mathrm{cc}$. of the solution at $20^{\circ} .{ }^{1}$

Different Units of Volume. Unfortunately, a number of different "liters" have been suggested for use in volumetric analysis. The normal liter, that is, the volume occupied by a kilogram of water, weighed in a vacuum and measured at $4^{\circ}$, would manifestly be out of the question if it had to be determined in that way. The so-called "Mohr liter" is the volume occupied by a kilogram of water when weighed in the air with brass weights at a temperature of $17.5^{\circ}$; but this volume varies with the atmospheric conditions. Other "liters" involving measurements at $15^{\circ}, 15.5^{\circ}$, or $20^{\circ}$ have been suggested by various chemists.

It matters little, in analytical work, which liter is adopted, but it is of the greatest importance to have the pipettes, burettes, and measuring flasks rigorously consistent with one another. This matter requires especial emphasis, since apparatus, if not speci-

1 At $27^{\circ}$ the actual volume of the liquid measured is a shade greater than $28.75 \mathrm{cc}$, and at $17^{\circ}$ it is a shade less than $28.68 \mathrm{cc}$. The measuring vessel is larger at $27^{\circ}$, and smaller at $17^{\circ}$, than it is at $20^{\circ}$. 
fically ordered, may be supplied by dealers, at different times, graduated according to different systems; and mixed graduations may thus come into the hands of an individual analyst. As an example of the magnitude of the errors which might thus be introduced, it should be noted that the normal liter is related to the Mohr liter as 1000: 1002.3. .

Much of the graduated apparatus on the market bears no mark by means of which the unit of volume represented can be recognized, and even when this is clearly designated the percentage error represented may be large. It is not advisable, therefore, to use any piece of graduated apparatus, unless its actual value is well known.

Owing to the great difficulty in measuring directly the relation between cubic capacity and the unit of length, the International Committee of Weights and Measures defines the liter as " the volume occupied by the mass of one kilogram of pure water at its maximum density under normal atmospheric pressure." This is almost exactly rooo cc. ${ }^{1}$ and for all practical purposes may be regarded as such.

It is now customary to use this true liter as the standard, but of course it is out of the question to weigh a kilogram of water at $4^{\circ}$ in a vacuum; some convenient temperature preferably the average working temperature of the laboratory - must be selected, and the necessary corrections made. If a liter flask is marked correctly at $20^{\circ}$, this means that at $20^{\circ}$ it will contain a mass of water ( $998.234 \mathrm{~g}$.) which occupies a volume equal to that occupied by 1000 grams of pure water at $4^{\circ}$. This quantity of water, if weighed with brass weights in air of mean humidity, at $20^{\circ}$ and $760 \mathrm{~mm}$., has an apparent weight of 997.18 grams.

The Calibration of Volumetric Apparatus. The weight "of brass (brass weights) which will be required to counterbalance one liter of pure water must be calculated from the temperature of the water and the density of the air. The following table

1 About $1000.029 \mathrm{cc}$. 
indicates, for temperatures of the water (and room) ranging from $15^{-29^{\circ}}$, how many milligrams less than rooo grams a quantity of water will weigh which is sufficient to fill to the mark a I-liter flask correctly calibrated for $20^{\circ}$, the weighing being carried out in air of 50 per cent humidity at $760 \mathrm{~mm}$. pressure (unreduced). ${ }^{1}$

\begin{tabular}{|c|c|c|c|c|c|c|c|c|c|c|}
\hline TENS UNITS & 0 & 1 & 2 & 3 & 4 & 5 & 6 & 7 & 8 & 9 \\
\hline 1 & - & - & - & - & - & 1950 & $\frac{2100}{2260}$ & $\frac{2440}{2620}$ \\
\hline 2 & 2820 & 3030 & 3240 & 3470 & 3710 & 3960 & 4210 & 4480 & 4760 & 5040 \\
\hline
\end{tabular}

If such a flask is filled to the mark with water of $22.4^{\circ}$, for example, the water will under the conditions of the table require a counterpoise of 1000-3.332 $=996.668$ grams.

The determination of the capacity of a measuring flask is carried out by weighing the water contained in it, while the volume of water delivered by a burette or pipette is determined by weighing this water after its delivery into another vessel. The temperature of the water, which should be the same as that of the room, should be taken immediately before and after the experiment.

In the calibration of a flask, the dry flask is placed upon the right-hand pan of the balance, together with the nominal weight of its capacity, i.e. with as many grams as it is supposed to contain cubic centimeters, and then tare material is placed upon the left-hand pan until the balance is brought into equilibrium. The weights are then removed from the right-hand pan, the flask is filled to the mark with water, and weights are added until the balance is again in equilibrium. The nominal capacity weight, minus the additional weight which is required upon the right-hand pan in order to reëstablish equilibrium, is equal to the weight of the water in the flask.

In the case of burettes and pipettes, a covered beaker is placed

1 These values depend upon the specific gravities of the water and the brass, the density of the air, and the coefficient of cubical expansion of the glass vessel. 
upon the right-hand pan, together with the nominal weight in grams of the volume to be delivered, after which the balance is brought into equilibrium by the addition of tare material to the left-hand pan. The water is then allowed to run into the beaker, which is replaced upon the right-hand pan. The subsequent procedure is the same as that described above.

The difference between the additional weight required to reëstablish equilibrium and that calculated from the above table indicates directly the error of the vessel. If, for example, it be found necessary, in order to reëstablish equilibrium in the case of a 500-cc. flask filled to the mark with water of $22.4^{\circ}$, to add $1.832 \mathrm{~g}$., instead of the calculated $\mathrm{r} .666 \mathrm{~g}$., then it follows that the vessel is o.r $66 \mathrm{cc}$. too small. On the other hand, in testing the $25 \mathrm{cc}$. segment of a $30-\mathrm{cc}$. burette, at a temperature of $17^{\circ}$, the additional weight required on the right-hand pan should be $(25 \times 2260) \div 1000=57 \mathrm{mg}$.; if, instead of this, it be found that an additional weight of $15 \mathrm{mg}$. is required on the left-hand pan, then the $25 \mathrm{cc}$. segment is $57 \mathrm{mg} .-\left(-\mathrm{r}_{5} \mathrm{mg}\right.$. $)=0.072 \mathrm{cc}$. too large. ${ }^{1}$

In the calibration and use of burettes, the liquid should in general be allowed to flow from the zero mark to some second level in the burette.

\section{THE PREPARATION OF SAMPLES FOR ANALYSIS}

It is not easy to give general rules for the preparation of substances for analysis, because it is necessary to proceed differently in different cases. In all cases, however, the samples should promptly be transferred to tightly stoppered bottles or weighing tubes.

In technical analyses, for the purpose of determining the commercial value of an article, or of controlling processes of manufacture, materials must be analyzed as they are. But, in every case, especial care should be taken to make up a sample

1 This error is too large to be tolerated. 
which will represent as nearly as possible the average composition of the whole lot.

If, on the other hand, it is desired to determine the atomic composition of a compound, it is necessary to select or prepare pure material for analysis. This may seem simpler than it really is. Many compounds absorb or give up moisture upon exposure to the air, and their treatment should vary with their nature, as illustrated in the following cases. Salts such as $\mathrm{Na}_{2} \mathrm{SO}_{4}$. I० $\mathrm{H}_{2} \mathrm{O}$ and $\mathrm{Na}_{2} \mathrm{CO}_{3}$. Iо $\mathrm{H}_{2} \mathrm{O}$, which effloresce in ordinary air, may be dried, after recrystallization, by strongly pressing the powdered material between several layers of filter paper, the paper being renewed as long as moisture continues to be taken up; $\mathrm{MgSO}_{4} \cdot{ }_{7} \mathrm{H}_{2} \mathrm{O}$ and $\mathrm{NaKC}_{4} \mathrm{H}_{4} \mathrm{O}_{6} \cdot 4 \mathrm{H}_{2} \mathrm{O}$, which do not lose water of constitution in ordinary air, may be spread out upon filter paper, covered with another sheet, and allowed to dry at the ordinary temperature. Compounds such as $\mathrm{HFe}\left(\mathrm{SO}_{4}\right)_{2} \cdot 4 \mathrm{H}_{2} \mathrm{O}$ and $\mathrm{CaC}_{4} \mathrm{H}_{4} \mathrm{O}_{6} \cdot \mathrm{H}_{2} \mathrm{O}$, which do not effloresce in artificially dried air, but which undergo chemical change at $100^{\circ}$, may be conveniently dried in a desiccator, over calcium chloride. Substances, as $\mathrm{KHC}_{4} \mathrm{H}_{4} \mathrm{O}_{6}$, sugar, etc., which readily give up hygroscopic moisture at $100^{\circ}$, without other alteration, are best dried in an oven at that temperature; while $\mathrm{K}_{2} \mathrm{PtCl}_{6}$, which retains moisture, or dries only slowly at $100^{\circ}$, but which decomposes below a red heat, should be dried in an oven at, say, $\mathrm{I}_{3} 0^{\circ}$. Finally, substances such as $\mathrm{NaCl}, \mathrm{Na}_{2} \mathrm{SO}_{4}$, etc. may be given a preliminary drying, in a covered vessel, at $\mathbf{I} 30^{\circ}$, or higher, to prevent decrepitation, and then be ignited, more or less strongly, depending upon their nature. In every case, the sample should be dried, without decomposition, to constant weight.

Substances used in testing the accuracy of analytical processes, or in standardizing volumetric solutions, must also be extremely pure. In fact, compounds are generally favored which are non-hygroscopic, and which may readily be prepared in a pure condition; if possible, it is well to select compounds 
which normally do not contain water of crystallization. Many salts can be obtained sufficiently pure in the market; but their purity should never be accepted on faith. If tests indicate the presence of impurity, and, often, if the salt contains water of crystallization, the material should be recrystallized.

For this purpose, a convenient weight of the salt is dissolved in the least possible quantity of hot water, using a quantity of water not quite sufficient to dissolve the whole lot; the hot solution is poured into a fluted filter, held in a stemless funnel, and the filtrate is received with continuous stirring in a beaker, which itself is immersed in cold water, in a larger vessel. The rapid cooling and constant stirring cause the formation of a fine crystalline powder, which is almost free from inclosed mother-liquor. The crystalline powder is filtered off in a funnel containing a perforated platinum cone, the adhering motherliquor being removed by suction or in a centrifuge. Two such recrystallizations will nearly always suffice. According to the nature of the substance, it is dried in the air at a specific temperature, or in a desiccator, to constant weight.

Concerning the preparation of samples for analysis by beginners in quantitative analysis, the reader should consult the Appendix. 


\section{PART II}

\section{GRAVIMETRIC ANALYSIS}

\section{EXERCISES WITH THE BALANCE}

BEFORE beginning work at the balance, read carefully the rules given on pp. 9-II of Part I, and observe them always.

Determination of the Rest-Point. Determine the rest-point of the unloaded balance, according to the method on p. Ir. If this is not more than one division from the center of the scale, the balance may be used by the student; otherwise it will be adjusted by an instructor, upon request. The beginner should not attempt this adjustment.

Determination of the Weight of an Object. Clean two porcelain crucibles, rinse them with distilled water, and allow them to drain. Place each crucible upon a pipestem triangle, supported upon a tripod, and heat with the colorless flame of a Bunsen or Tyrill burner, - gently at first, and then to a red heat. Allow the crucibles to cool off somewhat, but while still warm, place them in a desiccator, using the crucible tongs. ( $A$ piece of apparatus which has been ignited, or dried, for weighing must not be touched by the hand before it has been weighed.) $\mathrm{Al}-$ low the crucibles to cool in the desiccator for at least 20 minutes.

Now with the crucible tongs place a crucible on the left-hand pan of the balance, and, by means of the forceps in the weight box, place weights on the right-hand pan until they balance the crucible to within $0.005 \mathrm{~g}$. Begin with a weight which you think will approximately balance the object, lower the balance beam, and gently release the pan supports. It will then be seen which side is the heavier. Finally adjust the rider, so that, 
when the beam is swinging freely, the pointer swings equal distances on both sides of the rest-point. Always try the weights in the order in which they occur in the box, beginning with the heavier ones, and using the rider for weights smaller than 5 or Io milligrams, according to the number of large divisions on the beam.

As soon as the object appears to be balanced, raise and lower the beam, and make another observation. Read the weight of the crucible by noting in order the vacant spaces in the box, beginning with the largest missing weight; and check this reading as the weights are returned to the box. Be sure also to note the weight recorded by the rider, and then lift it from the beam. Always record the weight, in pencil as it is first read, and in ink after it has been checked, and always in the record book.

In this manner, weigh the two crucibles separately, and then weigh them together, entering all three results in the notebook. (In connection with the keeping of records, see the remarks on p. 5.) The sum of the separate weights should agree closely with the result obtained upon weighing both crucibles together, - within, say, $0.0002 \mathrm{~g}$. 


\section{THE DETERMINATION OF CHLORINE IN A SOLUBLE CHLORIDE}

The sample may be pure sodium chloride, or it may be an artificially prepared mixture of sodium chloride and sodium carbonate.

Method. The aqueous solution of the chloride is acidified with nitric acid and treated with silver nitrate in excess. The chlorine is quantitatively precipitated as silver chloride, which is filtered off, washed, dried, and weighed. Other acids which yield silver salts insoluble in nitric acid must of course be absent.

$A$. A Paper Filter is Used: Procedure. Carefully wipe off the stoppered sample tube, without directly touching it, and weigh it accurately to o.r mg., entering the weight in the notebook (see p. 5). With the tube held just above a clean $300 \mathrm{cc}$. beaker (labelled "I"), remove the stopper, and, without loss of material in the form of dust or otherwise, carefully transfer to the beaker $0.2-0.3 \mathrm{~g}$. of the sample. Replace the stopper, weigh again to o.I $\mathrm{mg}$., and enter the result in the notebook. The first weight minus the second gives the weight of sample taken. Weigh out a second $0.2-0.3 \mathrm{~g}$. portion into another beaker (labelled "II "), entering the data as before. (Be sure always to cover the beakers promptly.)

Treat each sample as follows: Add roo-r25 cc. of distilled water, stir, and acidify with nitric acid; add the acid slowly with stirring until blue litmus paper indicates an acid reaction when touched with the moist stirring rod. Assuming the material to be sodium chloride, add slowly with stirring a volume of silver nitrate solution $4^{-5} \mathrm{cc}$. in excess of the calculated quantity (for the strength of the reagents, see the Appendix). Cover the beaker with a watch glass, and, with occasional stirring, stowly heat the solution to boiling. This treatment will cause the precipitate to coagulate and settle, leaving the liquid clear. The precipitate should not be exposed to direct sunlight, and great care should be taken to avoid the loss of material. Finally, add a little silver nitrate solution to the hot supernatant liquid, 
to test for complete precipitation; if a precipitate forms, add $5 \mathrm{cc}$. more, stir, allow to settle, and test again.

Prepare two $9 \mathrm{~cm}$. " ashless" filters (see p. 31 ), and decant the hot liquid through the filter in each case, leaving the precipitate mostly in the beaker. The filtrate should be free from turbidity; otherwise refilter it through the same paper. (Finally, pour the clear filtrate into the bottle for "Silver Residues.") With clean receptacles for the washings, wash each precipitate twice by decantation with Io cc. portions of hot water (to which it is well to add 3-4 drops of nitric acid), running the washings through the filters, and then transfer each precipitate to the corresponding filter. This transfer is made by means of a stream of hot water from the wash bottle, the adhering particles being loosened with the aid of a "policeman" (see p. 33). Wash the precipitates and filters with hot water until $5 \mathrm{cc}$. of the washings show no turbidity whatever with I drop of 6-normal hydrochloric acid. After the complete drainage of the filters, cover each funnel with an ordinary filter paper, crimping its edges over the rim of the funnel, and place it (numbered according to the notebook record, and bearing the student's name and desk number) in a drying oven, at $90-100^{\circ}$; allow the filters and precipitates to dry completely.

Now, in a quiet place, open each filter over a smoothly trimmed square of black glazed paper, about 6 inches in diameter, and with great care to avoid loss (by finally rubbing the folds of the paper gently together), transfer the precipitate to the center of the glazed paper; do not rub off any fibers from the paper. Cover the material on the paper with an inverted funnel or watch glass for protection.

With the paper refolded flat, bend the top over and $c$... the fold; then roll the paper into a small bundle and place it weighed porcelain crucible. Place the crucible on its side, upo a triangle, and ignite gently until the paper is decomposed. Then, with the flame at the back of the crucible, ignite strongly until the carbon is consumed. (See Part I, pp. 38-39.) After 
cooling, add 2 drops of 6-normal nitric, and $\mathrm{I}$ of hydrochloric acid, and heat gently, with great caution to avoid loss, until the acids are expelled. Allow to cool.

Place the cold crucible upon a piece of glazed paper (to catch any material that might be spilled), and transfer to it the main quantity of the silver chloride, brushing the last traces of the salt into the crucible with a small camel's hair brush. Now add 2 drops of nitric, and I of hydrochloric acid, expel the acids (observe caution), and then slowly raise the temperature until the salt just begins to fuse; at that point, remove the flame. Place the warm crucible in a desiccator, allow it to cool, and then weigh it. Without the acids, repeat the heating, etc. until the last change in weight observed does not exceed $0.2 \mathrm{mg}$. (Finally, place the silver salt in the bottle for silver residues.)

From the weight of the silver chloride yielded by each sample, calculate the percentage content of chlorine.

Notes. - I. The solution is acidified with nitric acid in order to prevent the formation and precipitation of substances, as silver oxide, carbonate, phosphate, etc., which are insoluble in water but soluble in nitric acid. The acid also helps to coagulate the precipitate.

2. It is safer not to boil the acidified solution until the silver nitrate has been added; otherwise a trace of hydrochloric acid might be oxidized by nitric acid and the liberated chlorine partially volatilized. While not at all likely at so low an acid concentration, it is nevertheless needless to run the risk of this possible action.

3. Silver chloride is decomposed slightly by direct sunlight, with the loss of chlorine; this action is much less in diffused light, and can be remedied by the acid treatment indicated. In diffused daylight, however, the error from this source is really too insignificant to affect the accuracy of the determination.

4. The precipitate is washed, before drying, in order to free it from the non-volatile soluble salts present in the solution. The washing is to be discontinued as soon as $5 \mathrm{cc}$. of the washings give no turbidity upon the addition of chloride-ion; only one or two drops of hydrochloric acid should be used in this test, owing to the solubility of silver chloride in concentrated chloride solutions. The wash water should be hot in order to keep the precipitate coagulated; this is still more assured if the water is very slightly acidified with nitric acid. 
5. Silver chloride itself is practically insoluble in cold water; slightly over I mg. per liter (i.e. I part per million of water) at $20^{\circ}$. Owing to the common-ion effect, the solubility is still less in the presence of a slight excess of either silver-, or chloride-ion, - e.g. in very dilute silver nitrate or hydrogen chloride solution. In hot water the salt is more soluble (about 22 parts per million, at $100^{\circ}$ ); but, fortunately, the speed of solution is so slow that the precipitate may be thoroughly washed with hot water, without undue error.

6. Silver chloride should not be ignited with the paper, because it would be largely reduced to metal $(\mathrm{AgCl}+$ " $\mathrm{H}$," from the paper $=\mathrm{Ag}+\mathrm{HCl})$. The small quantity which must necessarily be ignited with the paper is probably all reduced; but it can with certainty be changed back to chloride, without loss.

Silver chloride is also reduced by zinc and dilute sulphuric acid to metallic silver, and the salt adhering to the crucible after ignition can be removed by means of this action. Upon fusion with sodium carbonate, owing to the unstable nature of silver carbonate and oxide, silver chloride readily yields metallic silver.

7. Like most substances, silver chloride is appreciably volatile somewhat above its melting point. It is not safe, therefore, to fuse the precipitate over an ordinary burner; there is too much danger of overheating. If heated slowly, however, only to incipient fusion (around the edges), no error need be feared at this point.

8. Silver-ion has a great tendency to enter into the formation of complex ions, as $\left[\mathrm{Ag}\left(\mathrm{NH}_{3}\right)_{2}\right]^{+},\left[\mathrm{Ag}(\mathrm{CN})_{2}\right]^{-},\left[\mathrm{AgS}_{2} \mathrm{O}_{3}\right]^{-}$, and, in consequence, the socalled insoluble salts of silver often enter into solution upon the addition of ammonia, or of an alkali cyanide or thiosulphate. Such facts as these must be acquired and kept in mind by the analytical chemist; chemical knowledge can be of the greatest service in combating difficulties, while lack of knowledge is often the occasion of trouble.

9. The silver residues should conscientiously be turned in by the student. Assuming that duplicate determinations require on the average $55 \mathrm{cc}$. of 0.2-normal silver nitrate solution, and taking into account the analyses which have to be repeated, the residues returnable by a class of 100 students should contain about $150 \mathrm{~g}$. ( $5 \mathrm{oz}$.) of metallic silver. This can easily be recovered and transformed into pure silver nitrate, for future use by other students.

\section{B. A Gooch Crucible is Used: Procedure. Weigh out two} samples of the substance, of about $0.25 \mathrm{~g}$. each, and convert the chloride into silver chloride as described in procedure $A$. Mean- 
while, prepare two Gooch crucibles, following the directions on pp. 33-35; and finish the analysis according to the details there given.

Notes. - r. Silver itself (e.g. as $\mathrm{AgCl}$ ), as well as bromides, iodides, cyanides, thiocyanates, etc., may be determined in a similar manner, with the use of Gooch crucibles. The disadvantage of using a paper filter, e.g. with silver bromide or cyanide (requiring the conversion of reduced silver back to the bromide or cyanide), is at once apparent.

2. Chlorates, etc., may be determined as silver halides by first reducing them to the oxygen-free salts, and precipitating these with silver nitrate.

3. For the principles involved in the determination of these substances when two or more of them are present in the same sample, the student is referred to Part V, Problems 37, 38, 90, 91, and 93. 


\section{THE DETERMINATION OF IRON AND OF SULPHUR IN A SOLUBLE SULPHATE OF IRON}

The sample may be pure ferrous ammonium sulphate, pure ferric alum, or an artificially prepared mixture of anhydrous ferric sulphate, sodium carbonate, and potassium sulphate. This mixture is readily soluble in dilute hydrochloric acid.

Method. The sample is dissolved in water, with the addition of hydrochloric acid, after which the iron is oxidized to the ferric condition, unless it is already wholly present in that state. The iron is then separated, by double precipitation with ammonium hydroxide, as ferric hydroxide. The precipitate is ignited, and weighed as ferric oxide.

From the combined filtrates and washings, which must have a large volume, and which must be free from nitrates, etc., the sulphate is precipitated by means of a dilute solution of barium chloride. The precipitate is ignited, and weighed as barium sulphate.

$A$. Procedure for the Determination of Iron. Weigh out into dry $500 \mathrm{cc}$. beakers two portions of about I gram each, and add to each portion $50 \mathrm{cc}$. of water and ro cc. of 6-normal hydrochloric acid, keeping the beakers covered with watch glasses to prevent loss by effervescence. Heat the solutions to boiling, and treat each as follows: Add r6-normal nitric acid (before doing this, see Note 2), drop by drop with stirring, until the darkened liquid clears to a yellow, being careful not to add more than I cc. altogether. Boil for 3 minutes, add roo cc. of water, heat again to boiling, and add rapidly with stirring $25 \mathrm{cc}$. of 6-normal ammonium hydroxide solution. Digest the mixture, which should smell strongly of ammonia, with heating and occasional stirring, until the precipitate is well coagulated; allow to settle; and decant the hot liquid through a 9-cm. ashless filter, washing the precipitate twice by decantation with $25 \mathrm{cc}$. portions of hot water, and leaving it mainly in the beaker. (Neutralize the filtrate and washings with hydrochloric acid, 
and begin the evaporation of the liquid at this point, as directed under the determination of sulphur. See p. 4.)

Dissolve the precipitate by slowly pouring over the filter a boiling mixture of $5 \mathrm{cc}$. of water and ro cc. of 6-normal hydrochloric acid, and receiving the filtrate (and washings) in the beaker containing the bulk of the precipitate, which also will completely dissolve. After thoroughly washing the filter, tear it into small bits and add these to the ferric chloride solution. Dilute to roo cc., heat to boiling, and add as before $25 \mathrm{cc}$. of 6-normal ammonia. Digest for some time, near the boiling temperature, and decant the hot liquid through a fresh filter, washing the precipitate with hot water, at first by decantation and finally on the filter, until $5 \mathrm{cc}$. of washings give no cloudiness with a drop or two each of nitric acid and silver nitrate solution. (Neutralize the filtrate and washings as before, add the liquid to that from the first precipitation, and allow the evaporation to continue.)

Ignite the precipitate, together with the filter paper, in the usual manner in an inclined platinum or porcelain crucible; after the decomposition of the paper, increase the flame to the full, and, with free access of air, heat for 20 minutes. Cool in the desiccator and weigh; remember to repeat this operation to the attainment of constant weight. Report the percentage of iron in the sample.

Notes. - r. In contact with air, the iron in ferrous salt solutions is slowly oxidized by dissolved oxygen $\left(4 \mathrm{Fe}^{++}+\mathrm{O}_{2}+2 \mathrm{H}_{2} \mathrm{O}=4 \mathrm{Fe}^{+++}\right.$ ${ }^{+} \mathrm{OH}^{-}$), and, unless the solution contains free acid (which will remove the hydroxide ions), the iron will be partially precipitated in the form of a basic ferric salt. Moreover, upon boiling an aqueous solution of ferric - sulphate, owing to the increase of hydrolysis with the temperature, there results a partial precipitation of the iron, again as a basic ferric salt (e.g. $\left.\mathrm{Fe}_{2}\left(\mathrm{SO}_{4}\right)_{3}+2 \mathrm{H}^{+} \mathrm{OH}^{-} \leftrightarrows 2 \mathrm{Fe}(\mathrm{OH}) \mathrm{SO}_{4}+\mathrm{H}_{2} \mathrm{SO}_{4}\right)$. This action, however, is prevented by the presence of sufficient hydrochloric acid; the hydrogen-ion of the acid, by mass action, prevents the ionization of the water.

2. The complete oxidation of the iron is necessary, since ferrous-ion is not quantitatively precipitated by ammonia; in the absence of air, 
indeed, ammonium salts are capable of preventing entirely the precipitation of iron by ammonia from ferrous salt solutions. Ferric-ion, on the other hand, is completely precipitated by ammonia, even in the presence of ammonium salts.

The nitric acid oxidizes the iron according to the equation:

$$
6 \mathrm{FeSO}_{4}+2 \mathrm{HNO}_{3}+6 \mathrm{HCl}=2 \mathrm{Fe}_{2}\left(\mathrm{SO}_{4}\right)_{3}+2 \mathrm{FeCl}_{3}+2 \mathrm{NO}+4 \mathrm{H}_{2} \mathrm{O} \text {, }
$$

and the darkening of the solution is due to the union of nitric oxide with still unoxidized ferrous salt, to form unstable $\mathrm{FeSO}_{4}(\mathrm{NO})$; this, however, is soon decomposed, and the nitric oxide expelled. ( $C f$. the brown-ring test for nitric acid.)

To insure the presence of iron wholly in the ferric condition, a very small quantity of the oxidized solution should be tested on white porcelain with a drop of freshly made potassium ferricyanide solution (dissolve a cubic millimeter of the salt in $15-20 \mathrm{cc}$. of water). If the iron solution has a volume of $50 \mathrm{cc}$., and an ordinary drop a volume of $0.05 \mathrm{cc}$., then the loss of the latter would occasion an error of $0.1 \%$ in the iron (and in the sulphur) determination. In this case, therefore, we should add a couple of drops of the iron solution to I cc. of water in a clean watch glass, and use I drop of this mixture for the test ; the remainder should be washed back into the beaker containing the bulk of the solution. The error is thus reduced to $0.01 \%$, which is negligible.

Much time can often be saved by testing in this way the solution made from a little of the (unweighed) original sample, for ferrous iron; in its absence the addition of the nitric acid, and its subsequent removal by evaporation to dryness, should be omitted.

3. Upon the addition of ammonia in slight excess only, to the ferric salt solution, the resulting precipitate would be sure to contain basic salts in appreciable quantity, and the separation of the sulphate radical would be incomplete. By digesting the precipitate with a large excess of ammonium hydroxide, however, these basic compounds are more or less completely decomposed into ferric hydroxide and the corresponding ammonium salts; nevertheless, since a small quantity of the basic sulphate might escape this action, the precipitate is redissolved in hydrochloric acid and the operation repeated. In this way, the quantitative separation of the sulphate radical is assured.

4. Owing to the solvent action of alkaline liquids on glass, the filtration should be promptly carried out, and the filtrate and washings at once neutralized. The precipitate, which is of a colloidal nature, will give no trouble if it is filtered off hot, and is promptly washed with very hot water. If allowed to cool off in the mother liquid, or if washed with water that is 
too cold, it is almost sure to become slimy, and very difficult to prepare for ignition.

5. During the decomposition of the paper and the combustion of the carbon, the precipitate may be reduced in part to $\mathrm{Fe}_{3} \mathrm{O}_{4}$, and this must be oxidized back to $\mathrm{Fe}_{2} \mathrm{O}_{3}$. Therefore, during ignition, the air should have free access to the oxide; and, in order to insure a porous mass which is readily reoxidized, the filter originally used is macerated with the solution before the second precipitation.

Concerning the effect of ammonium chloride upon ferric and aluminum hydroxides during ignition, see H. W. Daudt, Jour. Ind. Eng. Chem., vol. 7, p. 847 (I9I5).

6. The foregoing method may be used for the gravimetric determination of chromium or aluminum, but at the time of filtration the solution should contain but a very slight excess of ammonia; chromium and aluminum hydroxides are appreciably soluble in strong ammonia. Owing to the tendency of aluminum, when thrown down by ammonia, to carry down and retain basic compounds, and also to the slimy nature of the precipitate so obtained, it is better in the case of this metal to add sodium thiosulphate in excess to the dilute solution, and then to boil off the sulphur dioxide, add ammonia in slight excess, and filter boiling hot. This gives a compact, easily prepared precipitate, which on ignition yields pure $\mathrm{Al}_{2} \mathrm{O}_{3}$ :

$$
\begin{gathered}
2 \mathrm{AlCl}_{3}+6 \mathrm{HOH} \leftrightarrows 2 \mathrm{Al}(\mathrm{OH})_{3}+6 \mathrm{HCl} ; \\
\text { and } 6 \mathrm{HCl}+3 \mathrm{Na}_{2} \mathrm{~S}_{2} \mathrm{O}_{3}=6 \mathrm{NaCl}+3 \mathrm{H}_{2} \mathrm{O}+3 \mathrm{SO}_{2}+3 \mathrm{~S}
\end{gathered}
$$

If it is desired by this method to determine the chromium in an alkali chromate, the latter is boiled with hydrochloric acid and alcohol, in order to reduce the chromium to the trivalent condition:

$$
\mathrm{K}_{2} \mathrm{Cr}_{2} \mathrm{O}_{7}+3 \mathrm{C}_{2} \mathrm{H}_{6} \mathrm{O}+8 \mathrm{HCl}=2 \mathrm{KCl}+{ }_{2} \mathrm{CrCl}_{3}+{ }_{3} \mathrm{C}_{2} \mathrm{H}_{4} \mathrm{O}+7 \mathrm{H}_{2} \mathrm{O} \text {. }
$$

While chromic hydroxide is decomposed by heat into water and the oxide, the latter upon ignition is oxidized to a small extent to $\mathrm{Cr}_{2}\left(\mathrm{CrO}_{4}\right)_{3}$; in the case of this metal, therefore, the oxide should be ignited in a Rose crucible, in an atmosphere of hydrogen (G. Rothaug, Zeitschrift für anorganische Chemie, vol. 84, pp. 165-r89 (1913)).

7. The hydroxides of all three metals are precipitated by sodium or potassium hydroxide, but the precipitates are always contaminated with alkali. Furthermore, aluminum and chromium hydroxides dissolve readily in caustic alkali, yielding complex anions to which the formulas $\mathrm{AlO}_{2}^{-}$and $\mathrm{CrO}_{2}^{-}$are usually ascribed, but which more likely are $\left[\mathrm{Al}(\mathrm{OH})_{4}\right]^{-}$and $\left[\mathrm{Cr}(\mathrm{OH})_{4}\right]^{-} . \quad\left(\mathrm{Cf} . \quad \mathrm{AuCl}_{3}+\mathrm{HCl}=\mathrm{H}\left[\mathrm{AuCl}_{4}\right] ;\right.$ and $\mathrm{Cr}(\mathrm{OH})_{3}+\mathrm{NaOH}$ $=\mathrm{Na}\left[\mathrm{Cr}(\mathrm{OH})_{4}\right]$.) 
The trivalent ions of iron, chromium, and aluminum form stable complexes with tartrate and citrate ions (and with certain other organic materials); so that tartrates, for example, are capable of entirely preventing the precipitation of $\mathrm{Fe}(\mathrm{OH})_{3}, \mathrm{Cr}(\mathrm{OH})_{3}$, and $\mathrm{Al}(\mathrm{OH})_{3}$ by alkali hydroxides. These and other analogous facts are often made use of in analytical chemistry.

B. Procedure for the Determination of Sulphur. Evaporate to dryness, on the steam bath, the combined filtrates and washings from the iron determination; add Io cc. of 6-normal hydrochloric acid; and again evaporate to dryness. ${ }^{1}$ To the residue add soo cc. of water, heat to boiling, and filter into a $700 \mathrm{cc}$. beaker, thoroughly washing with hot water until the filtrate and washings have a volume of $400 \mathrm{cc}$. Heat this solution to boiling, and, with stirring, quickly pour in a boiling-hot mixture made by adding $15 \mathrm{cc}$. of I-normal barium chloride solution to roo cc. of water. Digest with stirring for a few minutes at the boiling temperature; remove the flame and allow to stand for half an hour; and, after testing for complete precipitation, decant the clear liquid through a filter. Wash the precipitate by decantation, and then upon the filter, with hot water, until $5 \mathrm{cc}$. of washings give no test for chloride-ion. Dry the filter and precipitate and ignite to constant weight, as in the preceding determination. Report the percentage of $\mathrm{SO}_{4}$ in the sample.

Notes. - I. Barium sulphate, to a greater degree than most precipitates, has the property of carrying down various soluble salts which may be present, and these cannot be removed by simply washing the precipitate. This is especially true of the salts of trivalent metals (as iron, chromium, etc.), and of nitrates and chlorates. If, therefore, nitric acid has been used to oxidize the iron, it must be completely removed by evaporation to dryness with an excess of hydrochloric acid.

Iron is always present in the sulphate precipitated from solutions containing ferric salts; the precipitate evolves sulphuric acid on heating, and

${ }^{1}$ In case the original sample contained no ferrous iron, and the addition of nitric acid was omitted, it is sufficient only to evaporate the neutralized filtrates and washings to about $300 \mathrm{cc}$. This liquid is then transferred to the beaker, diluted to about $400 \mathrm{cc}$., and treated further as described in the procedure. 
yields low results in spite of its iron content. Pure barium sulphate itself is not decomposed at a red heat, but suffers loss, probably of sulphur trioxide, at a temperature above $900^{\circ}$.

The solubility of barium sulphate is about 2.5 parts per million of water, but it is somewhat more soluble in hydrochloric acid, even very dilute; in many salt solutions it is still more soluble.

2. In the precipitation of sulphuric acid with barium chloride, the solution should contain only salts of the alkali metals and ammonium, and it should be free from nitrates and chlorates. Even alkali salts and barium chloride are carried down to some extent by barium sulphate, more or less in proportion to their concentration, and consequently the solution should be dilute. Since, further, the solubility of barium sulphate, as well as the amount of barium chloride carried down, increases with the concentration of the hydrochloric acid present, the quantity of the latter should be reduced to a minimum; some, however, must be present, since otherwise the precipitate would be very fine grained, and therefore difficult to filter.

Barium sulphate carries down quantities of chlorine varying from traces to as much as $\mathrm{r} \%$, depending upon the conditions, and these should therefore be very carefully regulated. For a quantity of sulphate corresponding to $\mathrm{I}-2 \mathrm{~g}$. of $\mathrm{BaSO}_{4}$, the latter should be precipitated from a solution which has been diluted to about $400 \mathrm{cc}$., and which should contain I.5 cc. of free 6-normal hydrochloric acid. This solution should be boiling hot, and, for each gram of barium sulphate, Io cc. of I-normal barium chloride solution diluted to roo cc., and boiling hot, should be poured in, all at once, with constant stirring. In this way exact results can be obtained, but only owing to a compensation of errors: although a very small quantity of barium sulphate remains dissolved in the acidified salt solution, an approximately equal weight of barium chloride is contained in the ignited precipitate.

If precipitated as described, and with the use of a good filter, the precipitate shows no tendency to pass through the pores of the paper; it is even possible to use with success a Munroe crucible in this determination.

3. The barium sulphate may be partially reduced to the sulphide upon ignition with a paper filter; in order to prevent this as far as possible, the crucible should not be heated above dull redness until the carbon has been consumed. Ignition in the presence of air will then suffice to oxidize the sulphide back to sulphate. 
THE DETERMINATION OF SULPHUR IN A SULPHIDE ORE

Method. The ore is heated with strong nitric acid and potassium chlorate, in order to oxidize the sulphur to sulphuric acid. After removing the nitric acid and chlorate, as well as the iron, lead, etc., the sulphuric acid is precipitated with barium chloride, and weighed as barium sulphate.

Procedure. Treat $0.25^{-0.50} \mathrm{~g}$. samples of the finely pulverized ore (depending upon the sulphur content) in $250 \mathrm{cc}$. Erlenmeyer flasks with ro cc. of nitric acid (sp. gr. 1.42), and heat very gently until the red fumes have somewhat abated. Then increase the heat, and add to the quietly boiling liquid potassium chlorate, from time to time, in o.I g. portions, until any free sulphur which has separated is entirely oxidized and dissolved ; finally add $0.5 \mathrm{~g}$. of solid sodium chloride and evaporate the solution to dryness. After cooling, cautiously add Io cc. of hydrochloric acid (sp. gr. I.I9), heat gently until solution is as complete as possible, and evaporate to dryness. Take up in $5 \mathrm{cc}$. of strong hydrochloric acid, heat to boiling, and dilute with roo cc. of cold water. To the cold solution add three drops of methyl orange, and ammonia to alkaline reaction; then add 5 cc. more of ammonia and ro cc. of ammonium carbonate solution. Heat to boiling, allow the precipitate to settle in the hot liquid, and filter while still hot, washing thoroughly with hot water, and receiving the filtrate and washings in a 700-cc. beaker. Neutralize the filtrate with hydrochloric acid, and add I.5 cc. of the 6-normal acid in excess. Dilute the solution to $400 \mathrm{cc}$, heat to boiling, and add with stirring a boiling-hot mixture of Io cc. of I-normal barium chloride solution and Ioo cc. of water. Allow the mixture to stand for half an hour, test for complete precipitation, and finish the determination as described in the previous Procedure. Report the percentage of sulphur in the sample.

Notes. - I. Barium sulphate, if present in the ore, remains practically unaffected by the above acid treatment. If it is desired to determine the 
total sulphur in ores containing barium, the hydrochloric acid solution, after the removal of nitrates and chlorates and dilution with roo cc. of water, may be treated with $\mathbf{5} \mathrm{g}$. of solid ammonium chloride (to hold any lead in solution), heated to boiling, and filtered from the insoluble residue. The filter containing the latter is destroyed by ignition in a platinum crucible, and the residue fused with an excess of sodium carbonate. The fusion is extracted with hot water and the residue washed with sodium carbonate solution; the filtrate and washings, which contain sodium sulphate, are added to the hydrochloric acid filtrate containing the bulk of the sulphur. The united filtrates are then treated with ammonia and ammonium carbonate, as described above.

2. The small amount of sodium chloride is added before the first evaporation in order to prevent the possible loss of any free sulphuric acid which might be present, - as, for example, in the analysis of pyrites. The potassium chlorate added would, however, probably be sufficient in most cases to accomplish this result. In the analysis of pyrites, which contains a very high percentage of sulphur, samples should be used of only $0.25 \mathrm{~g}$. Otherwise the procedure is the same.

3. Upon adding ammonia in excess to the solution and heating to boiling, there is practically no danger of losing sulphur in the form of basic ferric sulphate. (In this connection see Note 3 of the procedure for iron.) The ammonium carbonate is added in order to remove any lead which may be present, as the carbonate, and thus prevent the loss of sulphur, as $\mathrm{PbSO}_{4}$, before the precipitation with barium chloride.

4. In neutralizing a solution with the use of methyl orange, the solution should be cold, since otherwise the methyl orange is not a satisfactory indicator.

5. The student should be sure to read the notes on the determination of sulphur in iron sulphate. 


\section{THE DETERMINATION OF POTASH IN SOLUBLE SALTS}

The sample may be a pure salt, a soluble industrial product, or an artificial mixture of potassium chloride and sodium carbonate.

Principle. The determination of potassium by this method depends upon the insolubility of potassium perchlorate, and the solubility of sodium and certain other perchlorates in $96 \%$ alcohol. This is not a precipitation method, but one of extraction. If heavy metals are present, they are first removed.

Procedure. Weigh out samples sufficient to contain about 0.25 g. of $\mathrm{K}_{2} \mathrm{O}$, into $50 \mathrm{cc}$. beakers, and treat each as follows: Warm the sample with $25 \mathrm{cc}$. of water, and, if sulphates are absent, filter into a Ioo cc. porcelain dish; if, however, sulphates are present, acidify with 6-normal hydrochloric acid, stir, treat the hot acid liquid with barium chloride solution in slight excess, filter into a roo cc. porcelain dish, evaporate the filtrate to dryness on the steam bath, and warm the residue with $5^{-20} \mathrm{cc}$. of water, with stirring. Add to the solution sufficient perchloric acid to contain $\mathrm{r} .7$ times the sample's weight of $\mathrm{HClO}_{4}$ (see Note 2), and evaporate to a sirupy consistency. Add ${ }_{5}$ cc. of hot water and $2 \mathrm{cc}$. of perchloric acid, and again evaporate. Once more add ${ }_{5} \mathrm{cc}$. of hot water, and evaporate until heavy fumes of perchloric acid appear.

Allow the mixture to cool thoroughly, add $20 \mathrm{cc}$. alcohol containing $0.2 \%$ by weight of $\mathrm{HClO}_{4},{ }^{1}$ and stir for some time, keeping the salt as coarsely granular as possible. Let settle, decant the liquid through a weighed Gooch crucible $^{2}$ (containing a mat moistened with the wash liquid), and to the residue add a second 20-cc. portion of the wash liquid. Stir, let settle, again decant, and then drive off the remaining alcohol on the steam

1 Made by mixing $1.7 \mathrm{cc}$. of the $60 \%$ acid, or $4.4 \mathrm{cc}$. of the $30 \%$ acid, with one liter of $96 \%$ alcohol.

2 It is better to use a Munroe crucible, with a filter of platinum sponge. The crucible itself may be of gold, to save expense. 
bath. Dissolve the residue in 15 cc. of hot water, add a few drops of perchloric acid, and evaporate to heavy fumes. Cool, add I cc. of the wash liquid, decant, and test a few drops of the washings for complete extraction. (The extraction with the alcoholic liquid must be continued until a few drops of the filtrate leave no residue when evaporated to dryness on platinum foil.) Finally, cool, add I cc. of the wash liquid, and sweep the salt into the Gooch crucible with a policeman, washing at last with a very little pure $96 \%$ alcohol. Dry the salt for half an hour at $130^{\circ}$, and weigh.

Report the percentage of $\mathrm{K}_{2} \mathrm{O}$ in the sample.

Notes. - I. This method was proposed in $183 \mathrm{I}$ by Sérullas, but, owing to some mistaken ideas concerning the properties of perchloric acid, the proposition did not receive the attention it deserved. Perchloric acid solutions of satisfactory grade can now be obtained in the market, they can be kept indefinitely in glass-stoppered bottles, and the method rivals in results the chloroplatinic acid process; and this at a greatly reduced cost.

2. The specific gravities of perchloric acid solutions are as follows: $70 \% \mathrm{HClO}_{4}, \mathrm{I} .67 ; 60 \% \mathrm{HClO}_{4}, \mathrm{I} .54 ; 50 \% \mathrm{HClO}_{4}, \mathrm{I} .4 \mathrm{I} ; 30 \% \mathrm{HClO}_{4}$, I.20; $20 \% \mathrm{HClO}_{4}$, I.I2. The strength of a solution of the pure acid may easily be determined by the dilution of a known amount and titration with sodium hydroxide, with phenolphthalein as indicator.

3. In order to obtain the potassium as pure $\mathrm{KClO}_{4}$ by this method, it is essential that no strong acids be present, other than perchloric acid, which yield salts insoluble in alcohol. Sodium chloride and sulphate are such salts, and it is therefore necessary to remove chlorides and sulphates before the treatment with alcohol. $\mathrm{HCl}$ may be removed by repeatedly evaporating the aqueous solution with the less volatile $\mathrm{HClO}_{4}$; but $\mathrm{H}_{2} \mathrm{SO}_{4}$ is less volatile than $\mathrm{HClO}_{4}$, and cannot be expelled in this way. Before the first evaporation, therefore, the latter should be precipitateci from the hot acid solution by means of $\mathrm{BaCl}_{2}$ in slight excess. Phosphates, though often insoluble in alcohol, need not be removed; but, in their presence, a larger excess of $\mathrm{HClO}_{4}$ should be used, to insure their complete removal by the wash liquid, as $\mathrm{H}_{3} \mathrm{PO}_{4}$. (See Note 5 of this procedure, and also Note 6 under the determination of calcium.)

4. Since $\mathrm{NH}_{4} \mathrm{ClO}_{4}$ is only sparingly soluble in alcohol, ammonium salts should be carefully expelled by gentle ignition, before the treatment with $\mathrm{HClO}_{4}$. Moderate amounts of barium, calcium, and magnesium do not interfere with the procedure; their perchlorates are soluble in alcohol. 
5. The perchlorate mixture is extracted with alcohol containing a small amount of $\mathrm{HClO}_{4}$ because, owing to the common ion effect, the solubility of $\mathrm{KClO}_{4}$ is less in it than in pure alcohol; the two solubilities are about $4 \mathrm{mg}$. and $16 \mathrm{mg}$. per $100 \mathrm{cc}$., respectively. The solubility of $\mathrm{KClO}_{4}$ is still less in the presence of sodium, and other soluble perchlorates; i.e. in the first portions of the alcoholic extract. Alkali and alkali-earth phosphates are decomposed by perchloric acid and the $\mathrm{H}_{3} \mathrm{PO}_{4}$ dissolves in the acid-alcoholic liquid; in the presence of phosphates, therefore, perchloric acid should be present in considerable excess.

6. If a known weight of $\mathrm{NaCl}-\mathrm{KCl}$ mixture, obtained for example in a silicate analysis, is converted into a mixture of the perchlorates, and the $\mathrm{KClO}_{4}$ isolated and weighed, the method yields both the $\mathrm{K}_{2} \mathrm{O}$ and $\mathrm{Na}_{2} \mathrm{O}$ contents of the original sample. (Cf. Part V, Problem 29.)

7. In order to prevent the loss of $\mathrm{KClO}_{4}$, in the separation of sodium and potassium, it has been suggested to extract the perchlorate mixture with an alcoholic liquid which has previously been saturated with $\mathrm{KClO}_{4}$. This procedure, however, is apt to lead to high results, owing to the precipitation of small amounts of potassium from the wash liquid by the $\mathrm{NaClO}_{4}$ entering into solution; it is therefore not to be recommended. In order to obtain exact results, it suffices to avoid the use of unnecessary quantities of the wash liquid. (See, however, G. P. Baxter and M. Kobayashi, Jour. Amer. Chem. Soc., Vol. 42, pp. 735 and 2046 (1920).) 
THE DETERMINATION OF CARBON DIOXIDE IN LIMESTONE

Method. The weighed carbonate is placed in an apparatus which contains acid in a separate compartment; the whole apparatus is then weighed. After this the acid is run in upon the carbonate, and the carbon dioxide set free is removed from the apparatus through a tube filled with calcium chloride, which prevents the escape of moisture from the apparatus. Finally, the apparatus is weighed again, and the loss in weight indicates the quantity of carbon dioxide in the sample.

Many different forms of apparatus have been devised for this purpose. The one shown in the accompanying figure is an improved form of the so-called alkalimeter of Mohr. It consists of a small, wide-mouthed, flat-bottomed flask $F$, which has a ground-glass connection with the tubes $A$ and $B$, which are for acid and calcium chloride. The ground-glass joints are lubricated with a mixture of vaseline and beeswax, or other suitable substance.

Procedure. Thoroughly clean the apparatus, allow it to drain, and finally dry it by gently heating the flask while drawing a current of dry air through it, by means of an aspirator. During aspiration the tubes $C$ and $D$ should be connected with $c$ and $d$, as shown in the figure, to prevent the entrance of moisture into the apparatus. After drying the apparatus, cover the bottom of $B$ with a loose wad of cotton, and, through a cylinder of glazed paper inserted in the neck of $B$, introduce small pieces of calcium chloride until the tube is three fourths full; upon removing the paper funnel, be careful to keep the upper walls of the tube free from the salt. Place another wad of cotton above the calcium chloride, insert the stopper, and close the tube at $d$ by slipping over it a short rubber tube stoppered with a piece of glass rod. ${ }^{1}$

${ }^{1}$ The tube must be kept closed when not in use, to prevent the gradual absorption of moisture from the air. Each of the ordinary calcium chloride tubes previously mentioned is filled in the same way about two thirds full, but in this case a 


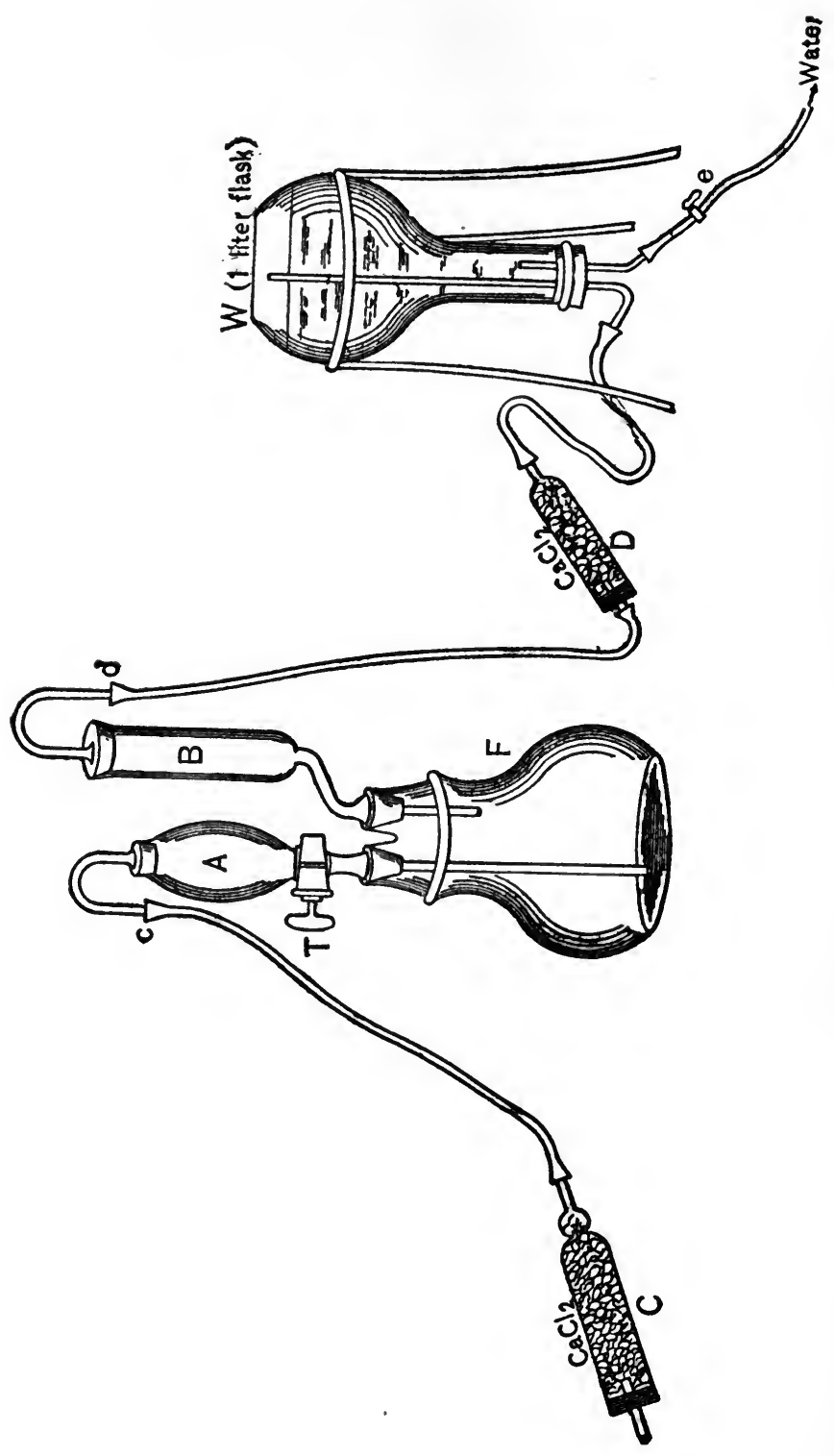


When all is ready, weigh out into the flask about $1.5 \mathrm{~g}$. of the finely powdered substance, and add $3-4$ cc. of water. Close the stopcock $T$ and fill the tube $A$ about three fourths full of hydrochloric acid (I volume of 6-normal acid to I.5 volumes of water) by means of a small funnel. The whole apparatus, with the tubes open at $c$ and $d$, is now accurately weighed; the two calcium chloride tubes are connected at $c$ and $d$; and the stopcock $T$ is slightly opened so that the acid from $A$ slowly drops into the flask. After a quiet evolution of carbon dioxide has begun, leave the apparatus to itself for about half an hour, until all of the acid has entered the flask.

In order wholly to remove the carbon dioxide from the apparatus, connect the calcium chloride tube $D$ with the aspirator $W$, as shown in the figure (this bottle is of course much larger than the figure would indicate), and, by means of a screw clamp, regulate the flow of water through $e$ so that air will be drawn through the flask $F$ at the rate of only 2 or 3 bubbles per second. Then heat $F$ gently, by means of a small flame, until the acid just begins to boil; at once remove the flame, and continue to aspirate air through the apparatus until it is cold.

Stopper the tubes at $c$ and $d$, wipe the apparatus with a clean dry towel, and allow it to stand for one half hour near the balance. Finally, remove the stoppers from $c$ and $d$, and weigh the apparatus. Report the percentage of $\mathrm{CO}_{2}$ found.

Notes. - I. This method yields excellent results in the estimation of large amounts of carbonic acid, in limestones and baking powders, but it is unreliable for the determination of small quantities.

2. Since baking powders are decomposed by water, they should be kept dry until after the apparatus has been weighed; and since their efficiency as leaveners depends upon the volume of gas liberated under the conditions of actual usage, water should be placed in the tube $A$, instead of acid.

softened cork stopper, pierced by a short piece of glass tubing with rounded ends, is introduced and shoved far into the tube with the help of a stirring rod, leaving the outer 2 or $3 \mathrm{~mm}$. empty. This space in the tube is filled with molten sealingwax, so that an air-tight connection is made. These tubes are also closed, when not in use, by glass rods within rubber tubing. 
Otherwise the procedure is the same. The loss in weight is then a measure of the available carbon dioxide of the sample.

3. Carbon dioxide is readily displaced from the apparatus by the method described, but in order to insure its complete removal at least a liter of air should be drawn through the apparatus. The small tube from $A$ should project well below the surface of the liquid, for in order to remove carbon dioxide efficiently from the solution, the air must be made to bubble through the liquid.

4. Since commercial calcium chloride is apt to contain free lime, it should for the best results be treated with carbon dioxide before the determination is made. For this purpose a current of the dry gas is passed through the apparatus for a minute or two, the tubes at $c$ and $d$ are closed, and the apparatus allowed to stand overnight. The carbon dioxide is then removed by aspirating dry air through the apparatus for about 20 minutes, after which the sample may be placed in the flask.

5. The most serious objection to this method is the fact that, owing to the size and weight of the apparatus, there is likely to be an appreciable error in the difference between the two weights. This danger, however, can be largely overcome if a similar piece of apparatus is available as a tare. (See Part I, p. 21.) 


\section{THE DETERMINATION OF CALCIUM AND MAGNESIUM OXIDES IN LIMESTONE}

Method. The hydrochloric acid extract of the limestone ${ }^{1}$ is freed from dissolved silica; treated with bromine water and ammonia, to remove iron, aluminum, manganese, etc. ; and from the filtrate the calcium is separated by double precipitation with ammonium oxalate, and the precipitate ignited to the oxide. The united filtrates from the calcium oxalate are treated with sodium phosphate and ammonia, and the precipitate of magnesium ammonium phosphate is ignited to magnesium pyro-phosphate.

$A$. Procedure for the Determination of Calcium. Weigh out into two casseroles $0.5-0.6 \mathrm{~g}$. samples of the finely ground rock, and treat each as follows: Cautiously moisten the powder with 5 cc. of water, cover the casserole, add Io cc. of 6-normal hydrochloric acid in small portions, and evaporate to dryness on the steam bath. Then add 5 cc. of water and Io cc. of the hydrochloric acid, evaporate to dryness, and continue to heat the dry residue on the steam bath for 30-45 minutes. Now add $25 \mathrm{cc}$. of water and ro cc. of the 6-normal acid, and heat for 10 minutes; filter off the residue, washing it with $5 \mathrm{cc}$. of dilute hydrochloric acid and then with hot water until free from chlorine. (See Note $I$ in regard to the insoluble residue.)

Add to the filtrate and washings bromine water, to a distinctly yellow tinge; then boil, and add ammonia until its odor just persists in the solution. Heat to boiling, and filter at once, washing with hot water, and allowing the washings to run into the filtrate. Now, with the precipitation beaker under the funnel, pour over the filter $25 \mathrm{cc}$. of hot hydrochloric acid (5 cc. of 6-normal acid, diluted to $25 \mathrm{cc}$.) ; do not bother if a slight residue remains, but wash the filter thoroughly with hot water, and reprecipitate the iron, etc., from the filtrate and washings with bromine water and ammonia, as already described. Trans- 
fer the precipitate to the filter previously used, and wash thoroughly with hot water, adding the filtrate and washings to those obtained in the first operation. (Concerning the precipitate, see Note I.)

Evaporate the combined filtrates and washings to about 200 cc.; if necessary, add ammonia until its odor is plainly perceptible, and then to the boiling-hot liquid add ammonium oxalate solution gradually with stirring, as long as a precipitate continues to form, finally adding an excess of ro cc. Boil with stirring, for a short time, set aside for 30 minutes, and filter as usual; washing the precipitate twice by decantation with hot water containing a very little ammonium oxalate and ammonia. (Add to the filtrate and washings $5 \mathrm{cc}$. of ammonium oxalate solution, let stand for Io-r 5 minutes, and, if no precipitate forms, acidify with hydrochloric acid and reserve for Procedure $B$.)

Redissolve the oxalate precipitate by pouring warm 3-normal hydrochloric acid slowly over the filter and receiving it in the beaker containing the bulk of the material; wash the filter with water, and then with very dilute ammonia. Dilute the filtrate and washings to $200-250 \mathrm{cc}$; heat to boiling; add I-2 cc. of ammonium oxalate solution, and ammonia to a persistent odor; boil for a minute or two, with stirring, and allow to stand as before. Filter through the filter previously used, transfer the precipitate to this filter, and wash thoroughly with hot water containing a few drops of ammonium oxalate solution and also of ammonia. (The filtrate and washings should be acidified and added to those from the first precipitation. If not already started, the evaporation of this liquid should now be begun.)

Gently ignite the dried precipitate and filter, until the latter is consumed; then increase the flame to the full, and ignite for 40-50 minutes. Finally heat the crucible for 5 minutes over the blast lamp, cool in the desiccator, and weigh; repeat this treatment to constant weight (see Note 7). Report the percentage of $\mathrm{CaO}$ found. 
Notes. - 1. The chief component of limestone, calcium carbonate, is readily attacked by hydrochloric acid, as also are some of the other components; but few limestones are so pure as to dissolve completely in hydrochloric acid. The residue may contain quartz, silicates, pyrites, or other refractory materials, and carbonaceous matter may also be present. Furthermore, the insoluble silicates of the residue are apt to contain some calcium and magnesium. The thorough analysis of a limestone necessitates the use of an elaborate system of procedures and the determination of numerous substances, but for many technical purposes the analysis may be confined to the determination of the insoluble matter and silica, of the oxides of iron and aluminum (including small quantities of the oxides of titanium, manganese, phosphorus, etc.), of calcium oxide, and of magnesium oxide.

Though its nature and amount depend somewhat upon the time of action and concentration of the acid used, the insoluble residue, if ignited and weighed, would give a fair approximation of the insoluble matter and silica of the limestone; and the ignited ammonia precipitate would roughly approximate the summation of the oxides of iron, aluminum, etc., in the soluble portion. Substances, however, such as hydrous silicates, pyrites, and carbonaceous matter in the insoluble residue, and ferrous and manganous oxides (originally present as carbonates) in the soluble portion, would not be correctly indicated by this method.

For a description of the complete analysis of limestones, the student is referred to Bulletin No. 700 of the United States Geological Survey, by W. F. Hillebrand.

2. The silicates present in the limestone are subject to partial decomposition by the acid, and the silicic acid must be converted into insoluble silica by evaporation and heating. The residue is washed first with dilute acid to prevent the separation on the filter of basic salts of iron, aluminum, etc., owing to hydrolytic action.

3. The bromine water is added to oxidize ferrous iron, and also manganese, which then precipitates as $\mathrm{MnO}(\mathrm{OH})_{2}$. This precipitate should be filtered off at once, since the alkaline solution absorbs carbon dioxide from the air, with the consequent precipitation of a little calcium as the carbonate. Owing to this fact, and also because the precipitated hydroxides tend to carry down the hydroxides of calcium and magnesium, the precipitate is redissolved and again precipitated, to free it from these metals.

4. In the separation of calcium from magnesium by means of ammonium oxalate, the calcium oxalate tends to carry down some magnesium oxalate, probably as a double salt; this must be removed by redissolving the precipitate and again throwing down the calcium from the solution containing only this small quantity of magnesium. If the quantity of magnesium 
originally present in the limestone is relatively very small, the calcium can be separated in one operation, from a rather dilute solution, by the use of ammonium oxalate in excess of the quantity required to convert both the magnesium and the calcium into oxalates. (In this connection, see T. W. Richards, C. T. McCaffrey, and H. Bisbee; Zeitschrift für anorganische Chemie, vol. 28, p. 7 I (rgor).)

5. A little ammonium oxalate is added in the second precipitation, because an excess of the reagent reduces the solubility of calcium oxalate, and also tends to hold magnesium in solution as a double ammonium magnesium oxalate, which is soluble. For the first reason, the precipitate is washed, not with pure water, but with water containing ammonium oxalate and ammonia. These substances are volatilized in the ignition.

6. Calcium oxalate, though almost insoluble in water $(5.6 \mathrm{mg}$. of the anhydrous salt per liter of water), and practically insoluble in acetic acid, is readily dissolved by hydrochloric acid. This behavior is due to the fact that oxalic acid lies about halfway in strength between acetic and hydrochloric acids. In acetic acid solution, the hydrogen-ion concentration is too low to appreciably diminish the concentration of $\mathrm{C}_{2} \mathrm{O}_{4}{ }^{--}$ions, and hence no solvent action takes place. In hydrogen chloride solution, however, the high hydrogen-ion concentration causes the calcium oxalate to dissolve according to the following scheme:

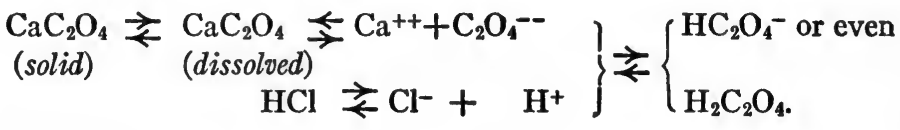

Upon the addition of a base to this solution, hydroxide-ion removes hydrogen-ion to form water, $\mathrm{C}_{2} \mathrm{O}_{4}-$ is regenerated, and this unites with $\mathrm{Ca}^{++}$to form $\mathrm{CaC}_{2} \mathrm{O}_{4}$, which precipitates.

7. Upon ignition, calcium oxalate becomes anhydrous slightly above $180^{\circ}$, and at low redness it is decomposed into calcium carbonate and carbon monoxide. Strong ignition converts the carbonate into the oxide; in a platinum crucible, this conversion may be carried to completion over a large Meker burner. In any case, however, after the filter is consumed, a good muffle furnace, or an electric crucible furnace, is preferable for the ignition. This is especially true with porcelain crucibles.

Since calcium oxide (quicklime) absorbs moisture and carbon dioxide from the air, it should be weighed as rapidly as possible.

8. By burning off the filter and then evaporating with 2-3 cc. of 6-normal sulphuric acid, the calcium oxalate may be converted into sulphate, and this may be heated to constant weight. While this procedure is preferred 
by some analysts, it is nevertheless disadvantageous, since it involves danger of loss by spattering. Moreover, calcium sulphate is more readily decomposed upon ignition than barium sulphate, and there is some danger of loss on this account.

B. Procedure for the Determination of Magnesium. Evaporate the united filtrates and washings from $A$ on the steam bath to incipient crystallization, add I-2 cc. of 6-normal hydrochloric acid, and then with stirring add water cautiously, a little at a time, until the salts redissolve. (If the acid solution contains solid matter at this point, it should be filtered.) Carefully add ammonia just to alkaline reaction (methyl orange); then sodium ammonium phosphate solution, very slowly with stirring, as long as precipitation continues, and then Io cc. more. Finally add 6-normal ammonia, one volume for each three of the solution, stir vigorously for so minutes, and allow the mixture to stand for at least 6 hours, - preferably overnight.

Filter off the precipitate and wash it with $2.5 \%$ ammonia (I volume of 6-normal ammonia to 3 of water); then redissolve the precipitate by slowly pouring over the filter Io cc. of warm 2-normal hydrochloric acid and receiving the acid in the precipitation beaker. Wash the filter four times with Io cc. portions of hot water. Add to the filtrate and washings I-2 cc. of sodium ammonium phosphate solution, and then, with stirring, add from a pipette aqueous ammonia drop by drop until the odor of ammonia persists in the liquid. Stir for 2 minutes, add to the mixture one third its volume of 6-normal ammonia and allow to stand for 6 hours.

Filter as usual, transfer the precipitate to the filter by means of $2.5 \%$ ammonia, and continue to wash with this liquid until 5 cc. of washings give no opalescence with nitric acid and silver nitrate.

With free access of air, ignite the dry precipitate and filter, increasing the heat only gradually, and not above a dull red, until the paper is consumed and the precipitate is white. Fi- 
nally, ignite to constant weight over the blast lamp or in a muffle furnace. Report the percentage of $\mathrm{MgO}$ found.

Notes. - I. When washed with pure water, magnesium ammonium phosphate is hydrolyzed according to the equation,

\section{$\mathrm{MgNH}_{4} \mathrm{PO}_{4}+\mathrm{H}_{2} \mathrm{O} \rightarrow \mathrm{MgHPO}_{4}+\mathrm{NH}_{4} \mathrm{OH}$.}

The precipitate thereby loses its crystalline form, and is sure to give a cloudy filtrate. Ammonium hydroxide prevents this action by mass action, and for this reason the precipitation mixture is made to contain $2.5 \%$ of ammonia, and the washing is performed with ammonia of this concentration.

2. In the presence of ammunium salts in large quantity, as in the first precipitation, the precipitate is only partially crystalline; it is apt to contain the mixed phosphate, $\mathrm{Mg}\left[\left(\mathrm{NH}_{4}\right)_{2} \mathrm{PO}_{4}\right]_{2}$, which upon ignition leaves magnesium metaphosphate, $\mathrm{Mg}\left(\mathrm{PO}_{3}\right)_{2}$; and, if produced with a too rapid addition of ammonia, it may also contain some tri-magnesium phosphate, $\mathrm{Mg}_{3}\left(\mathrm{PO}_{4}\right)_{2}$, which upon ignition remains unchanged. This precipitate, therefore, should be purified by solution in a little hydrochloric acid, addition of a small quantity of sodium ammonium phosphate, and reprecipitation from this solution (which contains very little ammonium salt) by the gradual addition of ammonia.

3. Upon ignition, the precipitate is converted into magnesium pyrophosphate, with the evolution of ammonia and steam:

$$
2 \mathrm{NH}_{4} \mathrm{MgPO}_{4} \text {. } 6 \mathrm{H}_{2} \mathrm{O}=\mathrm{Mg}_{2} \mathrm{P}_{2} \mathrm{O}_{7}+2 \mathrm{NH}_{3}+\mathrm{I}_{3} \mathrm{H}_{2} \mathrm{O} \text {. }
$$

The precautions detailed in Part I should be carefully observed in igniting this precipitate. The phosphate is subject to partial reduction by the ammonia or by the filter, and also, if too soon heated very strongly, the precipitate becomes glazed over and it is then impossible to remove the carbon by further heating. The precipitate is much more readily ignited to whiteness in platinum than in porcelain; but in case platinum is used, especial care should be taken to provide a plentiful supply of air, to prevent reduction accompanied by injury to the crucible.

The most satisfactory procedure is to filter off the magnesium ammonium phosphate in a Munroe crucible of platinum (with a platinum sponge filter), in which the precipitate can be ignited without danger of loss. If a good muffle furnace (preferably electric, in which the air is pure) is available, a Gooch crucible of porcelain may be used. 


\section{THE DETERMINATION OF PHOSPHORIC ANHYDRIDE IN PHOSPHATE ROCK}

Method. The finely ground mineral is heated with nitric acid, the mixture evaporated to dryness, and the residue extracted with hot nitric acid; the solution is then filtered from the insoluble siliceous material. The filtrate is made almost neutral with ammonia, and is treated with a solution of ammonium molybdate, in excess, to separate the phosphoric acid from calcium, iron, aluminum, etc. The precipitated ammonium phospho-molybdate is washed with acidified ammonium nitrate solution, dissolved in ammonium hydroxide, and the phosphoric acid precipitated with magnesia mixture. The magnesium ammonium phosphate is finally ignited to magnesium pyrophosphate, which is weighed.

Procedure. Weigh out into $200 \mathrm{cc}$. casseroles two $0.18-0.20$ g. portions of the powdered mineral, and treat each as follows. Add to the sample I $_{5} \mathrm{cc}$. of 6-normal nitric acid, cover the vessel, and warm gently for a short time; then evaporate to dryness on the steam bath. Allow the dry residue to remain for 30-40 minutes on the steam bath; add $25 \mathrm{cc}$. of 6-normal nitric acid, and heat for a few moments to dissolve the soluble portion. Filter, and wash the siliceous residue and filter with small portions of hot water, receiving the filtrate and washings in' a 400cc. beaker; continue to wash as long as the water which runs through will give a turbidity with ammonia, but add to the filtrate all washings in which such a cloud is produced. Cautiously, and with stirring, add ammonia to the filtrate and washings until the liquid remains barely turbid; then nitric acid, drop by drop, until the solution clears up again. The volume of the solution at this point should be not over roo cc.

Heat the solution to about $70^{\circ}$, remove the burner, and add $80 \mathrm{cc}$. of a freshly filtered solution of ammonium molybdate. Digest for a few hours at $60-65^{\circ}$, and then decant the clear liquid through a filter, washing the yellow precipitate (kept in the 
beaker) with acid ammonium nitrate solution, ${ }^{1}$ until $5 \mathrm{cc}$. of washings give no test for calcium with ammonia and ammonium oxalate. (Before disposing of the filtrate, digest it at $65^{\circ}$ with 5-10 cc. more of the molybdate reagent. If no more of the yellow precipitate is formed, the liquid should be poured into the bottle for "Molybdate Residues.")

Place the beaker containing the yellow precipitate under the funnel, slowly pour over the filter four separate ro cc. portions of a warm $2.5 \%$ solution of ammonia, ${ }^{2}$ and carefully wash the filter five times with ro cc. portions of hot water.

To the clear solution add hydrochloric acid, drop by drop, with stirring, until the yellow cloudiness produced disappears only slowly upon stirring. To this solution add $20 \mathrm{cc}$. of magnesia mixture from a pipette, at the rate of about I drop per second, with vigorous stirring (see Note 7 ; the last ro cc. may be added somewhat faster). Let stand for I 5 minutes, add I $5_{5}$ cc. of I 5-normal ammonia, and then, after a period of 2 or 3 hours, decant the clear liquid through the original filter and transfer the precipitate to the filter by means of $2.5 \%$ ammonia water. Continue the washing with this liquid until $5 \mathrm{cc}$. of washings, after acidification with nitric acid, give no opalescence with silver nitrate solution.

Dry the precipitate and filter, and ignite with free access of air, increasing the heat only gradually, and not above a dull red, until the paper is consumed and the precipitate white. Finally, ignite to constant weight over the blast lamp or in a muffle furnace. Report the percentage of $\mathrm{P}_{2} \mathrm{O}_{5}$ found.

NOTES. - r. The dehydration and removal of any dissolved silicic acid is necessary; like tungstic acid, molybdic acid tends to form with silicic acid substances of great complexity, and these, if present in the phospho-molybdate precipitate, would be likely to interfere with its complete solubility in ammonia.

1 Made by mixing $50 \mathrm{cc}$. of 6-normal ammonium hydroxide with roo $\mathrm{cc}$. of 6-normal nitric acid, and diluting the mixture with $350 \mathrm{cc}$. of water.

${ }^{2}$ Made by mixing $25 \mathrm{cc}$. of 6-normal ammonium hydroxide with $75 \mathrm{cc}$. of hot water. 
Calcium phosphate, though almost insoluble in water, dissolves readily in nitric acid, according to the following system of equilibria :

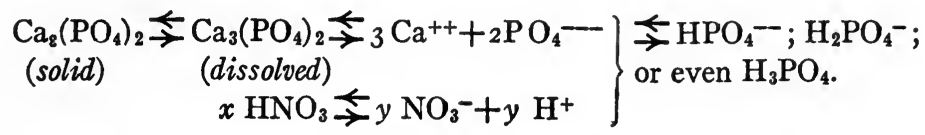

Therefore, in order to test for phosphate in the acid washings from the siliceous residue, it is only necessary to add ammonium hydroxide; hydroxideion removes hydrogen-ion, $\mathrm{PO}_{4}^{--}$is regenerated, and it unites with $\mathrm{Ca}^{++}$ which also is present to form $\mathrm{Ca}_{3}\left(\mathrm{PO}_{4}\right)_{2}$, which precipitates.

Also, later on, when it is desired to practically neutralize this solution with ammonium hydroxide, it is only necessary to add the latter to the appearance of a permanent turbidity; this indicates the almost complete removal of hydrogen-ion from the solution.

2. Nitric acid, rather than hydrochloric, is used in the precipitation of the phospho-molybdate, because molybdates are subject to a partial reduction by hydrochloric acid. Although nitric acid alone would tend to dissolve this precipitate, this action is counteracted by the presence of the ammonium nitrate which is formed upon almost neutralizing the original solution of the rock with ammonium hydroxide. Owing to this fact, in washing the precipitate free from metals with dilute nitric acid, the wash liquid also is made to contain an equivalent amount of ammonium nitrate. It should be noted, too, that the molybdate reagent contains ammonium nitrate and free nitric acid. (See the Preparation of Reagents, in the Appendix.)

3. The precipitation of the phosphoric acid as magnesium ammonium phosphate from the original solution of the rock is not possible, owing to the presence of metals such as iron, aluminum, calcium, etc., which also form phosphates insoluble in ammonia. Therefore, the phosphoric acid is previously separated from the metals, in acid solution, as ammonium phosphomolybdate.

4. While the precipitation of the phospho-molybdate should be carried out in a warm solution, the maximum temperature which can be safely employed is $65^{\circ}$; warmer solutions tend also to deposit free $\mathrm{MoO}_{3}$, which is white.

A large excess of the molybdate reagent is required to effect a complete precipitation of the phosphoric acid. Theoretically $\mathrm{I} .95 \mathrm{~g}$. of $\mathrm{MoO}_{3}$ are required to combine with the phosphate in $0.2 \mathrm{~g}$. of rock containing $40 \mathrm{per}$ cent of $\mathrm{P}_{2} \mathrm{O}_{5}$; while the quantity of the reagent actually used ( $80 \mathrm{cc}$.) contains about $5 \mathrm{~g}$. of $\mathrm{MoO}_{3}$. The presence of ammonium nitrate in the solution is also conducive to complete precipitation. These substances, by mass 
action, prevent the partial dissociation of the complex into its more soluble constituents.

5. While the composition of the yellow precipitate varies somewhat with the conditions, it nevertheless seems to correspond pretty closely to the formula $\left(\mathrm{NH}_{4}\right)_{3} \mathrm{PO}_{4}$. I2 $\mathrm{MoO}_{3} .2 \mathrm{HNO}_{3} \cdot \mathrm{H}_{2} \mathrm{O}$; at any rate, under proper analytical conditions, the ratio $\mathrm{P}_{2} \mathrm{O}_{5}: 24 \mathrm{MoO}_{3}$ holds very closely. The yellow precipitate dissolves readily in aqueous ammonia to give ammonium phosphate and ammonium molybdate; and molybdate-ion is not precipitated by magnesia mixture.

6. The precipitate obtained upon the addition of the magnesia mixture should have the formula $\mathrm{Mg}\left(\mathrm{NH}_{4}\right) \mathrm{PO}_{4} .6 \mathrm{H}_{2} \mathrm{O}$; in which case it will be wholly crystalline. The gradual addition of the reagent, with constant stirring, is conducive to this end. If magnesia mixture is added too rapidly, or without vigorous stirring, it will accumulate locally in the liquid and tend to give a precipitate contaminated with molybdic acid and magnesia. Such a precipitate will not be wholly crystalline; it should be redissolved in a small volume of hydrochloric acid, the solution treated with $1-2$ cc. of magnesia mixture, and the hot liquid slowly neutralized with $2.5 \%$ ammonia. Strong ammonia is then added, and the analysis continued as above.

Magnesia mixture is prepared by putting together in solution magnesium chloride, ammonium chloride, and ammonium hydroxide. The function of the ammonium chloride is to drive back the ionization of the ammonium hydroxide, and thus to prevent the precipitation of magnesium hydroxide, which otherwise might contaminate the precipitate.

7. If the magnesium ammonium phosphate were washed with pure water, it would be hydrolyzed according to the equation,

$$
\mathrm{MgNH}_{4} \mathrm{PO}_{4}+\mathrm{H}_{2} \mathrm{O} \rightleftarrows \mathrm{MgHPO}_{4}+\mathrm{NH}_{4} \mathrm{OH} ;
$$

it would lose its crystalline form, and be almost sure to give a cloudy filtrate. Ammonium hydroxide, however, prevents this decomposition by mass action.

8. Upon the ignition of this precipitate, steam and ammonia are evolved, and magnesium pyrophosphate is formed:

$$
2 \mathrm{NH}_{4} \mathrm{MgPO}_{4} .6 \mathrm{H}_{2} \mathrm{O}=\mathrm{Mg}_{2} \mathrm{P}_{2} \mathrm{O}_{7}+2 \mathrm{NH}_{3}+\mathrm{I}_{3} \mathrm{H}_{2} \mathrm{O} \text {. }
$$

Concerning the precautions to be observed in the ignition, see Note 3 under the determination of magnesium in limestone. 


\section{THE DETERMINATION OF SILICA IN A REFRACTORY SILICATE}

Method. The powdered sample, though not readily attacked by hydrochloric acid, is decomposed by molten sodium carbonate, with which it yields sodium silicate and other compounds readily attacked by hydrochloric acid. By evaporation and extraction with this acid, and ignition of the insoluble residue, the silica (somewhat contaminated with iron oxide and alumina) is obtained. After weighing, this is evaporated with hydrofluoric and sulphuric acids, and the sulphur trioxide expelled from the iron and aluminum sulphates by strong ignition. The weight of the impure silica less that of the ignited impurities gives the weight of silica originally in the sample.

Procedure. Weigh out into platinum crucibles two $0.75 \mathrm{~g}$. portions of the very finely ground material. Also weigh out roughly two $4 \mathrm{~g}$. portions of pure anhydrous sodium carbonate. In each case, place about $3 \mathrm{~g}$. of the flux upon the sample in the crucible, and, with the latter upon a sheet of glazed paper, mix its contents with a clean dry rod. Stir the remaining gram of flux with the rod, to remove from the latter any particles of the mixture, and then pour it upon the mixture in the crucible.

With the crucible covered, slowly heat the mixture up to the highest possibility of an ordinary burner, and then, if necessary in order to secure complete fusion, heat the mixture over a Meker burner or a blast lamp. As soon as the mass is in quiet fusion, with practically no further evolution of gas, take up the crucible in tongs applied to the upper edge, and, by means of a slow rotary motion, cause the molten substance to spread around the walls of the crucible, where it will solidify. Then, with the mass still at a dull-red heat, lower the crucible for a few seconds halfway down into cold water, but with care that no water shall enter the crucible. Allow a little time for the melt to reheat the crucible, and lower it again into the water; then set it aside to cool. The melt may then nearly always be removed 
from the inverted crucible by gentle tapping. the crucible.)

(Do not deform.

Place the melt in a $300 \mathrm{cc}$. beaker, add Ioo cc. of water and, with stirring and care to avoid loss, gradually add $30 \mathrm{cc}$. of 6normal hydrochloric acid. (Also clean the crucible and cover with acid, and wash this liquid into the beaker. In case the melt cannot be removed from the crucible, place both in the beaker and treat with water and acid as described.) Heat the beaker gently, and, if necessary, aid the disintegration of the melt by gentle pressure with the broadened end of a glass rod.

After complete disintegration, transfer the mixture to a casserole, and evaporate on the steam bath, stirring towards the end, until the residue is dry; continue to heat this on the steam bath for about an hour. Thoroughly wet the residue with $5 \mathrm{cc}$. of I2-normal hydrochloric acid, warm gently, add roo cc. of water, and heat to boiling. Filter at once, and wash with several small portions of hot 2-normal hydrochloric acid, and then with hot water until free from chlorides; receive the filtrate and washings in a casserole. Evaporate to dryness on the steam bath, heat the dry residue as before, and repeat the extraction and filtration, washing with hot acid and then with hot water, - but use a fresh filter to receive the second yield of silica.

Cautiously ignite both filters, with their contents, in a weighed platinum crucible (if only silica is to be determined, it is not necessary to know the weight of the crucible), and, after the filters are consumed, heat for half an hour at the full heat of the burner, and allow to cool. Moisten the residue with 2 or 3 drops of strong sulphuric acid, cautiously expel this, ignite to low redness, and then for half an hour over the blast lamp. Repeat the blasting for periods of 5 minutes, to constant weight.

Now add to the silica in the crucible I cc. of 6-normal sulphuric acid and 3-5 cc. of pure $48 \%$ hydrofluoric acid (Caution: this acid is capable of causing serious injury to the skin). Evaporate as far as possible on the steam bath in a well-drawing hood, adding more hydrofluoric acid, if necessary. Cautiously expel 
the sulphuric acid, heat to low redness, and finally ignite over the blast lamp, for 5-minute periods, to constant weight. The loss in weight due to this treatment represents the weight of pure silica in the sample. Report the percentage of $\mathrm{SiO}_{2}$ found.

Notes. - I. Upon fusion with sodium carbonate, silicates are decomposed with the evolution of carbon dioxide, and formation of products such as sodium silicate and aluminate, ferrous carbonate, ferric oxide, calcium and magnesium carbonates, etc.; e. g. in the case of a certain species of garnet, we may have

$$
\begin{gathered}
\stackrel{\mathrm{Mg}}{2} \mathrm{FeAl}_{2} \mathrm{Si}_{3} \mathrm{O}_{12}+7 \mathrm{Na}_{2} \mathrm{CO}_{3}=2 \mathrm{MgCO}_{3}+\mathrm{FeCO}_{3}+2 \mathrm{NaAlO}_{2} \\
+3 \mathrm{Na}_{4} \mathrm{SiO}_{4}+4 \mathrm{CO}_{2} .
\end{gathered}
$$

Owing to the evolution of gas, the heating "should be gradual and the crucible should be kept covered.

2. Unless the sample is ground very fine, the coarser particles will be sure to escape the action of the flux; the sample, therefore, should finally be ground in an agate mortar until it will wholly pass through a roo-mesh sieve.

In case a gritty residue should remain after the disintegration with hydrochloric acid, which can be detected by means of a glass rod, the sample has not been completely decomposed; the analyst should start over with a fresh sample.

3. Upon treating the fusion with considerable dilute acid, silicic acid at first tends to dissolve; upon evaporation, however, it is largely dehydrated and rendered insoluble. The use of concentrated acid instead of dilute would cause the separation of a jelly-like mass, in which metallic salts would be protected from the solvent action of the acid.

4. The silicic acid cannot be rendered wholly insoluble by a single evaporation and heating; nor are repeated evaporations and moistenings before filtration as effective in separating the silica as are alternate evaporations and filtrations. The second portion of silica obtained should be filtered off upon a fresh filter; otherwise some of the first portion is apt to reënter the solution. These facts have not yet been satisfactorily explained.

5. To free the silica as far as possible from mineral salts, the residue after evaporation should be thoroughly extracted with warm hydrochloric acid; and the solution should be diluted to a large volume to prevent the inclosure of impurities by the silica. The silica is first washed with dilute acid, to prevent the partial separation of basic salts of iron, aluminum, etc., by hydrolysis; the washing is then completed with hot water. 
6. The finely divided silica very stubbornly retains moisture, and a prolonged ignition is necessary. Even then the ignited powder should be weighed rapidly, since it tends to reabsorb moisture.

7. In spite of every care, the ignited silica is rarely pure. Upon evaporation with hydrofluoric and sulphuric acids, however, the silica is volatilized as silicon tetrafluoride and steam, and a sulphate residue is left. If the contaminating substance is an alkali salt, as sodium chloride, the residue will remain as sulphate, even at high temperatures; but certain other sulphates, as those of iron, aluminum, and titanium, evolve sulphur trioxide on ignition and leave the corresponding oxides. In the estimation of silica, the weight of impurities in the silica is always determined by weighing the residue from the hydrofluoric and sulphuric acid treatment; in order then that the impurities weighed with the silica may be as nearly as possible identical with the residue from this treatment, it is best to treat the silica before ignition with a few drops of sulphuric acid. The final residue from the hydrofluoric and sulphuric acids should also be subjected to the same temperature employed in the ignition of the silica.

8. The procedure for the determination, in the united filtrates from the silica, of the mixed oxides of iron, aluminum, etc. (in which the hydroxide precipitate would be ignited together with the residue from the hydrofluoric and sulphuric acid treatment), of calcium oxide, and of magnesium oxide, does not differ materially from that given under the determination of calcium and magnesium in limestones.

9. For a thorough study of the analysis of silicate and carbonate rocks, the student is referred to Bulletin No. 700 of the United States Geological Survey, by W. F. Hillebrand. 


\section{THE ELECTROLYTIC DETERMINATION OF COPPER}

The sample to be analyzed may be pure copper sulphate, an artificial mixture of the carbonates of copper and sodium, a copper ore, or a nickel coin. In case stationary electrodes are employed, the solution should contain not over $0.2 \mathrm{~g}$. of copper and $5 \mathrm{cc}$. of nitric acid (sp. gr. r.42), and should have a volume of roo cc.; in the case of a rotating anode, however, the solution may contain as much as $0.5 \mathrm{~g}$. of copper, and it should contain, in a volume of $100 \mathrm{cc} ., 3-5 \mathrm{cc}$. of nitric acid (sp. gr. I.42), or I cc. of sulphuric acid (sp. gr. I.84).

Method. The copper salt is decomposed by the electric current, and the copper deposited upon the cathode (negative electrode). The cathode is weighed before and after the operation, and the increase in weight indicates the quantity of copper in the sample.

The polarity of the terminals may be determined by bringing the wires, about $0.5 \mathrm{~cm}$. apart, into contact with a piece of filter paper moistened with potassium iodide solution. At the positive terminal iodine will separate and color the paper.

Cleaning the Platinum Electrodes. ${ }^{1}$ The electrodes are freed from grease by heating with dilute sodium hydroxide solution, after which they are washed with water; the cathode is then dried, allowed to cool in a desiccator, and weighed. To clean the platinum cathode after the determination, cover the deposit completely with 6-normal nitric acid, heat for at least 15 minutes, and wash.

$A$. Procedure with Stationary Electrodes. ${ }^{2}$ Dissolve a $0.5^{-}$ o.6-g. sample of copper sulphate, $\mathrm{CuSO}_{4} \cdot \mathrm{H}_{2} \mathrm{O}$, in 50 cc. of water, in a tall $150 \mathrm{cc}$. beaker; stir to complete solution, add 4 cc. of nitric acid (sp. gr., I.42), and dilute to roo cc. Immerse the electrodes in the solution and connect them in such a way that the electrode with the larger surface is made the

1. In case a silver cathode is used, see Note I2.

${ }^{2}$ If the sample is an ore, see Note Ir. 
cathode. The electrolysis, which should be carried out at a potential of I.9-2.0 volts, may be completed in the cold overnight, or in two or three hours if the temperature is kept at $70-80^{\circ}$ by means of a heated sheet of wire gauze placed a short distance below the beaker. Finally test for complete deposition by adding a little water to raise the level of the solution on the cathode; if after 30 minutes no copper is to be seen upon the fresh platinum surface, the deposition is probably complete. (Test a few cubic centimeters of the solution with sodium acetate and a drop of potassium ferrocyanide solution.) Without disconnecting the electrodes, siphon off the electrolyte while introducing distilled water, until the current ceases to pass; this is to prevent the re-solution of any of the copper by the acid liquid. Remove the cathode, wash it with water, then with alcohol, and dry it for a short time in an air bath at $85^{-} 90^{\circ}$. Allow the cathode to cool in a desiccator, and weigh.

$B$. Procedure with a Rotating Anode. ${ }^{1}$ Heat a five-cent coin with sodium hydroxide solution to free it from grease, then wash it with water, and dry at $100^{\circ}$. After cooling, weigh the coin, and dissolve it in $50 \mathrm{cc}$. of 6-normal nitric acid, in a covered casserole. Evaporate the solution to dryness on the steam bath, dissolve the residue in about soo cc. of cold water, add Io cc. of sulphuric acid (sp. gr., r.84), allow to cool, and transfer the whole to a $500 \mathrm{cc}$. measuring flask, diluting to the mark with water. Measure off one tenth of the well-mixed solution into a 50-cc. graduated flask, for transference later to the electrolytic vessel.

With the anode attached to the shaft of the rotator (which is connected by means of a mercury cup, or otherwise, with the positive terminal), and the cathode connected with the negative terminal, pour Ioo cc. of water into the electrolytic vessel and adjust the electrodes so that, when four fifths covered with water, they do not come into contact with one another, nor cause a loss of liquid, when the anode is rotated. Throw in

1 If the sample is an ore, see Note II. 
the maximum resistance of the rheostat, see that all connections are well made, and close the switch; then draw off the water, transfer the solution quantitatively to the vessel (with care that the volume of solution and washings is close to $100 \mathrm{cc}$.), start the motor, and immediately decrease the resistance of the rheostat until the ammeter registers about I ampere. In the presence of nickel, the voltage should not exceed 2.7. After about 55 minutes, test for complete deposition by adding a little water to raise the level of the solution on the cathode; if after to minutes no copper is visible on the freshly exposed platinum, the deposition is complete. When this is the case, without disconnecting the terminals, stop the rotator and draw off the solution into a large beaker, carefully pouring in water as fast as the solution flows out. ${ }^{1}$ As soon as the ammeter indicates that no current is passing, throw off the switch, remove the cathode and wash off the water with a little alcohol; dry below $100^{\circ}$, allow to cool in a desiccator, and weigh.

Notes. - I. If two platinum plates, immersed in an aqueous solution of copper sulphate, are connected by wire with the poles of a storage battery, metallic copper will be deposited upon one of the plates; under certain conditions, all of the copper will separate in the form of a compact, firmly adherent metallic film.

The process of decomposition is called electrolysis; the solution undergoing decomposition is called an electrolyte; the two poles by which the current enters and leaves the electrolyte are called electrodes. When salt solutions are electrolyzed, the positive ions (cations) move towards the negative electrode (cathode), and the negative ions (anions) towards the positive electrode (anode).

${ }^{1}$ If it is desired to determine the nickel electrolytically, evaporate this dilute solution to a volume of $25-30 \mathrm{cc}$., make slightly alkaline with ammonia, filtering off any ferric hydroxide which may be precipitated, and to the solution ( $40 \mathrm{cc}$. in volume) in the electrolytic vessel add $60 \mathrm{cc}$. of 15 -normal ammonia. Electrolyze at 3.0-3.5 volts with a rotating anode. After about an hour, test for complete deposition by adding to a few drops of the solution, neutralized with acetic acid, a drop or two of dimethyl-glyoxime solution (a red color indicates nickel). When the deposition is complete, proceed as directed in the copper determination. Finally remove the nickel from the platinum cathode by heating for at least 15 minutes with 6-normal nitric acid. 
The quantity of electricity which passes through the solution in unit time, or the speed of the current, is measured by an ammeter. The unit, called an ampere, is represented by the unvarying current which, when passed through a solution of silver nitrate, deposits metallic silver at the rate of 0.001 II $8 \mathrm{~g}$. per second. ${ }^{1}$

The electromotive force, i.e. the electrical pressure which drives the current along the circuit, is measured by the voltmeter. The unit, called a volt, is represented by the electrical pressure that produces a current of one ampere when steadily applied to a conductor whose resistance is one ohm.

The unit of resistance, called an ohm, is represented by the resistance offered to an unvarying electric current by a column of mercury I4.452 I $\mathrm{g}$. in mass, of a constant sectional area and a length of $106.3 \mathrm{~cm}$., at the temperature of melting ice.

These magnitudes are always related to one another as follows (Ohm's law) :

Quantity of electricity (amperes) $=\frac{\text { Electromotive force (volts) }}{\text { Resistance (ohms) }}$, or $i=\frac{E}{R}$.

A most satisfactory source of current for electro-analysis, in which a steady, non-fluctuating current is desired, is the lead storage cell, the E. M. F. of which is slightly over 2 volts. The voltage and other conditions demanded in the work may be provided, of course, by means of a suitable combination of such cells into a battery. In practice, the potential difference between the electrodes is regulated by means of incandescent lamps,

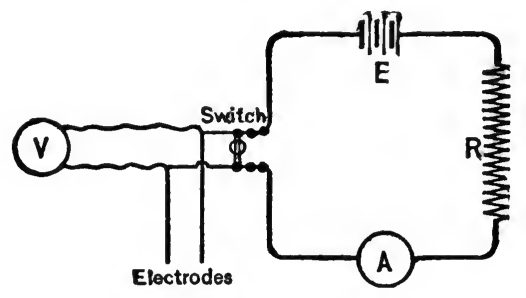

coils of wire, or other devices, which offer resistance to the flow of electricity along the circuit and convert electrical energy into heat. Any variety of

1 The quantity of a given metal deposited by a current of electricity is directly proportional to the quantity of electricity which passes through the solution; and the quantities of different metals deposited by a specific quantity of electricity are directly proportional to the chemical equivalents of the metals. These two statements are known as Faraday's laws, though these apply to nonmetallic ions as well. 
rheostat, with a suitable range in resistance, may be used in this work, but the sliding-contact coil resistances in the market are especially convenient. Ammeters and voltmeters should be such as are designed for a limited range, so that they may be read with ease and accuracy. The manner of connecting the instruments is illustrated in the figure on the preceding page.

2. The passage of an electric current of suitable voltage through the solution of an ionogen is associated with physical and chemical changes which often may be utilized in exact gravimetric analysis.

The chemical effect at the cathode is always some form of reduction. Simple metallic ions, as those of copper, tin, nickel, cobalt, cadmium, etc., travel towards the cathode, where they give up their charges and separate in the metallic condition; while the hydrogen ion here loses its positive charge and either acts directly as a reducing agent (e.g. nitric acid to ammonia) or is evolved as gaseous hydrogen.

At the anode, on the other hand, the chemical effect is always some form of oxidation. The anions of the halogen group are liberated as free chlorine, bromine, or iodine and may act as oxidizing ágents, while from solutions containing hydroxide, sulphate, or nitrate ions, oxygen separates at the anode and either acts directly as an oxidizing agent or is evolved in gaseous form. (It should be borne in mind in this connection that aqueous solutions always contain the ions of water.) Although positive ions always move towards the cathode, certain metals (e.g. lead, cobalt, nickel, and a few others) may, under specific conditions, be oxidized (possibly to complex oxy-anions) and deposited more or less completely at the anode in the form of insoluble peroxides. In fact, lead can be determined accurately in this way, as an oxide.

3. Metals, like all other substances, possess when immersed in water a characteristic solution tension, by which is understood an expansive force which seeks to drive particles of the metal into the solution; when a metal is immersed in the solution of one of its salts it will either send more of its atoms into the solution as ions, or some of its ions will be discharged from the solution on its surface as atoms. In the first case the metal will become negatively charged, and in the second case positively charged with respect to the solution; in either case equilibrium will be reached when the solution tension of the metal is exactly counterbalanced by the electrostatic charges and the osmotic pressure of the metallic ions in the solution.

Upon comparing the different elements from the standpoint of the potential difference between them and their salt solutions, at identical normal ion-concentrations, a characteristic series of values is obtained. 
In the case of solutions of normal ion-concentration, for example, some of the values are as follows:

$$
\begin{aligned}
& \mathrm{Zn}=+0.493 \quad \mathrm{Ag}=-\mathrm{r} .05 \\
& \mathrm{Cd}=+0.143 \quad \mathrm{I}=-0.80 \\
& \mathrm{Fe}=+0.067 \quad \mathrm{Br}=-\mathrm{r} .27 \\
& \mathrm{Co}=-0.045 \quad \mathrm{O}=-\mathrm{I} .50 \quad \text { (At } N . \mathrm{H}^{+} \text {-ion concentration.) } \\
& \mathrm{Ni}=-0.049 \quad \mathrm{Cl}=-\mathrm{r} .6_{3} \\
& \mathrm{H}=-0.277 \quad \mathrm{SO}_{4}=-2.18 \\
& \mathrm{Cu}=-0.606
\end{aligned}
$$

4. In an electrolysis, each electrode is soon covered with a deposit; i.e. it becomes an electrode of the material discharged. Thus, a system like that discussed above comes into existence at each electrode; and further electrolysis is opposed by the solution tensions of the discharged products. (The electrodes are said to be polarized.) In order then to decompose a salt solution continuously, a voltage at least slightly in excess of the polarization voltage must be applied; i.e. a voltage greater than the numerical difference between the individual potential differences at the two electrodes. Assuming normal ion-concentrations, the decomposition voltage of copper chloride, for example, is $-\mathrm{I} .63$ minus $-0.606=\mathrm{I} .02$ volts ; but the decomposition voltage of a solution as calculated in this manner, especially in the case of a salt of an oxyacid, frequently fails to agree with that found by experiment. The separation of gases at the electrodes is often accompanied by "overvoltages," which vary more or less markedly with the material and physical nature of the electrodes; moreover, the ion-concentration is generally unknown, and it always varies during the electrolysis. At any rate, in the case of any ionogen there always exists (under specific conditions) a definite decomposition voltage, below which the ionogen cannot be decomposed by the electric current.

In the case of an ionogen of known decomposition voltage, we should simply use a somewhat higher voltage, but if other metallic ions were present it might be impossible to completely deposit one metal without using a voltage that would start the deposition of the second metal also. While copper can readily be separated from cobalt or from nickel by electrolysis, it is not possible to separate nickel from cobalt in this way; and in general only metals whose deposition voltages differ by several tenths of a unit can be separated from each other by maintaining an intermediate voltage during the electrolysis.

The addition of certain reagents, as ammonia, potassium cyanide, ammonium oxalate, etc., to solutions containing two metals sometimes reduces the concentration of one metallic ion very much more than that of the 
other, owing to the formation of more or less stable complexes, and makes it possible to perform a separation by the "constant voltage" method that otherwise might not be possible.

5. The current strength will of course depend upon the voltage used, since, according to Ohm's law, $i=\frac{E}{R}$. In performing an electrolysis, the voltage actually available is diminished by the decomposition voltage of the electrolyte (polarization voltage); hence the current which passes is equal to the available voltage minus the decomposition voltage of the electrolyte, divided by the resistance of the circuit.

6. The quantity of metal deposited in a given time is dependent upon the strength of the current in amperes. A current of $\mathrm{I}$ ampere is capable of depositing I.II $8 \mathrm{mg}$. of silver, and, according to Faraday's law, equivalent amounts of other elements, per second. This law might be used to calculate the time necessary for the complete deposition of the metal if, under the analytical conditions, it were the only cation taking part in the electrolysis. In the neighborhood of the cathode, however, the concentration of the solution with respect to this cation gradually decreases to an infinitesimal value, and the resistance and the decomposition voltage of the solution therefore rise; finally a point is reached at which other ions begin to be discharged. Since circulation of the solution tends to maintain a uniform distribution of the ions, mechanical stirring favors the rapid deposition of those ions which have the lowest discharge voltages.

7. Unless the solution is mechanically stirred, the rate of deposition of a given metal decreases rapidly, owing to the decreasing concentration of its ions around the cathode and to the continually increasing proportion of the current which is carried by the hydrogen (or other) ions. Since a rapid circulation of the solution tends greatly to prevent the local decrease in metallic ion-concentration around the cathode, and since with improved circulation currents of much higher density may be used than would otherwise give satisfactory deposits, it is possible to greatly reduce the time necessary for a determination by performing the electrolysis with the use of a rotating electrode.

8. Owing to the reduced viscosity at higher temperatures, the resistance offered by an aqueous solution to the passage of electricity decreases with a rise in temperature, and in this way the voltage required to produce a given current may be reduced to a minimum; this may sometimes be of importance in electrolytic separations. Moreover, in case stationary electrodes are used, heating the solution during electrolysis gives rise to more or less rapid convection currents, and also increases the speed of diffusion, and these effects are equivalent to a gentle mechanical stirring. 
The solution should never be heated to the boiling point, however, since the deposit might in that case be loosened from the cathode.

9. The deposited metal tends to redissolve in the electrolyte (cf. "polarization"), and consequently the rate at which the metal is deposited must exceed that at which it redissolves. The metal is only deposited from the solution in immediate contact with the cathode, so that the greater the area of the cathode, the more metal there is available for deposition; but also the greater the rate of re-solution. Hence it follows that the current strength necessary for the satisfactory deposition of the metal is proportional to the area of the cathode.

The current strength per unit area is called the current density; a square decimeter is generally taken as the unit area. Hence a "normal current density of 2 amperes" means a current of 2 amperes per roo sq. $\mathrm{cm}$. of cathode area, or of I ampere for $50 \mathrm{sq}$. $\mathrm{cm}$. of cathode area, etc. While the tendency of a metal to redissolve fixes a lower limit for the current density to be used, a higher limit is set by the tendency of the metal to form spongy, nonadherent films when deposited too rapidly.

It is highly important for accurate work to deposit the metal in the form of a compact film which can easily be washed and weighed without loss. The condition of the deposit depends not only upon the current density used, but also upon the concentration of the metallic ions in the solution, the amount of free acid and other substances present, the temperature, etc. The best conditions for specific cases have been determined by repeated experiments.

Io. Concerning the effect upon the nature of the deposit of the products that accumulate in the solution during an electrolysis, that are purposely added to the solution, or which were originally present in the sample, it may be stated that a very marked influence is often exerted by certain acids, bases, and other substances. A solution of copper sulphate, if electrolyzed without the addition of another substance, is almost sure to give a reddish brown, non-adherent deposit of spongy copper; the addition of a little sulphuric acid gives rise to a much more compact deposit, while the addition of nitric acid leads to a still better deposit of bright red firmly adherent metal. A small quantity of urea, in addition to either acid, appears to favor still more the formation of a satisfactory deposit. On the other hand, high current densities, which cause a rapid discharge of hydrogen, are apt to yield loosely adherent deposits of spongy metal. A current density which gives a bright red, coherent deposit of pure copper when no interfering substance is present, will often give a very dark, loosely adherent deposit when arsenic is present, even in small amount. Such impurities must be removed before the electrolysis is begun. 
II. If the sample to be analyzed by this method is a copper ore, and is not known to be free from arsenic and other interfering substances, it should be subjected to special treatment, in order to obtain a solution suitable for electrolysis. In most cases, a satisfactory solution may be prepared according to the procedure detailed under the volumetric estimation of copper (which see); the ore is evaporated with aqua regia, the residue extracted with dilute hydrochloric acid and water, and the copper, arsenic, etc., precipitated with sodium thiosulphate; the arsenic is then driven off by ignition, the residue evaporated to dryness with nitric acid, and finally taken up in 4 cc. of nitric acid (sp. gr., r.42) and 50 cc. of water. This solution is diluted to $100 \mathrm{cc}$. and electrolyzed.

12. In electro-analysis, owing to its resistance to attack by the electrolytic solutions, platinum has been the material most often used for electrodes. The present-day price of this metal, however, tends greatly to encourage the use of other materials. Other metals, as silver and copper, are in some cases suitable for use as cathodes in the deposition of metals (as is also the more expensive palladium-gold alloy which is in the market), but the anode must still be made of platinum or of something equally resistant. In the determination of copper, for example, a silver cathode is just as satisfactory as one of platinum; the deposit should be removed in the cold by means of dilute hydrochloric acid, with the addition of a little hydrogen peroxide or nitric acid, and, after washing with ammonia, the cathode is again ready for use.

Since an upper limit is set for the current density, the time necessary for the deposition of a metal decreases with the increasing area of the cathode; for this reason the cathode should offer a large surface for the deposit. A thin platinum dish has a comparatively large inner surface, and is especially satisfactory for certain determinations. A platinum disk or a flat spiral of platinum wire may be used as the other electrode. The electrodes commonly used, however, are more economical. They are hollow cylinders of thin foil or of fine mesh gauze, and elongated spirals of heavy platinum wire; the cylinders, used to receive the deposit, if of platinum, weigh IO-I $2 \mathrm{~g}$., and the wire spirals about $8 \mathrm{~g}$. The gauze cylinders are the most efficient; they present a larger surface, all parts of which equally receive the deposit, and they allow a free circulation of the electrolyte and consequently the use of higher current densities.

These cylindrical cathodes and spiral anodes are the ones assumed in the foregoing procedures. The use of silver cathodes is advocated in the determination of copper, but they should not be heated with the hydrochloric acid-hydrogen peroxide cleaning mixture, owing to the solvent action in that case. 
13. In case the electrolysis is to be performed with stationary electrodes, it is best to use a tall beaker of small diameter, which can be heated. If heating is not desired, however, or if a rotating electrode is used, the most suitable vessel is a r50-cc. glass cylinder, with a rounded bottom ending in an outlet tube provided near the top with a stopcock; the electrodes should reach nearly to the bottom of this cylinder, to insure efficient mixing. After the deposition is complete, without interrupting the current, the electrolyte can easily be drawn off with the simultaneous introduction of distilled water above. 


\section{PART III \\ VOLUMETRIC ANALYSIS}

\section{GENERAL DISCUSSION}

Fundamental Principles. In volumetric analysis, the quantity of an element or compound present in a weighed sample is determined by calculation from the volume of a dissolved reagent of accurately known concentration which is required to complete a definite reaction. The concentration or value of the solution for producing a given reaction is determined by an operation known as standardization; the solution is caused to react, under favorable conditions, with a known weight of a definite substance and, from the volume of the solution required, its reaction value may readily be calculated. This solution is then called a standard solution.

The value of standard solutions may be expressed in terms of the weight of reagent actually present in each cubic centimeter, or, better, in terms of the weight of a given substance with which one cubic centimeter of the solution will react. But since the weight of reagent present in a unit-volume is always chemically equivalent to the weight of substance with which the unit-volume reacts, it is in general more convenient to express the value of the standard solution in terms of chemical equivalents per unitvolume; i.e. in terms of a normal solution. Such solutions, for example, may be made up to half-normal tenth-normal, or fiftieth-normal concentration. ${ }^{1}$

1 The value of the standard solution to be used in a specific case will depend largely upon the weight of the sample involved in the titration. In the estimation of acids and bases in usual amounts, it is customary to use half-normal solutions; 
A normal solution contains in one liter one gram-equivalent of the active reagent; i.e. that quantity which is chemically

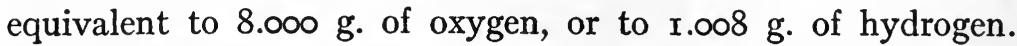
Thus, a normal acid solution contains $\mathrm{I} .008 \mathrm{~g}$. of available hydrogen-ion per liter (e.g. one mol of $\mathrm{HCl}$, or one half $\mathrm{mol}$ of $\mathrm{H}_{2} \mathrm{SO}_{4}$ ); and a normal alkali solution contains in one liter sufficient available hydroxide-ion to combine with $1.008 \mathrm{~g}$. of hydrogen-ion, or $\mathrm{I} 7.008 \mathrm{~g}$. (e.g. one mol of $\mathrm{NaOH}$, one half $\mathrm{mol}$ of $\mathrm{Ba}(\mathrm{OH})_{2}$, etc.). A normal oxidizing solution has an oxidizing value per liter of 8.000 g. of oxygen (e.g. one gram-atom of available iodine, one sixth mol of $\mathrm{K}_{2} \mathrm{Cr}_{2} \mathrm{O}_{7}$, etc.) ; and a normal reducing solution has a reducing value per liter of $\mathrm{I} .008 \mathrm{~g}$. of hydrogen (e.g. one mol of $\mathrm{FeSO}_{4}$, one half $\mathrm{mol}$ of $\mathrm{SnCl}_{2}$, etc.). It will be seen that (if they react quantitatively) equal volumes of normal acid and alkali will exactly neutralize one another, equal volumes of normal oxidizing and reducing reagents will exactly use up one another, etc.

Nevertheless, it must be realized that the equivalent or normal weight of a reagent may not always be the same. Thus the normal weight of oxalic acid is one half its molecular weight, whether used as an acid, as a reducing agent, or as a precipitating agent ; but the normal weight of nitrous acid would be its molecular weight if it were used as an acid, or to oxidize hydriodic acid, while if used to reduce potassium permanganate it would be only half as large. In the case of potassium permanganate, one molecule yields 5 equivalents of available oxygen in the titration of ferrous iron, but only 3 in the titration of manganese;

- the normal weight of this oxidizing agent may therefore be either one fifth or one third of the molecular weight, depending upon the reaction.

Although for special purposes it is sometimes worth while to prepare exactly normal or tenth-normal solutions, it is in general much easier, and just as satisfactory, to prepare and use stand-

but in the determination of small amounts, tenth-normal solutions give more accurate values, since their state of dilution minimizes the effect of errors of measurement. 
ard solutions which closely approximate these in value. In practice, the volumes used of such solutions can readily be expressed in terms of those of exactly normal (or, for that matter, tenth-normal) concentration; e.g. $25.75 \mathrm{cc}$. of a $0.0987 \mathrm{~N}$ solution are equivalent to $25.75 \times 0.0987=2.542 \mathrm{cc}$. of the normal, or to $2.542 \times 10=25.42 \mathrm{cc}$. of the tenth-normal solution.

The Fundamental Reactions of Volumetric Analysis. Volumetric analytical processes are usually based upon sharply defined chemical reactions which can be made to run rapidly to completion in aqueous solution. This, however, is not always effected by direct titration, i.e. by simply bringing the reacting substances together in equivalent quantities. In many cases a standard solution is first added in appreciable excess, and the excess then titrated with a second standard solution. In other cases the substance is treated with some compound with which it reacts to set free, or to carry down as an insoluble precipitate, a definite proportion of some other substance; and the latter is subsequently titrated with a standard solution.

According to the nature of the titration reactions themselves, volumetric analysis is subdivided as follows:

A. Neutralization Methods; utilized for the determination of acids and bases, and therefore called methods of acidimetry and alkalimetry.

B. Methods of Oxidation and Reduction; of which the most important are the permanganate, and iodometric processes e.g. the determination of ferrous iron or of manganese by means of potassium permanganate.

C. Precipitation Methods; as, for example, the determination of silver by means of sodium chloride solution.

The reactions employed in connection with these processes are in general of the reversible type. Of these, the reactions of neutralization run to completion in consequence of the low degree of ionization of one product, water; those of oxidation and reduction, in consequence of the relative potentials of the oxidizing and reducing substances under specific experimental condi- 
tions; and the reactions of precipitation, owing to the formation and precipitation of substances of an insoluble nature.

Determination of the End-Point. In order to utilize a reaction for purposes of titration, it is necessary to have a means of recognizing the point at which an equivalent quantity of the standard solution has been added. In approaching or passing this point certain properties of the solution are subject to rapid change; there may be a sudden change in the color, the electrical conductivity, or the oxidation potential of the solution, or a precipitate may cease to form. In numerous instances the presence of a substance purposely added, called an indicator, may give rise to a well defined color change in the solution, or it may cause a precipitate to form and thus render the solution cloudy at the end. The point to which the standard solution must be added in order to make such a change apparent is called the end-point of the titration. The process is the more satisfactory the smaller the difference between this point and the point at which an exact equivalent of the titration reagent has been added; in most of the established volumetric processes, this difference is exceedingly small.

In a few cases, in which there is an appreciable difference between the true and the observed end-point of a reaction, it is possible, by taking advantage of the principle of compensating errors, to obtain very accurate results; any errors which are involved in the actual analysis are offset by equal errors made in the standardization. In general, the difference between the true and the observed end-point will remain constant under the same specific conditions; if, therefore, the standardization and the analysis are both performed under identical conditions, the standard solution simply serves as a means of comparing two approximately equal quantities of the same substance, one of which is accurately known. From the data of standardization, for example, we calculate the value of the solution per cubic centimeter in terms of the substance; and then, by multiplying the number of cubic centimeters consumed in the titration 
by this value, we obtain the weight of the substance contained in the sample analyzed.

Bearing this in mind, it will be seen that, at least from a theoretical standpoint, the highest attainable accuracy cannot be expected in a given case unless the standard solution used has had its value determined in terms of the particular substance under investigation, and under conditions in every way identical with those prevalent throughout the analysis.

General Theory of Indicators. When a substance is titrated in the presence of an indicator, the end-point is recognized as the visible effect of a chemical change in which the indicator itself takes an active part. Either the indicator may combine with the reagent in the standard solution (when the latter has been added in slight excess), or it may enter into combination with a portion of the substance undergoing titration, to be again liberated in colorless form upon the addition of the standard solution. These two cases are exemplified in the behavior of starch as an indicator, in the processes of iodometry; in those titrations which are made with iodine, a blue color appears and persists upon the addition of the slightest excess of iodine, while in titrations of iodine with sodium thiosulphate solution, for example, the blue color of the iodo-starch is discharged upon the addition of the thiosulphate.

In those cases in which the reagent acts upon the indicator, the reactions involved in the titration may be represented by the following equations, in which $S$ is the substance titrated, $R$ the reagent in the standard solution, and $I$ the indicator :
(a) $S+R \lessgtr R S$,
(b) $I+R \leftrightarrows R I$,
(c) $S+R I \supsetneqq R S+I$.

The recognition of the end-point depends upon the formation of a visible quantity of $R I^{1}$; this substance should be incapable

${ }^{1} R I$ is not necessarily a compound of the indicator with $R$, but even so a definite concentration of the free reagent $R$ must finally be present in the solution in order 
of existence in the presence of $S$, but after the concentration of $S$ has been lowered to an infinitesimal value, the concentration of $R I$ should increase very rapidly with the further addition of $R$. The small amounts of $R I$ formed locally in consequence of imperfect mixing should of course react with $S$ and insure the completion of the main reaction, before the indicator becomes permanently affected by $R$. The closeness of agreement between the observed and the true end-point under the conditions prevalent in any specific case will depend therefore upon the relative magnitudes of the equilibrium constants of these three reactions.

In those cases, however, in which the indicator combines with the substance undergoing analysis, the mechanism of the titration may be represented as follows :

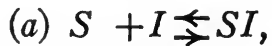
(b) $S+R \leftrightarrows R S$,
(c) $S I+R \leftrightarrows R S+I$.

Here the end-point is recognized by the visible disappearance of $S I$ from the solution. In a few cases, in which $S I$ will not react with $R$, it is necessary to use a special procedure; as the end-point is approached, the solution is frequently tested, a drop at a time on a test-plate, with a drop of the indicator solution (see the determination of ferrous iron with potassium dichromate solution).

A factor which is often of the greatest importance is the concentration of the indicator in the solution. In the reactions of neutralization, in which the end-point is most frequently recognized by a sudden change from one color to another (or to colorless) in the solution, only a very slight amount of indicator should be used $;^{1}$ otherwise the entire quantity would not be trans-

to produce the visible change which is characteristic of the indicator. In such cases reaction $(b)$ may be written $I_{1} \underset{\leftarrow}{\gtrless} I_{2}$, and the appearance of the end-point is dependent upon the concentration of $I_{2}$, which in turn depends upon that of $R$ in the solution.

${ }^{1}$ In the case of methyl orange, for example, the indicator is prepared by dissolving $0.02-0.03 \mathrm{~g}$. of the solid compound in roo cc. of water, and in any one 
formed by a slight enough excess of the reagent, and there would be a more gradual change of color. On the other hand, if $R I$ is a compound subject to dissociation, it is often necessary, in order by mass action to insure its prompt formation, to add the indicator in considerable amount. (Cf. the use of starch in iodometry, and of ferric-ion in the titration of silver with thiocyanate solution.)

Volumetric vs. Gravimetric Methods. Volumetric methods are usually more rapid and convenient of execution than gravimetric. The titration itself takes only a short time; but the preliminary removal of interfering substances and the transformation of the substance into a form suitable for titration may require several hours. Some volumetric processes are more accurate, and others less accurate, than the corresponding gravimetric processes. They often avoid errors due to solubility, to the contamination of precipitates, and to mechanical losses, which are inherent in the gravimetric processes; but they involve certain other errors in the volumetric measurement of liquids and in the determination of end-points.

General Remarks. The student should carefully study the discussion in Part I concerning the sources of error in the volumetric measurement of liquids. In the way of summary, the following points may be emphasized.

All measuring vessels should at the same temperature be based upon the same standard of volume, and the conditions observed in their use should be identical with those which prevailed in their calibration. Also, in working with standard solutions, the conditions prevalent during standardization should always be observed in analysis; this applies especially to the temperature, to the final volume after titration, and to the amount of indicator used. The value of the solution is apt to vary appreciably with the experimental conditions.

titration only about three drops of this solution should be used. Counting 20 drops to $\mathrm{I}$ cc., and using 3 drops in a titration, the amount of methyl orange actually present is less than $0.05 \mathrm{mg}$. 
It is of course necessary to control the value of the standard solutions. They should be preserved in closely stoppered bottles at a fairly constant temperature, in some instances in the dark. Before use, the bottle should be shaken, to cause the solution to again take up any liquid that may have evaporated and condensed upon the glass; and, after use, before replacing the stoppers, the necks and stoppers of the bottles should be wiped dry with a lintless cloth. In addition to all this, solutions subject to loss by volatilization or to chemical change, should have their value controlled at suitable intervals by restandardization.

Measuring vessels should be clean and free from grease films, and before filling, they should be washed out with three separate small portions of the solution to be measured, and the washings discarded (through the tip, in the case of burettes and pipettes).

In general, economy of time should be practiced by calculating roughly the volume of the standard solution which will be needed in a titration, and then running in almost this quantity from the burette; this can be followed by a careful determination of the end-point. In case such a calculation cannot be made, it is often worth while to run a very rapid preliminary titration, in order to approximately fix this volume (see, for example, the determination of manganese by titration with potassium permanganate). 


\section{A. NEUTRALIZATION METHODS}

\section{ACIDIMETRY AND ALKALIMETRY}

Fundamental Principles. An aqueous solution which is neutral contains $\mathrm{H}^{+}$and $\mathrm{OH}^{-}$ions in equal concentration, and, so long as the solution is very dilute, the number of mols of water ionized in one liter is $0.000000 \mathrm{I}$, or $\mathrm{IO}^{-7}$, at $25^{\circ}$. Since in the ionization equilibrium, $\mathrm{H}_{2} \mathrm{O} \leftrightarrows \mathrm{H}^{+}+\mathrm{OH}^{-}$, $\left(\mathrm{H}^{+}\right)\left(\mathrm{OH}^{-}\right)=k$, it follows that in dilute solutions $\left(\mathrm{H}^{+}\right)\left(\mathrm{OH}^{-}\right)=$ IO ${ }^{-14}$.

If an acid is added to water, or to a dilute neutral solution, in sufficient amount to increase the $\mathrm{H}^{+}$-ion concentration from $\mathrm{IO}^{-7}$ to $\mathrm{ro}^{-6}$, then the $\mathrm{OH}^{-}$-ion concentration will fall to $\mathrm{IO}^{-8}$. Only $0.0000009 \mathrm{~mol}$ of $\mathrm{H}^{+}$ion would be required to produce such a change in a liter of neutral solution, and this amount is contained in about 0.01 cc. of tenth-normal hydrochloric acid, while the addition of only $0.1 \mathrm{cc}$. to one liter would increase the $\mathrm{H}^{+}$-ion concentration from $\mathrm{IO}^{-7}$ to $\mathrm{IO}^{-5}$.

The indicators commonly used to determine the end-point in processes of acidmetry and alkalimetry are rather complicated organic compounds, each capable of existence in two forms of different color, - these forms being mutually convertible, one into the other, at specific $\mathrm{H}^{+}$- and $\mathrm{OH}^{-}$-ion concentrations.

The color changes which are characteristic of these indicators do not as a rule occur in exactly neutral solutions. The accompanying table gives for some of the more common indicators, the $\mathrm{H}^{+}$-ion concentrations at which the color changes occur.

It might seem, at first thought, that only an indicator which changes exactly at the neutral point would be suitable, but this is by no means true. In titrating a strong acid against a strong base a few hundredths of a cubic centimeter of tenth-normal acid or alkali in excess will carry the concentration of hydrogen or hydroxide ion so far to one side of the neutral point that any 
of the indicators for which the characteristic point lies between $10^{-5}$ and $10^{-9}$ will give a sharp and accurate end-point.

In titrating a weak acid with a strong base, e.g. acetic acid with sodium hydroxide, the acetate ions from the highly ionized sodium acetate drive the reaction, $\mathrm{HC}_{2} \mathrm{H}_{3} \mathrm{O}_{2} \underset{\lessgtr}{\lessgtr} \mathrm{H}^{+}+\mathrm{C}_{2} \mathrm{H}_{3} \mathrm{O}_{2}^{-}$, far to the left, long before an equivalent quantity of the base has been added; the concentration of hydrogen ion becomes exceedingly low $\left(<\mathrm{IO}^{-5}\right.$ but $\left.>\mathrm{IO}^{-7}\right)$ before the major portion of the acetic acid has had its hydrogen replaced. In such a case the change in color for Methyl orange $\left(\mathrm{IO}^{-4}-\mathrm{IO}^{-5}\right)$ will appear gradually, during the addition of a cubic centimeter or more of the alkali, long before an equivalent quantity has been run in, and no sharp end-point will be indicated. If phenolphthalein $\left(10^{-8}-\mathrm{IO}^{-7}\right)$ is used, however, the change in color will not occur until the true neutral point, $\left(\mathrm{H}^{+}\right)=\mathrm{IO}^{-7}$, has been passed; a very slight excess of alkali will then reduce the concentration of the hydrogen ion far below $10^{-8}$, a sharp end-point will be indicated, and the total alkali added will correspond very exactly to that required for the actual neutralization of the acid.

When a weak base is titrated with a strong acid, e.g. ammonium hydroxide with hydrochloric acid, the conditions are reversed and such indicators as methyl orange, methyl red, or cochineal, which change color in a faintly acid solution $\left(\right.$ i.e. $\left.\mathrm{H}^{+}>\mathrm{IO}^{-7}\right)$ are most suitable.

The following rules should be observed in the use of neutralization methods for the determination of acids and bases:

r. In the titration of a strong acid with a strong base, or vice vers $a$, use any indicator in the list, from methyl orange to phenolphthalein.

2. In the titration of any acid other than a strong mineral acid with a strong base, use phenolphthalein, trinitrobenzene, or a similar indicator.

3. In the titration of a weak base with a strong acid, use methyl orange, Congo red, or a similar indicator.

4. Do not attempt to titrate a weak base against a weak acid. 


\begin{tabular}{|c|c|c|c|c|c|c|c|c|c|c|c|c|c|c|}
\hline$\overline{\mathrm{i}}$ & & & & & & & & & & & & + & + & ఫ̈ \\
\hline 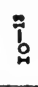 & & & & & & & & & & + & + & + & 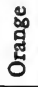 & + \\
\hline 莕 & & & & & & & & & + & + & + & 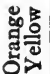 & + & + \\
\hline $\overrightarrow{\tilde{D}}$ & & & & & & & & + & + & + & + & + & + & + \\
\hline î & & & + & & & & + & + & + & + & $\stackrel{g}{\underline{m}}$ & + & + & 产 \\
\hline io & + & + & + & & + & & + & + & + & $\underset{\mathscr{Z}}{\mathscr{Z}}$ & + & + & 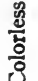 & \\
\hline إْ & + & + & + & + & + & + & 邑 & छ̈ & 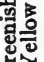 & + & + & + & & \\
\hline î & + & + & + & + & 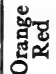 & + & 苂 & 芯 & + & + & + & 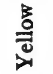 & & \\
\hline i & + & + & $\underset{\&}{\mathbb{Z}}$ & 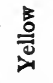 & 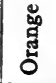 & 恚 & 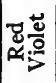 & + & + & + & $\begin{array}{l}\frac{0}{0} \\
\frac{0}{0} \\
0\end{array}$ & & & \\
\hline o & + & 亳 & + & 总 & 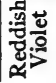 & 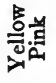 & "ृ & + & + & $\begin{array}{l}\text { 总 } \\
\frac{0}{0}\end{array}$ & & & & \\
\hline $\begin{array}{l}T \\
\\
\end{array}$ & 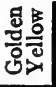 & 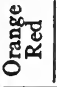 & + & + & 苋 & 喜 & + & + & 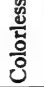 & & & & & \\
\hline i & 存焉 & + & + & $\ddot{\varpi}$ & 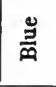 & + & + & 善 & & & & & & \\
\hline ז & + & + & + & + & + & + & + & & & & & & & \\
\hline 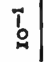 & + & + & + & & & & & & & & & & & \\
\hline & 焉 & 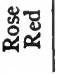 & 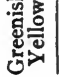 & & & & & & & & & & & \\
\hline 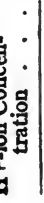 & 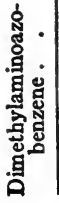 & 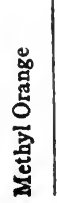 & 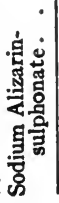 & 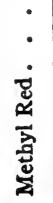 & 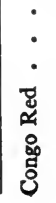 & 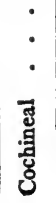 & $\begin{array}{l}\dot{\cdot} \\
\dot{\cdot} \\
\dot{g} \\
\dot{3}\end{array}$ & 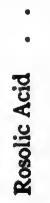 & 苞 & 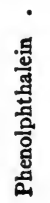 & 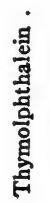 & 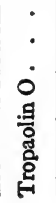 & & 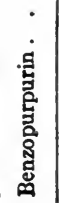 \\
\hline
\end{tabular}

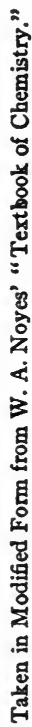


The sensitivity of a given indicator may vary widely with the temperature or dilution, and for that reason the end-point should be determined in about equal volumes of solution, both in standardization and in analysis $;^{1}$ and when, as is often necessary, a solution is to be titrated at the boiling temperature, the standardization also should be carried out at that temperature. And, since a slight amount of acid or of alkali is necessary to transform the indicator, the constancy of this amount should be insured by always using a fixed quantity of the indicator solution, say 3 drops.

Methyl orange solution is most readily prepared by dissolving $0.02-0.05 \mathrm{~g}$. of the solid compound (also known as Orange III) in a very little alcohol and diluting with water to roo cc. It is often used in the titration of strong acids and bases, and especially in the determination of weak bases, as ammonium hydroxide. It is also serviceable in titrating with a strong acid the salts of very feeble acids, as carbonates, sulphides, borates, etc.; in such cases the acids set free are too slightly ionized to affect the indicator, and the change to pink does not occur until the standard acid has been added in veryslight excess. In reality, the strong acid simply rémoves the ${ }^{-} \mathrm{H}^{-}$-ions which accumulate in the solution in consequence of the hydrolysis of the salt, and thus drives the hydrolysis to completion: e.g.

\section{$\mathrm{Na}_{2} \mathrm{SiO}_{3}+2 \mathrm{HOH} \underset{2}{\lessgtr} \mathrm{Na}^{+}+\mathrm{H}_{2} \mathrm{SiO}_{3}+2 \mathrm{OH}^{-}$.}

The use of methyl orange should be confined to cold solutions, which as a rule are to be titrated by standard acid or alkali of about normal or half-normal concentration.

Phenolphthalein solution is prepared by dissolving about I g. of the solid in $55-60 \mathrm{cc}$. of $95 \%$ alcohol, and diluting the solution to $100 \mathrm{cc}$. This indicator is especially adapted to the titration of slightly ionized acids, as acetic and tartaric acids. (It should not be used in connection with weak bases, such as

${ }^{1}$ In this connection, see G. P. Baxter, Jour. Amer. Chem. Soc., Vol. 36, p. 656 (I9I4). 
ammonia.) It is decolorized even by carbonic acid, which therefore must be removed by heating when other substances are being determined; unlike methyl orange, it is sensitive in boiling hot solutions.

Standard Acid Solutions. These are generally prepared from hydrochloric or sulphuric acid. Hydrochloric acid is preferable to sulphuric in the titration of barium hydroxide; but, on the other hand, solutions of sulphuric acid can be used in titrations at the boiling temperature without danger of loss. Both acids may be used with any indicator in the table from methyl orange to phenolphthalein.

Standard Alkali Solutions. These are mostly prepared from sodium hydroxide, but sodium carbonate and barium hydroxide are sometimes used. Sodium hydroxide, if free from carbonate, may be used with any indicator; but its solutions absorb carbon dioxide from the air. Pure sodium carbonate, accurately weighed out, may be dissolved in water and diluted to give a standard solution, but its usefulness may be impaired by the liberation of carbonic acid. Barium hydroxide solutions are free from carbonate, since any carbon dioxide absorbed is precipitated as insoluble barium carbonate; this, however, lowers the concentration of the base. Barium hydroxide may be used with any indicator, from methyl orange to phenolphthalein, but it is not soluble much above one fourth normal; if a more concentrated solution of carbonate-free alkali is desired, however, a sodium hydroxide solution may be freed from carbonate by means of a slight excess of barium chloride, and then standardized. Carbonate-free solutions should be protected from carbon dioxide by means of a soda-lime absorption tube.

THE PREPARATION AND STANDARDIZATION OF APPROXIMATELY HALF-NORMAL SOLUTIONS OF HYDROCHLORIC ACID AND SODIUM HYDROXIDE

Procedure. Pour into a roo cc. measuring cylinder a volume of hydrochloric acid (e.g. 82.5 cc. of the acid of sp. gr. r.I9) 
sufficient to contain $36.5 \mathrm{~g}$. of hydrogen chloride, transfer it quantitatively to a one-liter measuring flask, and dilute to the mark with distilled water. Pour this solution without loss into a clean, well-drained 2.5 liter bottle, refill the measuring flask with water, and pour this also into the bottle. Finally mix the solution thoroughly by vigorous shaking in the stoppered bottle.

In a large beaker, dissolve in water about $42 \mathrm{~g}$. of stick sodium hydroxide, weighed on a rough balance, dilute this solution also to 2 liters, and shake the mixture in the stoppered bottle.

Allow these solutions to stand, best overnight, until they have attained the temperature of the laboratory.

Fill two clean burettes with the respective solutions, first thoroughly rinsing each burette with one ro cc., and two $5 \mathrm{cc}$. portions of the corresponding solution, each time draining off the wash liquid through the tip. See that the tips are free from air, and then record the exact reading of each burette. Run out about $20 \mathrm{cc}$. of the acid into a $500 \mathrm{cc}$. Erlenmeyer flask, add $60 \mathrm{cc}$. of water and 3 drops of methyl orange solution, and then from the other burette run in alkali, with shaking, until the color of the solution changes from pink to yellow. Wash down the inside of the flask with a little water, replace it under the first burette, and add acid until the solution turns pink. In this way, accurately adjust the mixture until a drop of either acid or alkali will cause a definite change of color. Select the point at which the faintest tinge of pink appears as the end-point of the titration; later on, always titrate to this point. Allow about 30 seconds for afterflow, and then record the readings of the burettes. Having corrected the readings in accordance with the burette calibrations, and with the temperature if necessary (see Part I), calculate the ratio of the solutions as shown in the following example :

$$
\frac{\text { cc. acid }}{\text { cc. alkali }}=\frac{21.53}{21.07}=1.022 \text { cc. of acid per } 1.000 \text { cc. of alkali. }
$$

Fill the burettes again, and repeat the operation. The duplicate ratios should check within two parts in one thousand. 
With this ratio accurately established, standardize the hydrochloric acid solution as follows:

(a) By Titration against Pure Sodium Carbonate. If a suitable oven is available, dry the salt on a watch glass for an hour at $130-150^{\circ}$; otherwise heat about ro g. of pure sodium carbonate in a small porcelain dish, on a wire gauze over a small Bunsen flame, for one half hour, and then allow the salt to cool in a desiccator. Transfer the cold salt to a dry, well-stoppered weighing tube, and weigh out into $500 \mathrm{cc}$. Erlenmeyer flasks two portions of $0.5-0.6 \mathrm{~g}$. each, ${ }^{1}$ tecording the exact weights. Add to each sample $80 \mathrm{cc}$. of water and 3 drops of methyl orange solution, and shake until dissolved. With both burettes full and the readings recorded, run in the acid with shaking, until the solution just turns pink. Wash down the inside of the flask, and, if the solution turns yellow, add acid a drop at a time, until the faintest tinge of pink returns. (If too much acid is added, the excess can be determined by means of the alkali in the other burette, since the ratio of the solutions is known.) After a few moments, read the burettes and record the readings. From the data obtained in each case, calculate the normality factor of the acid; and also that of the alkali, using the mean of the duplicate values obtained in determining the ratio of the two solutions. The two results should agree within two parts in one thousand.

(b) Gravimetrically with Silver Nitrate. Measure out accurately from a pipette ro.o0 cc. of the acid into each of two $300-c c$. beakers, and dilute in each case with $150 \mathrm{cc}$. of water. Precipitate the chlorine from these solutions with silver nitrate according to the procedure given in Part II, and filter the silver chloride off through Gooch crucibles, prepared and weighed as there indicated. Wash the precipitates with hot water until free from soluble silver salts, dry at $\mathrm{I} 20-\mathrm{I} 30^{\circ}$ to constant weight, and from the weight of silver chloride found in each case calcu-

1 The weights of samples in this book are based upon the use of $30-\mathrm{cc}$. burettes. If 50-cc. burettes are used, it is better to take samples $\frac{5}{3}$ as large. 
late the normality factor of the acid. The duplicate values should agree very closely with one another, and also with those previously found by titration against sodium carbonate.

Notes. - I. Although silver chloride is insoluble, the normality factor of the hydrochloric acid may nevertheless be calculated directly from the weight of the precipitate obtained. For example, if $10.00^{\prime} \mathrm{cc}$. of the acid were found to yield $0.7317 \mathrm{~g}$. of $\mathrm{AgCl}$, then (since an equal volume of normal $\mathrm{HCl}$ would yield $\mathrm{I} .4334 \mathrm{~g}$. of $\mathrm{AgCl}$ ) the normality factor of the acid is $0.7317 / \mathrm{r} .4334$, or 0.5105 .

In the same way, the normality factor of the acid may be calculated in the case of Method (a). For example, if 0.5682 g. of $\mathrm{Na}_{2} \mathrm{CO}_{3}$ require $21.00 \mathrm{cc}$. of acid, the normality factor is equal to $0.5682 / \mathrm{I} . \mathrm{II} 30=0.5 \mathrm{ro5}$ (I.Ir3O g. is the amount of $\mathrm{Na}_{2} \mathrm{CO}_{3}$ contained in $21.00 \mathrm{cc}$. of the normal solution).

If it has previously been found, for example, that $\mathbf{1} .000 \mathrm{cc}$. of alkali solution is equivalent to $1.022 \mathrm{cc}$. of the acid, then it follows that the normality factor of the alkali is $0.5105 \times 1.022=0.5217$.

2. If it is desired to prepare solutions of exactly one half normal concentration, slightly stronger solutions are first prepared, and, after standardization, they are diluted with the calculated volume of water. For example, the $0.5105 N$ acid should be diluted according to the proportion, $0.5105: 0.5000=x: 1000$, and the $0.5217 N$ alkali according to the proportion, $0.5217: 0.5000=y: 1000$; i.e. one liter of each solution should have added to it $21.0 \mathrm{cc}$. and $43.2 \mathrm{cc}$. of water, respectively. The water is added from a burette. After dilution the solutions should be thoroughly shaken, and then restandardized.

3. In fixing the end-point of the titration, it is somewhat easier to recognize the change from yellow to pink than that from pink to yellow. Whichever change is selected as the end-point, however, should be consistently made use of in all subsequent titrations.

4. There has been much discussion concerning the compound which is best suited for use in the standardization of acids. Although calcium carbonate has many advocates (see Part V, Problem 54), it is more customary to use sodium carbonate. This salt has the advantage of being soluble in water, and it can now be purchased sufficiently pure for the purpose. The pure salt can easily be prepared by heating the recrystallized bicarbonate at $280-300^{\circ}$, when it decomposes according to the equation,

$$
2 \mathrm{NaHCO}_{3}=\mathrm{Na}_{2} \mathrm{CO}_{3}+\mathrm{H}_{2} \mathrm{O}+\mathrm{CO}_{2} \text {. }
$$

5. Of course it is possible to standardize the alkali solution directly, rather than the acid. With phenolphthalein as the indicator, this may be 
done against any of the following pure acids: oxalic acid, $\mathrm{H}_{2} \mathrm{C}_{2} \mathrm{O}_{4}{ }_{2} \mathrm{H}_{2} \mathrm{O}$; acid potassium oxalate, $\mathrm{HKC}_{2} \mathrm{O}_{4} \cdot \mathrm{H}_{2} \mathrm{O}$; potassium tetroxalate, $\mathrm{H}_{3} \mathrm{~K}\left(\mathrm{C}_{2} \mathrm{O}_{4}\right)_{2}$ . $2 \mathrm{H}_{2} \mathrm{O}$; succinic acid, $\mathrm{H}_{2} \mathrm{C}_{4} \mathrm{H}_{4} \mathrm{O}_{4}$; acid potassium tartrate, $\mathrm{HKC}_{4} \mathrm{H}_{4} \mathrm{O}_{6}$; acid potassium phthalate, $\mathrm{HKC}_{8} \mathrm{H}_{4} \mathrm{O}_{4}$. The last two are probably the most suitable, since they are monobasic acids of high molecular weight, do not contain water of crystallization, and are easily obtainable in a pure condition.

6. While it is permissible to standardize hydrochloric acid solutions (providing they are free from interfering impurities) gravimetrically with silver nitrate, solutions of sulphuric acid should not be standardized by precipitation as barium sulphate; in this case the purity of the precipitate is too open to question.

7. Before beginning work in volumetric analysis, the student should read Section VI of Part I, as well as the general discussion at the beginning of Part III. 


\section{THE DETERMINATION OF THE ALKALINE VALUE OF SODA}

The sample may be one of commercial soda, or it may be an artificial mixture of sodium carbonate and sodium chloride.

Procedure. Weigh out roughly on a clean watch glass about $5 \mathrm{~g}$. of the soda, dry it for I hour at $110^{\circ}$, and allow to cool in a desiccator. Now accurately weigh the sample on the watch glass, transfer it quantitatively to a beaker, and dry and weigh the watch-glass in order to arrive at the exact weight of the sample.

Warm the sample with roo cc. of water and, after cooling, filter from any insoluble matter, thoroughly washing the filter with cold water and receiving the filtrate and washings in a clean $250 \mathrm{cc}$. measuring flask. At the room temperature, dilute to the graduation mark with water (see p. 45 , Parallax), adding the latter gradually and with shaking; and then, using a clean dry beaker, thoroughly mix the solution by pouring it back and forth from one vessel into the other.

Measure out two or three $25 \mathrm{cc}$. portions of the liquid, by means of a pipette previously rinsed with the solution, into 500 cc. Erlenmeyer flasks, touching the tip of the pipette for final drainage each time on the inner wet surface of the vessel (unless it was calibrated otherwise). Add $50 \mathrm{cc}$. of water and 3 drops of methyl orange solution, and titrate with the standard acid, as in the standardization of the latter.

From the (corrected) volumes of acid and alkali used, remembering that only one tenth of the sample was used in each titration, calculate the quantity of alkali present in terms of pure sodium carbonate, and report the percentage of the latter found.

Notes. - I. Let us assume, for example, that $5.890 \mathrm{~g}$. of soda were used in the preparation of $250.0 \mathrm{cc}$. of solution, and that $25.75 \mathrm{cc}$. of $0.5105 \mathrm{~N}$ acid and $4 . \mathrm{I}_{3} \mathrm{cc}$. of $0.5217 \mathrm{~N}$ alkali were used in the titration of $25.00 \mathrm{cc}$. of this solution. Then it follows that $5.890 \mathrm{~g}$. of the soda are equivalent to $10 \times(25.75 \times 0.5105-4.13 \times 0.5217)=109.90 \mathrm{cc}$. of $N$ acid; and, since this volume of normal acid would neutralize an equal volume of normal alkali, therefore the $5.890 \mathrm{~g}$. of soda contained $0.053 \times$ rog.90 $=5.8246 \mathrm{~g}$., or $98.9 \%$ of $\mathrm{Na}_{2} \mathrm{CO}_{3}$. 
2. Soda is apt to contain varying amounts of water, and, for the sake of uniformity, the sample to be analyzed is ordinarily dried for a specified time at $110^{\circ}$; though this treatment is insufficient to completely remove the moisture.

3. Crude Solvay soda usually contains as impurities, besides a small amount of sandy grit, also small quantities of sodium chloride and sulphate, and either the bicarbonate or hydroxide. Either of the latter two has an alkaline value of its own, but nevertheless the alkaline value is ordinarily expressed in terms of sodium carbonate alone.

4. The chemical action in the titration of sodium carbonate with hydrochloric acid consists essentially of two stages; first the neutralization of hydroxide-ion, which accumulates in the solution in consequence of hydrolysis, and second the conversion of the bicarbonate-ion of the first reaction into non-ionized carbonic acid. The carbonic acid itself is too feeble to have any effect upon methyl orange; it decomposes mostly into water and carbon dioxide.

(a)

$$
\begin{aligned}
& \begin{array}{r}
\mathrm{Na}_{2} \mathrm{CO}_{3}+\mathrm{HOH} \underset{\mathrm{HCl}}{\stackrel{5}{\leftrightarrows}} \mathrm{Na}^{+}+\mathrm{HCO}_{3}^{-}+\mathrm{OH}^{-} \\
\mathrm{Cl}^{-}
\end{array}
\end{aligned}
$$

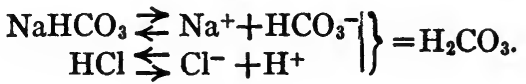

5. For other methods of analyzing soda, the student is referred to Part V, Problems 60, 6r, and 96. 
THE DETERMINATION OF AVAILABLE HYDROGEN-ION IN AN ACID

The sample may be oxalic acid, acid potassium oxalate, potassium tetroxalate, succinic acid, acid potassium phthalate, potassium bitartrate, or something similar.

Procedure. Weigh out accurately into $500 \mathrm{cc}$. Erlenmeyer flasks $0.6-0.7 \mathrm{~g}$. portions of the unknown acid, and treat each as follows. Warm the sample with $80 \mathrm{cc}$. of water, add 3 drops of phenolphthalein solution, and with shaking, run in the standard alkali until a pink color persists. Cautiously add the standard acid, from a second burette, until the color just vanishes, and then an excess of $0.3-0.4 \mathrm{cc}$. Heat the solution for 3 minutes at the boiling temperature, and, if the color reappears add acid as before to the hot solution, again $0.3-0.4 \mathrm{cc}$. in excess, and boil again for 3 minutes. Continue this treatment until the pink color is not restored by boiling for 3 or 4 minutes. Finally add alkali until the faintest tinge of pink returns, then 2 drops of acid, and boil, and if no color returns, finish the titration with alkali at the boiling temperature.

From the data obtained, calculate the normality factor of a solution containing $1.000 \mathrm{~g}$. of the unknown acid in $100 \mathrm{cc}$. The results should check within two parts in one thousand.

Notes. - r. Although, theoretically speaking, the same indicator (and temperature) should be used in standardization and analysis, the error involved in the non-observance of this principle is in this instance practically negligible.

2. Since phenolphthalein is sensitive to carbonic acid, and since the ordinary standard alkali always contains carbonate, it is necessary to expel this acid by boiling the solution. In a cold dilute solution of sodium bicarbonate which is saturated with carbon dioxide, phenolphthalein is colorless; i.e. in cold dilute solutions of sodium carbonate this indicator becomes colorless as soon as the carbonate has been transformed into bicarbonate by the addition of acid (see Note 4 of the preceding exercise). When this solution is heated, the bicarbonate is partially hydrolyzed,

$$
\mathrm{Na}^{+} \mathrm{HCO}_{3}-+\mathrm{H}^{+} \mathrm{OH}^{-} \rightleftarrows \mathrm{Na}^{+}+\mathrm{OH}^{-}+\mathrm{H}_{2} \mathrm{CO}_{3},
$$


and the solution loses carbon dioxide and becomes alkaline. This behavior will continue during the titration, as long as there is any bicarbonate left in the solution.

3. If present at any appreciable concentration, hydrogen chloride is more or less volatilized from boiling aqueous solutions; for this reason the acid should be added each time in very slight excess.

4. When a large number of determinations are to be made with phenolphthalein as the indicator, it is well worth while to prepare and standardize a carbonate-free alkali solution. The acid solutions in such cases should be boiled (unless there is danger of loss), to free them from carbonic acid, or they may be made up with freshly boiled water, and titrated hot with carbonate-free alkali. For the greatest accuracy, a specially constructed, steam jacketed titration vessel may be employed, in which case carbonate-free alkali is not really needed. 
THE DETERMINATION OF PROTEIN NITROGEN BY THE KJELDAHL METHOD

Principle. When an organic substance is heated with concentrated sulphuric acid, especially in the presence of an oxygen carrier, the organic substance is completely decomposed, and any protein (or other similarly combined) nitrogen is converted into ammonia. This at once combines with acid to form ammonium acid sulphate, $\mathrm{NH}_{4} \mathrm{HSO}_{4}$, which remains in solution in the sulphuric acid. Upon diluting the mixture with water and adding sodium hydroxide in excess, the ammonia is liberated, and can be distilled over and collected in a known volume of standard acid, which it partially neutralizes. By titrating the excess of acid with a standard alkali, the volume of the standard acid neutralized by the ammonia can be found, and from the data obtained the percentage of nitrogen in the sample may be calculated.

Procedure. Accurately weigh out from a weighing tube, upon separate sheets of quantitative filter paper, two samples of about I g. each of the substance to be analyzed. Wrap each sample carefully in the paper, and introduce the bundle into a clean 500 cc. Kjeldahl flask. To each flask add about 0.5 g. of powdered copper sulphate, and $25 \mathrm{cc}$. of concentrated sulphuric acid. See thiat the samples are thoroughly wet by the acid, and then place the flasks on the digestion rack in the Nitrogen Laboratory, with the necks resting in the circular openings of the lead ventilating pipe; place the flasks in unoccupied positions as near as possible to one of the exhaust flues. Heat gently until frothing ceases, add $10 \mathrm{~g}$. (weighed roughly) of potassium sulphate, or an equivalent weight of sodium sulphate, and heat to gentle ebullition for two or three hours until the liquid is of a clear green color, without any trace of brown (do not allow the flame to reach above the surface of the liquid). Continue the heating for half an hour longer, and allow to cool. 
While the flasks are cooling, accurately measure from a burette two 30.00-cc. portions of $0.5 \mathrm{~N}$. hydrochloric acid, into 400-cc. Erlenmeyer flasks, and add to each about $25 \mathrm{cc}$. of distilled water. Place these flasks under the distilling apparatus, so that the delivery tubes just dip into the acid solutions.

After cooling, carefully dilute the contents of the digestion flasks with I $_{50} \mathrm{cc}$. of distilled water, and cool again. Carefully pour down the inclined neck of each flask, so that it shall not mix with the acid solution, $75 \mathrm{cc}$. of sodium hydroxide solution ( 300 g. of $\mathrm{NaOH}$ per liter). Place the flasks on the distilling rack, add one or two pieces of granulated zinc, and quickly connect the flasks with the distilling heads, using well-fitting rubber stoppers. Finally, mix the contents of each flask by gently rotating it, and then begin to heat the mixture.

Distill off about two thirds of the contents of each flask, with great care that they do not boil over. The distillation will require about 45 minutes. Disconnect the distilling flasks and rinse out the delivery tubes into the receiving flasks with a little distilled water. Add 3 drops of methyl orange to each of the receiving flasks, and titrate the contents with $0.5 \mathrm{~N}$. sodium hydroxide.

From the data obtained, calculate the percentage of nitrogen in the sample.

Notes. - I. The sulphuric acid hydrolyzes the $\mathrm{NH}_{2}$-group, to give ammonia, and also acts as an oxidizing agent, converting the organic matter into carbon dioxide, water, or other volatile products. For example,

$$
\begin{gathered}
\mathrm{CO}\left(\mathrm{NH}_{2}\right)_{2}+\mathrm{H}_{2} \mathrm{O}+2 \mathrm{H}_{2} \mathrm{SO}_{4}=\mathrm{CO}_{2}+2 \mathrm{NH}_{4} \mathrm{HSO}_{4} ; \\
\mathrm{C}_{6} \mathrm{H}_{10} \mathrm{O}_{5}+n \mathrm{H}_{2} \mathrm{SO}_{4}=6 \mathrm{C}+5 \mathrm{H}_{2} \mathrm{O}+n \mathrm{H}_{2} \mathrm{SO}_{4} ; \\
\text { and } \mathrm{C}+2 \mathrm{H}_{2} \mathrm{SO}_{4}=\mathrm{CO}_{2}+2 \mathrm{H}_{2} \mathrm{O}+2 \mathrm{SO}_{2} .
\end{gathered}
$$

2. The $\mathrm{CuSO}_{4}$ gives up oxygen more readily to the organic matter than the $\mathrm{H}_{2} \mathrm{SO}_{4}$ does; but the $\mathrm{H}_{2} \mathrm{SO}_{4}$ then reoxidizes the copper so that at the end of the operation the copper is still present as copper sulphate. That is to say, the copper salt acts catalytically as an oxygen carrier.

3. Mercuric sulphate is often used instead of copper sulphate as an oxygen carrier, a few small globules of metallic mercury being added to 
the acid digestion mixture. Although the mercury salt is somewhat more efficient, it tenaciously retains ammonia, as

$$
\mathrm{H}_{2} \mathrm{~N} \cdot \mathrm{Hg} \cdot \mathrm{O} \cdot \mathrm{SO}_{2} \cdot \mathrm{O} \cdot \mathrm{Hg} \cdot \mathrm{NH}_{2},
$$

even in the presence of an excess of hot alkali, and it is therefore necessary to add also a large excess of sodium sulphide. This converts all the mercury into $\mathrm{HgS}$, which combines with $\mathrm{Na}_{2} \mathrm{~S}$ to form soluble $\mathrm{Hg}(\mathrm{SNa})_{2}$ and the ammonia is liberated.

4. The $\mathrm{K}_{2} \mathrm{SO}_{4}$ forms with the acid $\mathrm{KHSO}_{4}$, and this serves to raise the boiling point of the sulphuric acid; the higher temperature hastens the digestion.

5. The flask is provided with a long neck in order that the acid fumes, which would otherwise be lost, may condense and run back into the digestion mixture.

6. After the acid solution has been diluted, it is specifically lighter than the $\mathrm{NaOH}$ solution used; upon pouring the latter carefully down the neck of the inclined flask, it sinks to the bottom and leaves the surface of the liquid still acid, thus preventing the loss of ammonia at this stage of the procedure. The contents should not be mixed until after the flask has been tightly connected with the distilling head.

7. The granulated zinc is added in order to prevent bumping during the distillation; the zinc dissolves slowly in the alkaline solution, with the evolution of hydrogen. Fragments of pumice stone or of platinum are often used for the same purpose.

8. If nitrates are present in the sample (e.g. a fertilizer), and it is desired to determine the total nitrogen, the procedure may be modified as follows: Thoroughly wet the sample in the flask with $25 \mathrm{cc}$. of concentrated sulphuric acid, in which one gram of salicylic acid, $\left(\mathrm{C}_{6} \mathrm{H}_{4}<\mathrm{OOOH}\right)$ has previously been dissolved; this reacts with the nitric acid to form nitrosalicylic acid, $\mathrm{C}_{6} \mathrm{H}_{3}-\mathrm{OH}_{\mathrm{COOH}} \mathrm{NO}_{2}$ Next add slowly, with frequent shaking, to g. of powdered sodium thiosulphate, which reduces the nitrosalicylic acid to aminosalicylic acid, $\mathrm{C}_{6} \mathrm{H}_{3} \underset{\mathrm{COOH}}{\mathrm{OH}}$ - Now add $0.5 \mathrm{~g}$. of powdered copper sulphate and complete the determination as already described, but omitting the addition of the alkali sulphate (the solution contains $\mathrm{NaHSO}_{4}$ from the thiosulphate).

9. It is evident that ammonium salts may be analyzed for ammonia by simply distilling them with an excess of alkali, absorbing the ammonia in 
an excess of standard acid, etc. Moreover, nitric acid and nitrates may be quantitatively reduced to ammonia and determined in this way (see Part V, Problems 63, 64, and 89).

Certain other salts, as acetates, may be analyzed in an analogous manner by distillation with phosphoric acid in excess, the distillate being collected in standard alkali and the excess of the latter titrated, with the use of phenolphthalein. 


\section{B. METHODS OF OXIDATION AND REDUCTION}

Standard Solutions. In most oxidation and reduction processes the standard solution actually employed in the titration is one of an oxidizing reagent, though it is often an advantage also to have available a suitable standard reducing solution. Reducing substances are nearly always determined by direct titration, but in the case of oxidizing substances it is often more satisfactory to first add a reducing substance in excess, and then to titrate the excess of this reagent, or a product (as iodine) liberated from it by the oxidizing substance.

The most important reagents used in the preparation of standard oxidizing solutions are potassium permanganate, iodine, potassium dichromate, potassium bromate, and ferric chloride; and the reagents most useful for standard reducing solutions are ferrous ammonium sulphate, oxalic acid, sodium thiosulphate, sodium arsenite, and titanous chloride. Other substances are frequently used in volumetric processes for purposes of oxidation and reduction, but not often as standard solutions.

In the titration of a specific reducing substance, such as oxalic acid, it is of course necessary to employ a standard solution which will rapidly and completely oxidize the substance; in this instance, potassium permanganate. In general, the following pairs give satisfactory results : potassium permanganate and ferrous iron or oxalic acid; iodine and sodium thiosulphate or arsenite; potassium dichromate and ferrous iron;- ferric iron and titanous chloride.

Indicators. With respect to the indicators employed, potassium permanganate, owing to its intense coloring power, is its own indicator; the slightest excess is easily visible in otherwise colorless (or even in certain faintly colored) reaction mixtures. In the titration of ferrous iron with dichromate solutions, there is no really satisfactory indicator which can be added to the solution; but by means of potassium ferricyanide, as an outside indicator, it is possible to determine with great 
accuracy the end-point of the reaction. In the case of iodine, starch solution is employed as an indicator. The use of these indicators will be discussed under the respective processes.

\section{DICHROMATE PROCESSES}

Fundamental Principles. In the presence of acid, ferrousion is quantitatively oxidized in the cold to ferric-ion upon the addition of potassium dichromate solution. Since hydrochloric is the most efficient acid for dissolving the ores of iron, the titration is almost always carried out in the presence of this acid :

$6 \mathrm{FeCl}_{2}+\mathrm{K}_{2} \mathrm{Cr}_{2} \mathrm{O}_{7}+{ }_{4} \mathrm{HCl}=6 \mathrm{FeCl}_{3}+2 \mathrm{KCl}+2 \mathrm{CrCl}_{3}+{ }_{7} \mathrm{H}_{2} \mathrm{O}$.

1 The end-point of the titration is determined by means of a solution of potassium ferricyanide. As this point is approached, the iron solution is added dropwise to the indicator, on a white test-plate (or sheet of paper), and the mixture examined for a blue tint (due to the insoluble compound formed by the interaction of ferrous- and ferricyanide-ion). The indicator must of course be sufficiently dilute to be almost colorless, and must not contain any ferrocyanide. For the latter reason, since the solution is unstable, it must be prepared just before use.

THE PREPARATION AND STANDARDIZATION OF THE APPROXIMATELY TENTH-NORMAL DICHROMATE AND FERROUS IRON SOLUTIONS

Procedure. Dissolve $2.5 \mathrm{~g}$. of potassium dichromate in water, and dilute the solution to $500 \mathrm{cc}$; also dissolve $20 \mathrm{~g}$. of ferrous ammonium sulphate and $5 \mathrm{~g}$. of ammonium sulphate in water, add $5 \mathrm{cc}$. of concentrated sulphuric acid, and dilute to $500 \mathrm{cc}$. Thoroughly mix the solutions, allow them to come to the room temperature, and then fill a burette with each, with care to expel all air from the tips. Dissolve a cubic-millimeter fragment 
of pure potassium ferricyanide in $\mathbf{1 5} \mathrm{cc}$. of water, and with a glass rod transfer drops of this liquid to the white test-plate.

Measure out into an Erlenmeyer flask about $20 \mathrm{cc}$. of the iron solution, add IO-I 5 cc. of 6-normal hydrochloric acid and roo cc. of water, and then with shaking, run im 16-I8 cc. of the dichromate solution. Now, with the aid of a glass rod, transfer a drop of the solution to a drop of the indicator, being careful to wash the rod before returning it to the titration mixture. If a blue color at once shows up, add about $0.5 \mathrm{cc}$. more of the dichromate, and test again. As the blue tint shows up more and more faintly, add smaller and smaller amounts of the dichromate solution, and use larger drops of the titration mixture in the tests, until finally the bluish tinge fails to materialize in 30 seconds. Then, for the sake of comparison, make a new mixture beside the last test; if the two look exactly alike, the endpoint is indicated. While it is best not to overstep the endpoint, in this instance this can do no serious harm; it is only necessary to add more of the ferrous solution, and to proceed with the titration.

Repeat this operation until reliable checks are obtained, and from the (corrected) data calculate the value of the ferrous sulphate in terms of the dichromate solution; the duplicates should agree within two parts in one thousand.

Standardize the dichromate solution as follows: Weigh out three 0.I4-0.I6 g. portions of pure iron wire, free from rust, bundling them up before weighing (handling the wire with filter paper) so that they may easily be dropped into an Erlenmeyer flask. Treat each portion as follows: Heat $20 \mathrm{cc}$. of 6normal hydrochloric acid just to boiling in an Erlenmeyer flask, remove the flame, and drop in the wire; after solution is effected, boil gently for 2 minutes. While hot, add stannous chloride solution, drop by drop, until the mixture is decolorized; do not add more than 2 drops in excess. Cool, add Ioo cc. of cold water, and then pour in suddenly with shaking $15 \mathrm{cc}$. of mercuric chloride solution. After an interval of $\mathrm{I}$ minute, 
titrate the solution. It is best not to overstep the end-point; fewer burette readings will decrease the number of errors involved.

Notes. - I. The ionic changes due to oxidation and reduction are very marked in character, and the electrical charges on a resultant ion may even differ in sign from those on the initial ion; nevertheless, in the equations which represent these reactions, the algebraic sum of the charges on the one side is always equal to that of the charges on the other. In this case, for example, we have,

$$
6 \mathrm{Fe}^{++}+\mathrm{Cr}_{2} \mathrm{O}_{7}-+{ }_{4} \mathrm{H}^{+}=6 \mathrm{Fe}^{+++}+{ }_{2} \mathrm{Cr}^{+++}+{ }_{7} \mathrm{H}_{2} \mathrm{O} \text {. }
$$

2. An exactly tenth-normal solution of the dichromate may be prepared by dissolving $2.45 \mathrm{I} 7 \mathrm{~g}$. of the pure salt in water and accurately diluting the solution to $500 \mathrm{cc}$. The commercial salt may be purified by recrystallization, followed by drying to constant weight at $130^{\circ}$ (see Part I, D).

3. The presence of ammonium sulphate and sulphuric acid in the standard iron solution seems to increase the stability of the ferrous condition.

4. The iron content of the wire purchased for this standardization should be controlled by several gravimetric determinations. The wire will stay bright if kept in a desiccator over solid potassium hydroxide, but before use it should be examined for rust ; this may be removed by means of fine emery cloth.

5. The short boiling after solution is to expel any hydrocarbon that may still be present (the iron nearly always contains some carbon), which otherwise might reduce a little of the dichromate. This expulsion is even more important when the wire is used as a standard in connection with potassium permanganate.

6. In this titration, the iron to be determined must of course be wholly in the ferrous condition. It is common to reduce ferric iron to ferrous by means of stannous chloride, zinc and acid, or hydrogen sulphide, depending upon the conditions; but wherever permissible, it is most convenient to use stannous chloride. $\left(2 \mathrm{FeCl}_{3}+\mathrm{SnCl}_{2}=2 \mathrm{FeCl}_{2}+\mathrm{SnCl}_{4}\right.$.)

Since stannous chloride will readily reduce dichromate solution, the excess of this substance must be oxidized before the titration of the iron. This is done by means of mercuric chloride $\left(\mathrm{SnCl}_{2}+2 \mathrm{HgCl}_{2}=\mathrm{SnCl}_{4}\right.$ $+\mathrm{Hg}_{2} \mathrm{Cl}_{2}$ ), the chlorides of mercury being without influence on the titration. If present in any quantity, however, stannous chloride is likely to reduce the mercury partially to metal, and metallic mercury readily reduces dichromate solution. $\left(\mathrm{SnCl}_{2}+\mathrm{Hg}_{2} \mathrm{Cl}_{2}=\mathrm{SnCl}_{4}+2 \mathrm{Hg}\right.$. $)$ Therefore, the stannous chloride should be used in very slight excess, and to this end it is gradually added to the hot ferric solution, before the dilution of the latter. 
On the other hand, the excessive reduction of the mercury is less likely if the stannous chloride is cold and very dilute, and if the mercuric chloride is suddenly added in large excess. (The formation of a grayish precipitate is a sufficient indication that the solution should be discarded.)

7. The dichromate method for the determination of iron is capable of furnishing very accurate values; but, in addition to the usual precautions, the solution should be titrated promptly after the precipitation of the calomel (before aeration sets in), and the tests for the end-point should not be begun until that point is fairly imminent. In connection with the determination of this end-point, see also Note 2 under the gravimetric determination of iron. 


\section{THE DETERMINATION OF IRON IN SIDERITE}

Procedure. Weigh out $0.23-0.25$ g. portions of the powdered ore into Erlenmeyer flasks, moisten the samples with water, and add to each $20 \mathrm{cc}$. of 6-normal hydrochloric acid and about $0.2 \mathrm{~g}$. of potassium chlorate. Heat not quite to boiling as long as there is solvent action, and to the hot solution add stannous chloride solution, drop by drop, not more than 2 drops in excess. Cool, add $100 \mathrm{cc}$. of water, shake, and then pour in $15 \mathrm{cc}$. of mercuric chloride solution. After an interval of $\mathrm{I}$ minute, titrate the solution. Report the percentage of iron in the ore.

Notes. - I. Siderite (native ferrous carbonate) is an iron ore plentiful in England. It is apt to contain organic matter, and, in order to destroy this, potassium chlorate is added during its solution.

2. Other ores can of course be analyzed by this method. Since most of them contain ferric iron, or, since in the case of ferrous ores the iron is generally oxidized during the preparation of the solution, the amount of stannous chloride to be added is greater than that required in the standardization against iron wire. In no case, however, should the excess used exceed 2 drops.

3. For a more general method of dissolving iron ores, see the determination of iron by means of potassium permanganate, and also Note I under that method. 


\section{PERMANGANATE PROCESSES}

Fundamental Principles. In acid solution, potassium permanganate readily oxidizes ferrous-ion to ferric, at the ordinary temperature. Also, starting at $80-90^{\circ}$, it reacts quantitatively with oxalic acid, which it oxidizes to carbonic acid. Though in reality the reactions are not so simple, the quantitative relationships are accurately represented by the following equations :

Io $\mathrm{FeSO}_{4}+2 \mathrm{KMnO}_{4}+9 \mathrm{H}_{2} \mathrm{SO}_{4}={ }_{5} \mathrm{Fe}_{2}\left(\mathrm{SO}_{4}\right)_{3}+2 \mathrm{KHSO}_{4}$ $+2 \mathrm{MnSO}_{4}+8 \mathrm{H}_{2} \mathrm{O}$; and $5 \mathrm{H}_{2} \mathrm{C}_{2} \mathrm{O}_{4}+2 \mathrm{KMnO}_{4}+4 \mathrm{H}_{2} \mathrm{SO}_{4}=2 \mathrm{KHSO}_{4}+2 \mathrm{MnSO}_{4}$

Or, more simply expressed, + $\mathrm{IO} \mathrm{CO}_{2}+8 \mathrm{H}_{2} \mathrm{O}$.

$$
{ }_{5} \mathrm{Fe}^{++}+\mathrm{MnO}_{4}^{-}+8 \mathrm{H}^{+}={ }_{5} \mathrm{Fe}^{+++}+\mathrm{Mn}^{++}+{ }_{4} \mathrm{H}_{2} \mathrm{O} \text {; }
$$

and $\quad{ }_{5} \mathrm{C}_{2} \mathrm{O}_{4}^{--}+2 \mathrm{MnO}_{4}^{-}+\mathrm{I} 6 \mathrm{H}^{+}=2 \mathrm{Mn}^{++}+$Io $\mathrm{CO}_{2}+8 \mathrm{H}_{2} \mathrm{O}$.

In a hot neutral or faintly acid solution, in the presence of zinc salts, potassium permanganate oxidizes manganous salts quantitatively in the sense of the equation,

$$
3 \mathrm{Mn}^{++}+2 \mathrm{MnO}_{4}^{-}+2 \mathrm{H}_{2} \mathrm{O}={ }_{4} \mathrm{H}^{+}+5 \mathrm{MnO}_{2} \text {. }
$$

From these equations it is readily seen that for use in acid solution the normal weight of the salt is one fifth of a mol, or 3r.6I g., while for use in the determination of manganese the normal weight is one third of a mol, or $52.68 \mathrm{~g}$. It is not customary, however, in volumetric analysis, to employ solutions of permanganate of greater than tenth-normal concentration.

In addition to the above, potassium permanganate is capable of oxidizing stannous, cuprous, and mercurous salts, antimonius, arsenious, nitrous, and sulphurous acids, hydrogen sulphide, ferrocyanides, and many other substances.

Furthermore, as a less desirable feature, the permanganate is capable under certain conditions of oxidizing free hydrochloric acid, with the liberation of chlorine. This action, though almost imperceptible with cold dilute hydrochloric acid, is vigor- 
ously catalyzed by ferrous iron. With suitable modifications, however, it is possible to obtain very exact results in the presence of hydrochloric aid, even in the titration of iron; but, other things being equal, in acid solution, it is preferable to carry out permanganate titrations in the absence of chlorides.

Even at tenth-normal concentration, potassium permanganate solution is so deeply colored that the lower line of the meniscus is not clearly visible in an ordinary burette; readings must therefore be made from the upper edge. This disadvantage, however, is more than offset by the fact that the presence of a single drop in excess, in an otherwise colorless solution, may be recognized with great ease; as its own indicator, it leaves nothing to be desired.

The permanganate solution should not be placed in burettes with rubber connections; it is more or less rapidly reduced by most organic substances.

\section{THE PREPARATION AND STANDARDIZATION OF AN AP- PROXIMATELY TENTH-NORMAL SOLUTION OF POTAS- SIUM PERMANGANATE}

Procedure. Dissolve $3.25 \mathrm{~g}$. of the salt in $250 \mathrm{cc}$. of warm water, allow to cool, dilute to I liter, and mix thoroughly. The value of this solution is apt to change slowly, especially at first, and for this reason the solution should be allowed to stand for several days, and then filtered through a layer of asbestos to remove the precipitate of hydrated manganese dioxide. After thorough mixing, it is then ready for standardization. The solution should be preserved in glass-stoppered bottles, and should be protected from heat and light. Thus prepared and preserved, it will retain its oxidizing value for months. The solution is said to be still more stable if it is made very slightly alkaline with potassium hydroxide (before standardization, of course).

Weigh out accurately into $700 \mathrm{cc}$. Erlenmeyer flasks several $0.12-0.14$ g. samples of pure sodium oxalate, previously dried 
at Iro-r $20^{\circ}$; dissolve each sample in $250 \mathrm{cc}$. of hot water (80$90^{\circ}$ ), with the addition of $30 \mathrm{cc}$. of 6 -normal sulphuric acid, and titrate at once with the permanganate solution. At first, the permanganate should be added drop by drop, with shaking after each addition until the color disappears. After several drops have been added, the solution may be run in slowly (IO-I 2 cc. per minute) with continuous shaking. Toward the end of the titration, particular care must be taken to allow the color due to each drop to disappear before the addition of the next, in order to avoid passing the end-point. Titrate to the first permanent pink. The temperature at the end of the titration must not be below $60^{\circ}$.

From the data obtained, calculate the normality factor of the solution. Duplicate values should check within two parts in one thousand.

Notes. - I. It is not satisfactory to prepare a standard solution by directly weighing out the calculated quantity of potassium permanganate, even after the latter has been purified by recrystallization. The solution should be prepared as described in the procedure, and then standardized against iron wire or sodium oxalate. While ferrous ammonium sulphate, oxalic acid, potassium tetroxalate, and other compounds might be used as standards, iron wire and sodium oxalate are readily obtainable in a sufficiently pure condition, and being non-hygroscopic and free from water of crystallization, their composition is less subject to change.

2. Upon treating a given weight of pure sodium oxalate with an excess of sulphuric acid, the corresponding weight of oxalic acid is set free; so that the use of this salt as a standard merely enables us easily to measure out a specific amount of oxalic acid. The oxidation of the oxalic acid by the permanganate is at first slow, and the permanganate should be added dropwise, with full time for decolorization between successive drops. After a certain small amount of manganous sulphate has been produced in the solution, however, the speed of the reaction is very greatly increased (by the catalytic action of this substance) and the permanganate may be run in much faster. (See R. S. McBride: Jour. Amer. Chem. Soc., vol. 34, p. $4 \mathrm{I} 5$ ( 1912$)$.) 


\section{THE DETERMINATION OF IRON IN HEMATITE}

Principles. One of the most accurate methods for the determination of iron is based upon the oxidation of a chloride-free ferrous sulphate solution, in the presence of sulphuric acid, with potassium permanganate. Under these conditions, ferrous iron is oxidized and permanganate is reduced, according to the equation:

$$
\mathrm{MnO}_{4}^{-}+5 \mathrm{Fe}^{++}+8 \mathrm{H}^{+}=\mathrm{Mn}^{++}+5 \mathrm{Fe}^{+++}+4 \mathrm{H}_{2} \mathrm{O} \text {. }
$$

But if chlorides are present, some of the permanganate will be reduced by these, with the liberation (and partial escape) of chlorine, and the results will be somewhat high:

$$
2 \mathrm{MnO}_{4}^{-}+\mathrm{r}^{6} \mathrm{H}^{+}+\text {ro } \mathrm{Cl}^{-}=2 \mathrm{Mn}^{++}+8 \mathrm{H}_{2} \mathrm{O}+5 \mathrm{Cl}_{2} \text {. }
$$

Upon the addition of the permanganate to a cold, dilute solution of hydrochloric acid alone, or to one containing ferric iron, no chlorine is evolved; ferrous iron, therefore, seems to accelerate this reaction by catalysis.

Nevertheless, since in dissolving iron ores it is nearly always necessary to use strong hydrochloric acid, to which it is often well to add a little stannous chloride, and since stannous chloride is a most convenient reagent for the reduction of ferric iron to the ferrous condition, it is desirable, if possible, to carry out the titration in the presence of fairly large quantities of chlorides.

Now it has been shown that if, when chlorides are present, a small quantity of manganous salt is added to the solution, the ferrous iron alone is oxidized, and that accurate titrations can be performed (Zimmermann). But the end-point is somewhat indistinct, owing to the yellow tint of the ferric chloride produced. This difficulty can be overcome by the addition of phosphoric and sulphuric acids (Reinhardt), which have recently been shown to combine with ferric iron to form colorless complexes such as $\mathrm{H}\left[\mathrm{Fe}\left(\mathrm{SO}_{4}\right)_{2}\right], \mathrm{H}_{3}\left[\mathrm{Fe}\left(\mathrm{PO}_{4}\right)_{2}\right]$, and $\mathrm{H}_{6}\left[\mathrm{Fe}\left(\mathrm{PO}_{4}\right)_{3}\right]$ (Weinland and Ensgraber, Zeitschrift für anorganische Chemie, 
vol. 84, p. 349); the large excesses of these acids repress the dissociation of these complexes and insure a colorless solution.

Procedure. Weigh out three samples of the finely ground ore, of about $0.25 \mathrm{~g}$. each, into Ioo cc. beakers. To each sample add ${ }_{5} \mathrm{cc}$. of 6-normal hydrochloric acid and $2 \mathrm{cc}$. of stannous chloride solution, and gently heat the covered beakers for ro-r 5 minutes, until nothing other than a small, white, sandy residue remains undissolved. If the hot solution is at all yellow, discharge this color by adding stannous chloride solution, one drop at a time, with stirring; avoid an excess of more than two drops. If, however, after the heating, the solution is colorless, stannous chloride is present in unknown excess, and must be oxidized by adding permanganate solution (not to be counted, of course, in the volume required for the titration) drop by drop with stirring, until the yellow color due to ferric iron appears; discharge this color as above directed, with stannous chloride solution, one drop in excess.

After cooling, dilute the colorless solution with $50 \mathrm{cc}$. of cold water, and transfer, with stirring, to a 700-cc. beaker containing ro cc. of mercuric chloride and $50 \mathrm{cc}$. of water. (If, instead of a white precipitate of calomel, a gray precipitate of mercury is formed at this point, the solution must be discarded.) Dilute the mixture with cold water to about $500 \mathrm{cc}$, add 8-10 cc. of the Zimmermann-Reinhardt solution, ${ }^{1}$ and titrate at once with the standard permanganate solution. Add the permanganate slowly, with constant stirring, finally in single drops, until the pink color flashes throughout the solution and persists for $1^{5-20}$ seconds; do not pass the end-point. Report the percentage of iron in the ore.

Notes. - r. Many iron ores are not completely decomposed by hydrochloric acid, the insoluble residue containing more or less iron, as silicate, titaniferous iron, etc. Unless iron is known to be absent in the insoluble

1 Made by dissolving $67 \mathrm{~g}$. of $\mathrm{MnSO}_{4} \cdot{ }_{4} \mathrm{H}_{2} \mathrm{O}$ in $500 \mathrm{cc}$. of water, adding $\mathrm{I}_{3} 8 \mathrm{cc}$. of phosphoric acid (sp. gr., I.7) and r30 cc. of sulphuric acid (sp. gr., r.84), and diluting with water to one liter. 
residue, the finely ground sample should be digested on the hot plate with ro cc. of hydrochloric acid until the residue is white, or until there appears to be no further action; if the ore contains carbonaceous matter, a little potassium chlorate should be added. Finally evaporate to dryness, extract with $5 \mathrm{cc}$. of hydrochloric acid, dilute with Io $\mathrm{cc}$. of water, allow to settle, and decant the clear liquid through a small filter, transferring the residue to the filter and washing with as little cold water as possible. Ignite the filter and residue in a small platinum crucible, allow to cool, and add 20-30 drops of sulphuric acid and twice as much hydrofluoric acid. Heat carefully, and, if the residue is dissolved, evaporate to white fumes, allow to cool, dissolve in water, and add to the solution at first obtained. If, however, this treatment fails to decompose the residue, drive off most of the sulphuric acid, add $0.5-0.6 \mathrm{~g}$. of potassium bisulphate, and heat gradually until the bisulphate is quite liquid and fumes of sulphuric acid are given off whenever the lid of the crucible is raised. When the black specks have disappeared, allow the crucible to cool and dissolve the salt in the crucible with hot water and a few drops of hydrochloric acid.

In case ferric iron has been dissolved in hydrochloric acid in contact with platinum, the solution should be oxidized with bromine water and the iron precipitated with ammonia; i.e. if it is desired to use stannous chloride in the reduction. The ferric hydroxide can then be redissolved (after washing it with hot water) in hydrochloric acid and reduced. Otherwise the iron solution will contain a small quantity of platinum, ${ }_{4} \mathrm{FeCl}_{3}+2 \mathrm{HCl}+\mathrm{Pt}$ $=4 \mathrm{FeCl}_{2}+\mathrm{H}_{2} \mathrm{PtCl}_{6}$, which gives a characteristic ferric-iron color with stannous chloride, and prevents the recognition of the point at which the iron is reduced.

2. Three samples should be taken, in order that one may be used for a rapid preliminary titration. Having ascertained in a rough manner the iron content of the sample, the final titrations are greaty facilitated.

3. Stannous chloride is a great help in the solution of many ores containing ferric iron. Apparently the diffrcultly soluble particles of hematite are continuously reduced at the surface to ferrous oxide, which is much more readily dissolved by the acid.

4. Some common agents for the reduction of ferric iron are zinc, sulphurous acid, and hydrogen sulphide; stannous chloride is excluded unless the titration is to be made by the Zimmermann-Reinhardt method. In that case it should be carefully added, in very slight excess, to the hot, concentrated, acid solution (cf. the standardization of dichromate solution, Note 6).

5. Soluble salts of mercurous mercury are readily oxidized by potassium permanganate in acid solution. Mercurous chloride, however, is exceedingly insoluble, and, provided only a very small quantity is suspended in 
the solution, its action is so slow that the end-point of the titration can be accurately fixed. The pink color which flashes throughout the solution at the end of the titration is, however, not permanent, and for that reason the time-limit set should be closely observed. For the greatest accuracy, the permanganate should of course be standardized, under exactly the same conditions, against a known quantity of metallic iron. But the error due to the use of a solution standardized against sodium oxalate is for most purposes negligible.

6. For a rapid method for the reduction of ferric iron by means of zinc, see Notes I and 2 under the Determination of Phosphorus in Steel. It should be noted that titanium is also reduced by zinc, but not by the other agents mentioned; with the use of zinc, therefore the presence of titanium would lead to high results. 


\section{THE DETERMINATION OF CALCIUM IN LIMESTONE}

Procedure. Instead of igniting the precipitate of calcium oxalate, obtained from the limestone by double precipitation according to the procedure described in Part II, and weighing it as calcium oxide, the calcium may be determined volumetrically as follows: Wash the reprecipitated calcium oxalate by decantation, keeping it as far as possible in the precipitation vessel, and decompose this precipitate by slowly pouring through the filter at least six $5 \mathrm{cc}$. portions of hot, 3-normal sulphuric acid, washing afterwards with hot water, and receiving the acid filtrate and washings in the beaker containing the bulk of the precipitate. Dilute this mixture to $100 \mathrm{cc}$. and warm gently, with stirring, to completely decompose the calcium oxalate. Allow the mixture to cool, transfer it quantitatively to a 250 -cc. measuring flask, and dilute to the mark with water, finally mixing the solution by pouring it into a clean $d r y$ beaker and back into the flask.

Measure out by means of a pipette $50.00 \mathrm{cc}$. portions of this solution, add to each $30 \mathrm{cc}$. of 6-normal sulphuric acid, dilute to $300 \mathrm{cc}$., heat to $90^{\circ}$, and titrate as already described with the standard permanganate solution. Remembering that only one fifth of the sample was used in each titration, calculate the percentage of $\mathrm{CaO}$ in the limestone.

Note. - The reactions involved in the volumetric determination of calcium are:

$$
\begin{aligned}
\mathrm{CaC}_{2} \mathrm{O}_{4}+\mathrm{H}_{2} \mathrm{SO}_{4} & =\mathrm{CaSO}_{4}+\mathrm{H}_{2} \mathrm{C}_{2} \mathrm{O}_{4} ; \text { and } 5 \mathrm{H}_{2} \mathrm{C}_{2} \mathrm{O}_{4}+2 \mathrm{KMnO}_{4} \\
+{ }_{4} \mathrm{H}_{2} \mathrm{SO}_{4} & =2 \mathrm{KHSO}_{4}+2 \mathrm{MnSO}_{4}+\mathrm{Io}_{2} \mathrm{CO}_{2}+8 \mathrm{H}_{2} \mathrm{O} .
\end{aligned}
$$

Therefore, the normal weight of calcium oxide in this case is one half of a mol; or $0.1 N$ permanganate solution has a calcium oxide value of $0.00280 \mathrm{~g}$. per cubic centimeter. 
THE DETERMINATION OF THE $\mathrm{MNO}_{2}$-VALUE OF PYROLUSITE

Procedure. Weigh out two portions of the finely ground mineral, of about $0.3 \mathrm{~g}$. each, into $700 \mathrm{cc}$. Erlenmeyer flasks. Calculate the weight of ferrous ammonium sulphate, $\mathrm{Fe}\left(\mathrm{NH}_{4} \mathrm{SO}_{4}\right)_{2} .6 \mathrm{H}_{2} \mathrm{O}$, required to react with each sample, on the basis that it is pure manganese dioxide:

$$
2 \mathrm{FeSO}_{4}+\mathrm{MnO}_{2}+2 \mathrm{H}_{2} \mathrm{SO}_{4}=\mathrm{Fe}_{2}\left(\mathrm{SO}_{4}\right)_{3}+\mathrm{MnSO}_{4}+2 \mathrm{H}_{2} \mathrm{O} \text {, }
$$

and weigh out accurately portions of the pure salt $0.15^{-0.20} \mathrm{~g}$. in excess of the calculated amounts, into the corresponding flasks. Add to each flask $50 \mathrm{cc}$. of water and $50 \mathrm{cc}$. of 6-normal sulphuric acid, cover the flasks, and heat to boiling until the action is complete. Finally, dilute to about $300 \mathrm{cc}$., and promptly titrate the excess of ferrous iron with the standard permanganate solution. From the data obtained, calculate the percentage of $\mathrm{MnO}_{2}$ in the sample.

Notes. - I. In order that solution shall take place without difficulty, the mineral should be ground fine enough to wholly pass through a roo-mesh sieve. In that case, a moderate excess of ferrous iron will insure rapid

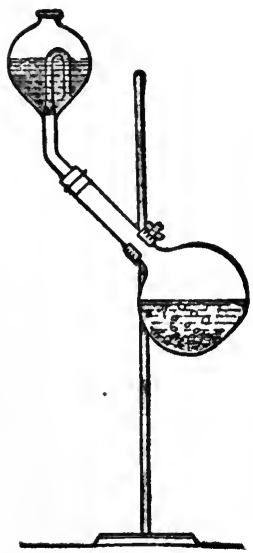
solution, provided the mixture is not diluted before solvent action has ceased.

2. A solution of iron wire in sulphuric acid may be substituted for the ferrous ammonium sulphate, but in that case there is more danger of the partial oxidation of the iron by the air. For example, if iron wire is used, it should be dissolved in sulphuric acid out of contact with air, and the air should not have access to the solution during cooling. This is best accomplished by means of a Contat-Göckel valve, which consists of.a glass bulb with an inner siphon, as shown in the figure. In the bulb is placed a cold saturated solution of sodium bicarbonate, through which the hydrogen (and steam) evolved in the flask bubbles. After all the iron has been dissolved, the liquid is boiled for a few minutes longer, and the flame is removed. As the flask cools off, small portions of the bicarbonate are at intervals sucked into the flask and decomposed by the acid with the 
evolution of carbon dioxide, whereby the entrance of more bicarbonate solution is prevented.

For other methods of performing this analysis, see Part V, Problems 23, 73, and 74. According to O. L. Barnebey (J. Ind. Eng. Chem., vol. 9, p. 96I (I9I7)), the use of oxalic acid in place of the ferrous salt yields less reliable results.

3. With the substitution of very dilute nitric acid for sulphuric acid in the above procedure, the method may be used to determine the $\mathrm{PbO}_{2}$ value of red lead, or minium, $\mathrm{Pb}_{3} \mathrm{O}_{4}$, and of lead peroxide, $\mathrm{PbO}_{2}$. Of these substances, samples of $\mathrm{I} .0$ and $0.8 \mathrm{~g}$., respectively, should be taken when 30-cc. burettes are used. It is better, however, to make use of an iodometric method. 


\section{THE DETERMINATION OF PHOSPHORUS IN STEEL}

Principle. The molybdic anhydride contained in ammonium phosphomolybdate, $\left(\mathrm{NH}_{4}\right)_{3} \mathrm{PO}_{4}$. I2 $\mathrm{MoO}_{3}$, may be reduced by zinc in the presence of sulphuric acid, from $\mathrm{MoO}_{3}$ to $\mathrm{Mo}_{2} \mathrm{O}_{3}$; but molybdenum in the latter condition is not stable in the presence of air. If, however, the acidified molybdate solution is passed through a Jones reductor (see below) directly into a solution of ferric sulphate, the sensitive molybdate compound is oxidized by the ferric salt with the formation of an equivalent amount of ferrous sulphate, less sensitive to the atmospheric action. The molybdenum solution is green as it leaves the reductor, but upon mixing with the ferric salt the green color disappears; if phosphoric acid is added, the color due to the presence of ferric iron is destroyed. The decolorized solution is titrated while still hot with tenth-normal permanganate solution, of which the quantity necessary corresponds to the equation,

$$
5 \mathrm{Mo}_{2} \mathrm{O}_{3}+6 \mathrm{KMnO}_{4}=3 \mathrm{~K}_{2} \mathrm{O}+6 \mathrm{MnO} \text { + } 10 \mathrm{MoO}_{3} \text {. }
$$

From this it may be seen that $5 \mathrm{P} \approx 30 \mathrm{Mo}_{2} \mathrm{O}_{3} \approx 36 \mathrm{KMnO}_{4} \approx 90 \mathrm{O}$, or $\mathrm{P} \approx \mathrm{I} 8 \mathrm{O}$; one cubic centimeter of o.I $N$ permanganate solution represents, therefore, $0.0862 \mathrm{mg}$. of phosphorus.

Procedure. Weigh out two samples of steel drillings, each sufficient to contain $1.7-2.0 \mathrm{mg}$. of phosphorus, into 250-cc. Erlenmeyer flasks. Add to each a mixture of $25 \mathrm{cc}$. of nitric acid (sp. gr., r.42) and 75 cc. of water. Suspend in the neck of each flask a small funnel and heat until, after complete solution, the oxides of nitrogen have been expelled. Dissolve $0.3^{-}$ 0.4 g. of $\mathrm{KMnO}_{4}$ crystals in ro cc. of hot water, add one half of this solution to the contents of each flask, and boil until the permanganate color has disappeared. Remove the flame, add sulphurous acid or ammonium bisulphite solution, a few drops in excess, to dissolve the precipitated oxides of manganese, boil out the excess of sulphur dioxide, and filter the solution; re- 
ceiving the filtrate in a similar flask. Add ammonia to the solution with stirring until a permanent precipitate just begins to form, and then add nitric acid drop by drop to clear up the solution. Finally, at a temperature of $40^{\circ}$, add $40 \mathrm{cc}$. of molybdate solution, close the flask with a rubber stopper, and shake vigorously for five minutes; allow the precipitate to settle. (At this point, prepare the Jones reductor for use, as described in Note 2.)

Now filter the solution, keeping the precipitate as far as possible in the flask, and wash by decantation with a solution of ammonium sulphate acidified with sulphuric acid ${ }^{1}$ until the washings give no test for molybdenum with ammonium sulphide and hydrochloric acid. Dissolve the precipitate by pouring through the filter a mixture of $5 \mathrm{cc}$. of 6-normal ammonia and $20 \mathrm{cc}$. of water, and collecting the filtrate and washings in the precipitation flask. Acidify the solution, which should have a volume of about $60 \mathrm{cc}$., with Io cc. of sulphuric acid (sp. gr., r.84) and promptly pass the acidified solution, before it has a chance to cool off, through the reductor into the receiver (collecting the liquid beneath the surface of roo cc. of a solution containing $25 \mathrm{~g}$. of ferric alum and $40 \mathrm{cc}$. of sirupy phosphoric acid, sp. gr., I.7, per liter), preceded by roo cc. of hot water and followed by $200 \mathrm{cc}$. of hot dilute sulphuric acid $(\mathrm{r}: 40)$ and by roo cc. of hot water. See that no air enters the reductor during this entire operation. Titrate the reduced solution at once with tenth-normal permanganate, and calculate the percentage of phosphorus in the steel on the assumption that the yellow precipitate contains phosphorus and molybdenum in the proportion indicated by the formula $\left(\mathrm{NH}_{4}\right)_{3} \mathrm{PO}_{4} \cdot \mathrm{I}_{2} \mathrm{MoO}_{3}$.

Notes. - $\mathrm{r}$. The Jones reductor, which also is useful in the reduction of ferric iron for titration, is essentially a column of amalgamated zinc, through which the solution is passed for reduction. It is assembled as shown in the figure on p. 142 . The tube $A$ is of about $18 \mathrm{~mm}$. inside

${ }^{1}$ Made by mixing 15 cc. of ammonia (sp. gr., 0.90 ) and 25 cc. of sulphuric acid (sp. gr., r.84) with one liter of water. 
diameter, and (for this reduction) $400 \mathrm{~mm}$. long; the small extension tube is of $6 \mathrm{~mm}$. inside diameter and extends downwards $300 \mathrm{~mm}$. from the stopcock. The outlet of the tube $A$ is covered with a layer of glass beads; these are surmounted by a small plug of glass wool ; and upon this is placed a layer of asbestos felt, not exceeding $\mathbf{I ~ m m}$. in thickness. Amalgamated zinc is then added to within $50 \mathrm{~mm}$. of the top of the tube, and this may be

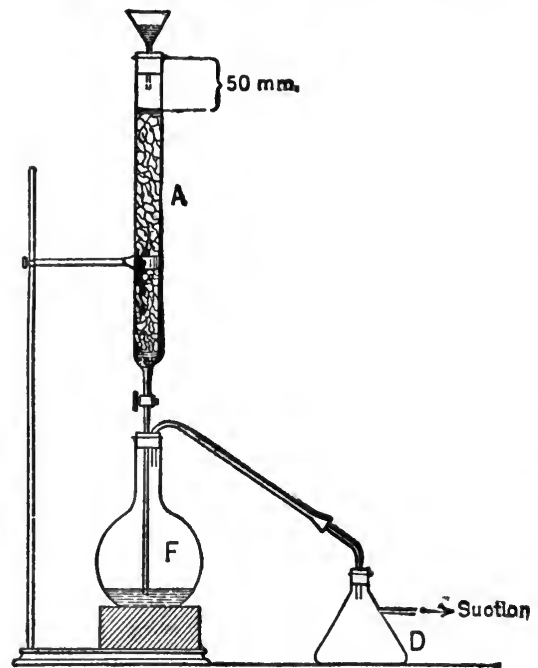
covered with glass wool and asbestos felt, to retain any solid matter. The reductor is connected as shown with the suction flask $F$, which in turn is connected with the safety bottle $D$, to avoid the intake of any contamination from the suction apparatus.

The amalgamated zinc is prepared by dissolving $5^{-6} \mathrm{~g}$. of mercuric chloride in $250 \mathrm{cc}$. of water, in a I liter bottle, with the addition of 5-10 cc. of 6-normal hydrochloric acid, adding to this solution $500 \mathrm{~g}$. of (I $8 \mathrm{mesh}$ ) granulated zinc, and shaking vigorously for at least a minute; the liquid is then decanted, and the zinc thoroughly washed with water.

2. For use, the reductor is connected as shown with the suction apparatus; and, with the stopcock almost closed, it is filled with warm sulphuric acid ( $25 \mathrm{cc}$. of the concentrated acid in I liter); the stopcock is then opened to allow the acid to run through slowly. Acid is poured in until $200-300 \mathrm{cc}$. have passed through, and then with liquid still in the funnel, the cock is closed. (In using this apparatus, see that no air enters the reductor; otherwise hydrogen peroxide may be formed from oxygen and nascent hydrogen and vitiate the results.) The first filtrate is rejected, $200 \mathrm{cc}$. more of the warm acid are passed through, followed by $100 \mathrm{cc}$. of warm water, and to this liquid $(300 \mathrm{cc}$.) the standard permanganate solution is added, one drop at a time, from a burette, in order to determine the volume required to color the acid solution alone. This amount (required by impurities from the zinc) must be subtracted from the volume required in the subsequent titration.

3. Upon dissolving the steel in nitric acid of the strength indicated, the phosphorus is oxidized, and none of it is lost by evolution as phosphine. 
The permanganate is subsequently added in order to insure the complete oxidation of carbonaceous matter and of the phosphorus to phosphoric acid.

4. The higher oxides of manganese, as $\mathrm{MnO}_{2}$, are not soluble in nitric acid. Upon the addition of a reducing agent, however, such as hydrogen peroxide or sulphurous acid, their solution is effected:

$$
\mathrm{MnO}_{2}+\mathrm{H}_{2} \mathrm{SO}_{3}=\mathrm{MnO}+\mathrm{H}_{2} \mathrm{SO}_{4}=\mathrm{MnSO}_{4}+\mathrm{H}_{2} \mathrm{O} \text {. }
$$

5. In connection with the precipitation of phosphoric acid as ammonium phosphomolybdate, the student should consult the notes under the Determination of Phosphoric Anhydride.

6. Since the molybdenum in the precipitate prepared from one gram of a steel containing $0.15 \%$ of phosphorus would require by this method r 7.44 cc. of o.r-normal permanganate solution, it is readily seen that the process is a rapid one for arriving at very accurate results. This is especially true if the permanganate has been standardized under the same conditions against a steel of accurately known phosphorus content; in such a case, it would be unnecessary to correct for the small amount of iron extracted from the (impure) amalgamated zinc, since this would be the same in both standardization and analysis.

7. It scarcely needs to be pointed out that the method is not suitable for determining phosphorus or phosphoric acid in substances containing them in large amount. This would require for titration relatively enormous quantities of permanganate solution, and, what is still worse, it would be practically impossible to completely reduce the molybdenum. If small aliquot portions were taken for reduction and titration, any error of measurement would be multiplied by a very large factor in the calculation of the result.

8. The following method is suitable for the volumetric determination of phosphorus or phosphoric acid when these are present in larger amounts. The phosphorus or phosphoric acid is converted into ammonium phosphomolybdate; this, after washing with $\mathrm{KNO}_{3}$ solution, is dissolved in an excess of standard sodium hydroxide solution; and the resulting solution is titrated with standard nitric acid, with phenolphthalein as an indicator. Needless to say, the sodium hydroxide should be standardized under identical conditions against a sample of accurately known phosphorus content. 


\section{THE DETERMINATION OF MANGANESE IN AN ORE}

Fundamental Principles. When potassium permanganate is added to a hot, neutral, or very faintly acid solution of manganese sulphate an action takes place in which the manganous oxide of the sulphate is oxidized at the expense of the anhydride of the permanganate, with the precipitation of hydrated intermediate oxides in varying proportions. These are manganous acid, $\mathrm{MnO}(\mathrm{OH})_{2}$, and hydrated salts of manganous acid. The essential changes in the state of oxidation may be represented as follows :

$$
\begin{aligned}
\mathrm{Mn}_{2} \mathrm{O}_{7}+3 \mathrm{MnO} & ={ }_{5} \mathrm{MnO}_{2} ; \\
\mathrm{Mn}_{2} \mathrm{O}_{7}+8 \mathrm{MnO} & ={ }_{5}\left(\mathrm{MnO}_{2} \cdot \mathrm{MnO}\right) ; \\
\mathrm{Mn}_{2} \mathrm{O}_{7}+\mathrm{I}_{3} \mathrm{MnO} & ={ }_{5}\left(\mathrm{MnO}_{2} \cdot 2 \mathrm{MnO}\right) .
\end{aligned}
$$

It is clear, then, that in this form the action cannot furnish the basis for a satisfactory volumetric method.

It has been found, however, that under suitable conditions, in the presence of zinc-ion, a hydrated manganite of zinc is precipitated, which, while variable in composition, contains all the manganese in the quadrivalent condition. Thus regulated, the reaction furnishes a valuable means for the determination of manganese (Volhard's Method). Although the composition of the precipitate varies, the course of the reaction is typically represented by the following equation:

${ }_{4} \mathrm{KMnO}_{4}+{ }_{5} \mathrm{ZnSO}_{4}+6 \mathrm{MnSO}_{4}+\mathrm{I}_{4} \mathrm{H}_{2} \mathrm{O}={ }_{2} \mathrm{~K}_{2} \mathrm{SO}_{4}+9 \mathrm{H}_{2} \mathrm{SO}_{4}$

or, more simply,

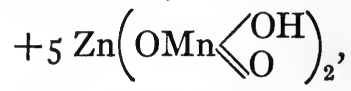

$$
2 \mathrm{MnO}_{4}^{-}+3 \mathrm{Mn}^{++}+2 \mathrm{H}_{2} \mathrm{O}=4 \mathrm{H}^{+}+5 \mathrm{MnO}_{2} \text {. }
$$

Procedure. Weigh out into a 500-cc. Erlenmeyer flask a sufficient quantity of the very finely ground ore to contain about $0.20 \mathrm{~g}$. of manganese; add $3 \mathrm{~g}$. of potassium chlorate and $20 \mathrm{cc}$. of I2-normal hydrochloric acid, and boil until the ore is completely decomposed and the chlorine expelled. Dilute with water to about $50 \mathrm{cc}$; quantitatively transfer the cold solution 
to a Ioo-cc. measuring flask; dilute to the mark with water; and mix thoroughly by pouring the contents of the flask into a clean, dry beaker, and back into the flask.

Now, from a burette or pipette, measure into 500-cc. Erlenmeyer flasks four $20.00 \mathrm{cc}$. portions of this solution, and treat each as follows: Dilute with water to roo cc., heat, and to the acid solution add with shaking an aqueous suspension of zinc oxide, ${ }^{1}$ in small portions, until the iron is completely precipitated as ferric hydroxide; this point may be recognized by the sudden coagulation of the precipitate, upon shaking, and the decolorization of the brownish colored solution. The precipitate should not be light yellow, but should have the characteristic brownish red color of ferric hydroxide, and the least possible excess of zinc oxide should be used. (Should the ore contain a quantity of iron insufficiently in excess of that required by any phosphoric and arsenic acids present, then $5 \mathrm{cc}$. of a solution containing $20 \mathrm{~g}$. of ferric chloride per liter should be added before the precipitation with zinc oxide.) If too much zinc oxide is added, the solution will be milky; in that case very dilute hydrochloric acid should be added drop by drop to the hot solution until the supernatant liquid just becomes clear.

Finally dilute the solutions to $300 \mathrm{cc}$. and, at $80^{\circ}$, treat them successively as follows: Run into the first solution the standard permanganate in $5 \mathrm{cc}$. portions, until after continued shaking the liquid retains a permanent pink tinge, - say after the addition of the fifth portion (i.e. $25 \mathrm{cc}$.) ; into the second solution run $5 \mathrm{cc}$. less permanganate than the volume previously used

1 Dissolve roo g. of crystallized zinc sulphate in $300 \mathrm{cc}$. of hot water, and with stirring cautiously add to the clear solution a few drops of a solution made by dissolving $25-27 \mathrm{~g}$. of pure sodium hydroxide in $150 \mathrm{cc}$. of water, until the zinc solution remains distinctly turbid; then add a little bromine water, heat, and filter. To the filtrate add the bulk of the sodium hydroxide solution, and stir. Rinse the mixture into a one-liter bottle, and fill the latter with water. The mixture should be well shaken when used. (This suspension should not react alkaline with phenolphthalein, and a ro cc. portion of the mixture, when cleared up with sulphuric acid, diluted to roo cc., and treated with one drop of $0.1 \mathrm{~N} \mathrm{KMnO}_{4}$, should be permanently colored pink.) 
(e.g. 20 cc.), shake until the pink color disappears, and then finish the titration by the further addition of permanganate in portions of I cc. until the pink color persists after protracted shaking, say after $23.0 \mathrm{cc}$. in all have been added; to the third solution add at once $r .0 \mathrm{cc}$. less permanganate than the total volume used in the second case (e.g. $22.0 \mathrm{cc}$.), and continue the titration with the addition of $0.20 \mathrm{cc}$. portions, until the hot solution matches in color a solution prepared by the addition of o.ro cc. of the permanganate to $300 \mathrm{cc}$. of water. With the fourth solution, repeat this titration. If, for example, $22.60 \mathrm{cc}$. of the permanganate have been used in each of the last two titrations, then this quantity minus the o.Io cc. of the solution used for comparison should be taken as the volume actually required. Report the percentage of manganese in the ore.

NoTeS. - I. In case the treatment with hydrochloric acid and potassium chlorate should be insufficient to thoroughly decompose the ore (indicated by the presence of a dark-colored residue), the residue should be filtered off, washed, dried, and ignited in a platinum crucible. It should then be fused with sodium carbonate, the melt dissolved in hydrochloric acid, and the solution evaporated to dryness in a porcelain dish, to dehy. drate the silica. The final residue should be moistened with hydrochloric acid, taken up in water, and filtered into the Erlenmeyer flask containing the acid filtrate from the original residue. The resulting solution, which contains all the manganese, is then evaporated to a small volume, transferred to the measuring flask, and treated as described in the procedure.

2. Upon the addition of zinc oxide to the acid solution of the ore, the zinc oxide first neutralizes the acid with the formation of zinc chloride, and then precipitates the iron with the further formation of zinc chloride, according to the reaction:

$$
{ }_{2} \mathrm{FeCl}_{3}+{ }_{3} \mathrm{ZnO}+{ }_{3} \mathrm{H}_{2} \mathrm{O}=2 \mathrm{Fe}(\mathrm{OH})_{3}+{ }_{3} \mathrm{ZnCl}_{2} \text {. }
$$

In this way sufficient zinc-ion is introduced into the solution to insure the conversion of the manganese into the hydrated manganite of zinc.

3. Although it is often recommended to convert the chlorides in the solution into sulphates before the addition of zinc oxide, this treatment is not necessary. The titration of manganese in a dilute neutral solution with potassium permanganate is a very different thing from that of ferrous 
iron in a dilute acid solution containing chlorides. In the latter case, the ferrous iron catalyzes the reaction,

$$
2 \mathrm{KMnO}_{4}+16 \mathrm{HCl}=2 \mathrm{KCl}+2 \mathrm{MnCl}_{2}+5 \mathrm{Cl}_{2}+8 \mathrm{H}_{2} \mathrm{O} \text {, }
$$

some of the chlorine escapes, and there is a tendency to high results. In the former case, however, nothing is present in the solution to catalyze the reaction between the permanganate and the small quantity of hydrochloric acid which is formed; and, although the solution is hot, its acid concentration is so low that there is no danger from this source. Starting with $0.2000 \mathrm{~g}$. of an ore containing $20 \%$ of manganese, for example, the total quantity of acid formed in the titration (e.g. $4 \mathrm{KMnO}_{4}+5 \mathrm{ZnCl}_{2}$ $\left.+6 \mathrm{MnCl}_{2}+\mathrm{I}_{4} \mathrm{H}_{2} \mathrm{O}=4 \mathrm{KCl}+\mathrm{I} 8 \mathrm{HCl}+5 \mathrm{ZnO} . \mathrm{Mn}_{2} \mathrm{O}_{3}(\mathrm{OH})_{2}\right)$ weighs about $\frac{3 \mathrm{HCl}}{\mathrm{Mn}} \times 0.04$, or somewhat less than $0.1 \mathrm{~g}$; and this quantity in a volume of over $300 \mathrm{cc}$. would give an acid strength of less than 0.0 -or-normal.

4. The titration should be performed at $80-85^{\circ}$, and especial care should be taken not to heat the solution too hot.

5. For the greatest accuracy, in spite of all that has been said above, the permanganate solution should be standardized against a known quantity of manganese, weighed as $\mathrm{MnSO}_{4}$, under conditions similar to those to be used in the analysis. 


\section{IODOMETRIC PROCESSES}

Fundamental Considerations. Analyses which are based upon the volumetric determination of specific quantities of iodine are classed under the head of iodometric processes. In these processes, either a standard solution of iodine is used to effect a definite chemical action, or the iodine liberated in a reaction is volumetrically determined by titration with a suitable solution.

The estimation of iodine with standard thiosulphate solution, with the use of starch, is a very exact volumetric process; the titration may be performed in neutral or slightly acid solution. Moreover, if the iodine has previously been liberated from an excess of hydriodic acid by means of a specific oxidizing agent, then the results of the titration may be expressed directly in terms of this oxidizing agent itself. For example, the chromium in a sample of ore may be converted into the hexivalent condition, and subsequently determined on the basis of the reactions:

$$
\mathrm{K}_{2} \mathrm{Cr}_{2} \mathrm{O}_{7}+6 \mathrm{KI}+\mathrm{I}_{4} \mathrm{HCl}=8 \mathrm{KCl}+2 \mathrm{CrCl}_{3}+7 \mathrm{H}_{2} \mathrm{O}+3 \mathrm{I}_{2} \text {; }
$$

and

$$
\mathrm{I}_{2}+2 \mathrm{Na}_{2} \mathrm{~S}_{2} \mathrm{O}_{3}=2 \mathrm{NaI}+\mathrm{Na}_{2} \mathrm{~S}_{4} \mathrm{O}_{6} \text {. }
$$

Or, more simply expressed,

$$
\mathrm{Cr}_{2} \mathrm{O}_{7}-+6 \mathrm{I}^{-}+\mathrm{I}_{4} \mathrm{H}^{+}={ }_{2} \mathrm{Cr}^{+++}+7 \mathrm{H}_{2} \mathrm{O}+3 \mathrm{I}_{2} \text {; }
$$

and

$$
\mathrm{I}_{2}+2 \mathrm{~S}_{2} \mathrm{O}_{3}-=2 \mathrm{I}^{-}+\mathrm{S}_{4} \mathrm{O}_{6}-\text {. }
$$

As an oxidizing agent, iodine may act directly, as in the reaction with sodium thiosulphate, or it may act indirectly through hypoiodous acid, which it gives with water, as in the equations:

$$
\mathrm{I}_{2}+\mathrm{HOH} \leftrightarrow \mathrm{HI}+\mathrm{HOI} \text {; }
$$

and

$$
\mathrm{Na}_{2} \mathrm{HAsO}_{3}+\mathrm{HOI} \stackrel{\mathrm{HI}}{\rightarrow} \mathrm{Na}_{2} \mathrm{HAsO}_{4} \text {. }
$$

It will be seen from these equations that a one-tenth normal solution of iodine contains one tenth of a gram-atom, or 12.692 g. of available iodine per liter.

It is of interest to mention that while chlorine and bromine 
oxidize sodium thiosulphate partially to sulphate, iodine, under analytical conditions, oxidizes it wholly to tetrathionate.

The solubility of iodine in water is too small for the preparation of even a one tenth normal solution. In the.presence of sufficient potassium iodide, however, the iodine dissolves much more readily, owing to the formation of an unstable but soluble polyiodide of the formula $\mathrm{KI}_{3}$ :

$$
\mathrm{KI}+\mathrm{I}_{2} \leftrightarrows \mathrm{KI} \text {. } \mathrm{I}_{2} \text {, or } \mathrm{I}^{-}+\mathrm{I}_{2} \leftrightarrows\left(\mathrm{I} . \mathrm{I}_{2}\right)-
$$

In the presence of reducing agents iodine is removed from this equilibrium mixture, the reaction runs to completion from right to left, and the solution can be used as though it were a simple solution of iodine. The potassium iodide used in the preparation of the solution should weigh about $\mathbf{I} .5$ times as much as the iodine. Moreover, the presence of potassium iodide in the solution renders it possible to employ commercial iodine (which is apt to contain chlorine as an impurity) in the preparation of the standard solutions; the chlorine is removed according to the equation, $\mathrm{ICl}+\mathrm{KI}=\mathrm{KCl}+\mathrm{I}_{2}$.

In performing iodometric titrations in the presence of sulphuric acid, particular attention should be given to the maintenance of suitable analytical conditions. If, for example, it is desired to determine copper by titrating the iodine liberated in the reaction,

\section{$2 \mathrm{CuSO}_{4}+2 \mathrm{H}_{2} \mathrm{SO}_{4}+4 \mathrm{KI} \leftrightarrow \mathrm{Cu}_{2} \mathrm{I}_{2}+4 \mathrm{KHSO}_{4}+\mathrm{I}_{2}$,}

it is not sufficient to simply add potassium iodide and sulphuric acid in (unknown) excess. It must. be remembered that such a mixture will contain both sulphuric and hydriodic acid, and that if the concentration of either is too great, or if the solution is allowed to become at all warm, the determination is very apt to be spoiled :

$$
\begin{aligned}
& \mathrm{H}_{2} \mathrm{SO}_{4}+2 \mathrm{HI}=\mathrm{H}_{2} \mathrm{SO}_{3}+\mathrm{H}_{2} \mathrm{O}+\mathrm{I}_{2} ; \\
& \mathrm{H}_{2} \mathrm{SO}_{4}+6 \mathrm{HI}=\mathrm{S}+4 \mathrm{H}_{2} \mathrm{O}+3 \mathrm{I}_{2} ;
\end{aligned}
$$

or, in extreme cases,

$$
\mathrm{H}_{2} \mathrm{SO}_{4}+8 \mathrm{HI}=\mathrm{H}_{2} \mathrm{~S}+{ }_{4} \mathrm{H}_{2} \mathrm{O}+{ }_{4} \mathrm{I}_{2} \text {. }
$$


Other things being equal, when acid solutions are required, it is better to use acetic acid or dilute hydrochloric acid.

In direct titrations with iodine, e.g. in the presence of sodium bicarbonate, it is best to work in the absence of ammonium salts. Such solutions are very faintly alkaline, especially if at all warm; and in the presence of ammonium salts ammonia is apt to be liberated, which is not entirely without influence upon the titration.

Iodine solutions should not be placed in burettes with rubber stopcocks.

Determination of the End-point. A single drop of one-tenth normal iodine solution imparts a distinct tint to $200 \mathrm{cc}$. of water, and in many titrations with this solution no other indicator is required. If, however, the solution to be titrated contains colored substances, or if the greatest possible accuracy is demanded, a solution of starch should be used as an indicator. Under the proper conditions, the presence of one part of free iodine in several millions of solution can be recognized with this indicator, but the sensitiveness of the reaction and the color produced are affected by a number of factors. The test is decidedly more sensitive when the concentration of iodide-ion (and of hydrogen-ion ${ }^{1}$ ) is not too low, and when the quantity of starch present is sufficient to give a deep blue color.

Under less favorable conditions, the starch may give a greenish or a reddish color; or it may be very unreliable, as in solutions containing an abnormally low iodide-ion concentration. However, since the standard iodine solution always contains potassium iodide, and since an iodide is always one product of the titration, there is ordinarily not much danger from this source. Attention should be directed mainly toward the observance of uniform conditions in all related titrations: the volumes of the

${ }^{1}$ Like iodine, the blue iodo-starch is incapable of existence in the presence of hydroxide-ion in appreciable quantity, and titrations therefore should not be attempted in the presence of alkali hydroxides or carbonates. In cold, dilute solution, however, alkali bicarbonates have no bad influence. 
solutions titrated should be approximately equal, the starch solution should be properly prepared, and the same quantity of it should be added for each titration. Finally, all titrations should be made in the cold; the iodo-starch blue is discharged by heat.

Preparation of the Starch Solution. Rub I g. of powdered starch with a little cold water to a thin paste, and slowly add this to $200 \mathrm{cc}$. of boiling water, stirring the hot mixture until an almost clear solution is obtained. Allow this to settle, and filter off the liquid. Use $5 \mathrm{cc}$. of this liquid for each titration.

A " soluble starch" which is in the market is more convenient, since with it filtration is unnecessary. A solution made by adding $200 \mathrm{cc}$. of boiling water to I $\mathrm{g}$. of this starch, previously mixed with a little cold water, serves the purpose well. Use 5 cc. of this solution for each titration.

In either case, the starch solution should be freshly prepared. If a great many titrations are to be made, however, it is advisable to prepare a liter of the starch solution; a number of small (50-100 cc.) bottles, filled with this solution, should be heated for at least an hour in boiling water, and, while still in the bath, they should be sealed with paraffined stoppers of soft cork. Thus sterilized and preserved, the solution will remain sensitive for many months. After a bottle has been opened, mold is likely to form within a few days.

\section{THE PREPARATION AND STANDARDIZATION OF APPROX- IMATELY ONE TENTH NORMAL SOLUTIONS OF IODINE AND SODIUM THIOSULPHATE}

Procedure. Weigh out on the rough balance $6.3-6.4 \mathrm{~g}$. of commercial iodine, add it to a solution of $9 \mathrm{~g}$. of potassium iodide in $25 \mathrm{cc}$. of water, in an Erlenmeyer flask, and agitate the mixture until the iodine is completely dissolved. Dilute the solution to $500 \mathrm{cc}$, in a measuring flask, and mix it thoroughly. 
Heat $600-700 \mathrm{cc}$. of distilled water in a large flask and boil for about 5 minutes. Stopper the flask loosely, and allow the water to cool. Weigh out $\mathrm{I} 2.5 \mathrm{~g}$. of sodium thiosulphate, $\mathrm{Na}_{2} \mathrm{~S}_{2} \mathrm{O}_{3} \cdot{ }_{5} \mathrm{H}_{2} \mathrm{O}$, introduce it into a 500-cc. measuring flask, and dissolve it in about $200 \mathrm{cc}$. of the cold, freshly boiled water. Finally dilute to the mark with more of the same water, and mix thoroughly.

After these solutions have come to the room temperature, fill a burette with each (see p. I 50 ), and measure out $20 \mathrm{cc}$. of the thiosulphate solution, into an Erlenmeyer flask. Add to this I $25 \mathrm{cc}$. of water and $5 \mathrm{cc}$. of starch solution, and then, with shaking, run in the iodine solution until the faintest blue tinge persists in the mixture. If the end-point is overstepped, titrate back with the thiosulphate solution. (All waste solutions containing iodine and potassium iodide should be poured into the vessel provided for iodine residues.) From the (corrected) data, calculate the ratio of the two solutions, repeating the titration until satisfactory checks are obtained.

Weigh out into $500 \mathrm{cc}$. Erlenmeyer flasks two 0.12-0.13 g. portions of arsenious oxide, and in each case warm gently with ro cc. of 6-normal sodium hydroxide. To the resulting solution add $50 \mathrm{cc}$. of water and 2 drops of methyl orange, and then cautiously add 6-normal hydrochloric acid, 2 or 3 drops in excess. Finally add $-75 \mathrm{cc}$. of a cold solution of sodium bicarbonate (made by dissolving Io $\mathrm{g}$. of the pure salt in $\mathrm{r} 5 \mathrm{O} \mathrm{cc}$. of water) and $5 \mathrm{cc}$. of starch solution, and then titrate with the iodine. Do not overstep the end-point. From the data obtained, calculate the normality factor of the iodine. (Duplicate values should agree within two parts in one thousand.) Also calculate the normality factor of the thiosulphate solution.

NoTES. - I. Iodine solutions, like chlorine water, are acted upon by sunlight; and, also, iodine is readily volatile. Iodine solutions, therefore, should be kept in a cool, dark place, and should be restandardized at suitable intervals. 
2. Standard thiosulphate solutions may also be prepared by dissolving the theoretical quantity of the purified salt in cold water (free from carbonic acid) and diluting to the required volume. Such solutions are quite stable, and, if protected from carbon dioxide, in a cool, dark place, they may be kept for months without appreciable change.

3. Carbonic acid causes a slow decomposition of the thiosulphate solution, with the formation of free sulphur and sulphurous acid; and, since sulphurous acid acts in the same way, the decomposition once started becomes progressive:

and

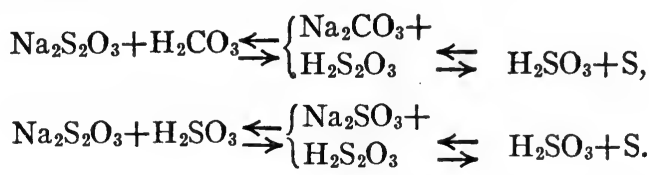

The reducing value of the solution increases gradually as the decomposition progresses; i.e. the solution apparently becomes stronger. When it is considered that in this decomposition each molecule of thiosulphate yields one molecule of sulphite, the greater reducing value is readily understood; for

while

$$
\begin{aligned}
2 \mathrm{Na}_{2} \mathrm{~S}_{2} \mathrm{O}_{3}+\mathrm{I}_{2} & =\mathrm{Na}_{2} \mathrm{~S}_{4} \mathrm{O}_{6}+{ }_{2} \mathrm{NaI}, \\
I \mathrm{Na}_{2} \mathrm{SO}_{3}+\mathrm{I}_{2}+\mathrm{H}_{2} \mathrm{O} & =\mathrm{Na}_{2} \mathrm{SO}_{4}+{ }_{2} \mathrm{HI} .
\end{aligned}
$$

4. Although the standardization of thiosulphate solution against a weighed quantity of pure iodine is sometimes advocated, this procedure is by far too troublesome; it is much better to use a carefully standardized iodine solution. Instead of this, however, a definite weight of potassium bromate, iodate, or dichromate, all of which are readily obtainable in a pure condition, may be added to an excess of pure potassium iodide in slightly acid solution, with the liberation of a definite quantity of iodine; and this may be used in the standardization. In the same way, the thiosulphate solution may also be standardized indirectly against a standard solution of potassium permanganate.

5. Arsenious oxide dissolves most readily in caustic alkalies, and for this reason the sodium hydroxide is used. The sodium hydroxide, however, must subsequently be removed, since otherwise it would react with the iodine; hence the acidification with hydrochloric acid. The purpose of the bicarbonate, which under the analytical conditions is without action upon the.iodine, is to destroy the hydriodic acid formed in the reversible action, $\mathrm{As}_{2} \mathrm{O}_{3}+{ }_{2} \mathrm{I}_{2}+{ }_{2} \mathrm{H}_{2} \mathrm{O} \leftrightarrows \mathrm{As}_{2} \mathrm{O}_{5}+4 \mathrm{HI}$, and thus drive the action to completion to the right. The reaction may then be written:

$\mathrm{Na}_{2} \mathrm{HAsO}_{3}+\mathrm{I}_{2}+2 \mathrm{NaHCO}_{3}=\mathrm{Na}_{2} \mathrm{HAsO}_{4}+2 \cdot \mathrm{NaI}+2 \mathrm{CO}_{2}+\mathrm{H}_{2} \mathrm{O}$. 
6. Since the addition of iodine in excess to the weakly basic bicarbonate solution is likely to lead to a slight degree of action, it is best in this titration not to overstep the end-point.

7. Iodine is a rather expensive chemical and it is well worth while to recover it from the united residues of a large class. 


\section{THE DETERMINATION OF ANTIMONY IN STIBNITE}

The stibnite should be an ore practically free from arsenic and iron, and, with the exception of a siliceous residue, it should be wholly soluble in hydrochloric acid.

Procedure. Weigh out into $150 \mathrm{cc}$. beakers $0.20 \mathrm{~g}$. portions of the finely pulverized ore, add to each about $0.3 \mathrm{~g}$. of solid potassium chloride and $5 \mathrm{cc}$. of I2-normal hydrochloric acid (effervescence), and allow to stand for ro minutes; then warm on the steam bath until the residue is white. Add $2.5 \mathrm{~g}$. of pulverized tartaric acid, and continue the heating for 15 minutes; with the addition of a little of the hydrochloric acid, if necessary, to prevent the exposure of the bottom of the beaker.

Now add water to the mixture, a little at a time, with care to stop if there is any indication of the precipitation of antimony sulphide; in such a case, warm the mixture until the orangered substance disappears, and again proceed with the gradual addition of water, until the clear liquid has a volume of roo cc. Neutralize the cold solution with sodium hydroxide (methyl orange), and then add 6-normal hydrochloric acid, 2 drops in excess.

Transfer the solution quantitatively to a $500 \mathrm{cc}$. Erlenmeyer flask, washing out the beaker with three 25 cc. portions of sodium bicarbonate solution (made by dissolving $20 \mathrm{~g}$. of the pure salt in $300 \mathrm{cc}$. of cold water), add $5 \mathrm{cc}$. of starch solution, and titrate with the standard iodine solution, with care not to overstep the end-point. Report the percentage of antimony found.

Notes. - r. Stibnite is an important material in the manufacture of primers for cartridges. The pure substance has the composition represented by $\mathrm{Sb}_{2} \mathrm{~S}_{3}$, but the commercial mineral may also contain the compounds $\mathrm{Sb}_{2} \mathrm{OS}_{2}$ and $\mathrm{Sb}_{2} \mathrm{O}_{4}$, besides iron, arsenic, lead, siliceous gangue, etc. Upon warming the ore with hydrochloric acid, the antimony is extracted, and hydrogen sulphide evolved: $\mathrm{Sb}_{2} \mathrm{~S}_{3}+6 \mathrm{HCl}=2 \mathrm{SbCl}_{3}+{ }_{3} \mathrm{H}_{2} \mathrm{~S}$. The student will recall, however, that this reaction is reversible; if the antimony is to 
be kept in solution, the expulsion of hydrogen sulphide must be complete before the final dilution.

2. Antimony trichloride, in the presence of strong hydrochloric acid, is slightly volatile; with potassium chloride, however, it forms a less volatile double salt. With careful heating, especially in the presence of potassium chloride, no error need be feared from this source.

3. Antimony trichloride and water interact to give insoluble antimonyl chloride, $\mathrm{SbOCl}$, but this with tartaric acid forms a soluble antimonyl compound similar to tartar emetic:

$$
\mathrm{SbOCl}+\mathrm{H}_{2} \mathrm{C}_{4} \mathrm{H}_{4} \mathrm{O}_{6}=\mathrm{H}(\mathrm{SbO}) \mathrm{C}_{4} \mathrm{H}_{4} \mathrm{O}_{6}+\mathrm{HCl} \text {. }
$$

The tartaric acid, therefore, is added to prevent the precipitation of basic compounds of antimony in the subsequent treatment of the solution. If a white precipitate should be obtained, however, the solution should be rejected.

4. The reaction between the iodine and the antimonyl tartrate is not so simple, but for purposes of calculation it is accurately expressed by the equation, $\mathrm{Sb}_{2} \mathrm{O}_{3}+2 \mathrm{I}_{2}+2 \mathrm{H}_{2} \mathrm{O} \leftrightarrows \mathrm{Sb}_{2} \mathrm{O}_{5}+4 \mathrm{HI}$. The purpose of the bicarbonate is here also to neutralize the hydriodic acid formed, and thereby drive the oxidation to completion. The sodium hydroxide previously added neutralizes most of the acid and makes it easy to provide for the presence of a known quantity of sodium bicarbonate in the titration; the solution should be distinctly acid when the bicarbonate is added.

5. If the ore to be analyzed contains more than traces of iron, it is dissolved in hydrochloric acid, the antimony precipitated with hydrogen sulphide, and the washed precipitate redissolved in hydrochloric acid and determined as above. In case arsenic also is present, a somewhat more complicated treatment is necessary. 


\section{THE DETERMINATION OF CHROMIUM IN CHROMITE}

Procedure. Weigh out into bright iron crucibles two portions of the finely pulverized ore, each sufficient to contain about $45 \mathrm{mg}$. of chromium. Also weigh out roughly upon watch glasses two $4 \mathrm{~g}$. portions of pure sodium peroxide. (Owing to the tendency of the peroxide to absorb moisture, the first portion should be mixed with one sample before the second portion is removed from the container.) Transfer about $3 \mathrm{~g}$. of the peroxide to each crucible, and mix this with the ore by means of a dry rod; remove any adhering particles from the rod by stirring with it the remaining peroxide, and transfer the latter to the surface of the mixture. Gradually heat the crucible upon a triangle, with a small movable flame, until the mixture melts; and continue to heat at about this temperature for 5 or 6 minutes. Then allow the melt to cool.

In a tall $250 \mathrm{cc}$. beaker, treat the crucible and its contents with cold water, with great care to avoid loss by effervescence. As soon as the action slows down, heat the mixture gradually, finally at the boiling temperature, until there is no further evolution of oxygen. Take out and wash off the crucible, add sufficient 6-normal hydrochloric acid (calculated) to almost neutralize the liquid, and filter; receive the filtrate in a large Erlenmeyer flask. To the filtrate and washings, which should be slightly alkaline, add about $0.2 \mathrm{~g}$. of sodium peroxide, boil for several minutes, and acidify with 6-normal hydrochloric acid, adding $5 \mathrm{cc}$. in excess. The volume at this point should be about $200 \mathrm{cc}$. Mix with this solution $\mathrm{I} .5 \mathrm{~g}$. of pure potassium iodide, and titrate at once with the standard thiosulphate solution until the brown (iodine) color becomes faint; then add 5. cc. of starch solution, and continue the titration cautiously until the solution becomes pale green $\left(\mathrm{CrCl}_{3}\right)$ with no tinge of blue. The end-point, though very sharp, may easily be passed through carelessness. It is well to have a white surface under the flask.

Report the percentage of chromium in the ore. 
Notes. - I. Fused sodium peroxide attacks most materials; although it attacks iron and nickel, crucibles of these metals may nevertheless be used in this case, but great care should be taken not to raise the temperature too high.

2. Chromite is an ore consisting largely of ferrous chromite, $\mathrm{Fe}\left(\mathrm{CrO}_{2}\right)_{2}$. Fused sodium peroxide oxidizes the ferrous iron to sodium ferrate and the chromium to sodium chromate:

$$
2\left(\mathrm{FeO} . \mathrm{Cr}_{2} \mathrm{O}_{3}\right)+\text { ro } \mathrm{Na}_{2} \mathrm{O}_{2}=4 \mathrm{Na}_{2} \mathrm{CrO}_{4}+2 \mathrm{Na}_{2} \mathrm{FeO}_{4}+4 \mathrm{Na}_{2} \mathrm{O} \text {. }
$$

Upon the addition of water to the melt, the chromate dissolves, the ferrate is decomposed into sodium hydroxide, ferric oxide, and oxygen, and the excess of the peroxide yields sodium hydroxide and oxygen; the liquid is boiled in order to complete the destruction of the peroxide, since if present it would lead to the formation of perchromate upon acidification.

The alkaline chromate solution is always subject to a slight partial reduction when filtered through paper; hence the addition to the filtrate of a little sodium peroxide, followed by renewed boiling.

3. The mixture should not be acidified before filtration, because in that case iron would enter the solution and cause large errors in the titration. The partial neutralization is to prevent the destruction of the filter paper by the alkaline liquid.

4. Upon the addition of potassium iodide in excess to the acidified fusion extract, iodine is liberated quantitatively according to the equation,

$$
\mathrm{Cr}_{2} \mathrm{O}_{7}--+6 \mathrm{I}^{-}+\mathrm{I}_{4} \mathrm{H}^{+}={ }_{2} \mathrm{Cr}^{+++}+7 \mathrm{H}_{2} \mathrm{O}+{ }_{3} \mathrm{I}_{2} \text {. }
$$

5. Instead of employing this method, it is of course possible to prepare the acid fusion extract as described in the procedure, and to add to it (in excess) a known weight of pure ferrous ammonium sulphate; the excess of this substance can then be titrated with standard dichromate solution. In that case, a larger sample of the ore may be taken for analysis. 


\section{THE DETERMINATION OF LEAD IN AN ORE}

Procedure. Weigh out two samples of the finely ground ore sufficient to contain about $0.20 \mathrm{~g}$. of lead $(0.28-0.29 \mathrm{~g}$. of a $70 \%$ ore), and treat each as follows: Moisten the sample with water, add I5 cc. of I2-normal hydrochloric acid, and evaporate on the steam bath to about $5 \mathrm{cc}$. Add $3 \mathrm{cc}$. of strong nitric acid, evaporate nearly to dryness, then add $20 \mathrm{cc}$. of 6 -normal hydrochloric acid and again heat to bring all the lead chloride into solution. Add $20 \mathrm{cc}$. of 6-normal sulphuric acid and evaporate to white fumes. Allow to cool, add $50 \mathrm{cc}$. of water, boil, and then add I 5 cc. of alcohol; stir, allow to settle, and filter. Wash the lead sulphate and gangue six times with ro-cc. portions of 0.5-normal sulphuric acid ( $15 \mathrm{cc}$. of 6-normal acid in $165 \mathrm{cc}$. of water), transfer the residue to a small beaker by means of a jet of water, and heat it gently for a few minutes with $20 \mathrm{cc}$. of ammonium acetate solution $;^{1}$ filter the liquid through the original filter and wash the latter with small portions of the hot ammonium acetate solution. Dilute the extract to $\mathrm{r}_{50} \mathrm{cc}$, heat to boiling and add from a pipette ro cc. of potassium dichromate solution. ${ }^{2}$ Boil the mixture gently for ro minutes, filter off the precipitate of lead chromate and wash the filter and precipitate about ten times with Io cc. portions of dilute ammonium acetate solution $(25 \mathrm{cc}$. of the extraction solution diluted to $250 \mathrm{cc}$.), until the excess of potassium chromate is completely removed.

Now place a clean 500-cc. Erlenmeyer flask under the funnel, and with a jet of cold, acid, sodium chloride solution ${ }^{3}$ stir up and dissolve the precipitate; continue washing with the same liquid until every trace of color is removed from the filter. In any case, use at least $50 \mathrm{cc}$. of the liquid. Finally dilute to

1 Made by neutralizing $30 \%$ acetic acid with 6-normal ammonia, and then adding a slight excess of ammonia.

${ }^{2}$ A solution containing 75 g. of $\mathrm{K}_{2} \mathrm{Cr}_{2} \mathrm{O}_{7}$ per liter.

3 Mix ro cc. of 12 -normal hydrochloric acid with ${ }_{5} 5 \mathrm{cc}$. of water, and add this mixture to $100 \mathrm{cc}$. of a saturated solution of sodium chloride. 
I 50 cc., add I g. of potassium iodide, mix, and titrate at once with a solution of sodium thiosulphate (which has been standardized in the same way against test lead, see Note 5) until the brown color becomes faint; then add $5 \mathrm{cc}$. of starch solution, and continue the titration cautiously until the solution becomes pale green $\left(\mathrm{CrCl}_{3}\right)$ with no tinge of blue. The end-point is very sharp, but without great care it may easily be passed. It is best to have a white surface under the flask.

Report the percentage of lead in the ore.

Notes. - I. The ore is first heated with strong hydrochloric acid in order to expel most of the sulphur. Nitro-hydrochloric acid is then used to decompose any refractory sulphides. Upon evaporating the chloride solution to white fumes with sulphuric acid, the volatile acids in which lead sulphate is slightly soluble are completely expelled, and upon dilution with water, especially if alcohol is added, the lead is all left in the residue as lead sulphate.

2. Lead sulphate is readily dissolved by ammonium acetate solution, owing to the exceptional behavior of lead acetate with respect to ionization (see Part I), leaving the siliceous gangue, $\mathrm{BaSO}_{4}$, etc., as a residue.

3. While lead is not precipitated from solutions containing a large excess of acetate ion by sulphates, the addition of a soluble chromate causes the precipitation of lead chromate. This behavior is due to the fact that such solutions contain $\mathrm{Pb}^{++}$-ion at an extremely low concentration (owing to the presence of the lead mainly in the form of intermediate or complex ions, as $\left(\mathrm{Pb} . \mathrm{C}_{2} \mathrm{H}_{3} \mathrm{O}_{2}\right)^{+},\left[\mathrm{Pb}\left(\mathrm{C}_{2} \mathrm{H}_{3} \mathrm{O}_{2}\right)_{3}\right]^{-}$, etc.), and also to the fact that lead sulphate is very much more soluble than lead chromate; the lead-ion concentration is still great enough in such solutions to cause the solubility product of lead chromate to be exceeded upon the addition of potassium chromate in excess.

4. The solubility of lead chromate in the acid chloride solution is due on the one hand to the lowered concentration of the chromate ion, owing to the formation of non-ionized $\mathrm{H}_{2} \mathrm{CrO}_{4}, \mathrm{HCrO}_{4}^{-}$, etc., and on the other hand to the great tendency of lead ion to form soluble complexes with chloride solutions. (Cf. the solubility of silver chloride in chloride solutions.)

5. The reaction of the acid solution with potassium iodide is most simply represented by the equation,

$$
\mathrm{Cr}_{2} \mathrm{O}_{7}-+6 \mathrm{I}^{-}+\mathrm{I}_{4} \mathrm{H}^{+}=2 \mathrm{Cr}^{+++}+7 \mathrm{H}_{2} \mathrm{O}+3 \mathrm{I}_{2} ;
$$


from this it may be seen that one atom of lead (as $\mathrm{PbCrO}_{4}$ ) leads to the liberation of 3 atoms of iodine. But, since the composition of the lead chromate varies slightly with the conditions, the thiosulphate solution must be standardized under identical conditions against a known amount of lead. In this case, $0.20 \mathrm{~g}$. of test lead should be dissolved in $5 \mathrm{cc}$. of 6-normal nitric acid, the solution evaporated to white fumes with $20 \mathrm{cc}$. of 6-normal sulphuric acid, and the subsequent operations carried out as described in the procedure. 


\section{THE DETERMINATION OF COPPER IN AN ORE}

Principle. This method is based upon the reaction which takes place upon the addition of potassium iodide to a slightly acid copper salt solution; cuprous iodide is precipitated as a cream-colored powder, and iodine is set free:

$$
{ }_{2} \mathrm{CuSO}_{4}+4 \mathrm{KI}=2 \mathrm{~K}_{2} \mathrm{SO}_{4}+\mathrm{Cu}_{2} \mathrm{I}_{2}+\mathrm{I}_{2} \text {. }
$$

The iodine is promptly titrated with a standard thiosulphate solution.

Standardization of the Thiosulphate Solution. Weigh accurately two portions of pure bright copper wire or foil, of 0.150.16 g. each, and, in $250 \mathrm{cc}$. Erlenmeyer flasks, dissolve these in 5 cc. portions of 6-normal nitric acid. Dilute each solution to I 5 cc. and boil to expel the red fumes; then dilute to $25 \mathrm{cc}$. and add ammonia (sp. gr., 0.90) in slight excess. Again boil until the ammonia odor is faint, add $80 \%$ acetic acid, $2-3 \mathrm{cc}$. in excess, and boil for a moment longer, agitating the flask in a holder to prevent bumping. Cool to room temperature, dilute to $40 \mathrm{cc}$, add a solution of $3 \mathrm{~g}$. of potassium iodide in Io cc. of water, and titrate at once with the approximately tenth-normal thiosulphate solution to a faint brown tinge; add $5 \mathrm{cc}$. of starch solution, and continue the titration until the last faint lilac tint is removed by a single drop. Do not overstep the end-point. From the data obtained, calculate the value of the solution per cubic centimeter in terms of copper.

Analytical Procedure. Weigh out into $300 \mathrm{cc}$. beakers samples of the ore sufficient to furnish about 0.I $5 \mathrm{~g}$. of copper, and treat each as follows: Add ro cc. of hydrochloric acid (sp. gr., I.r9) and 5 cc. of nitric acid (sp. gr., I.42) and heat in the covered beaker on the hot plate until decomposition is complete, adding more of the acids if necessary, and enough water at the end to hold all soluble salts in solution. Then add $\mathrm{I}_{5} \mathrm{cc}$. of 6-normal sulphuric acid, and continue the heating until abundant white fumes begin to come off. . Cool, add 50-6o cc. of water, boil for 
a moment, and allow to stand, hot, until any anhydrous ferric sulphate has dissolved. Finally, filter off from any lead sulphate, gangue, and sulphur, receiving the filtrate and washings in a $300 \mathrm{cc}$. beaker. Now add a solution of $5 \mathrm{~g}$. of sodium thiosulphate in $25 \mathrm{cc}$. of water, boil to coagulate the precipitate, and filter, transferring the precipitate quantitatively to the filter by means of hot water. Dry the precipitate on the filter.

Place the precipitate, together with the filter, in a porcelain crucible, ignite gently until the filter is consumed, and allow to cool. Transfer the bulk of the precipitate to a $250 \mathrm{cc}$. Erlenmeyer flask, and set aside. To dissolve the last portions of the precipitate from the crucible, add $3 \mathrm{cc}$. of concentrated nitric acid and 2 cc. of water, and warm gently on the hot plate, finally pouring the acid solution into the flask containing the bulk of the precipitate, and washing out the crucible with a few small portions of 6-normal nitric acid. Heat the mixture in the flask until the decomposition is complete, dilute to $25 \mathrm{cc}$., boil, add ammonia in slight excess, and heat until the odor is faint. Add $80 \%$ acetic acid, $2-3 \mathrm{cc}$. in excess, and boil for a moment, vigorously agitating the flask to prevent bumping. Cool to room temperature, dilute to $40 \mathrm{cc}$., add $3 \mathrm{~g}$. of potassium iodide dissolved in ro cc. of water, and titrate at once with the thiosulphate solution, as previously described. Report the percentage of copper in the ore.

Notes. - r. Since iron and other elements likely to be present interfere with the process, the copper must be separated from these. Lead is first removed by means of sulphuric acid, after which the copper is precipitated from the hot, acid solution by means of sodium thiosulphate; this gives a flocculent precipitate of cuprous sulphide mixed with sulphur, which filters readily and can be washed with hot water without fear of oxidation. Arsenic and antimony, if present, are also precipitated, but under the treatment prescribed the usual quantities of these elements are without influence. They are mostly volatilized during the ignition. If antimony is present in appreciable quantity, it is perhaps better to filter the solution before the addition of the ammonia.

2. In order to obtain the best results it is necessary to standardize the 
thiosulphate solution against pure metallic copper. When this is done the method is very accurate; otherwise the results are not so good. For example, a thiosulphate solution which (titrated against a freshly standardized iodine solution) had a calculated copper value of $0.00608 \mathrm{~g}$. per cubic centimeter, was found upon standardization against pure copper to have a value of $0.006 \mathrm{II} \mathrm{g}$. per cubic centimeter.

3. Since nitrous fumes liberate iodine from potassium iodide, they must be completely expelled by boiling before the addition of the salt. The expulsion of the last traces of these fumes is insured by boiling the solution after it has been acidified with acetic acid.

4. The return of the blue tinge in the liquid after long standing is of no significance, but a quick return which is not prevented from recurring by the addition of a single drop of the thiosulphate solution is usually an evidence of faulty work.

5. In such a case, or if the end-point has accidentally been passed, the same sample may be prepared anew for titration: Add Io cc. of concentrated nitric acid, and heat very cautiously, with great care not to allow the mixture to foam over. After most of the iodine has been expelled, manipulate the flask (in a holder) over a free flame and boil the solution down rapidly to a volume of $5^{-10} \mathrm{cc}$. Dilute to $25 \mathrm{cc}$. with water, boil, add ammonia in slight excess, and finish as described in the procedure.

6. In the electrolytic determination of copper in ores containing arsenic and other interfering substances, a satisfactory copper solution is most readily prepared by dissolving the ignited thiosulphate precipitate in a suitable quantity of strong nitric acid, with subsequent dilution to the required volume. 


\section{PRECIPITATION METHODS}

General Discussion. Perhaps the simplest example of a precipitation process is that furnished by the method for silver which was originated by Gay-Lussac in 1832 , and which is still widely used in determining the fineness of silver bullion. This process is based upon the reaction between silver nitrate and a standard solution of sodium chloride, which runs to completion in consequence of the union of silver-ion with chloride-ion to furnish insoluble silver chloride.

When the silver chloride first separates it is finely divided, and a very minute quantity can easily be recognized. Upon vigorously shaking the solution, the precipitate coagulates and settles, leaving the supernatant liquid bright and clear. Hence, if silver nitrate is titrated with a solution of sodium chloride, with shaking in a glass-stoppered bottle after each addition, the point at which the further addition of the standard solution ceases to produce a precipitate can readily be determined. Near the end-point it is customary to use a standard solution of one tenth the value of that used at the start.

In the case of this reaction, this method of determining the end-point admits of a very high degree of accuracy, and it is the method in use at the government mints. Since, however, it is rather tedious and demands considerable skill and experience, a slightly less accurate but much more convenient method is generally employed.

Silver thiocyanate is perfectly white, and even less soluble than silver chloride; it is therefore possible to titrate silver very accurately with a standard solution of an alkali thiocyanate. If the solution contains ferric alum, and also nitric acid to prevent its hydrolysis, the addition of the slightest excess of the thiocyanate solution can be readily recognized by the pink tint which it imparts to the mixture. This method (Volhard's) is also suitable for the determination of the halogens (except fluorine) and of certain other ions which give silver compounds 
insoluble in dilute nitric acid. A measured volume of standard silver nitrate solution is added in excess, and the excess subsequently determined by means of the standard thiocyanate solution.

THE PREPARATION AND STANDARDIZATION OF APPROXIMATELY ONE TENTH NORMAL SOLUTIONS OF SILVER NITRATE AND AMMONIUM THIOCYANATE

Procedure. Dissolve $4.0 \mathrm{~g}$. of ammonium thiocyanate (or $5.0 \mathrm{~g}$. of the potassium salt) in water and dilute the solution to $500 \mathrm{cc}$. Also dissolve $8.5 \mathrm{~g}$. of silver nitrate in water and dilute the solution to $500 \mathrm{cc}$. Further, mix 10 cc. of 6-normal nitric acid with $40 \mathrm{cc}$. of water, heat the solution to boiling, and dissolve in the hot liquid $5 \mathrm{~g}$. of pure ferric alum. Allow the solution to cool, and keep it for use as an indicator.

Now fill the burettes with the respective solutions, placing the silver nitrate solution in a glass-stoppered burette. (Observe the usual precautions, and place all solutions and precipitates containing silver in the receptacle for silver residues.) Run out $20 \mathrm{cc}$. of the silver nitrate into an Erlenmeyer flask, dilute to $50 \mathrm{cc}$., add Io cc. of freshly boiled 6-normal nitric acid, and $5 \mathrm{cc}$. of the indicator. With vigorous shaking, run in the thiocyanate solution until a faint pink tinge is imparted to the mixture. If the end-point is overstepped, titrate back with the silver nitrate solution. Calculate the ratio of the thiocyanate to the silver nitrate solution, and repeat the operation until the values found agree within two parts in one thousand.

Finally, standardize the silver nitrate solution, as follows: Weigh out portions of pure sodium chloride, of 0.12-0.14 g. each, dissolve these in 75 -cc. portions of water, heat to boiling, and with stirring run into each from a burette $25.00 \mathrm{cc}$. of the silver nitrate solution. Add Io cc. of freshly boiled 6-normal nitric acid, stir, and filter, washing the precipitate by decantation with several small portions of hot distilled water, and pouring these slowly over the filter; the united filtrate and washings 
should have a volume of about $150 \mathrm{cc}$. To this solution add $5 \mathrm{cc}$. of the indicator, and titrate the excess of silver with the thiocyanate solution, as already described. From the data obtained, calculate the normality factor of the silver nitrate solution; and from the mean of the duplicate values, which should agree within two parts in a thousand, calculate the normality factor of the thiocyanate solution.

Notes. - I. The reactions between the thiocyanate and the indicator are essentially as follows:

$$
\mathrm{Fe}^{+++}+6 \mathrm{CNS}^{-} \longrightarrow \mathrm{Fe}(\mathrm{CNS})_{3}+{ }_{3} \mathrm{CNS}^{-} \leftrightarrows\left[\mathrm{Fe}(\mathrm{CNS})_{6}\right]-
$$

It will be recalled that in testing for ferric iron with potassium thiocyanate, it is necessary to add a large excess of the latter in order to detect the smallest possible quantity of iron. In the same way, when using ferric iron as an indicator for thiocyanate, it is necessary to provide a high concentration of the former in order to detect the slightest possible excess of the thiocyanate in the solution. The reactions which give rise to the colored substances are reversible, but in the presence of a large excess of one of the colorless constituents the dissociation of the colored substances is prevented by mass action.

2. Nitric acid is added to the solution to be titrated in order to prevent the hydrolysis of the ferric salt, which would impart a brownish red color to the mixture It is boiled to free it from nitrous fumes, though this is of less importande here than testing for iron in the presence of nitric acid; nitrous fumes color the thious nate pink.

3. Sodium chloride may easily be obtained pure by filtering a concentrated solution of the commercial salt, saturating it with hydrogen chloride gas, and filtering off the precipitate. The latter is washed with strong hydrochloric acid and dried at $150^{\circ}$, or higher. $\therefore$

4. Standard solutions of silver nitrate can of course be prepared by the solution of the calculated amount of pure metallic silver in nitric acid, and dilution to the required volume; or by means of the calculated weight of pure silver nitrate. 


\section{THE DETERMINATION OF CHLORINE IN A SOLUBLE CHLORIDE}

The sample may be an artificial mixture of the chloride and carbonate of sodium.

Procedure. Weigh out into 300-cc. beakers, two portions, each sufficient to contain about $0.12 \mathrm{~g}$. of sodium chloride, and treat each as follows: Dissolve the sample in $50 \mathrm{cc}$. of water, run in from a burette $30.00 \mathrm{cc}$. of the standard silver nitrate solution, and carefully acidify the mixture with dilute nitric acid. Heat to boiling, see that the liquid is distinctly acid, and filter. Receive the filtrate and washings in a 300-cc. Erlenmeyer flask. To the united filtrate and washings, which should have a volume of about 150 cc., add ro cc. of 6-normal nitric acid and 5 cc. of the indicator solution, and titrate with the standard thiocyanate solution, as already described. Calculate the percentage of chlorine in the sample.

Notes. - I. Since silver chloride is several times as soluble as silver thiocyanate, the former must be filtered off before the titration of the excess of silver nitrate; otherwise the silver chloride would react with the thiocyanate solution and render the end-point uncertain. This behavior is best represented by the following system of equilibria:

$$
\begin{aligned}
& \underset{(\text { Solid })(\text { Diss'd })}{\mathrm{AgCl}} \stackrel{\mathrm{AgCl}}{\longleftrightarrow}\left\{\begin{array}{l}
\mathrm{Cl}^{-}+ \\
\mathrm{Ag}^{+} \quad\left(+\mathrm{CNS}^{-} \leftrightarrows \mathrm{AgCNS}^{\leftrightarrows} \mathrm{AgCNS}\right) .
\end{array}\right. \\
& \text { (Diss'd) }
\end{aligned}
$$

That is, if the silver chloride were left in the mixture during the titration, owing to the slow conversion of the soluble (colored) thiocyanate compounds into insoluble silver thiocyanate, there would be no permanent end-point.

2. Silver bromide and silver iodide are less soluble than silver thiocyanate, so that in the determination of bromine and iodine by this method it is not necessary to filter.

3. Soluble chlorates, etc., may be determined by this method by first reducing them to the corresponding halides (e.g. with sulphurous acid), and then determining the latter. 
4. For a simple rapid method for the determination of halogen in organic substances, with the final use of Volhard's procedure, see W. A. Van Winkle and G. McP. Smith, Jour. Amer. Chem. Soc., vol. 42, pp. 333-47 (I920). 5. For other uses of precipitation methods, see Part V, Problems 90, 91,92 , and 93 . 


\section{PART ·IV}

\section{QUESTIONS}

\section{Exercises with the Balance.}

I. What is the purpose of weighing?

2. Explain the mechanical theory of the balance.

3. Give five conditions which must be satisfied by a good balance.

4. What conditions must be fulfilled in order that a balance may be considered properly adjusted for use?

5. How may the rest-point of a balance be determined? Illustrate.

6. Explain the following methods of weighing: (a) ordinary method; (b) weighing by the use of deflections; (c) weighing by transposition; (d) weighing by substitution.

7. Describe a procedure for the calibration of a set of weights.

8. Discuss the errors in weighing which may be due: (a) to inequalities in length in the beam arms; $(b)$ to the buoyancy of the atmosphere.

9. What is a desiccator? Explain why it is necessary, and give the principles upon which its use is based.

\section{The Determination of Chlorine.}

I. What substances would, if present, interfere with this determination?

2. Why should the solution be acidified with nitric acid? Why should a large excess of nitric acid be avoided?

3. Why should the solution not be heated until after the addition of the silver nitrate? Why is it then heated?

4. What are the advantages of washing by decantation? In washing a precipitate, whether by decantation or otherwise, why should the liquid each time be removed as far as possible before the addition of fresh wash liquid?

5. How can you tell when the precipitate has been sufficiently washed?

6. Why is the filter paper ignited separately from the bulk of the precipitate? What is the object of the treatment with nitric and with hydrochloric acid? Explain.

7. What is the effect of light upon silver chloride? Is the action of diffused daylight a serious source of error? 
8. Why should the precipitate be heated until it just begins to fuse? What is the effect of overheating? Of underheating?

9. Water saturated with silver chloride at $100^{\circ}$ contains about $22 \mathrm{mg}$. of the salt per liter. Explain why the precipitate may be thoroughly washed with hot water without undue loss. What is the solubility of silver chloride in water at the ordinary room temperature? In the precipitation of chlorideion, why should silver nitrate be added in moderate excess?

Io. Given an aqueous solution of silver chloride in equilibrium with a quantity of the solid salt: What would happen upon the addition of $(a)$ a few drops of silver nitrate solution? (b) a few drops of sodium chloride solution? (c) a large excess of sodium chloride? Explain in each case.

II. How may the crucible safely and readily be cleaned after the ignition of the silver chloride? Write equations to show the reactions involved.

12. (a) Explain the solubility of silver chloride in each of the following substances: aqueous ammonia; potassium cyanide solution; sodium thiosulphate solution. (b) Is silver iodide soluble in aqueous ammonia? In a solution of potassium cyanide? Explain your answers.

13. What other substances may be determined in a similar manner in the form of insoluble silver salts?

I4. Why are Gooch crucibles preferable to ordinary paper filters; especially, for example, in the determination of iodides, cyanides, etc.?

I5. Starting with the native mineral, describe the treatment which renders the asbestos suitable for use in the preparation of Gooch crucibles.

\section{The Determination of Iron and of Sulphur in a Soluble Sulphate of Iron}

Iron. - I. A mixture consists of ferric sulphate, sodium carbonate, and potassium sulphate, each of which is soluble in cold water. Will the mixture dissolve in water? Illustrate your answer by means of equations.

2. How is the sample taken into solution for this analysis?

3. In the precipitation with ammonium hydroxide, why must the iron be present wholly in the ferric condition?

4. How is the ferrous iron oxidized in this analysis? Write the equation. How can you tell when the oxidation is complete?

5. How may it be ascertained whether the original sample contains ferrous iron? Is it worth while to make this test? Why?

6. Explain in full the principle involved in the use of double precipitation for effecting the complete separation of sulphate-ion from the ferric iron.

7. Why is the filter paper macerated with the solution before the second precipitation of the iron? 
8. Is it necessary to completely wash out the ammonium chloride before the ferric hydroxide is ignited? Why?

9. What precautions are to be observed in igniting the precipitate of ferric hydroxide?

ro. Name two other metals which may be determined by a similar procedure. What additional precautions should be taken in their determination, and why?

II. In the determination of these three metals, would it be equally well to use sodium hydroxide as the precipitating reagent? Give the reason for your answer in the case of each metal.

12. What is the effect of tartaric or citric acid, sugars, etc. upon the precipitation of ferric hydroxide by means of ammonium or sodium hydroxide? Do any other metallic ions behave like $\mathrm{Fe}^{+++}$-ion in this respect? Explain.

Sulphur. - r. Why must nitrates be removed before the precipitation with barium chloride? How is this done? Write the reaction.

2. Name some other substances which, if present, should be removed before precipitation with barium chloride.

3. Why is barium chloride chosen as the reagent for sulphate, rather than lead nitrate or strontium chloride?

4. How many cubic centimeters of I-normal barium chloride solution will be required to precipitate the sulphate from one gram of a sample containing $60 \%$ of $\mathrm{Fe}_{2}\left(\mathrm{SO}_{4}\right)_{3}$ and $\mathrm{I}_{4} \%$ of $\mathrm{K}_{2} \mathrm{SO}_{4}$ ?

5. What are the correct conditions for the precipitation of the sulphate? Explain in full.

6. What precautions should be observed in the ignition of the barium sulphate, and why?

7. What other substances may be determined as insoluble sulphates? What reagent is used in their precipitation?

\section{The Determination of Sulphur in a Sulphide Ore.}

I. Assuming complete oxidation, write an equation to show the action of nitric acid upon iron pyrites, $\mathrm{FeS}_{2}$.

2. If pure $\mathrm{FeS}_{2}$ were decomposed in this way with nitric acid, the iron precipitated with ammonia, and the filtrate evaporated with hydrochloric acid on the hot plate to dryness (to remove the nitrates), would there be any danger of losing a portion of the sulphur? Explain.

3. Explain the solubility of lead chloride in ammonium chloride solution. (Cf. the behavior of silver chloride.)

4. In the analysis of an ore containing lead, how may we prevent the precipitation of a portion of the sulphur as lead sulphate? Fully explain your answer. 
5. Outline an experimental procedure for the determination of sulphur in heavy spar, $\mathrm{BaSO}_{4}$.

\section{The Determination of Potash.}

I. Is this a precipitation method? If not, to what class does it belong?

2. Why is it necessary to remove hydrochloric and sulphuric acids before the extraction of the soluble perchlorates? How are these acids removed? Explain.

3. What weight of $\mathrm{HClO}_{4}$ will be required to convert one gram of $\mathrm{NaCl}$ into $\mathrm{NaClO}_{4}$ ? One gram of $\mathrm{KCl}$ into $\mathrm{KClO}_{4}$ ? What connection exists between these questions and the procedure? (Cf. Part V, Problem 30.)

4. Explain the fact that phosphates, even if insoluble in alcohol, need not be removed before making the extraction. What precaution should be observed when phosphates are present, and why?

5. Do any of the following salts interfere with the method; and, if so, how is the difficulty overcome? $\mathrm{NH}_{4} \mathrm{ClO}_{4}, \mathrm{Ba}\left(\mathrm{ClO}_{4}\right)_{2}, \mathrm{Mg}\left(\mathrm{ClO}_{4}\right)_{2}$.

6. Why is a small quantity of $\mathrm{HClO}_{4}$ added to the alcohol to be used in the extraction? If $50 \mathrm{cc}$. of this liquid, capable of dissolving $2 \mathrm{mg}$. of pure $\mathrm{KClO}_{4}$, are used in the extraction, why is not the quantity of $\mathrm{KClO}_{4}$ found in the residue $2 \mathrm{mg}$. less than the true value?

7. How may we determine the point at which the extraction is complete? Explain.

\section{The Analysis of Limestone.}

r. What is the principal component of limestone? What other compounds are usually present? Why is it important to analyze a limestone?

2. Explain briefly the method described in the procedure for the determination of carbon dioxide.

3. What are the objections to this method? Under what conditions can the method be relied upon to furnish accurate results?

4. What modifications should be made in the procedure if the sample to be analyzed is a baking powder?

5. Explain the operation of the aspirator. Why is the carbon dioxide more readily removed when air is bubbled through the solution? (Cf. Part I, The Evaporation of Liquids.)

6. What method for the determination of carbon dioxide may be regarded as the converse of the method described in the procedure? Describe it.

7. Can you think of any other method that might serve for the exact determination of carbon dioxide? (Cf. Part V, Problem 33.) 
8. In preparing a solution of the limestone, for the determination of calcium oxide, etc., what is the reason for the double evaporation with hydrochloric acid, followed by continued heating on the steam bath? When this residue is extracted with hydrochloric acid, what is the insoluble material that is left?

9. Why is the insoluble residue first washed with dilute acid rather than with water? Would cold water do about as well? Why?

Io. What is the purpose of adding bromine water in the precipitation with ammonium hydroxide? Why must a large excess of ammonia be avoided?

II. Why should the ammonium hydroxide precipitate be filtered off promptly? Of what does this precipitate consist? How is this precipitate treated, and why?

12. How is the first ammonium oxalate precipitate treated, and why?

13. Explain why so little ammonium oxalate solution is added in the second precipitation of the calcium.

I4. Explain the solubility of calcium oxalate in hydrochloric acid and its insolubility in acetic acid.

I5. What reactions take place when calcium oxalate, $\mathrm{CaC}_{2} \mathrm{O}_{4} \cdot \mathrm{H}_{2} \mathrm{O}$, is ignited?

I6. Why should the solution be only faintly ammoniacal for the precipitation of the magnesium? Why is more ammonia later added to the precipitation mixture? Why is the precipitation mixture then allowed to stand for several hours before filtration?

17. How is the first precipitate of magnesium ammonium phosphate treated? Explain why this is necessary. Is it possible to accomplish the same result in any other way, and if so how?

18. Why is the precipitate washed with dilute ammonia rather than with water?

19. Write an equation to show what happens when $\mathrm{MgNH}_{4} \mathrm{PO}_{4} \cdot 6 \mathrm{H}_{2} \mathrm{O}$ is ignited; when $\mathrm{Mg}\left[\left(\mathrm{NH}_{4}\right)_{2} \mathrm{PO}_{4}\right]_{2} \cdot n \mathrm{H}_{2} \mathrm{O}$ is ignited.

20. What precautions should be observed in igniting the precipitate, and why?

\section{The Determination of Phosphoric Anhydride in Phosphate Rock.}

I. What are the chief components of phosphate rock? What other compounds are usually present? What is apatite?

2. Explain by means of the ionic theory and the solubility product law the fact that calcium phosphate, $\mathrm{Ca}_{3}\left(\mathrm{PO}_{4}\right)_{2}$, will dissolve in nitric acid.

3. Why is it necessary to remove any soluble silicic acid that may be present in the nitric acid solution of the mineral? How is this done? 
4. Explain why it is possible, in washing the insoluble residue of silica, to make the test for phosphate in the washings by the addition of ammonia alone.

5. Why is it directed to neutralize the nitric acid solution with ammonia and then to make it slightly acid with nitric acid, before the addition of the molybdate reagent?

6. Why is an acid solution of ammonium nitrate used in washing the yellow precipitate? (Cf. question 9.) What would happen if this ammonium nitrate wash-liquid were alkaline with ammonia? Explain.

7. Could the phosphorus be determined by igniting and weighing the yellow precipitate?

8. Why do we first precipitate the phosphate with molybdate solution instead of precipitating it directly from the original solution with magnesia mixture?

9. Explain why a large excess of the molybdate reagent is necessary for the complete precipitation of the phosphate.

ro. Show by means of an equation the reaction between ammonium phosphomolybdate, $\left(\mathrm{NH}_{4}\right)_{3} \mathrm{PO}_{4} . \mathrm{I}_{2} \mathrm{MoO}_{3}$, and ammonium hydroxide. Sodium hydroxide.

I1. What is magnesia mixture? What is the purpose of the ammonium chloride? Explain.

12. Why should the magnesium ammonium phosphate precipitation mixture be allowed to stand for some hours before filtering?

13. Why is dilute ammonia used in washing the precipitate of magnesium ammonium phosphate? Why not use water?

I4. What precautions should be observed in the ignition of this precipitate, and why?

I5. Name another substance which may be determined by precipitation with magnesia mixture. Can any elements be determined in a similar manner by precipitation with sodium or ammonium phosphate? If so, name them.

I6. If it were desired to determine the phosphorus in a sample of steel, in which it is present as iron phosphide, what would be the procedure? Explain.

\section{The Determination of Silica in a Refractory Silicate.}

I. Why is it essential to grind the whole of the sample very fine?

2. Write an equation to show the reaction between orthoclase, $\mathrm{KAlSi}_{3} \mathrm{O}_{8}$, and sodium carbonate, above the melting point of the latter. Why is a very large excess of the latter used?

3. How can you tell whether the decomposition is complete $(a)$ by in- 
specting the mixture during fusion; (b) after the treatment of the melt with dilute acid?

4. Why is the fused mass treated with a considerable volume of dilute acid, rather than with concentrated acid?

5. How is the silica separated from the residue left upon evaporation with dilute hydrochloric acid? Explain.

6. What is the purpose of treating the silica with hydrofluoric and sulphuric acids? Explain in full.

7. How would you determine the percentage of mixed iron and aluminum oxides in an insoluble silicate? How would you determine the calcium oxide? The magnesium oxide?

8. How would you determine the silica in a silicate mineral which may be readily decomposed by hydrochloric acid?

\section{The Electrolytic Determination of Copper.} laws.

I. Define: ampere; volt; ohm. State Ohm's law. State Faraday's

2. Show by means of a diagram how the apparatus is assembled for an electrolytic determination.

3. Explain the electrolytic reduction of nitric acid to ammonia.

4. Explain the deposition of lead peroxide upon the anode, in the electrolysis of a solution containing lead nitrate and nitric acid.

5. What is meant by the discharge potential of an ion? The decomposition voltage of a salt? What is polarization? Explain in each case.

6. Explain why it is possible to separate copper from nickel by electrolysis? Can nickel be separated from cobalt in this way? Why?

7. What are the advantages of mechanical stirring during electrolysis?

8. What is meant by current density? What is the unit of current density? Why is current density a factor of the greatest importance in electro-analysis?

9. What factors favor the formation of a satisfactory deposit? What factors interfere with it?

Io. Outline a method for the preparation of a solution suitable for electrolysis when the sample to be analyzed is a copper ore containing arsenic. Why should the arsenic be removed?

II. Discuss the materials from which electrodes may be prepared, and the form of the electrodes and electrolytic vessels.

I2. Name the factors in the electrolytic work which should receive especial attention in an endeavor to make accurate and reliable copper determinations as rapidly as possible. Explain in the case of each factor mentioned. 


\section{Volumetric Analysis: Fundamental Principles.}

I. Specify the chief uses of measuring flasks; of transfer pipettes; of burettes.

2. Describe the preparation of sulphuric acid-dichromate cleaning solution. How is it used?

3. In a titration with a solution correctly made up to tenth-normal concentration at $20^{\circ}$, a burette correctly graduated for use at $20^{\circ}$ is used at an actual temperature of $27.5^{\circ}$, and the indicated volume of solution withdrawn is $27.68 \mathrm{cc}$; to how many cubic centimeters of tenth-normal solution does this liquid correspond? (See Part I.)

4. Define the term "liter."

5. Describe a method for the calibration of a roo-cc. measuring flask. Illustrate your description. (See Part I.)

6. What is a standard solution? A normal solution? Define and illustrate the term "normality factor."

7. Characterize in general the reactions upon which volumetric processes may be based.

8. What is an indicator? Illustrate your answer.

9. Discuss the advantages of the volumetric system.

Neutralization Methods: The Standardization of Acids and Alkalies.

I. Define in terms of the theory of ionization: (a) a neutral solution; (b) an acid solution; (c) an alkaline solution.

2. Will the solution resulting from mixing equal volumes of one tenth normal aqueous solutions of the following substances be acid, alkaline, or neutral: ( $a$ ) hydrogen chloride and sodium hydroxide; (b) hydrogen chloride and ammonium hydroxide; $(c)$ acetic acid and sodium hydroxide?

3. Give a full explanation of case $(c)$ above, writing all equations and equilibria.

4. What indicator should be used in each case in titrations involving the combinations indicated in question 2? Explain fully. (See the section on Indicators for Use in Alkalimetry and Acidimetry.)

5. Outline procedures for the preparation of approximately half-normal solutions of hydrochloric acid and sodium hydroxide.

6. Describe a method for obtaining the ratio between the solutions referred to in the preceding question. If $20.00 \mathrm{cc}$. of an acid solution require $2 \mathrm{I} .46 \mathrm{cc}$. of 0.4693 -normal alkali-for neutralization, what is the normality factor of the acid?

7. Describe the sodium carbonate method for the standardization of a solution of hydrochloric acid. If $0.5383 \mathrm{~g}$. of $\mathrm{Na}_{2} \mathrm{CO}_{3}$ require $20.15 \mathrm{cc}$. of the acid for neutralization, what is the normality factor of the acid? 
8. If $10.00 \mathrm{cc}$. of a solution of hydrogen chloride yield $0.742 \mathrm{I} \mathrm{g}$. of silver chloride, what is the normality factor of the acid?

9. Describe the standardization of sodium hydroxide solution by means of potassum bitartrate. If $\mathrm{r} .179 \mathrm{~g}$. of the latter require $\mathrm{I3.35} \mathrm{cc}$. of the alkali for neutralization, what is the normality factor of the solution?

10. If $20.00 \mathrm{cc}$. of an acid are equivalent to $21.20 \mathrm{cc}$. of an alkali, and if $40.00 \mathrm{cc}$. of the acid are added to $0.6000 \mathrm{~g}$. of $\mathrm{Na}_{2} \mathrm{CO}_{3}$ and the resulting solution requires $3.00 \mathrm{cc}$. of the alkali for neutralization, what are the normality factors of the two solutions?

II. Describe a method for the preparation of a solution of hydrochloric acid of exactly one half normal concentration.

\section{The Alkaline Value of Soda.}

I. Discuss the composition of crude soda, and account for the impurities it is likely to contain.

2. Which of the impurities will contribute to the alkaline strength of the soda?

3. Could this determination be made with phenolphthalein as the indicator, and if so how?

4. Give two volumetric methods for the determination of $\mathrm{Na}_{2} \mathrm{CO}_{3}$ and of $\mathrm{NaOH}$ in mixtures of the two substances.

5. Give a method for the determination of $\mathrm{Na}_{2} \mathrm{CO}_{3}$ and of $\mathrm{NaHCO}_{3}$ in mixtures of the two salts.

\section{The Available Hydrogen-ion in an Acid.}

I. In titrating weak acids, why is it usually necessary to work at the boiling temperature?

2. At what point does an ice-cold, dilute solution of sodium hydroxide containing phenolphthalein lose its red color upon being treated with carbon dioxide? Upon boiling this decolorized solution, the color reappears; explain fully the mechanism by which the alkalinity of the solution increases on boiling.

3. Describe a method for the preparation of a carbonate-free solution of sodium hydroxide. How should such a solution be preserved? Why is its preparation sometimes worth while?

4. Is it possible to accurately titrate sulphurous acid with a standard solution of ammonia? If not, why? And if so, what indicator should be used?

\section{The Determination of Protein Nitrogen by the Kjeldahl Method.}

r. What is the purpose in the digestion of $(a)$ the concentrated sulphuric acid? (b) The copper sulphate? (c) The potassium sulphate? 
2. What chemical change does sulphuric acid undergo during the digestion? The organic matter?

3. Why is a long-necked flask used?

4. How would the procedure of digestion be modified if nitrates were present? Explain fully.

"5. How would the procedure be modified if mercury were added instead of copper sulphate? Explain in full.

6. In what form does the nitrogen exist after the completion of the digestion? After making the solution alkaline with sodium hydroxide? In what form does it distill over?

7. How is the loss of ammonia prevented upon the addition of an excess of sodium hydroxide?

8. What is the purpose of the zinc? What is the action of sodium hydroxide solution upon zinc?

9. The ammonia from one gram of a fertilizer is distilled into $20.00 \mathrm{cc}$. of $0.5000 \mathrm{~N}$ acid, and $6.00 \mathrm{cc}$. of $0.4800 \mathrm{~N}$ alkali are required to neutralize the excess of acid; calculate the percentage of nitrogen in the sample.

I0. If $0.20 \mathrm{cc}$. of $25.00 \% \mathrm{NaOH}$ (sp. gr., I.25) had been carried over by bumping or foaming, what would have been the apparent percentage of nitrogen in the above case?

II. If you had to determine the percentage of $\mathrm{NH}_{3}$ and of $\mathrm{HC}_{2} \mathrm{H}_{3} \mathrm{O}_{2}$ in crude ammonium acetate, how would you proceed?

\section{Dichromate Methods: The Titration of Iron.}

I. Write the equation for the oxidation of ferrous chloride with potassium dichromate in the presence of hydrochloric acid.

2. What weight of $\mathrm{K}_{2} \mathrm{Cr}_{2} \mathrm{O}_{7}$ is required for one liter of a tenth-normal solution, to be used as an oxidizing agent?

3. Outline the procedure for the standardization of dichromate solution by means of iron wire. Why is it well also to have a standard solution of ferrous ammonium sulphate, and how is this solution standardized?

4. What is the maximum weight of pure iron wire which can be taken for reaction with tenth-normal dichromate without having to refill a 30-cc. burette?

5. Name four reagents which can be used to reduce ferric salts to ferrous, in the presence of hydrochloric acid, and write the equation in each case.

6. Why is it necessary after reduction with stannous chloride to add mercuric chloride to the solution? Why must the stannous chloride be present only in very slight excess (equations)? Why should hydrochloric acid be present during the titration? 
7. What indicator is used in the titration? What action has it upon ferric salts? Upon ferrous salts?

8. Why is the indicator used outside of the solution?

9. Can you give a method of treatment for chrome iron ore which might lead to a determination of its chromium content by means of standard solutions of ferrous iron and dichromate?

\section{Permanganate Methods: The Titration of Iron and of Oxalic Acid.}

I. How many grams of $\mathrm{KMnO}_{4}$ are required for one liter of tenth-normal solution, to be used as an oxidizing agent in acid solutions? To be used in neutral solutions for the oxidation of manganese?

2. Why should a permanganate solution be allowed to stand for several days, and then be filtered through asbestos, before it is standardized? Why should it not be placed in burettes having rubber outlet tubes?

3. Name at least four substances which can be used to standardize permanganate solutions. Write an equation in each case.

4. What is the maximum weight of $\mathrm{Na}_{2} \mathrm{C}_{2} \mathrm{O}_{4}$ which can be titrated with tenth-normal permanganate solution without having to refill a $30-c c$. burette?

5. In the titration of oxalic acid, why is it that the oxidation proceeds so much more slowly at first than later on? Explain in full.

6. Name ten substances which can be quantitatively determined by means of potassium permanganate.

7. What effect does potassium permanganate have upon hydrochloric acid in the presence of ferrous salts, even in very dilute solution? How may this action be prevented?

8. What are the components of the Zimmermann-Reinhardt solution? Explain the purpose of each.

9. Under what conditions can ferrous iron be accurately determined with potassium permanganate without the use of the Zimmermann-Reinhardt solution?

I0. What is the maximum weight of a sample of ore containing $40.00 \%$ of iron which can be taken for titration with tenth-normal oxidizing agent without having to refill a 30-cc. burette?

II. Discuss the preparation of a solution for analysis from a refractory iron ore.

I2. Describe a method for the determination of calcium by means of potassium permanganate.

13. Outline a method for the determination of the $\mathrm{MnO}_{2}$-value of pyrolusite by means of potassium permanganate.

14. How can the determination referred to in the preceding question be made by a gravimetric process? 


\section{The Determination of Phosphorus in Steel.}

I. Assuming the presence of the phosphorus as $\mathrm{Fe}_{3} \mathrm{P}_{2}$, show by means of an equation the action of nitric acid upon this compound. Why is the nitric acid solution heated with potassium permanganate?

2. In order to cause the higher oxides of manganese, such as $\mathrm{MnO}_{2}$, to go into solution in nitric acid, what kind of a reagent should be added? Illustrate and explain.

3. What is the purpose of precipitating the phosphorus as ammonium phosphomolybdate?

4. Why is the yellow precipitate dissolved in ammonia and the solution acidified with sulphuric acid, rather than to dissolve it directly in sulphuric acid? Why is $\mathrm{MoO}_{3}$ not precipitated when the ammoniacal solution is acidified with sulphuric acid? Would hydrochloric or nitric acid do as well here, and why?

5. Describe the construction and use of the Jones reductor. Can it be used for the reduction of substances other than molybdenum?

6. Why is it best to receive the reduced molybdenum solution below the surface of a solution containing ferric alum? What is the purpose of the phosphoric acid in this solution?

7. How should the permanganate solution be standardized in order to obtain the most reliable results?

8. Would you recommend the determination of phosphoric anhydride in apatite by this method? Why? Is there a suitable volumetric method? If so, describe it.

\section{The Determination of Manganese in an Ore.}

I. What is the fundamental reaction of this process? What rôle does it play in the titration of oxalic acid or of iron with potassium permanganate?

2. Discuss the preparation of the solution for analysis from a refractory ore containing manganese.

3. What happens when zinc oxide is added to the acid solution of the ore (equations)?

4. Explain fully why a zinc salt should be present in the solution during the titration.

5. Explain why the presence of chlorides does not interfere with the accuracy of this titration.

6. If the permanganate solution used in this titration is $0.1000-N$ for use with iron or oxalic acid, what is its normality factor for this reaction?

7. How is it best to standardize the permanganate solution used in this determination? 


\section{Iodometric Methods: The Preparation and Standardization of Iodine and Thiosulphate Solutions.}

I. How many grams of iodine are required for one liter of the tenthnormal solution? Of sodium thiosulphate, $\mathrm{Na}_{2} \mathrm{~S}_{2} \mathrm{O}_{3} \cdot{ }_{5} \mathrm{H}_{2} \mathrm{O}$ ?

2. How is the iodine solution made, and what is the purpose of the potassium iodide? Show what equilibria exist in the iodine solution. Why should the water be freshly boiled and allowed to cool out of contact with the air, in the preparation of the thiosulphate solution?

3. Write the reaction between iodine and sodium thiosulphate. How do chlorine and bromine differ from iodine in their behavior towards sodium thiosulphate? Explain why this is so.

4. What is the effect of free carbonic acid upon sodium thiosulphate solution? Does the decomposition cease as soon as all of the carbonic acid has reacted? Write equations to illustrate your answers.

5. Does the solution resulting from the partial decomposition of the thiosulphate have a greater or lower reducing value than the original solution? Explain why.

6. Give equations to show two ways in which iodine may act as an oxidizing agent.

7. What is the maximum weight of $\mathrm{As}_{2} \mathrm{O}_{3}$ which can be taken for reaction with tenth-normal iodine solution without having to refill a 30-cc. burette?

8. Can the standardization of iodine against arsenious oxide be performed in a strongly alkaline solution? Can it be done in an acid solution? Give reasons for your answers.

9. What is the purpose of the sodium bicarbonate? Is the bicarbonate solution acid, alkaline, or neutral? Explain your answer.

Io. Discuss the determination of the end-point. Explain why the indicator is added in such large quantity.

II. Discuss the use of iodine solutions in the presence of sulphuric acid. In the presence of ammonium salts.

The Determination of Antimony in Stibnite.

I. Write the reaction between pure stibnite and hydrochloric acid.

2. Why must the hydrochloric acid solution be heated on the steam bath? Why must it not be boiled until after dilution?

3. What is the purpose of adding tartaric acid to the solution? Explain.

4. Explain why the solution may possibly turn red during gradual dilution. What is the correct procedure in such a case?

5. If a white precipitate forms upon dilution, what error has been made? What is the white precipitate, and what should be done with the mixture? 
6. What is the purpose of almost neutralizing the solution with sodium hydroxide, and how is this accomplished? Why is sodium bicarbonate then added in large excess?

7. What elements would, if present, interfere with this determination, and why?

\section{The Determination of Chromium in Chromite.}

r. What is the action of fused sodium peroxide upon ferrous oxide? Upon chromic oxide?

2. What happens upon the addition of water to the cooled chromitesodium peroxide fusion mixture?

3. Why is it necessary to completely decompose the excess of the peroxide before acidifying the aqueous extract?

4. Give two processes for the volumetric determination of chromium in the acidified extract, and explain each.

\section{The Determination of Lead in an Ore.}

I. After the decomposition of the ore and the addition of sulphuric acid, why is it necessary to evaporate to white fumes?

2. Explain the solubility of lead sulphate in ammonium acetate solution.

3. Explain why it is possible to quantitatively precipitate the lead from the ammonium acetate solution by means of an excess of potassium dichromate.

4. Explain the solubility of lead chromate in the acidified solution of sodium chloride.

5. Write an equation to show the reaction of the acid chromate solution with potassium iodide.

6. Why should the thiosulphate solution used in this determination be standardized under identical conditions against test lead?

\section{The Determination of Copper in an Ore.}

I. In this determination, why is the copper separated from the other metals present in the ore? Explain how iron, arsenic, or antimony would interfere with the accuracy of the titration.

2. What would you expect the composition of the precipitate to be which is formed upon the addition of sodium thiosulphate to a solution of copper sulphate? How does it happen, then, that we obtain cuprous sulphide?

3. Can any other metals be precipitated from their salt solutions by means of sodium thiosulphate? (Try, for example, silver nitrate and sodium thiosulphate, in the cold, and explain what takes place.) 
4. What is the object of igniting the precipitate of cuprous sulphide? What becomes of any antimony which is present?

5. Why is it so important to standardize the thiosulphate solution against pure metallic copper?

6. Why must the nitrous fumes be completely expelled before the addition of the potassium iodide?

7. Why is it preferable to titrate the free iodine in the presence of acetic acid, rather than in the presence of sulphuric acid? Explain fully.

8. Write an equation to show the action of nitric acid upon cuprous iodide.

\section{Precipitation Methods: The Determination of Chlorine.}

I. Briefly outline the procedure for the standardization of the silver nitrate and potassium thiocyanate solutions against pure sodium chloride. Could these solutions be standardized against pure metallic silver, and if so how?

2. How may pure sodium chloride be prepared from the commercial salt?

3. What indicator is used in connection with thiocyanate solutions? Why must nitric acid be present? Explain fully why the indicator should be added in such large quantity.

4. At $\mathrm{I} 8-20^{\circ}$, the solubility product of silver chloride is about $0.6 \times 10^{-10}$ and that of silver thiocyanate is about $0.6 \times 1 \mathrm{IO}^{-12}$; what is the relative concentration of the chloride and thiocyanate ions in a solution which is saturated with both salts? Assume the equal (practically complete) ionization of both salts.

5. Why is it necessary to filter off the silver chloride before making the titration with the thiocyanate solution? Base your explanation upon the data given in the preceding question.

6. In general, what anions may be determined by this method without first filtering off the silver salt?

7. How may the halogens in alkali chlorates, bromates, and iodates be determined by this method?

8. Outline a procedure for the determination by this method of $(a)$ the chlorine in horn silver, $\mathrm{AgCl}$; (b) the silver in the same mineral. 


\section{PART V}

\section{ANALYTICAL PROBLEMS}

Preliminary Discussion. In spite of any preconception to the contrary, the calculations involved in analytical work are not difficult, and a reasonable amount of application will enable the student to master the subject. At the same time, the study of the following problems will furnish an insight into the principles of a wide variety of processes.

It cannot be too strongly emphasized that, in making analytical calculations, the beginner should from the outset strive to take the shortest and most direct route to the result. With a little practice, the student who is not unacquainted with the reactions of analytical chemistry should soon be able upon the inspection of a problem to promptly recognize the factors which will lead most directly to its solution, ${ }^{1}$ as well as the equivalent relationships of the substances involved. To do this, however, he should

${ }^{1}$ Of course most analytical problems can be solved in stages, by means of a series of proportions, and it is perhaps only natural that most beginners should have a predilection for this method. In the examples given, however, the common factors have been eliminated, and the problems solved in a single operation. The beginning student will better appreciate the advantages of the shorter method upon comparing the solutions given of problems iv and $v$ with the following roundabout method of arriving at the same results :

iv.

ro $\mathrm{Fe}: 2 \mathrm{KMnO}_{4}=0.005: w$. $w=316 / 558 \times 0.005=0.00283 \mathrm{~g}$. $\mathrm{KMnO}_{4}$ per cc. $2 \mathrm{KMnO}_{4}: 5 \mathrm{H}_{2} \mathrm{C}_{2} \mathrm{O}_{4}=0.00283: x$. $x=450 / 3 \mathrm{r} 6 \times 0.00283=0.00403 \mathrm{~g} . \mathrm{H}_{2} \mathrm{C}_{2} \mathrm{O}_{4}$ per cc. $5 \mathrm{H}_{2} \mathrm{C}_{2} \mathrm{O}_{4}: 5 \mathrm{CaC}_{2} \mathrm{O}_{4}=0.00403: y$. $y=640 / 450 \times 0.00403=0.00573 \mathrm{~g} . \mathrm{CaC}_{2} \mathrm{O}_{4}$ per cc. $z=280.5 / 640.5 \times 0.00573=0.00251 \mathrm{~g} . \mathrm{CaO}$ per cc. Ans. 
understand and bear in mind the relationships and differences which exist between chemical and physical units, - such as atoms, molecules, and equivalents on the one hand, and grams and cubic centimeters on the other.

In order to illustrate what is meant, the following problems are given, with their solutions.

i. A sample of a soluble chloride weighing $0.2007 \mathrm{~g}$. yields on analysis $0.4920 \mathrm{~g}$. of silver chloride; what percentage of chlorine does it contain?

From the proportion,

Wt. of chlorine in sample: Wt. of sample $=\%$ of chlorine : 100 , it is obvious that $\frac{\mathrm{Wt} \text {. of chlorine in sample }}{\mathrm{Wt} \text {. of sample }} \cdot 100=\%$ of chlorine. Also, since the chlorine contained in the sample is identical with that which is later contained in the silver chloride precipitate, we have the proportion,

$\mathrm{Cl}: \mathrm{AgCl}=\mathrm{Wt}$. of chlorine: Wt. of silver chloride, or, $\quad \frac{\mathrm{Cl}}{\mathrm{AgCl}} \cdot \mathrm{Wt}$. of silver chloride $=\mathrm{Wt}$. of chlorine. Substituting this value in the preceding equation, we get,

$$
\begin{aligned}
& \frac{\frac{\mathrm{Cl}}{\mathrm{AgCl}} \cdot \text { Wt. of silver chloride }}{\text { Wt. of sample }} \cdot 100=\frac{\frac{35.46}{\mathrm{I} 43.34} \cdot 0.4920}{0.2007} \cdot 100 \\
& =60.50 \% \text { of } \mathrm{Cl} \text {. }
\end{aligned}
$$

A chemical factor represents the quantity by weight of an element or compound which is equivalent to one part by weight of some other substance. For example, the ratio or factor

$$
\frac{\mathrm{Ag}}{\mathrm{AgCl}}=\frac{\mathrm{I} 07.88}{\mathrm{I} 43.34}=0.7526
$$

ข.

(a) $\mathrm{AgCl}: \mathrm{HCl}=0.1527: x$.

$x=36.46 / 143.34 \times 0.1527=0.0388 \mathrm{~g} . \mathrm{HCl}$ in $20.50 \mathrm{cc}$.

(b)

$20.50: 1000=0.0388: y$.

(c) $y=1000 / 20.5 \times 0.0388=1.893 \mathrm{~g} . \mathrm{HCl}$ in one liter.

$36.46: \mathrm{r} .893=\mathrm{r}: z$. $z=1.893 / 36.46=0.0519 \mathrm{~N}$. Ans. 
tells us that one gram of silver chloride contains $0.75^{26} \mathrm{~g}$. of silver, and if we wish to calculate what weight of silver there is in a specific weight of silver chloride, we simply multiply the latter by this factor; e.g. I0.15 g. of silver chloride contain I0.I $5 \times 0.75^{26}=7.64 \mathrm{~g}$. of silver.

Or, if the weight of $\mathrm{MnO}$ is wanted which corresponds to a definite weight of $\mathrm{Mn}_{3} \mathrm{O}_{4}$, the factor is

$$
\frac{3 \mathrm{MnO}}{\mathrm{Mn}_{3} \mathrm{O}_{4}}=\frac{2 \mathrm{I} 2.79}{228.79}=0.930 \mathrm{I} \text {. }
$$

And if we wish to find the weight of $\mathrm{K}_{2} \mathrm{O}$ which corresponds to a given weight of $\mathrm{KClO}_{4}$, we multiply the latter by the factor,

$$
\frac{\mathrm{K}_{2} \mathrm{O}}{2 \mathrm{KClO}_{4}}=\frac{94.20}{277.12}=0.3399 \text {. }
$$

Since, in arriving at these (physical-unit) factors, our calculations must be based upon chemical facts, it is necessary to keep strictly to the equivalent relations of the substances. Thus, in the last example, since every molecule of $\mathrm{K}_{2} \mathrm{O}$ would be converted upon evaporation with perchloric acid into two molecules of $\mathrm{KClO}_{4}$, we must use one mol of $\mathrm{K}_{2} \mathrm{O}$ in the numerator and two of $\mathrm{KClO}_{4}$ in the denominator. Any other ratio would be incorrect.

ii. How many cubic centimeters of a solution containing $\mathbf{2} 2.5$ grams of $\mathrm{BaCl}_{2} .2 \mathrm{H}_{2} \mathrm{O}$ per liter will be required to precipitate the sulphur from 0.1073 gram of pure stibnite, $\mathrm{Sb}_{2} \mathrm{~S}_{3}$, as $\mathrm{BaSO}_{4}$ ?

Each molecule of $\mathrm{Sb}_{2} \mathrm{~S}_{3}$ will yield upon treatment three molecules of $\mathrm{H}_{2} \mathrm{SO}_{4}$, and these will react with three molecules of barium chloride. We therefore arrive at the proportion, $3\left(\mathrm{BaCl}_{2}\right.$. $\left.{ }_{2} \mathrm{H}_{2} \mathrm{O}\right): \mathrm{Sb}_{2} \mathrm{~S}_{3}=x: 0.1073$, where $x$ is the weight of the crystalline salt required. That is,

$$
x=\frac{3\left(\mathrm{BaCl}_{2} .2 \mathrm{H}_{2} \mathrm{O}\right)}{\mathrm{Sb}_{2} \mathrm{~S}_{3}} \cdot \text { o.1073, }
$$

in which the factor $\frac{3\left(\mathrm{BaCl}_{2} .2 \mathrm{H}_{2} \mathrm{O}\right)}{\mathrm{Sb}_{2} \mathrm{~S}_{3}}=2.177$ indicates the quan- 
tity by weight of $\mathrm{BaCl}_{2}, 2 \mathrm{H}_{2} \mathrm{O}$ which is required to precipitate the sulphur from one gram of $\mathrm{Sb}_{2} \mathrm{~S}_{3}$; this ratio therefore does not differ essentially from the chemical factors previously discussed.

Finally, since each cubic centimeter of the solution contains 0.0125 g. of $\mathrm{BaCl}_{2} \cdot 2 \mathrm{H}_{2} \mathrm{O}$, we have,

$$
\frac{\frac{3\left(\mathrm{BaCl}_{2} \cdot 2 \mathrm{H}_{2} \mathrm{O}\right)}{\mathrm{Sb}_{2} \mathrm{~S}_{3}} \cdot 0.1073}{0.0125}=\frac{\frac{732.9}{336.6} \cdot 0.1073}{0.0125}=18.68 \mathrm{cc} .
$$

iii. What volume of aqueous ammonia of sp. gr. 0.960, containing 9.9r \% of $\mathrm{NH}_{3}$, will be required to precipitate, as $\mathrm{Fe}(\mathrm{OH})_{3}$, the iron contained in $\mathrm{I} .475 \mathrm{~g}$. of $\mathrm{Fe}\left(\mathrm{NH}_{4} \mathrm{SO}_{4}\right)_{2} .6 \mathrm{H}_{2} \mathrm{O}$ ?

Since the iron is to be precipitated, after oxidation, as $\mathrm{Fe}(\mathrm{OH})_{3}$, we see that each atom of iron will require three molecules of $\mathrm{NH}_{4} \mathrm{OH}$, which in turn are furnished by three molecules of $\mathrm{NH}_{3}$. Therefore, $\frac{3 \mathrm{NH}_{3}}{\mathrm{Fe}\left(\mathrm{NH}_{4} \mathrm{SO}_{4}\right)_{2} \cdot 6 \mathrm{H}_{2} \mathrm{O}} \cdot \mathrm{I} \cdot 457=$ wt. of $\mathrm{NH}_{3}$ required; and, since each cubic centimeter of the aqueous ammonia weighs 0.960 g., and contains $9.91 \%$ of $\mathrm{NH}_{3}$, the solution contains $0.960 \times 0.099 \mathrm{I}$ g. of $\mathrm{NH}_{3}$ per cubic centimeter. That is,

$$
\frac{\frac{3 \mathrm{NH}_{3}}{\mathrm{Fe}\left(\mathrm{NH}_{4} \mathrm{SO}_{4}\right)_{2} \cdot 6 \mathrm{H}_{2} \mathrm{O}} \cdot \mathrm{I} .457}{0.960 \times 0.099 \mathrm{I}}=\frac{\frac{5 \mathrm{I} . \mathrm{IO}}{392 . \mathrm{I} 6} \cdot \mathrm{I} .475}{0.960 \times 0.099 \mathrm{I}}=2.02 \mathrm{cc} \text {. }
$$

iv. A solution of potassium permanganate is equivalent to $0.00500 \mathrm{~g}$. of ferrous iron per cubic centimeter; what is its value in terms of calcium oxide?

The reactions involved in the volumetric determinations of iron and calcium are:

Io $\mathrm{FeSO}_{4}+2 \mathrm{KMnO}_{4}+9 \mathrm{H}_{2} \mathrm{SO}_{4}=2 \mathrm{KHSO}_{4}+2 \mathrm{MnSO}_{4}$

and

$$
+5 \mathrm{Fe}_{2}\left(\mathrm{SO}_{4}\right)_{3}+8 \mathrm{H}_{2} \mathrm{O} \text {, }
$$

$5 \mathrm{CaC}_{2} \mathrm{O}_{4}+2 \mathrm{KMnO}_{4}+9 \mathrm{H}_{2} \mathrm{SO}_{4}=5 \mathrm{CaSO}_{4}+2 \mathrm{KHSO}_{4}$ $+2 \mathrm{MnSO}_{4}+10 \mathrm{CO}_{2}+8 \mathrm{H}_{2} \mathrm{O}$; 
and from these equations it is seen that, in this case,

$2 \mathrm{Fe}^{++} \approx \mathrm{CaO}$. That is,

$$
\frac{\mathrm{CaO}}{2 \mathrm{Fe}} \cdot 0.00500=\frac{56 . \mathrm{I}}{\text { III.6 }} \cdot 0.00500=0.0025 \mathrm{I} \mathrm{g} \cdot \mathrm{CaO} \text {. }
$$

v. If $20.50 \mathrm{cc}$. of hydrochloric acid yield $0.1527 \mathrm{~g}$. of silver chloride, what is the normality factor of the solution?

Although silver chloride is insoluble, the normality factor of the acid may nevertheless be calculated directly from the weight of the precipitate obtained. One liter of normal hydrochloric acid, containing one mol of $\mathrm{HCl}$, would yield r43.34 g. (i.e. one mol) of silver chloride, whence $20.50 \mathrm{cc}$. would yield 0.14334 $\times 20.50 \mathrm{~g}$. We obtain, therefore, the equation,

$$
\frac{0.1527}{0.14334 \times 20.50}=0.0519 N \text {. }
$$

vi. A sample of stibnite weighing $0.1793 \mathrm{~g}$. is heated with strong $\mathrm{HCl}$, and the $\mathrm{H}_{2} \mathrm{~S}$ evolved absorbed by means of sodium hydroxide solution; the resulting mixture (containing the sulphur as $\mathrm{Na}_{2} \mathrm{~S}$ ) being introduced under the surface of a solution made by adding $25 \mathrm{cc}$. of 6-normal $\mathrm{HCl}$ and $50.00 \mathrm{cc}$. of 0.II60-normal iodine to $500 \mathrm{cc}$. of water. The excess of iodine is titrated with $0.0957^{-}$ normal sodium thiosulphate solution, of which $28.57 \mathrm{cc}$. are required. Calculate the percentage of (evolved) sulphur in the stibnite. $\left(\mathrm{H}_{2} \mathrm{~S}+\mathrm{I}_{2}=2 \mathrm{HI}+\mathrm{S}\right.$. $)$

$$
\text { cc. N. F. }
$$

$50.00 \times 0.1160=5.800 \mathrm{cc}$. of normal iodine. $28.57 \times 0.0957=2.734 \mathrm{cc}$. of normal thiosulphate.

I.e. the $\mathrm{H}_{2} \mathrm{~S}$ required $3.066 \mathrm{cc}$. of normal iodine.

Since normal iodine has a sulphur value of $\frac{0.03207}{2}=0.016035 \mathrm{~g}$. per cubic centimeter, we have,

$$
\frac{0.016035 \times 3.066}{0.1793} \times 100=27.42 \% \text { of sulphur. }
$$


The normality factor of a solution expresses the value of the solution per cubic centimeter in terms of a normal solution. For example, if a solution is known to be one half normal (i.e. $\mathrm{N} . \mathrm{F} .=0.500$ ), it is obvious that I cc. of it is equivalent to $\mathrm{I} .000 \times 0.500=0.500 \mathrm{cc}$. of a normal solution; or that $27.3 \mathrm{I} \mathrm{cc}$. of it are equivalent to $27.31 \times 0.500=13.655 \mathrm{cc}$. of a normal solution. Knowing the normality factors of a series of solutions, therefore, we can readily reduce the different volumes of the solutions used in a determination to a common standard, and in this way render the calculations quite as simple as if the solutions had all been made up to, say, exactly one tenth normal.

vii. A mixture of potassium chloride and caesium chloride weighs $0.3895 \mathrm{~g}$. This is dissolved in water and the chlorine precipitated as silver chloride, of which there is obtained $0.4889 \mathrm{~g}$. Calculate the weight of each salt in the mixture.

Indirect methods of analysis depend upon the fact that when two or more substances are made to undergo the same chemical treatment they either experience a relatively different change of weight, or unit weights of each require unequal volumes of a standard solution.

Let $x$ represent the weight of the potassium chloride, in the above example, and $y$ that of the caesium chloride, $a$ the weight of the two salts, and $p$ that of the precipitate, and we have,

and

$$
x+y=a,
$$

$$
\frac{\mathrm{AgCl}}{\mathrm{KCl}} x+\frac{\mathrm{AgCl}}{\mathrm{CsCl}} y=p \text {. }
$$

If we designate by $m$ the factor $\frac{\mathrm{AgCl}}{\mathrm{KCl}}$ and by $n$ the factor $\frac{\mathrm{AgCl}}{\mathrm{CsCl}}$, we obtain, and

from which we find that

$$
\begin{array}{r}
x+y=a, \\
m x+n y=p,
\end{array}
$$

$$
\begin{aligned}
& x=\frac{p-n a}{m-n}, \\
& x=\frac{\mathrm{I}}{m-n} \cdot p-\frac{n}{m-n} \cdot a .
\end{aligned}
$$


Indirect analyses may in general be calculated by means of this or a similar general equation.

In the above example,

$$
m=\frac{\mathrm{AgCl}}{\mathrm{KCl}}=\frac{\mathrm{I} 43.34}{74.56}=\mathrm{I} .9223, n=\frac{\mathrm{AgCl}}{\mathrm{CsCl}}=\frac{\mathrm{I} 43.34}{\mathrm{I} 68.27}=0.8513,
$$

and

$$
m-n=1.0710 \text {. }
$$

Substituting these values in the general equation, we obtain,

$$
x=0.9337 p-0.7949 a .
$$

Consequently, in order to determine the weight of potassium chloride in the mixed sample it is only necessary to multiply the values of $a$ and $p$ by 0.7949 and 0.9337 , respectively, and subtract the first product from the second; i.e.,

$$
x=0.9337 \times 0.4889-0.3895 \times 0.7949=0.1470 \mathrm{~g} . \mathrm{KCl} \text {, }
$$

and $y=0.3895-0.1470=0.2425 \mathrm{~g} . \mathrm{CsCl}$.

The same analysis might be performed by weighing the mixed chlorides in a platinum crucible, then changing them to sulphates (by treatment with $\mathrm{H}_{2} \mathrm{SO}_{4}$, etc.), and again weighing. In this case also,

$$
\begin{aligned}
x & =\frac{\mathrm{I}}{m-n} \cdot p-\frac{n}{m-n} \cdot a \\
& =\mathrm{I} 0.66 p-\mathrm{II} .45 a .
\end{aligned}
$$

In the first case, the coefficients are relatively small, and consequently good results might be expected, since the experimental errors made in the determination of $a$ and $p$ are multiplied by only 0.7949 and 0.9337 , respectively. In the latter case, however, the coefficients are very large, and the unavoidable analytical errors would have to be multiplied enormously in the calculation; the latter method is therefore worthless.

Although some indirect methods may appear simple and attractive on paper, they frequently lead to impossible values in practice; so that extreme caution should be exercised regarding the use of an indirect method. In general, if accurate and reliable results are desired, indirect methods of analysis should be avoided. 


\section{PROBLEMS}

\section{GRAVIMETRIC ANALYSIS}

r. If the determination of a substance by a certain method permits of an error of $0.1 \%$ in the weighing of the sample, how accurately must a 200 mg. sample be weighed? A $5 \mathrm{~g}$. sample?

2. Calculate the chemical factors for the following: $\mathrm{KCl}$ from $\mathrm{K}_{2} \mathrm{PtCl}_{6}$; $\mathrm{K}_{2} \mathrm{O}$ from $\mathrm{KClO}_{4} ; \mathrm{MgO}$ from $\mathrm{Mg}_{2} \mathrm{P}_{2} \mathrm{O}_{7} ; \mathrm{Mn}$ from $\mathrm{Mn}_{2} \mathrm{P}_{2} \mathrm{O}_{7} ; \mathrm{MnO}_{2}$ from $\mathrm{Mn}_{3} \mathrm{O}_{4}$.

3. What weight of $\mathrm{Mn}_{3} \mathrm{O}_{4}$ is equivalent to $0.5785 \mathrm{~g}$. of $\mathrm{Mn}_{2} \mathrm{P}_{2} \mathrm{O}_{7}$ ? To 0.4327 g. of $\mathrm{MnSO}_{4}$ ?

4. A portion of phosphorus pentoxıde weighing $0.2018 \mathrm{~g}$. yields $0.3132 \mathrm{~g}$. of $\mathrm{Mg}_{2} \mathrm{P}_{2} \mathrm{O}_{7}$. Calculate the percentage of $\mathrm{P}_{2} \mathrm{O}_{5}$ in the sample.

5. A $0.4988 \mathrm{~g}$. sample of a salt, upon distillation with sodium hydroxide solution evolves ammonia, which is converted into $\left(\mathrm{NH}_{4}\right)_{2} \mathrm{PtCl}_{6}$; and this upon ignition yields $0.3258 \mathrm{~g}$. of platinum. Calculate directly from the weight of platinum the percentage of $\mathrm{NH}_{3}$ in the sample.

6. A quantity of the silver salt of an organic acid weighing $0.4072 \mathrm{~g}$. yields upon ignition $0.2632 \mathrm{~g}$. of metallic silver. Calculate the percentage of silver in the salt.

7. What weight of a silver nitrate solution known to contain $2.31 \%$ of $\mathrm{Ag}$ will be required to precipitate the chlorine from $25.0 \mathrm{cc}$. of a solution containing $12.5 \mathrm{~g}$. of $\mathrm{BaCl}_{2}, 2 \mathrm{H}_{2} \mathrm{O}$ in one liter?

8. If $25.0 \mathrm{cc}$. of sodium chloride solution yield $0.1434 \mathrm{~g}$. of silver chloride, what is the strength of the solution in grams of the salt per liter? In mols per liter?

9. How many cubic centimeters of hydrochloric acid of sp. gr. 1.050, containing $10.17 \%$ of $\mathrm{HCl}$, will it take to precipitate the silver from a solution containing $0.8430 \mathrm{~g}$. of silver sulphate?

I0. How many cubic centimeters of hydrochloric acid of sp. gr. 1.040, containing $8.16 \%$ of $\mathrm{HCl}$, will be required to dissolve one gram of calcium carbonate?

II. What weight of $\mathrm{Mn}_{2} \mathrm{P}_{2} \mathrm{O}_{7}$ is it possible to prepare from $50.0 \mathrm{cc}$. of a permanganate solution which contains 4.500 g. of $\mathrm{KMnO}_{4}$ per liter?

12. How many cubic centimeters of a solution of sp. gr. I.ri6, containing $10.06 \%$ of $\mathrm{NaOH}$, will it take to neutralize a solution containing $5.00 \mathrm{~g}$. of $\mathrm{NaHSO}_{4}$ ? $5.00 \mathrm{~g}$. of $\mathrm{KHSO}_{4}$ ?

13. A sample of impure potassium sulphide weighing $0.4320 \mathrm{~g}$. is treated with hydrochloric acid, and by means of ammoniacal hydrogen peroxide solution the hydrogen sulphide evolved is converted into ammonium sul- 
phate. This yields $0.8034 \mathrm{~g}$. of $\mathrm{BaSO}_{4}$. Calculate the percentage of $\mathrm{K}_{2} \mathrm{~S}$ in the sample.

14. A sample of stibnite weighing $1.078 \mathrm{~g}$., upon being analyzed by the method indicated in Problem ${ }^{3} 3$, yields 0.6750 g. of $\mathrm{BaSO}_{4}$. Assuming the sulphur to be present wholly as $\mathrm{Sb}_{2} \mathrm{~S}_{3}$, calculate the percentage of the latter in the mineral.

15. How many cubic centimeters of aqueous ammonia of sp. gr. 0.96, containing $9.91 \%$ of $\mathrm{NH}_{3}$, will be required to precipitate the aluminum in $0.8674 \mathrm{~g}$. of $\mathrm{KAl}\left(\mathrm{SO}_{4}\right)_{2} .12 \mathrm{H}_{2} \mathrm{O}$ ? How many cubic centimeters of 6-normal ammonia?

16. What volume of the ammonia water first referred to in Problem 15 will it take to neutralize $10.0 \mathrm{cc}$. of hydrochloric acid of sp. gr. I.I2, containing $23.8 \mathrm{I} \%$ of $\mathrm{HCl}$ ? To neutralize $10.0 \mathrm{cc}$. of 6-normal hydrochloric acid?

I7. If I5.0 cc. of a solution of barium chloride yield, upon evaporation with hydrochloric acid and gentle ignition, I.563 g. of the anhydrous salt, what is the strength of the solution in mols per liter? In equivalents per liter?

18. A solution contains $2.25 \%$ by weight of $\mathrm{BaCl}_{2}$. What volume of a solution containing 5.000 g. of $\mathrm{Ag}_{2} \mathrm{SO}_{4}$ per liter will be required to precipitate the chlorine in $5.000 \mathrm{~g}$. of the barium chloride solution? What will be the weight of the precipitate formed?

I9. A sample of pyritic mineral weighing $0.2637 \mathrm{~g}$. yields upon analysis 0.7993 g. of $\mathrm{BaSO}_{4}$. Upon the assumption that the sulphur is wholly present as $\mathrm{FeS}_{2}$, calculate the percentage of the latter in the mineral.

20. What volume of bromine water containing $3.0 \%$ by weight of bromine will be required to oxidize the iron in $\mathrm{I} .75 \mathrm{~g}$. of $\mathrm{FeSO}_{4} \cdot{ }_{7} \mathrm{H}_{2} \mathrm{O}$ ?

2r. What volume of aqueous ammonia (sp. gr. 0.96 , containing $9.91 \%$ of $\mathrm{NH}_{3}$ ) will be required, after oxidation of the iron with hydrogen peroxide, to precipitate the metal from a solution made by dissolving $0.750 \mathrm{~g}$. of $\mathrm{Fe}\left(\mathrm{NH}_{4} \mathrm{SO}_{4}\right)_{2} .6 \mathrm{H}_{2} \mathrm{O}$ in water acidified with $\mathrm{I} 2.0 \mathrm{cc}$. of hydrochloric acid (sp. gr. I.I2, containing $23.8 \%$ of $\mathrm{HCl}$ )?

22. A mixed sample of $\mathrm{CaO}, \mathrm{Ca}(\mathrm{OH})_{2}$, and $\mathrm{CaCO}_{3}$ weighing $0.5896 \mathrm{~g}$. is evaporated with excess sulphuric acid, and gently ignited; the residue weighs $0.865 \mathrm{I} \mathrm{g}$. What volume of 6-normal hydrochloric acid will be required to convert $5.00 \mathrm{~g}$. of the sample into calcium chloride?

23. A sample of pyrolusite weighing $0.5124 \mathrm{~g}$. is heated in the presence of dilute sulphuric acid with an excess of oxalic acid, and the gas evolved is absorbed in a weighed bulb containing potassium hydroxide. The gain in weight of the bulb is found to be $0.4789 \mathrm{~g}$. Calculate the percentage of $\mathrm{MnO}_{2}$ in the pyrolusite. 
24. The ignited mixture of ferric and aluminum oxides from $1.497 \mathrm{~g}$. of a mineral weighs $0.4196 \mathrm{~g}$; ; after a second ignition, in a current of hydrogen, the product weighs 0.33 I I g., the ferric oxide being reduced to metallic iron. Calculate the percentage of $\mathrm{Fe}_{2} \mathrm{O}_{3}$ and of $\mathrm{Al}_{2} \mathrm{O}_{3}$ in the mineral.

25. What volume of 0.5 -normal ammonium oxalate solution will be required to precipitate, as $\mathrm{CaC}_{2} \mathrm{O}_{4} \cdot \mathrm{H}_{2} \mathrm{O}$, the calcium from one gram of apatite, $\left[\mathrm{Ca}_{3}\left(\mathrm{PO}_{4}\right)_{2}\right]_{3}$. $\mathrm{CaF}_{2}$ ?

26. What volume of a solution containing $66 \mathrm{~g}$. of $\left(\mathrm{NH}_{4}\right)_{2} \mathrm{HPO}_{4}$ per liter will be required to precipitate, as $\mathrm{ZnNH}_{4} \mathrm{PO}_{4}$, the zinc from $0.9786 \mathrm{~g}$. of a brass which contains $30.15 \%$ of zinc? What is the normality of this solution as a precipitant for zinc?

27. How many grams per liter of $\left(\mathrm{NH}_{4}\right)_{2} \mathrm{Cr}_{2} \mathrm{O}_{7}$ must a solution contain in order that, by reduction of the chromium (with $\mathrm{HCl}$ and $\mathrm{SO}_{2}$ ), precipitation with ammonia, and ignition of the precipitate in a current of hydrogen, a 25.0 cc. portion shall yield 0.1267 g. of $\mathrm{Cr}_{2} \mathrm{O}_{3}$ ?

28. What volume of 6-normal sulphuric acid will be required to replace the nitric acid in the salts obtained upon evaporating to dryness the solution in nitric acid of a five cent coin weighing $4.960 \mathrm{~g}$., the composition of the coin being $75.00 \% \mathrm{Cu}$ and $25.00 \% \mathrm{Ni}$ ?

29. A sample of silicate mineral weighing $\mathrm{x} .0245 \mathrm{~g}$. yields $0.2602 \mathrm{~g}$. of potassium and sodium chlorides; and the mixed chlorides yield $0.4304 \mathrm{~g}$. of $\mathrm{K}_{2} \mathrm{PtCl}_{6}$. Calculate the percentage of $\mathrm{Na}_{2} \mathrm{O}$ in the sample.

30. A solution of chloroplatinic acid contains $0.050 \mathrm{~g}$. of Pt per cubic centimeter. What is the minimum volume with which $0.2602 \mathrm{~g}$. of mixed sodium and potassium chlorides must be evaporated in order to insure the complete conversion of the alkali metals into chloroplatinates, no matter in what proportions the two chlorides may exist in the mixture?

3r. A sample of phosphate rock contains $0.87 \%$ of moisture and $9 \mathbf{r} .92 \%$ of calcium phosphate. Calculate the percentage of $\mathrm{Ca}_{3}\left(\mathrm{PO}_{4}\right)_{2}$ which is present on the dry basis.

32. If $2.497 \mathrm{~g}$. of a fertilizer containing $4.45 \%$ of moisture yields $0.3150 \mathrm{~g}$. of $\mathrm{Mg}_{2} \mathrm{P}_{2} \mathrm{O}_{7}$, what is the percentage of $\mathrm{P}_{2} \mathrm{O}_{5}$ on the dry basis?

33. Upon treatment with sulphuric acid, $\mathrm{r} .430 \mathrm{~g}$. of a salt yields $0.595^{2} \mathrm{~g}$. of $\mathrm{Na}_{2} \mathrm{SO}_{4}$ and Ior.5 cc. of $\mathrm{CO}_{2}$, measured moist at $17^{\circ} \mathrm{C}$. and $757 \mathrm{~mm}$. Calculate the percentages of $\mathrm{Na}_{2} \mathrm{O}$ and $\mathrm{CO}_{2}$ in the salt. (Tension of aqueous vapor at $17^{\circ}=\mathrm{I} 4.45 \mathrm{~mm}$.)

34. A sample of silicate mineral weighing $0.8 \mathrm{r} 96 \mathrm{~g}$. yields $0.2082 \mathrm{~g}$. of potassium and sodium chlorides; and the mixed chlorides yield $0.1963 \mathrm{~g}$. of potassium perchlorate. Calculate the percentage of $\mathrm{K}_{2} \mathrm{O}$ and of $\mathrm{Na}_{2} \mathrm{O}$ in the mineral. 
35. What weight of water is present in one liter of air which is $50 \%$ saturated with moisture at $\mathrm{r} 7^{\circ}$ and $748 \mathrm{~mm}$.? (See problem 33.)

36. I.3250 g. of pure $\mathrm{Na}_{2} \mathrm{CO}_{3}$ is dissolved in water and the solution made up accurately to $250.0 \mathrm{cc}$. A portion is carefully transferred without loss to a platinum dish by means of a pipette supposed to deliver $50.00 \mathrm{cc}$. of liquid. After evaporation with hydrochloric acid, and ignition, the sodium chloride residue is found to weigh $0.2927 \mathrm{~g}$. What volume of this solution does the pipette actually deliver?

37. $0.7500 \mathrm{~g}$. of a substance containing chlorine and bromine yields $0.5000 \mathrm{~g}$. of $\mathrm{Ag}(\mathrm{Cl}, \mathrm{Br})$. This mixture is heated in a current of chlorine, which converts the bromide of silver into the chloride, and the loss in weight due to this change is found to be $0.0683 \mathrm{~g}$. Calculate the percentages of chlorine and bromine in the sample.

38. From the following data, calculate the percentages of chlorine, bromine, and iodine in a mixture of alkali halides: Weight of sample, 0.1 500 g.; weight of precipitate obtained upon distilling the solution with nitrous acid and converting the iodine into silver iodide, $0.1056 \mathrm{~g}$.; weight of silver chloride and bromide from the residual solution, $0.1784 \mathrm{~g}$.; weight of silver chloride obtained upon warming the latter mixture in a current of chlorine gas, $0.1623 \mathrm{~g}$.

39. A $0.5000 \mathrm{~g}$. sample of baking powder, known to contain only $\mathrm{NaHCO}_{3}$ and $\mathrm{KHC}_{4} \mathrm{H}_{4} \mathrm{O}_{6}$, in equivalent proportions, and starch, yields upon treatment with water $30.5 \mathrm{cc}$. of dry $\mathrm{CO}_{2}\left(0^{\circ}\right.$ and $760 \mathrm{~mm}$.). Calculate the percentage of each salt in the material.

40. A salt containing barium, chlorine, and water of hydration gave upon analysis the following data: Weight of sample, r.0000 g.; weight after heating (water driven off), $0.8522 \mathrm{~g}$.; weight of silver chloride obtained, I.r 735 g.; weight of barium sulphate obtained, $0.9594 \mathrm{~g}$. Calculate : (a) the percentage of each constituent; $(b)$ the formula of the compound.

4I. If two $1.0000 \mathrm{~g}$. samples of a substance containing $10.00 \%$ of $\mathrm{MgO}$ are weighed out, and the precipitate of $\mathrm{MgNH}_{4} \mathrm{PO}_{4} .6 \mathrm{H}_{2} \mathrm{O}$ is in one case contaminated with $0.0250 \mathrm{~g}$. of $\mathrm{Mg}_{3}\left(\mathrm{PO}_{4}\right)_{2}$, and in the other case with $0.0250 \mathrm{~g}$. of $\mathrm{Mg}\left[\left(\mathrm{NH}_{4}\right)_{2} \mathrm{PO}_{4}\right]_{2}$, what percentages of $\mathrm{MgO}$ will be found if the calculations are based upon the assumption that the ignited precipitate in each case consists entirely of $\mathrm{Mg}_{2} \mathrm{P}_{2} \mathrm{O}_{7}$ ?

42. The carbonates of calcium, strontium, and barium obtained from a ro-liter sample of mineral water are converted into the anhydrous nitrates, and the calcium nitrate is extracted with absolute alcohol-ether mixture. The residue is dissolved in water, the barium separated from the strontium, as $\mathrm{BaCrO}_{4}$, and the strontium precipitated from the filtrate with sulphuric acid and alcohol. There are finally obtained $0.8507 \mathrm{~g}$. of $\mathrm{CaO}, 0.1324 \mathrm{~g}$. 
of $\mathrm{SrSO}_{4}$, and $0.1072 \mathrm{~g}$. of $\mathrm{BaCrO}_{4}$; calculate the content of the water in milligrams per liter (i.e. parts per million) of $\mathrm{Ca}$, of $\mathrm{Sr}$, and of $\mathrm{Ba}$.

43. How many cubic centimeters of sulphuric acid of sp. gr. r.840, containing $95.6 \%$ of $\mathrm{H}_{2} \mathrm{SO}_{4}$, must be added to I liter of sulphuric acid of sp. gr. I. 560 , containing $65.1 \%$ of $\mathrm{H}_{2} \mathrm{SO}_{4}$, to yield a solution containing $75.0 \%$ of $\mathrm{H}_{2} \mathrm{SO}_{4}$ ?

44. A fuming sulphuric acid contains $25.5 \%$ of non-hydrated $\mathrm{SO}_{3}$. How many grams of $98.2 \% \mathrm{H}_{2} \mathrm{SO}_{4}$ must be added to $100 \mathrm{~g}$. of the fuming acid to give a product containing $100 \%$ of $\mathrm{H}_{2} \mathrm{SO}_{4}$ ?

45. A limestone contains $90.0 \%$ of $\mathrm{CaCO}_{3}, 3.50 \%$ of $\mathrm{MgCO}_{3}, 3.00 \%$ of $\mathrm{CaSO}_{4} \cdot 2 \mathrm{H}_{2} \mathrm{O}, \mathrm{x} .25 \%$ of $\mathrm{FeCO}_{3}$, and $2.25 \%$ of anhydrous siliceous material. What numerical difference would you expect to find between the loss on ignition and the true percentage of $\mathrm{CO}_{2}$ ?

46. An ore contains $28.15 \%$ of nickel, and $0.5000 \mathrm{~g}$. samples are taken for analysis. In one sample the element is determined by electrolysis, as metallic nickel, while in a second sample it is determined by means of dimethylglyoxime, as $\mathrm{Ni}\left(\mathrm{C}_{4} \mathrm{H}_{7} \mathrm{~N}_{2} \mathrm{O}_{2}\right)_{2}$. If the algebraic sum of the errors involved in each determination were equivalent to a negative error of r.7 mg. of the substance finally weighed, how much greater would the percentage error be in the first determination than in the second?

47. An electric current is passed simultaneously through a series of three electrolytic cells which contain water acidified with sulphuric acid, an ammoniacal solution of nickel sulphate, and molten silver chloride. What is deposited upon the cathode in each of the other cells, and how many grams, in the time in which one liter of hydrogen, measured moist at $17^{\circ}$ and $746 \mathrm{~mm}$., is liberated from the water? (Tension of aqueous vapor at $\mathrm{I} 7^{\circ}=\mathrm{I} 4.45 \mathrm{~mm}$.)

48. From the following data, calculate the percentages of nickel and cobalt in the steel: Weight of sample, I.I24 g.; weight of nickel and cobalt obtained upon electrolysis, o.1246 g.; weight of nickel dimethylglyoximine, $\mathrm{Ni}\left(\mathrm{C}_{4} \mathrm{H}_{7} \mathrm{~N}_{2} \mathrm{O}_{2}\right)_{2}$, obtained from the electrolytic deposit, $0.4382 \mathrm{~g}$.

49. A mass of platinum weighs $\mathrm{r} 2 . \mathrm{I} 45 \mathrm{~g}$. in air, $\mathrm{rr} .580 \mathrm{~g}$. in water, and Ir.rr5 g. in sulphuric acid. What is the specific gravity of the platinum? Of the sulphuric acid?

50. A quantity of pure metallic silver weighing $1.0788 \mathrm{~g}$. is dissolved in nitric acid and the solution made up to the mark in a measuring flask graduated to contain $100.0 \mathrm{cc}$. Three portions are carefully transferred without loss to three separate beakers by means of a pipette known to deliver $25.00 \mathrm{cc}$. If the solution remaining in the flask, together with the liquid finally washed from the pipette, yields on analysis $0.3560 \mathrm{~g}$. of $\mathrm{AgCl}$, what volume of liquid does the flask actually contain? 


\section{VOLUMETRIC ANALYSIS}

5r. If $25.00 \mathrm{cc}$. of hydrochloric acid yield $0.1435 \mathrm{~g}$. of $\mathrm{AgCl}$, what is the normality of the solution?

52 . If a $25.00 \mathrm{cc}$. portion of acid requires $21.50 \mathrm{cc}$. of $0.526 \mathrm{~N}$ alkali for neutralization, what is the normality of the acid? Supposing the acid to be $\mathrm{HCl}$, what weight of silver chloride will ro.00 cc. of it yield with silver nitrate?

53. If a $2.453 \mathrm{~g}$. sample of pure anhydrous sodium carbonate requires 45.72 cc. of an acid for neutralization, and if $4 \mathrm{I} .90 \mathrm{cc}$. of the acid requires $44.35 \mathrm{cc}$. of an alkali, what is the normality factor of each solution?

54. A sample of pure calcite, $\mathrm{CaCO}_{3}$, weighing 2.I50 g. is dissolved in $50.00 \mathrm{cc}$. of an acid, and the excess of acid is neutralized with $29 . \mathrm{r} 2 \mathrm{cc}$. of an alkali of which $28.40 \mathrm{cc}$. require 7. Io cc. of the acid for neutralization. To what volume must one liter of the acid be diluted in order to make it exactly normal?

55. How many cubic centimeters of $0.526 \mathrm{~N}$ acid will it take to neutralize the ammonia set free upon distilling $\mathrm{I} .0378 \mathrm{~g}$. of $\mathrm{MgNH}_{4} \mathrm{PO}_{4} \cdot 6 \mathrm{H}_{2} \mathrm{O}$ with an excess of caustic alkali?

56. If $15.25 \mathrm{cc}$. of alkali will neutralize $20.00 \mathrm{cc}$. of a solution containing $6.000 \mathrm{~g}$. of $\mathrm{KH}_{3}\left(\mathrm{C}_{2} \mathrm{O}_{4}\right)_{2} \cdot 2 \mathrm{H}_{2} \mathrm{O}$ in $250.0 \mathrm{cc}$., what is the normality factor of the alkali?

57. In the analysis of a feeding stuff, a Kjeldahl determination is carried out with a sample weighing $1.500 \mathrm{~g}$. The ammonia is received in $25.00 \mathrm{cc}$. of $0.500 \mathrm{~N}$ acid, and the excess of acid is found to require r2.50 cc. of a standard alkali, of which 2 I.20 cc. will neutralize I $8.00 \mathrm{cc}$. of the acid. Calculate the percentage of nitrogen in the sample.

58. A sample of soda weighing $25.00 \mathrm{~g}$. is dissolved in water and made up to $250.0 \mathrm{cc}$., and one fifth of this solution is taken for titration. What must be the normality of the standard acid (assuming the alkalinity to be due wholly to $\mathrm{Na}_{2} \mathrm{CO}_{3}$ ) in order that twice the number of cubic centimeters of acid used shall indicate the percentage of $\mathrm{Na}_{2} \mathrm{CO}_{3}$ in the sample?

59. What weight of argol (crude cream of tartar) must be taken for titration in order that each cubic centimeter of $0.2000 \mathrm{~N}$ alkali used shall represent $2.00 \%$ of $\mathrm{KHC}_{4} \mathrm{H}_{4} \mathrm{O}_{6}$ ?

6o. A sample of caustic soda weighing $4.000 \mathrm{~g}$. is dissolved in water and made up to one liter. A I00.0 cc. portion of this solution requires for neutralization $47.50^{\circ} \mathrm{cc}$. of $0.2000 \mathrm{~N}$ acid. A second roo.0 cc. portion, after treatment with barium chloride in slight excess, is diluted to $200.0 \mathrm{cc}$. 
and allowed to settle, and $50.0 \mathrm{cc}$. of the clear solution require Ir.50 cc. of the acid. Calculate the percentages of $\mathrm{NaOH}$ and $\mathrm{Na}_{2} \mathrm{CO}_{3}$ in the sample. (Neglect the volume occupied by the solid precipitate.)

6i. A sample of:Solvay soda weighing $3.750 \mathrm{~g}$. is dissolved in water and made up to one liter. A roo.o cc. portion of this solution, titrated in the cold with $0.1000 \mathrm{~N}$ acid, with the use of phenolphthalein, is found to require $29.95 \mathrm{cc}$. of the acid; the burette is then refilled and the titration completed at the boiling temperature of the solution, $35.15 \mathrm{cc}$. more of the acid being required. Calculate the percentages of $\mathrm{Na}_{2} \mathrm{CO}_{3}$ and $\mathrm{NaHCO}_{3}$ in the sample. (Under suitable experimental conditions, phenolphthalein becomes colorless in the cold as soon as the carbonate has been wholly converted into bicarbonate.)

62. A sample of sirupy phosphoric acid weighing $5.767 \mathrm{~g}$. is dissolved in water and made up to one liter. A roo:0 cc. portion of the solution is treated with sodium acetate and silver nitrate in excess, whereby the phosphate is quantitatively precipitated as $\mathrm{Ag}_{3} \mathrm{PO}_{4}$. Phenolphthalein is added to the filtrate and washings, and the solution titrated with $0.500 \mathrm{~N}$ alkali, of which $27.25 \mathrm{cc}$. are required. Calculate the percentage of $\mathrm{H}_{3} \mathrm{PO}_{4}$ in the original sample.

63. A sample of Chili saltpeter weighing $1.025 \mathrm{~g}$. is treated in sodium hydroxide solution with pulverized-Devarda's alloy $(50 \% \mathrm{Cu}, 45 \% \mathrm{Al}$, $5 \% \mathrm{Zn}$ ); which reduces the nitrogen to ammonia; the ammonia is distilled into $25.00 \mathrm{cc}$. of $0.463 \mathrm{~N}$ acid, and the excess of acid requires 5.or cc. of $0.212 N$ alkali for neutralization. Assuming that nitrogen was wholly present as $\mathrm{NaNO}_{3}$, calculate the percentage of the latter in the sample.

64. A sample of strontium nitrate weighing $10.53 \mathrm{~g}$. is dissolved in water and made up to one liter. One tenth of this solution is distilled, in the presence of alkali, with an excess of titanous chloride, which reduces the nitrate to ammonia $\left(\mathrm{KNO}_{3}+8 \mathrm{Ti}(\mathrm{OH})_{3}+6 \mathrm{H}_{2} \mathrm{O}=\mathrm{KOH}+8 \mathrm{Ti}(\mathrm{OH})_{4}+\right.$ $\mathrm{NH}_{3}$ ). The ammonia is received in $25.00 \mathrm{cc}$. of $0.500 \mathrm{~N}$ acid, and the excess of acid requires I0.16 cc. of $0.250 \mathrm{~N}$ alkali. Assuming that the nitrogen was wholly present as $\operatorname{Sr}\left(\mathrm{NO}_{3}\right)_{2}$, calculate the percentage of the latter in the sample.

65. How many grams of $\mathrm{KH}_{3}\left(\mathrm{C}_{2} \mathrm{O}_{4}\right)_{2} \cdot{ }_{2} \mathrm{H}_{2} \mathrm{O}$ will it take to prepare one liter of a $0.500 \mathrm{~N}$ solution, to be used as a standard acid? How many to prepare one liter of a $0.1000 N$ solution, to be used as a reducing agent in connection with potassium permanganate?

66. How many grams of $\mathrm{K}_{2} \mathrm{Cr}_{2} \mathrm{O}_{7}$ per liter will be required to prepare a solution of such strength that each cubic centimeter shall indicate $2.00 \%$ of iron, when a sample weighing $0.2792 \mathrm{~g}$. is used for analysis? What is the normality factor of this solution? 
67. From the following data, calculate the percentage of iron in the ore: Weight of sample, $0.2 \mathrm{I} 86 \mathrm{~g}$.; the reduced iron solution requires for oxidation $25.14 \mathrm{cc}$. of $0.0996 \mathrm{~N}$ permanganate solution.

68. What is the maximum weight of an ore containing $70.00 \%$ of iron which can be taken for analysis without having to refill a 30-cc. burette, if the permanganate solution is $0.1025 N$ ?

69. A calcium oxalate precipitate, obtained from $0.8432 \mathrm{~g}$. of a rock, is decomposed with dilute sulphuric acid and made up to $250.0 \mathrm{cc}$; of this a $50.0 \mathrm{cc}$. portion is titrated with $0.10 \mathrm{I} 2 \mathrm{~N}$ permanganate solution. If $27.35 \mathrm{cc}$. of the latter are required, what percentage of $\mathrm{CaO}$ does the rock contain?

70. I I ,56 cc. of nitric acid of sp. gr. I,19 are diluted to $250.0 \mathrm{cc}$; 20.00 cc. of this solution are found to require $12.92 \mathrm{cc}$. of 0.4 ro $N$ alkali. Calculate the percentage of $\mathrm{HNO}_{3}$ in the acid of sp. gr. I.I 9 .

$7 \mathrm{r}$. The $\mathrm{Sb}_{2} \mathrm{~S}_{3}$ precipitate obtained from the solution of an ore is dissolved in sodium sulphide solution, and this is evaporated and fumed with an excess of sulphuric acid. The residue is then dissolved in dilute hydrochloric acid, and the antimony oxidized from the trivalent to the penta valent condition by means of a standard solution of permanganate. The sample of ore weighs $0.2749 \mathrm{~g}$. and $24.17 \mathrm{cc}$. of $0.1025 \mathrm{~N}$ permanganate solution are used in the titration; what is the percentage of antimony?

72. What is the normality factor of an acid, of which $25.37 \mathrm{cc}$. are equivalent to $\mathrm{r}_{2} 63 \mathrm{~g}$. of $\mathrm{KNO}_{3}$ when the nitrogen of the latter is reduced in alkaline solution to ammonia and this is distilled off and received in the acid solution?

73. From the following data, what is the percentage of $\mathrm{MnO}_{2}$ in the ore? Weight of sample, $0.2000 \mathrm{~g}$; after heating this in the presence of sulphuric acid with $50.00 \mathrm{cc}$. of $0.1000 \mathrm{~N}$ oxalic acid, the excess of oxalic acid requires $8.50 \mathrm{cc}$. of $1.0000 \mathrm{~N}$ permanganate solution.

74. From the following data, what is the percentage of $\mathrm{MnO}_{2}$ in the ore? Weight of sample, $0.2500 \mathrm{~g}$. ; this is boiled with hydrochloric acid and the distillate received in an excess of potassium iodide solution, the liberated iodine requiring $25.5 \mathrm{I} \mathrm{cc}$. of $0.2000 \mathrm{~N}$ thiosulphate solution.

75. A standard solution of permanganate will oxidize $5.783 \mathrm{mg}$. of ferrous iron per cubic centimeter; to what volume must one liter of the solution be diluted in order to obtain an exactly tenth-normal solution?

76. A sample of mineral substance weighing $1.000 \mathrm{~g}$. is taken for analysis. In the determination of the iron, the ferric solution is completely reduced by means of sulphurous acid, and the excess of the latter removed by the passage of carbon dioxide through the boiling solution; the iron then requires $28.17 \mathrm{cc}$. of $0.1000 \mathrm{~N}$ permanganate solution. Calculate the percentage of iron in the substance. 
77. What weight of $\mathrm{Fe}\left(\mathrm{NH}_{4} \mathrm{SO}_{4}\right)_{2} .6 \mathrm{H}_{2} \mathrm{O}$ will reduce 50.0 cc. of a permanganate solution, of which $10.00 \mathrm{cc}$. will liberate from an acidified solution of potassium iodide a quantity of iodine sufficient to react with I $5.25 \mathrm{cc}$. of $0.1025 \mathrm{~N}$ thiosulphate solution?

78. The calcium oxalate precipitate obtained from $0.2500 \mathrm{~g}$. of calcite is dissolved in an excess of sulphuric acid, and the hot solution titrated with a solution of permanganate of which each cubic centimeter represents $0.00735 \mathrm{~g}$. of $\mathrm{Na}_{2} \mathrm{C}_{2} \mathrm{O}_{4}$. If $45.57 \mathrm{cc}$. of the permanganate solution are required, what percentage of calcium does the mineral contain?

79. A standard solution of permanganate will oxidize $0.00730 \mathrm{~g}$. of ferrous iron per cubic centimeter; what is the value of the same solution in terms of (a) $\mathrm{H}_{2} \mathrm{C}_{2} \mathrm{O}_{4} \cdot 2 \mathrm{H}_{2} \mathrm{O}$; (b) $\mathrm{NaNO}_{2} ;(c) \mathrm{H}_{2} \mathrm{O}_{2} ;($ d $) \mathrm{K}_{4} \mathrm{Fe}(\mathrm{CN})_{6} \cdot 3 \mathrm{H}_{2} \mathrm{O}$; (e) $\mathrm{Mn}$ ?

80 . If $0.1340 \mathrm{~g}$. of sodium oxalate require $19.23 \mathrm{cc}$. of a permanganate solution, how many milligrams of ferrous iron will each cubic centimeter of the permanganate solution indicate?

$8 \mathrm{I}$. From the following data, calculate the percentage of manganese in the ore: Weight of sample, $0.5027 \mathrm{~g}$; volume of permanganate solution required to oxidize the manganese, $36.60 \mathrm{cc}$; value of the permanganate solution for use with oxalic acid, $0.0997 \mathrm{~N}$.

82. 2.400 liters of dry air (at $\circ^{\circ}, 760 \mathrm{~mm}$.) and $50.00 \mathrm{cc}$. of $0.0100 \mathrm{~N}$ barium hydroxide solution are shaken together, and the excess of alkali is found to require $35.06 \mathrm{cc}$. of $0.0100 \mathrm{~N}$ acid. What volume of carbon dioxide is contained in 10,000 volumes of the dry air?

83. If a permanganate solution is equivalent to $5.84 \mathrm{mg}$. of iron per cubic centimeter, what is the value of the solution in terms of $\mathrm{K}_{4} \mathrm{Fe}(\mathrm{CN})_{6} \cdot 3 \mathrm{H}_{2} \mathrm{O}$ ? In terms of $\mathrm{N}_{2} \mathrm{O}_{3}$ ?

84. What weight of iodine per cubic centimeter will be liberated by a permanganate solution from an excess of hydriodic acid, if the permanganate solution has an iron value of $4.98 \mathrm{mg}$. per cubic centimeter?

85. A piece of iron wire weighing 0.1408 g., and containing $99.84 \%$ of iron, is converted into ferrous chloride and titrated according to the Zimmermann-Reinhardt method; it requires $25.15 \mathrm{cc}$. of a permanganate solution. What weight of ore must be taken for analysis by the same method, in order that each cubic centimeter of the permanganate solution shall indicate $2.50 \%$ of iron?

86. $25.00 \mathrm{cc}$. of a certain acid are found to require $23.67 \mathrm{cc}$. of an alkali. If $28.15 \mathrm{cc}$. of the acid are used to dissolve $0.5260 \mathrm{~g}$. of pure calcium carbonate, and 6.6I cc. of the alkali are required to neutralize the resulting solution, what is the normality factor of the acid? What is that of the alkali?

87. The calcium oxalate from $0.5165 \mathrm{~g}$. of a mineral requires, after 
decomposition with sulphuric acid, $43.56 \mathrm{cc}$. of a permanganate solution having a sodium oxalate value of $6.70 \mathrm{mg}$. per cubic centimeter. What is the percentage of $\mathrm{CaO}$ in the mineral?

88. In the standardization of a dichromate solution, a sample of pure iron weighing $0.2000 \mathrm{~g}$. is converted into $200 \mathrm{cc}$. of ferrous chloride solution, and titrated. In the subsequent analysis of an ore with the dichromate solution, an equal quantity of ferrous iron is present, but the solution to be titrated has a volume of $600 \mathrm{cc}$. If the indicator used permits the recognition of one part by weight of ferrous iron in 200,000 of solution, what error results from the fact that the two titrations are made at different volumes? (Assume that the dichromate solution was found to be $0.1000 \mathrm{~N}$, and that the solutions have the specific gravity of water.)

89. A sample of crystalline ammonium acetate weighing $2.02 \mathrm{I} \mathrm{g}$. is dissolved in water and made up to $200.0 \mathrm{cc}$. One half of this solution is distilled with an excess of lime, and the distillate received in $25.00 \mathrm{cc}$. of $0.500 \mathrm{~N}$ acid; the second half is distilled with phosphoric acid in excess, and the distillate received in $45.00 \mathrm{cc}$. of $0.500 \mathrm{~N}$ alkali. In the first case, with methyl orange, the excess of acid requires $10.20 \mathrm{cc}$. of the standard alkali, and in the second case, with phenolphthalein, the excess of alkali requires $15.52 \mathrm{cc}$. of the standard acid. Calculate the formula of the salt.

90 . The aqueous solution of $0.136 \mathrm{r}$ g. of a mixture containing only sodium chloride and bromide is treated, in the presence of nitric acid, with $25.00 \mathrm{cc}$. of $0.1000 \mathrm{~N} \mathrm{AgNO}_{3}$ solution, and the precipitate is filtered off and washed. The filtrate and washings require $9.70 \mathrm{cc}$. of $0.1000 \mathrm{~N}$ thiocyanate solution. Calculate the percentages of sodium chloride and bromide in the sample.

$\checkmark$ 9I. A mixture containing soluble chlorides and iodides weighs $0.4500 \mathrm{~g}$. This is treated, in the presence of nitric acid, with $35.00 \mathrm{cc}$. of $0.1000 \mathrm{~N}$ silver nitrate solution, and the precipitate is found to weigh $0.5000 \mathrm{~g}$. The excess of silver nitrate in the filtrate and washings requires II.ro cc. of $0.0500 \mathrm{~N}$ thiocyanate solution. Calculate the percentages of chlorine and iodine in the original mixture.

92. A $20.00 \mathrm{cc}$. portion of a prussic acid solution is made slightly alkaline with sodium hydroxide, and a very little potassium iodide is added; the solution is then titrated with $0.1000 N$ silver nitrate, of which 48.73 cc. are required to produce a faint permanent turbidity of silver iodide. Calculate the percentage of $\mathrm{HCN}$ in the original solution, assuming the specific gravity of the latter to be that of water.

93. A mixture containing potassium cyanide and chloride, and weighing $0.2037 \mathrm{~g}$., is dissolved in water and titrated with $0.1000 \mathrm{~N}$ silver nitrate solution, of which $14.4 \mathrm{I}$ cc. are required to produce a faint permanent turbidity. $25.00 \mathrm{cc}$. more of the silver nitrate solution are added, the, 
solution is slightly acidified with nitric acid, and the filtrate and washings from the precipitate require for titration 8.9I cc. of $0.1000 \mathrm{~N}$ thiocyanate solution. Calculate the percentages of $\mathrm{KCN}$ and $\mathrm{KCl}$ in the sample.

94. A solution of potassium permanganate is equivalent to $5.00 \mathrm{mg}$. of iron per cubic centimeter. To $40.0 \mathrm{cc}$. of this solution, acidified with an excess of very dilute sulphuric acid, an excess of potassium iodide is added and the liberated iodine is titrated with a solution of sodium thiosulphate, of which $34.85 \mathrm{cc}$. are required. Calculate the normality factor of the thiosulphate solution.

95. A sample of bleaching powder weighing $7.092 \mathrm{~g}$. is triturated with water and made up to one liter. A $50.0 \mathrm{cc}$. portion of this suspension, when titrated with $0.1000 N$ arsenious acid, with potassium iodide-starch paper as an outside indicator, is found to require $26.15 \mathrm{cc}$. of the standard solution. What is the percentage of available chlorine in the bleaching powder?

96. A sample of soda weighing $4.973 \mathrm{~g}$. is dissolved in water and made up to one liter. A I00.0 cc. portion of this solution, carefully titrated in the cold with phenolphthalein as an indicator, requires $48.90 \mathrm{cc}$. of $0.0998 \mathrm{~N}$ acid. A second portion of $100.0 \mathrm{cc}$. is titrated with $0.499 \mathrm{~N}$ acid, of which I3.8I cc. are required with methyl orange as an indicator. Calculate the percentages of $\mathrm{Na}_{2} \mathrm{CO}_{3}$ and $\mathrm{NaOH}$ in the sample. (Cf. Problem 6r.)

97. If $43.60 \mathrm{cc}$. of a thiosulphate solution require $40.15 \mathrm{cc}$. of an iodine solution, and if $0.2 \mathrm{II} 8 \mathrm{~g}$. of $\mathrm{As}_{2} \mathrm{O}_{3}$ require $42.40 \mathrm{cc}$. of the iodine solution, what is the normality factor of each solution?

98. A sample of titaniferous ore weighing $0.3805 \mathrm{~g}$. is fused with a mixture of $\mathrm{K}_{2} \mathrm{~S}_{2} \mathrm{O}_{7}$ and $\mathrm{KF}$, the melt dissolved in $\mathrm{HCl}$, and the iron and titanium reduced with zinc in an atmosphere of hydrogen. The solution is then titrated in an atmosphere of carbon dioxide, in the presence of $\mathbf{I}$ g. of KSCN as the indicator, with $0.1000 \mathrm{~N}$ ferric chloride solution, of which I9.34 cc. are required. Calculate the percentage of $\mathrm{TiO}_{2}$ in the ore. $\left(\mathrm{TiCl}_{3}+\mathrm{FeCl}_{3}\right.$ $=\mathrm{TiCl}_{4}+\mathrm{FeCl}_{2}$.)

99. In $50.0 \mathrm{cc}$. of a solution, containing both ferrous and ferric sulphates, the ferrous iron is titrated in the presence of sulphuric acid with $0.1000 \mathrm{~N}$ permanganate solution, after which the oxidized solution is titrated for total iron with $0.0997 N$ titanous chloride solution, with potassium thiocyanate as the indicator. If in the first titration $27.17 \mathrm{cc}$. of the permanganate solution are used, and in the second titration $46.98 \mathrm{cc}$. of the titanous chloride solution, what is the content of the original solution in grams per liter of ferrous and of ferric iron?

100. The ammonium phosphomolybdate precipitate obtained from $2.000 \mathrm{~g}$. of steel is dissolved in dilute aqueous ammonia, the solution acidified 
with sulphuric acid, and the molybdenum reduced to the trivalent condition by passing the acid solution through a Jones reductor, - the reduced solution being caused to enter the receiving vessel under the surface of a solution of ferric sulphate $\left(2 \mathrm{MoO}_{3}+6 \mathrm{H}=\mathrm{Mo}_{2} \mathrm{O}_{3}+3 \mathrm{H}_{2} \mathrm{O}\right.$; and $\mathrm{Mo}_{2} \mathrm{O}_{3}+3 \mathrm{Fe}_{2} \mathrm{O}_{3}=2 \mathrm{MoO}_{3}+6 \mathrm{FeO}$ ). The resulting solution is at once titrated with a standard solution of permanganate, of which $18.75 \mathrm{cc}$. are required. If the permanganate solution has an iron value of $0.00540 \mathrm{~g}$. per cubic centimeter, what is the percentage of phosphorus in the steel? 



\section{APPENDIX}

\section{THE PREPARATION OF THE REAGENTS}

MANY of the reagents which are used in quantitative analysis are made up for one purpose only; directions for the preparation of these are given in this book under the individual determinations. Certain reagents, however, are used in many operations at approximately fixed concentrations, and it is especially these which are included in this section.

It is of great advantage to have such stock reagents conform to some definite system of concentration, the most convenient system being one based upon the equivalent or normal weights employed in volumetric analysis. Of course, however, the concentrations of such solutions need not be so exactly fixed as for use in accurate volumetric work.

With this system, equal volumes of the solutions bear fixed relations to one another, it is easy to calculate the volume of a reagent required for a specific purpose, and the addition of an unnecessary excess may readily be avoided. Thus, if we dissolve I g. of calcium carbonate in ro cc. of 6-normal hydrochloric acid, and wish to neutralize the liquid with ammonia, we see at once that $\left(10-\frac{\mathrm{I}}{\frac{\mathrm{CaCO}_{3} \times 6}{2000}}\right)=\left(10-\frac{\mathrm{I}}{\frac{\mathrm{I00} \times 6}{2000}}\right)=6.7 \mathrm{cc}$. will be about the

right amount. Again, if we fuse $0.15 \mathrm{~g}$. of chromite with $4.0 \mathrm{~g}$. of $\mathrm{Na}_{2} \mathrm{O}_{2}$, extract the fusion with water, and wish to almost (but not quite) neutralize the aqueous extract, we see that $\frac{4}{\frac{\mathrm{Na}_{2} \mathrm{O}_{2} \times 6}{2000}}=\frac{4.0}{\frac{78 \times 6}{2000}}=$ I 7.I cc. of 6 -normal acid would neutralize 
the liquid; consequently we use ${ }^{5} 5^{-16} \mathrm{cc}$. of the acid, and test the resulting mixture to make sure of its alkalinity. As will be realized, this effects a saving in time, labor, and material; and also it leads to more accurate and reliable work.

Graduated cylinders and measuring pipettes are useful for delivering specific volumes of such reagents.

\section{ACIDS}

Acetic, 6-normal: Mix 350 cc. of glacial acetic acid with $650 \mathrm{cc}$. of water.

Hydrochloric, I2-normal: Use the C. P. acid of commerce of sp. gr., I.I9.

Hydrochloric, 6-normal: Mix I2-normal acid with an equal volume of water. The specific gravity of this acid is about I.IO.

Nitric, I6-normal: Use the C. P. acid of commerce of sp. gr., I.42.

Nitric, 6-normal: Mix $380 \mathrm{cc}$. of the r6-normal acid with $650 \mathrm{cc}$. of water. The specific gravity of this acid is about I.195.

Sulphuric, 36-normal: Use the C. P. acid of commerce of sp. gr., r.84.

Sulphuric, 6-normal: Pour $200 \mathrm{cc}$. of the 36-normal acid into 1045 cc. of water. The specific gravity of this acid is I.I8.

\section{BASES}

Ammonium hydroxide, I5-normal: Use the C. P. ammonia water of commerce of sp. gr., 0.90 .

Ammonium hydroxide, 6-normal: Mix $400 \mathrm{cc}$. of the 15normal solution with $600 \mathrm{cc}$. of water. The specific gravity of this solution is about 0.958 .

Sodium hydroxide, 6-normal: Dissolve $250 \mathrm{~g}$. of stick sodium hydroxide in water and dilute to one liter. 


\section{SALTS}

Ammonium carbonate: Dissolve $250 \mathrm{~g}$. of freshly powdered ammonium carbonate in one liter of 6-normal ammonium hydroxide, and filter if there is a residue.

Ammonium molybdate: ${ }^{1}$ Dissolve $100 \mathrm{~g}$. of $\mathrm{MoO}_{3}$ in $80 \mathrm{cc}$. of ammonia (sp. gr., 0.90 ) with the addition of $400 \mathrm{cc}$. of water; with cooling and constant stirring, allow the clear solution to run slowly into a mixture of $400 \mathrm{cc}$. of nitric acid (sp. gr., r.42) with $600 \mathrm{cc}$. of water, add $0.05 \mathrm{~g}$. of microcosmic salt, $\mathrm{NaNH}_{4} \mathrm{HPO}_{4} \cdot{ }_{4} \mathrm{H}_{2} \mathrm{O}$, and keep the mixture in a warm place for several days, or until a portion heated to $40^{\circ}$ deposits no yellow precipitate. Decant from any sediment, and preserve in glassstoppered bottles. This solution contains $68 \mathrm{~g}$. of $\mathrm{MoO}_{3}$ per liter. Ammonium oxalate, 0.5 -normal: Dissolve $35.5 \mathrm{~g}$. of $\left(\mathrm{NH}_{4}\right)_{2} \mathrm{C}_{2} \mathrm{O}_{4} \cdot \mathrm{H}_{2} \mathrm{O}$ in 1000 cc. of water.

Barium chloride, I-normal: Dissolve 122 g. of $\mathrm{BaCl}_{2} \cdot 2 \mathrm{H}_{2} \mathrm{O}$ in rooo cc. of water.

Magnesia mixture, 0.5-normal as a precipitant for phosphoric or arsenic acid: Dissolve $5 \mathrm{I} \mathrm{g}$. of $\mathrm{MgCl}_{2} .6 \mathrm{H}_{2} \mathrm{O}$ and $\mathrm{I} 30 \mathrm{~g}$. of $\mathrm{NH}_{4} \mathrm{Cl}$ in water, add I2I cc. of ammonia (sp. gr., o.9o), and dilute to one liter.

Mercuric chloride, o.2-normal for oxidizing stannous chloride: Dissolve $54 \mathrm{~g}$. of $\mathrm{HgCl}_{2}$ in $1000 \mathrm{cc}$. of hot water.

Silver nitrate, 0.2-normal: Dissolve $34 \mathrm{~g}$. of $\mathrm{AgNO}_{3}$ in $1000 \mathrm{cc}$. of water.

Sodium phosphate, 0.5-normal as a precipitant for magnesium: Dissolve 90 g. of $\mathrm{Na}_{2} \mathrm{HPO}_{4}, \mathrm{I}_{2} \mathrm{H}_{2} \mathrm{O}$ (or $52 \mathrm{~g}$. of $\mathrm{NaNH}_{4} \mathrm{HPO}_{4} \cdot \mathrm{H}_{2} \mathrm{O}$ ) in 1000 cc. of water.

${ }^{1}$ Recovery of the Molybdic Acid. To the liquid molybdate residues, acidified if necessary with nitric acid, add sodium phosphate solution in excess. Collect the yellow precipitate, wash it with water containing sodium sulphate, and then dry it in the air. Dissolve $\mathrm{I} \mathrm{Kg}$. of the dried precipitate in ammonia, add a strong solution of $60 \mathrm{~g}$. of $\mathrm{NH}_{4} \mathrm{Cl}$ and $\mathrm{I} 20 \mathrm{~g}$. of $\mathrm{MgCl}_{2}, 6 \mathrm{H}_{2} \mathrm{O}$ in water, allow to stand for 6 hours, and filter off the precipitate. To the filtrate, decolorized if necessary with a little $\mathrm{H}_{2} \mathrm{O}_{2}$, add $\mathrm{HCl}$ just to acid reaction, to precipitate the $\mathrm{MoO}_{3}$. Collect this precipitate, wash with water, and dry at $110^{\circ}$. 
Stannous chloride, I-normal as a reducing agent: Dissolve II 3 g. of $\mathrm{SnCl}_{2}, 2 \mathrm{H}_{2} \mathrm{O}$ in 150 cc. of I2-normal hydrochloric acid, with the gradual addition of water, finally diluting to one liter. Keep in bottles containing granulated tin.

\section{Sulphuric Acid-Dichromate Cleaning Solution}

With stirring, cautiously pour $200 \mathrm{cc}$. of sulphuric acid (sp. gr., r.84) into 150 cc. of cold water, and saturate the hot solution, without further heating, with powdered sodium (or potassium) dichromate.

When cleaning measuring vessels with this liquid, they should be filled with the cold solution and allowed to stand overnight, or longer.

\section{Analytical Samples for the Use of Students}

The analyzed samples indicated in the text for the use of beginners in quantitative analysis may in some cases be obtained in the market. Otherwise, they may be prepared by mixing together the component materials in the proportions decided upon. This mixing is best accomplished by long continued grinding in a ball mill, the material being finally passed through a fine-meshed sieve, and bottled. These samples should be carefully analyzed by members of the quantitative staff, so that the student's work may be judged according to its accuracy. Most of the mixtures indicated can be kept from year to year without change.

It is desirable to have in each case a continuous series of at least ten samples, varying in content from sample to sample by about $0.4-0.5 \%$. 


\section{APPARATUS IN THE STUDENT'S DESK ${ }^{1}$}

\section{Quantitative Chemical Laboratories}

\section{Above in the drawers}

I Brush, camel's hair.

I Burette, g. s., $30 \mathrm{cc}$.

I Burette, 30 cc., for pinchcock.

4 Crucibles, porcelain, 0 .

2 Crucibles, porcelain, Gooch, extra disc.

2 Cylinders, graduated, $50 \mathrm{cc}$. and ro cc.

I File.

I Forceps, steel.

2 Funnels, diam. 25 mm., stem $40 \mathrm{~mm}$.

2 Glasses, watch, $140 \mathrm{~mm}$.

2 Glasses, watch, $70 \mathrm{~mm}$.

2 Glasses, watch, $50 \mathrm{~mm}$.

I Vial litmus paper, blue.

I Vial litmus paper, red.

2 Boxes matches, safety.

I Pinchcock.

I Pipette, 25 cc.

I Pipette, Io cc.

2 Policemen, rubber tip.

3 Rods, glass, $200 \mathrm{~mm}$.

6 Test tubes.

I Thermometer, $100^{\circ} \mathrm{C}$.

I Tongs, brass, nickel plated.

I Tube, connecting, 3-way.

2 Tubes, rubber, for Gooch crucibles.

3 Tubes, weighing, with corks.

I Tube, rubber, pressure, length $300 \mathrm{~mm}$.

I Tube, rubber, small, length $300 \mathrm{~mm}$.

Tubing, soft glass, $900 \mathrm{~mm}$.

\section{Below in the cupboard}

12 Beakers, 2 nests, I-6, with window pane.

2 Bottles, g. S., 2500 cc.

I Bottle, g. s., $250 \mathrm{cc}$., for cleaning solution.

I Bottle, g. S., I25 cc., for silver nitrate.

I Bottle, weighing.

I Burette holder, Lincoln's

2 Burners, adjustable.

2 Burner tubes, rubber.

2 Casseroles, porcelain, $500 \mathrm{cc}$.

I Desiccator for 4 crucibles.

2 Flasks, Erlenmeyer, $700 \mathrm{cc}$.

2 Flasks, Erlenmeyer, $500 \mathrm{cc}$.

2 Flasks, Erlenmeyer, $250 \mathrm{cc}$.

2 Flasks, Erlenmeyer, I $50 \mathrm{cc}$.

2 Flasks, filter, $500 \mathrm{cc}$.

2 Flasks, Florence, $500 \mathrm{cc}$.

2 Flasks, Florence, $250 \mathrm{cc}$.

2 Flasks, Florence, $50 \mathrm{cc}$., for indicators.

I Flask, volumetric, I000 cc.

I Flask, volumetric, $500 \mathrm{cc}$.

2 Flasks, volumetric, $250 \mathrm{cc}$.

I Flask, volumetric, I0o cc.

4 Funnels, diam. $70 \mathrm{~mm}$., stem $200 \mathrm{~mm}$.

2 Funnels, for Gooch crucibles.

I Sponge.

I Stand, filter, wooden.

2 Stands, iron, I ring each.

2 Triangles, pipe stem, new form.

2 Tripods, iron.

2 Wire gauzes.

1 The articles listed above represent the apparatus with which it is desirable to provide each student at the outset; the list can of course be modified in many particulars without jeopardizing the success of the work. Any additional apparatus which may be required can be obtained as needed from the storeroom. 


\begin{tabular}{|c|c|c|c|c|c|c|c|c|c|c|c|c|c|c|}
\hline$N$ & 0 & 1 & 2 & 3 & 4 & 5 & 6 & 7 & 8 & 9 & 12 & & 456 & 789 \\
\hline 1 & & & & & 70 & & 53 & 294 & 34 & 374 & 8 & & 125 & 37 \\
\hline & & & & & & & & & & & & & & \\
\hline & & & & & & & & & & & & & & \\
\hline & & & 206 & & 1271 & & & 367 & 399 & 1430 & & & & \\
\hline 14 & & & & & 84 & 14 & & & 703 & 1732 & & & & \\
\hline & & & & & & & & & & & & & & \\
\hline & & & 95 & 122 & 148 & 175 & 201 & 2227 & 2253 & 2279 & 3 & & & \\
\hline 17 & 304 & 330 & 55 & 380 & 405 & 130 & & 2480 & 2504 & 2529 & & 7 & & \\
\hline & & & & & 648 & & & & & & & & & \\
\hline & & 10 & 33 & 356 & 878 & 900 & 2923 & 945 & 2967 & 2989 & 2 & & & \\
\hline 20 & 010 & 32 & 54 & 3075 & 096 & 118 & 3139 & 3160 & 3181 & 3201 & 24 & 6 & & \\
\hline & 202 & & & & & & & & & & & & & \\
\hline & & & & & & & & 560 & 3579 & & & & & \\
\hline & 3617 & 36 & 55 & 3674 & 3692 & 711 & 3729 & 3747 & 3766 & 3784 & 2 & & & \\
\hline 2 & 302 & 320 & 38 & 3856 & 3874 & 3892 & 3909 & 27 & 3945 & 962 & & & & \\
\hline & & & & & & & & 99 & 116 & 133 & & & & \\
\hline & 4150 & 166 & 83 & 4200 & 4216 & 232 & 4249 & 4265 & 4281 & 4298 & 23 & & 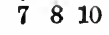 & \\
\hline 27 & 314 & 4330 & 346 & 362 & 378 & 393 & 4409 & 4425 & 4440 & 4456 & & 5 & & \\
\hline & & & & & & & & & 594 & & & & & \\
\hline 2 & 624 & & & 669 & 83 & 698 & & 4 & & 4757 & 13 & 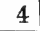 & 67 & \\
\hline 30 & 4771 & 4786 & 4800 & 4814 & |4829 & 4843 & 4857 & 4871 & 4886 & 4900 & 13 & 4 & $\begin{array}{ll}6 & 7\end{array}$ & \\
\hline 3 & 14 & & & 55 & 69 & 983 & & 011 & 024 & 038 & & & & \\
\hline & & & & & & & & 45 & 5159 & & & & & \\
\hline & 185 & 98 & 5211 & 5224 & 5237 & 250 & 63 & 276 & 5289 & 5302 & & 4 & 7 & \\
\hline 3 & 315 & 328 & 340 & 353 & 366 & 378 & 5391 & 5403 & 5416 & 5428 & & & & \\
\hline & & & & & & & & & 639 & 51 & & & & \\
\hline & & & & & & & & 647 & 5658 & 5670 & 1 & & 6 & \\
\hline 37 & 682 & & 5705 & 5717 & 29 & 5740 & & 763 & 5775 & & & & & \\
\hline & & & & & & & & & & & & & & \\
\hline & 11 & & 33 & & & & & & & & & & & \\
\hline 40 & 021 & 31 & 6042 & & 64 & 6075 & 85 & 096 & 07 & 117 & 12 & 3 & 45 & 8910 \\
\hline 41 & 108 & & 6 & & & & & & 212 & 6222 & 1 & 3 & 5 & \\
\hline & & & & & & & & & & & & & & \\
\hline 4 & & & & & & & & & & 425 & 12 & & 5 & 789 \\
\hline 44 & 6435 & 14 & & 6464 & & 6484 & 6493 & 6503 & 6513 & 6522 & 1 & 3 & 5 & \\
\hline & & & & & & & & & & & & & & $\pi$ \\
\hline & & & & & & & & & & & 1 & & 5 & 7 \\
\hline & 6721 & 6730 & 6739 & 6749 & 758 & 6767 & 6776 & 6785 & 6794 & 6803 & 1 & 3 & 45 & \\
\hline & & & & & & & & & & & & & & \\
\hline & 302 & 311 & & & 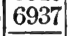 & & & 364 & 972 & 801 & 2 & & $4 \quad 4$ & 0 \\
\hline 50 & 6990 & 6998 & 7007 & 7016 & 7024 & 7033 & 7042 & 7050 & 7059 & 7067 & 12 & 3 & $\begin{array}{ll}34 \\
\end{array}$ & $\begin{array}{lll}678 \\
\end{array}$ \\
\hline & 07 & 70 & 709 & 7101 & 7110 & $\overline{7118}$ & 7126 & 7135 & & & & & & \\
\hline & & & & & & & & & & & & & & $\therefore$ \\
\hline & & & & 1206 & & 7284 & & 1000 & 7308 & & & 2 & 34 & $\begin{array}{lll}6 & 67\end{array}$ \\
\hline 54 & $0.02 \pi$ & 7332 & 7340 & 7348 & 7356 & 7364 & 7372 & 7380 & 7388 & $\underline{7396}$ & 12 & 2 & $\begin{array}{ll}3 & 4 \\
\end{array}$ & 667 \\
\hline & 0 & 1 & $\mathrm{~N}$ & 8 & 4 & & & & & 9 & 12 & 2 & $\begin{array}{lll}4 & 5 & 6\end{array}$ & 789 \\
\hline
\end{tabular}

The proportional parts are stated in full for every tenth at the right-hand side. Tho logarithm of any number of four significant figures can be read directly by add- 


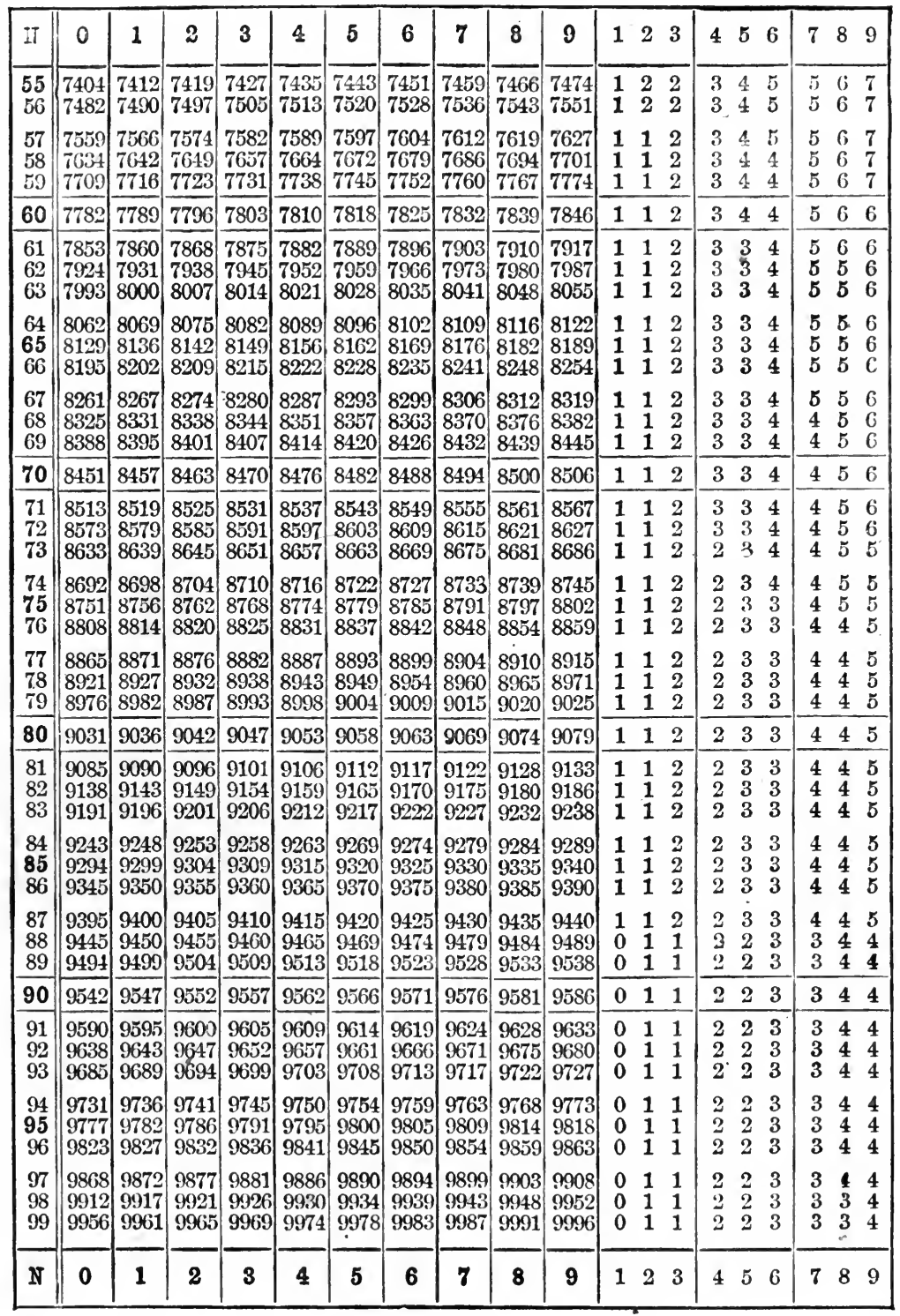

ing the proportional part corresponding to the fourth figure to the tabular number corresponding to the first three figures. There may be an error of 1 in the last place. 


\begin{tabular}{|c|c|c|c|c|c|c|c|c|c|c|c|c|c|c|c|c|c|c|c|}
\hline & 0 & 1 & 2 & 3 & 4 & 5 & 6 & 7 & 8 & 9 & & 2 & & & 5 & & 7 & 8 & \\
\hline .00 & 1000 & 1002 & 1005 & 1007 & 1009 & 1012 & 1014 & 1016 & 1019 & 1021 & 0 & 0 & 1 & 1 & 1 & 1 & 2 & 2 & 2 \\
\hline $\begin{array}{l}.01 \\
.02\end{array}$ & $\begin{array}{l}1023 \\
1047\end{array}$ & $\begin{array}{l}1026 \\
1050\end{array}$ & \begin{tabular}{|l|}
1028 \\
1052
\end{tabular} & $\begin{array}{l}1030 \\
1054\end{array}$ & $\begin{array}{l}1033 \\
1057\end{array}$ & $\begin{array}{l}1035 \\
1059\end{array}$ & $\begin{array}{l}1038 \\
1062\end{array}$ & $\begin{array}{l}1040 \\
1064\end{array}$ & $\begin{array}{l}1042 \\
1067\end{array}$ & $\begin{array}{l}1045 \\
1069\end{array}$ & $\begin{array}{l}0 \\
0\end{array}$ & $\begin{array}{l}0 \\
0\end{array}$ & & $\begin{array}{l}1 \\
1\end{array}$ & $\begin{array}{l}1 \\
1\end{array}$ & 1 & $\frac{2}{2}$ & & $\begin{array}{l}2 \\
2\end{array}$ \\
\hline .03 & 1072 & 1074 & 1076 & 1079 & 1081 & 1084 & 1086 & 1089 & 1091 & 1094 & 0 & 0 & 1 & & 1 & 1 & 2 & & 2 \\
\hline .04 & 1096 & 1099 & 1102 & 1104 & 1107 & 1109 & 1112 & 1114 & 1117 & 1119 & 0 & 1 & 1 & 1 & 1 & $?$ & 2 & $\Omega$ & 2 \\
\hline .05 & $\begin{array}{l}1122 \\
1148\end{array}$ & $\begin{array}{l}1125 \\
1151\end{array}$ & $\begin{array}{l}1127 \\
1153\end{array}$ & $\begin{array}{l}1130 \\
1156\end{array}$ & $\begin{array}{l}1132 \\
1159\end{array}$ & $\begin{array}{l}1135 \\
1161\end{array}$ & $\begin{array}{l}1138 \\
1164\end{array}$ & $\begin{array}{l}1140 \\
1167\end{array}$ & $\begin{array}{l}1143 \\
1169\end{array}$ & $\begin{array}{l}1146 \\
1172\end{array}$ & $\begin{array}{l}0 \\
0\end{array}$ & $\begin{array}{l}1 \\
1\end{array}$ & 1 & & $\begin{array}{l}1 \\
1\end{array}$ & 2 & & & $\begin{array}{l}2 \\
2\end{array}$ \\
\hline .07 & 1175 & 1178 & 1180 & 1183 & 1186 & 1189 & 1191 & 1194 & 1197 & 1199 & 0 & 1 & 1 & 1 & 1 & 2 & $?$ & 2 & 2 \\
\hline .08 & 1202 & 1205 & 1208 & 1211 & 1213 & 1216 & 1219 & 1222 & 1225 & 1227 & 0 & 1 & & & 1 & & & 2 & 0 \\
\hline .09 & 1230 & 1233 & 1236 & 1239 & 1242 & 1245 & 1247 & 1250 & 1253 & 1256 & 0 & 1 & 1 & & 1 & 2 & & 2 & 3 \\
\hline .10 & 1259 & 1262 & 1265 & 1268 & 1271 & 1274 & 1276 & 1279 & 1282 & 1285 & 0 & 1 & 1 & 1 & 1 & 2 & 2 & 2 & 3 \\
\hline .11 & 1288 & 1291 & 1294 & 1297 & 1300 & 1303 & 1306 & 1309 & 1312 & 1315 & 0 & 1 & 1 & 1 & 2 & 2 & 2 & 2 & 3 \\
\hline .12 & 318 & 1321 & 1324 & 1327 & 1330 & 1334 & 1337 & 1340 & 1343 & 1346 & 0 & 1 & & 1 & 2 & & & 2 & \\
\hline .13 & 1349 & 1352 & 1355 & 1358 & 1361 & 1365 & 1368 & 1371 & 1374 & 1377 & 0 & 1 & 1 & & 2 & 2 & & 3 & 3 \\
\hline .14 & 1380 & 1384 & 1387 & 1390 & 1393 & 1396 & 1400 & 1403 & 1406 & 1409 & 0 & 1 & 1 & 1 & 2 & 2 & & 3 & .3 \\
\hline .15 & 1413 & $\mid 1416$ & 1419 & 1422 & 1426 & & 1432 & 1435 & 1439 & 1442 & 0 & 1 & & 1 & 2 & & & 3 & 3 \\
\hline .16 & 1440 & 1449 & 1402 & 1400 & 1409 & 1402 & 1460 & 1469 & 1472 & 1476 & 0 & 1 & 1 & & 2 & 2 & & 3 & 3 \\
\hline .17 & 1479 & 1483 & 1486 & 1489 & 1493 & 1496 & 1500 & 1503 & 1507 & 1510 & 0 & 1 & 1 & 1 & 2 & 2 & & 3 & 3 \\
\hline .18 & 1014 & 1517 & 1521 & 1524 & 1528 & 1531 & 1535 & 1538 & 1542 & 1545 & 0 & 1 & & & 2 & & & 3 & 3 \\
\hline & & & & & & & & 1014 & & 1001 & 0 & 1 & 1 & & 2 & & & & 5 \\
\hline .20 & 1585 & 1589 & 1592 & 1596 & 1600 & 1603 & 1607 & 1611 & 1614 & 1618 & 0 & 1 & 1 & 1 & 2 & 2 & 3 & 3 & 3 \\
\hline .21 & 1622 & $\mid 1626$ & 1629 & 1633 & 1637 & 1641 & 1644 & 1648 & 1652 & 1656 & 0 & 1 & 1 & & 2 & 2 & & 3 & 3 \\
\hline .22 & 1660 & 1663 & 1667 & 1671 & 1675 & 1679 & 1683 & 1687 & 1690 & 1694 & 0 & 1 & & 2 & 2 & & & 3 & 3 \\
\hline .23 & 1698 & 1702 & 1706 & 1710 & 1714 & 1718 & 1722 & 1726 & 1730 & 1734 & 0 & 1 & 1 & 2 & 2 & 2 & & & 3 \\
\hline .24 & 1738 & $\mid 1742$ & 1746 & 1750 & 1754 & 1758 & 1762 & 1766 & 1770 & 1774 & 0 & 1 & 1 & 2 & 2 & 2 & 3 & 3 & 4 \\
\hline .25 & 1778 & 1782 & 1786 & 1791 & 1795 & 1799 & 1803 & 1807 & 1811 & 1816 & 0 & 1 & & 2 & 2 & & & 3 & 4 \\
\hline .26 & 1820 & 1824 & 1828 & 1832 & 1837 & 1841 & 1845 & 1849 & 1854 & 1858 & 0 & 1 & 1 & & 2 & 3 & 3 & 3 & 4 \\
\hline .27 & 1862 & 1866 & 1871 & 1875 & 1879 & 1884 & 1888 & 1892 & 1897 & 1901 & 0 & 1 & 1 & 2 & 2 & 3 & & & 4 \\
\hline .28 & 1905 & 1910 & 14 & 1919 & 1923 & 1928 & 1932 & 1936 & 1941 & 1945 & 0 & 1 & 1 & 2 & 2 & & & & 4 \\
\hline & 1900 & 1954 & 1909 & 1963 & 1968 & 1972 & 1977 & 1982 & 1986 & 1991 & 0 & 1 & 1 & 2 & 2 & 3 & 3 & 4 & 4 \\
\hline .30 & 1995 & 2000 & 2004 & 2009 & 2014 & 2018 & 2023 & 2028 & 2032 & 2037 & 0 & 1 & 1 & 2 & 2 & 3 & 3 & 4 & 4 \\
\hline .31 & 2042 & 2046 & 2051 & 2056 & 2061 & 2065 & 2070 & 2075 & 2080 & 2084 & 0 & 1 & 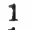 & 2 & 2 & 3 & & 4 & 4 \\
\hline .32 & 2089 & 2094 & 2099 & 2104 & 2109 & 2113 & 2118 & 2123 & 2128 & 2133 & 0 & 1 & & 2 & 2 & & & & 4 \\
\hline .33 & 2138 & 2143 & 2148 & 2153 & 2158 & 2163 & 2168 & 2173 & 2178 & 2183 & 0 & 1 & 1 & 2 & 2 & 3 & 3 & 4 & 4 \\
\hline .34 & 2188 & 2193 & 2198 & 2203 & 2208 & 2213 & 2218 & 2223 & 2228 & 2234 & 1 & 1 & 2 & 2 & 3 & 3 & & & 5 \\
\hline 35 & 2239 & 2244 & 2249 & 2254 & |2259 & 2265 & 2270 & 2275 & 2280 & 2286 & 1 & 1 & & & & & & & 5 \\
\hline .36 & 2291 & 2296 & 2301 & 2307 & 2312 & 2317 & 2323 & 2328 & 2333 & 2339 & 1 & 1 & 2 & 2 & 3 & 3 & 4 & \pm & 5 \\
\hline .37 & 2344 & 2350 & 2355 & 2360 & 2366 & 2371 & 2377 & 2382 & 2388 & 2393 & 1 & 1 & & 2 & 3 & & 4 & & 5 \\
\hline 38 & 2399 & 2404 & 2410 & 2415 & 2421 & 2427 & 2432 & 2438 & 2443 & 2448 & 1 & 1 & & 2 & 3 & $?$ & 4 & & 5 \\
\hline .39 & 2450 & 2460 & 2466 & 2472 & 2477 & 2483 & 2489 & 2495 & 2500 & 2506 & 1 & 1 & 2 & 2 & 3 & 3 & 4 & 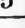 & 3 \\
\hline .40 & 2512 & 2518 & 2523 & 2529 & 2535 & 2541 & 2547 & 2553 & 2559 & 2564 & 1 & 1 & 2 & 2 & 3 & 4 & 4 & 5 & 5 \\
\hline .41 & 2570 & 2576 & 2582 & 2588 & 2594 & 2600 & 2606 & 2612 & 2618 & 2624 & & & & 2 & 3 & 4 & & 5 & 6 \\
\hline .4 & $\begin{array}{l}2630 \\
2692\end{array}$ & \begin{tabular}{|l|}
2636 \\
2698
\end{tabular} & $\begin{array}{l}2642 \\
2704\end{array}$ & $\begin{array}{l}2649 \\
2710\end{array}$ & $\begin{array}{l}2655 \\
2716\end{array}$ & $\begin{array}{l}2661 \\
2723\end{array}$ & $\begin{array}{l}2667 \\
2729\end{array}$ & $\begin{array}{l}2673 \\
273\end{array}$ & 2679 & $\begin{array}{l}2685 \\
2748\end{array}$ & $\begin{array}{l}1 \\
1\end{array}$ & $\begin{array}{l}1 \\
1\end{array}$ & & $\begin{array}{l}2 \\
2\end{array}$ & $\begin{array}{l}3 \\
3\end{array}$ & $\begin{array}{l}4 \\
4\end{array}$ & $\begin{array}{l}4 \\
4\end{array}$ & 5 & 6 \\
\hline & 2754 & 2761 & & & & 2786 & & 2799 & 2805 & 2812 & 1 & & & 3 & 3 & 4 & 4 & & 6 \\
\hline .40 & 2818 & 28 & 2831 & & & 2851 & 2358 & 2864 & 2871 & 2877 & 1 & & & & & & & & \\
\hline .46 & 2884 & 2891 & 2897 & 2904 & 2911 & 2917 & 2924 & 2931 & 2938 & 2944 & 1 & 1 & 2 & 3 & 3 & 4 & 5 & 5 & 6 \\
\hline .47 & 2951 & 2958 & 2965 & 2972 & 2979 & 2985 & 2992 & 2999 & 3006 & 3013 & 1 & 1 & 2 & 3 & 3 & 4 & 5 & & 6 \\
\hline .48 & 3020 & 3027 & 3034 & 3041 & 3048 & 3055 & 3062 & 3069 & 3076 & 3083 & 1 & 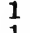 & & 3 & & & 5 & & \\
\hline .49 & 3090 & |3097| & & 3112 & 3119 & 3126 & 3133 & 3141 & 3148 & 3155 & 1 & & & & 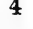 & & & & \\
\hline
\end{tabular}




\begin{tabular}{|c|c|c|c|c|c|c|c|c|c|c|c|c|c|c|c|}
\hline & 0 & 1 & 2 & 3 & 4 & 5 & 6 & 7 & 8 & 9 & 1.2 & & & 56 & $\begin{array}{lll}7 & 8 & 9\end{array}$ \\
\hline .50 & 3162 & 3170 & 3177 & 3184 & 3192 & 3199 & 3206 & 3214 & 3221 & 3228 & 11 & & & $4 \quad 4$ & $\begin{array}{lll}5 & 6 & 7\end{array}$ \\
\hline .51 & $\begin{array}{l}3236 \\
3311\end{array}$ & $\begin{array}{l}3243 \\
3319\end{array}$ & $\begin{array}{l}3251 \\
3327\end{array}$ & $\begin{array}{l}3258 \\
3334\end{array}$ & $\begin{array}{l}3266 \\
3342\end{array}$ & $\begin{array}{l}3273 \\
3350\end{array}$ & $\begin{array}{l}3281 \\
3357\end{array}$ & $\begin{array}{l}3289 \\
3365\end{array}$ & $\begin{array}{l}3296 \\
3373\end{array}$ & $\begin{array}{l}3304 \\
3381\end{array}$ & $\begin{array}{ll}1 & 1 \\
1 & 1\end{array}$ & 2 & $\begin{array}{l}3 \\
3\end{array}$ & $\begin{array}{ll}4 & 4 \\
4 & 5\end{array}$ & $\begin{array}{lll}5 & 6 & 7 \\
5 & 6 & 7\end{array}$ \\
\hline .53 & 3388 & 3396 & 3404 & 3412 & 3420 & 3428 & 3436 & 3443 & 3451 & 3459 & & 2 & & 45 & $\begin{array}{lll}6 & 67 & 7\end{array}$ \\
\hline .54 & 3467 & 3475 & 3483 & 3491 & 3499 & 3508 & 3516 & 3524 & 3532 & 3540 & & 2 & 3 & $4{ }^{\prime} 5^{\prime}$ & $\begin{array}{lll}6 & 6 & 7\end{array}$ \\
\hline .55 & 3548 & & 3565 & 3573 & 3581 & 3589 & 3597 & 3606 & 3614 & 3622 & & & & & 6 \\
\hline .56 & 3631 & 3639 & 3648 & 3656 & 3664 & 3673 & 3681 & 3690 & 3698 & 3707 & 12 & 2 & & 45 & 678 \\
\hline .57 & 3715 & 3724 & 3733 & 3741 & 3750 & 3758 & 3767 & 3776 & 3784 & 3793 & & 3 & & 5 & 78 \\
\hline .58 & 3802 & & 3819 & 3828 & 3837 & 3846 & & 3864 & 3873 & 3882 & & & & & $\begin{array}{ll}678 \\
\end{array}$ \\
\hline & & & & 0010 & & & & & 0000 & & & & & & \\
\hline .60 & 3981 & 3990 & 3999 & 4009 & 4018 & 4027 & 4036 & 4046 & 4055 & 4064 & 12 & 3 & 4 & $\begin{array}{l}56 \\
\end{array}$ & $\begin{array}{l}88 \\
\end{array}$ \\
\hline .61 & 4074 & 4083 & 4093 & 4102 & 4111 & 4121 & 4130 & 4140 & 4150 & 4159 & 12 & 3 & & 56 & 8 \\
\hline .62 & 4169 & 4178 & 4188 & 4198 & 4207 & 4217 & 4227 & 4236 & 4246 & 4256 & 12 & 3 & & & 9 \\
\hline .63 & 4266 & 4276 & 4285 & 4295 & 4305 & 4315 & 4325 & 4335 & 4345 & 4355 & 12 & 3 & & 56 & \\
\hline .64 & 4365 & 4375 & 4385 & 4395 & 4406 & 4416 & 4426 & 4436 & 4446 & 4457 & 2 & 3 & & 6 & 8 \\
\hline .65 & 4467 & 4477 & 4487 & 4498 & 4508 & 4519 & 4529 & 4539 & 4550 & 4560 & & ? & & 56 & $\begin{array}{ll}89 \\
9\end{array}$ \\
\hline .66 & 4571 & 4581 & 4592 & 4603 & 4613 & 4624 & 4634 & 4645 & 4656 & 4667 & 12 & 3 & & 56 & \\
\hline .67 & 4677 & 4688 & 4699 & 4710 & 4721 & 4732 & 4742 & 4753 & 4764 & 4775 & 12 & 3 & 4 & 7 & 910 \\
\hline .68 & 4786 & 4797 & 4808 & 4819 & 4831 & 4842 & & 4864 & 4875 & & 12 & 3 & & 6 & \\
\hline .69 & 4898 & 4909 & 4920 & 4932 & 4943 & 4955 & 4966 & 4977 & 4989 & 5000 & 12 & 3 & 5 & 7 & 910 \\
\hline .70 & 5012 & 5023 & 5035 & 5047 & 5058 & 5070 & 5082 & 5093 & 5105 & 5117 & 12 & 3 & 5 & $\begin{array}{ll}67 \\
\end{array}$ & $\begin{array}{l}8 \quad 910 \\
\end{array}$ \\
\hline .71 & 5129 & 5140 & 5152 & 5164 & 5176 & 5188 & 5200 & 5212 & 5224 & 5236 & & 4 & & 6 & 310 \\
\hline .72 & 5248 & 5260 & 5272 & 5284 & 5297 & 5309 & 5321 & 5333 & 5346 & 5358 & & & & & \\
\hline .73 & 5370 & 5383 & 5395 & 5408 & 5420 & 5433 & 5445 & 5458 & 5470 & 5483 & 13 & 4 & 5 & 67 & \\
\hline .74 & 5495 & 5508 & 5521 & 5534 & 5546 & 5559 & 5572 & 5585 & 5598 & 5610 & 1 & 4 & 5 & 8 & 1012 \\
\hline .75 & 5623 & 5636 & 5649 & 5662 & 5675 & 5689 & 5702 & 5715 & 5728 & 5741 & & & & & 12 \\
\hline .76 & 5754 & 5768 & 5781 & 5794 & 5808 & 5821 & 5834 & 5848 & 5861 & 5875 & & & 5 & 78 & 12 \\
\hline .77 & 5888 & 5902 & 5916 & 5929 & 5943 & 5957 & 5970 & 5984 & 5998 & 6012 & 13 & 4 & & 8 & 101112 \\
\hline .78 & 6026 & 6039 & 6053 & 6067 & 6081 & 6095 & 6109 & 6124 & 6138 & 6152 & & & & 8 & 1113 \\
\hline .79 & 6166 & 6180 & 6194 & 6209 & 6223 & 6237 & 6252 & 6266 & 6281 & 6295 & 13 & 4 & 6 & 7 & 13 \\
\hline .80 & 6310 & 6324 & 6339 & 6353 & 6368 & 6383 & 6397 & 6412 & 6427 & 6442 & 13 & 4 & 6 & 79 & 101213 \\
\hline .81 & 6457 & 6471 & 6486 & 6501 & $6 \check{16}$ & 6531 & 6546 & 6561 & 6577 & 6592 & 23 & 5 & & 8 & 111214 \\
\hline .82 & 307 & 6622 & 6637 & 6653 & 6668 & 6683 & 6699 & 6714 & 6730 & 6745 & & & & & \\
\hline .83 & 6761 & 6776 & 6792 & 6808 & 6823 & 6839 & 6855 & 6871 & 6887 & 6902 & 23 & 5 & & & 111314 \\
\hline .84 & 6918 & 6934 & 6950 & 6966 & 6982 & 6998 & 7015 & 7031 & 7047 & 7063 & $\begin{array}{ll}2 & 3 \\
0\end{array}$ & 5 & & & 111315 \\
\hline .85 & 7079 & 7096 & 12 & & & 61 & 178 & 7194 & 7211 & 7228 & & & & 810 & 315 \\
\hline .86 & 7244 & 7261 & & 7295 & 7311 & 7328 & 345 & 7362 & 7379 & 7396 & 23 & 5 & & 810 & 121415 \\
\hline .87 & 7413 & 7430 & 7447 & 7464 & 7482 & 7499 & 7516 & 7534 & 7551 & 7568 & 2 & 5 & & 910 & 121416 \\
\hline .88 & 7586 & 7603 & 7621 & 7638 & 7656 & 7674 & 7691 & 7709 & 7727 & 7745 & 24 & & & 911 & 416 \\
\hline .89 & 1862 & 1780 & 6798 & $\frac{7816}{7000}$ & 7834 & 7852 & 7870 & 7889 & 7907 & 7925 & 24 & 0 & 7 & & $\frac{131516}{1215.17}$ \\
\hline .90 & 7943 & 7962 & 7980 & 7998 & 8017 & 8035 & 8054 & 8072 & 8091 & 8110 & 24 & 6 & 7 & 911 & 131517 \\
\hline .91 & 8128 & 8147 & 8166 & 8185 & 8204 & & 8241 & 8260 & 0.79 & 8299 & & & & & \\
\hline & o & & & & 8395 & & & & 8472 & & & & & & \\
\hline .93 & 8511 & 8531 & 8551 & 8570 & 8590 & 8610 & & 8650 & 8670 & 8690 & 24 & & & & 141618 \\
\hline .94 & 8710 & 8730 & 8750 & 8770 & 8790 & 8810 & & 8851 & 8872 & 8892 & & & & & 141618 \\
\hline .95 & & & 8954 & 8974 & 8995 & 9016 & & 9057 & 9078 & 9099 & $x$ & & & & 719 \\
\hline .96 & 9120 & 9141 & 9162 & 9183 & 9204 & 9226 & 9247 & 9268 & 9290 & 9311 & 24 & & & & 151719 \\
\hline .97 & 9333 & 9354 & 9376 & 9397 & 9419 & 9441 & 9462 & 9484 & 9506 & 9528 & & & & & 151719 \\
\hline & 9550 & 9572 & 959 & & 963 & 9661 & & & 9727 & 9750 & & & & & \\
\hline .99 & 9772 & 9795 & 9817 & 9840 & 9863 & & & 9931 & 9954 & 9977 & & & & 114 & 161821 \\
\hline
\end{tabular}





\section{INDEX}

Accuracy . . . . . . . . 4

Acidimetry .. . . . . . I07, II8

Acids, degree of ionization of . . 25 determination of available hy-

- drogen-ion in . . . . . . II8 standard solutions of . . . . III titration of . . . . . . 108

Adsorption . . . . . . 23, 3I

Affinity constant . . . . . . 26

Afterflow, error from . . . . . 45

Alkalimeter, Mohr's . . . . 71, 72

Alkalimetry . . . . . 107, II6, I20

Alkali solutions, standard . . . III

Aluminum, determination of . . 63

Ammonium thiocyanate, standard solutions of . . . . . . 166

Ampere, definition of . . . . . 92

Analyzed chemicals . . . . . 3

Antilogarithms . . . . . . 212

Antimony, determination of in stibnite. . . . . . . 155

Apparatus, list of for quantitative work . . . . . . . 209

Arsenic, removal of from copper . ${ }_{1} 63$

Arsenious oxide, primary standard $\quad \mathrm{I}^{2} \mathrm{2}$

Asbestos, preparation of for filters 34 use of in filtration . . . . 34,35

Ashless filter papers . . . . . 3 I

Atomic weights, table of Back cover sheet

Baking powder, determination of carbon dioxide in

Balance, analytical 73,74 adjus analytical $\cdot \cdot \cdot \cdot 7$ adjustment of . . . . . 9 conditions to be fulfilled by . . 8 exercises with . . . . 53 location of . . . 8 relative length of arms of . . . 18 rest-point of . . . . . . . II sensitiveness of . . . . . 13 use and care of . . . . . . 9

Barium sulphate, properties of . 64,65
Bases, degree of ionization of . . 25 standard solutions of . . . . III titration of . . . . . . 108

Bumping . . . . . . $4^{2}$

Buoyancy, correction for . . . I9

Burettes . . . . . . . . 43

calibration of . . . . 48

cleaning of . . . . 45

reading of . . . . . . 45

Burning filter papers . . . . 39

Calcium, determination of in limestone . . . . . . 75,137 oxalate, properties of . . 78

Calibration of measuring vessels . 48 of weights . . . . . . 15

Carbon dioxide, determination of in limestone . . . . 7 $7 \mathbf{I}$ determination of in baking powders . . . . . . 73,74

Chemical equilibrium . . . 25

Chemical factors . . . . . 186

Chlorine, determination of $55,59,168$

Chrome iron ore, determination of chromium in . . . . 157

Chromic oxide, ignition of . . . 63

Chromium, determination of 63,157

Cleaning solution, preparation and use of . . . . . . 208

Colloidal precipitates . . . . 22

Common-ion effect . . . . . 27

Contamination of precipitates . . 23

Contat-Göckel valve . . . I38, I39

Copper, determination of . . 89, 162

Counterpoise, use of in weighing bulky objects . . . 2I, 74

Crucibles, materials of . . . 40

Current density . . . . . . 96

Current, production of for electroanalysis . . . . . 92

Decantation . . . . . . . 32

Decimal, number of places to report . . . . . . 6 
Deposition voltages of elements, table of . . . . . . . . 94

Desiccators . . . . . . . 39

Dichromate processes . . . . 125

solutions, standard . . . . . 125

Dichromate-sulphuric acid cleaning solution . . . . . 208

Digestion of precipitates . . . 22

Distilled water, testing of . . . 3

Double precipitation . . . . 24

Drainage, error from . . . . . 45

Drying ovens . . . . . 38

Electro-analysis . . . . . $\quad 89$

Electrode potentials, table of . . 94

Electrodes, material and form of . $\quad 97$

Electrolytes, influence of composition of upon electro-analysis . $\quad 96$

Electrolytic separations . . . 94

Electrolytic solution tension . $\quad 93$

End-point in titration, determination of

Equilibrium, chemical

Equilibrium constant . . . . 26

Evaporation of liquids . . . 42

Factor, chemical . . . . . . $\mathbf{I 8 6}$ normality . . . . . . 190

Faraday's laws . . . . . . 92

Ferric alum, indicator . . r66, r67

Ferric oxide, ignition of . . . 61, 63

Ferrous ammonium sulphate, standard solutions of . . . 125

Fertilizers, determination of nitrogen in . . . . 120,122

Filters, selection and use of . . . $3 \mathrm{I}$

Filtrates, testing of . . . . . 33

Filtration . . . . . . . . $3 \mathrm{I}$

Fine-grained precipitates, enlargement of the particles of . . 22

Flasks, volumetric . . . . . 44 calibration of . . . . . . 48

Flocculation of colloids . . . 22

Funnels, selection of . . . . . 32

Fusions, removal of from crucibles $\quad 85$

Glassware

Gooch filters, preparation and use of . . . . . . . . . 34, 35 sources of error with . . . . 36
Graduated cylinders . . . . 44

Gravimetric analysis . . . . I, 53

Halogens, determination of . . . 59

Hematite, determination of iron in . . . . . . . . . . 133

Hydrochloric acid, standard solutions of . . . . . . . . II2

Ignition of precipitates - . $\quad 38$

Indicators . . . 102, 107, 124 general theory of . . . . . 103 sensitiveness of in alkalimetry and acidimetry . . . 108, 109

Indirect methods of analysis . . I9I

Insoluble matter, determination of in limestone . . . . . .

International atomic weights, table of . . . Back cover sheet

Iodine standard solutions of $148, I_{5} I$

Iodometric processes . . . . $\mathrm{I}_{48}$

Ionization, degree of . . . . 25 repression of . . . . . . 27

Ions, complex . . . . . . 25 composition of the . . . . 25

Iron, determination of . 60, 129, 133 oxidation of ferrous to ferric

$$
6 \mathrm{r}, 62, \mathrm{I} 27, \mathrm{I} 33
$$

reduction of ferric to ferrous

$$
63,127,135
$$

Iron ores, decomposition of $129,134,135$

Iron wire, primary standard . 126

Jones reductor, assembly and use of . . . . . . . . $\mathbf{I 4}^{2}$

Kjeldahl, determination of protein nitrogen . . . : . . 120

Labels . . . . . . . . . 4

Laboratory Records . . . . . 5

Lead, determination of in an ore - 159

Limestone, analysis of 71, 75, 77, 137 determination of carbon dioxide in . . . . . . . . . $7 \mathrm{I}$

Limits of error in experimental work . . . . . . . . . 5

Liquids, evaporation of . . . 42 transference of . . . . . 42 volumetric measurement of . . 43 
Liter, Mohr's . . . . . . . 47 normal . . . . . . . . 47 true. . . . . . . . . 48

Logarithms . . . . . . . 2 ro

Magnesium, determination of in limestone . . . . . 75, 79

Magnesium ammonium phosphate, ignition of . . . .

Manganese, determination of in an ore . . . . . . . . . 144

Manganese ores, decomposition of 146

Methyl orange solution . . . . I ro

Molybdic acid, recovery of . . . 207

Neutral solution, definition of . . 107

Neutralization methods . . . IO 7

Nickel, electrolytic determination of $9 \mathrm{I}$

Nitrogen, Kjeldahl determination of . . . . . . . . . . $\mathrm{I} 20$

Normality factor, definition of $\quad$ r9o

Normal solutions . . . . . . ro

Normal System of Reagents, advantages of . . . . . 205

Ohm, definition of . . . . . 92

Ohm's law . . . . . . . 92

Ovens, drying . . . . . . . 38

Overvoltage . . . . . . . . . . 94

Oxidation and reduction methods 124

Oxidizing agents, standard solutions of . . . . . . . . 124

Parallax, error from . . . . 45

Permanganate processes . . . 130

Permanganate solutions, standard I3I

Phenolphthalein solution . . . I ro

Phosphoric anhydride, determination of

Phosphorus, determination of in steel .

Pipettes, transfer . . . . . . 44 calibration of . . . . . . . 48

Platinum ware, defects of modern $4 \mathrm{I}$ specifications for . . . . . $4 \mathrm{I}$ use and care of . . . . . 40, 4I, 89

Polarity of terminals, determination of . . . . . . $\quad . \quad 89$

Polarization . . . . . . . 94

Policeman, definition of . . . . 33

Potash, determination of . . . 68
Potassium bitartrate, primary standard

dichromate, standard solutions of . . . . . . . . . . 125 ferricyanide, indicator . . 125, 126 iodate, primary standard . . . $\mathbf{r}_{53}$ permanganate, standard solutions of .

thiocyanate, standard solutions of . . . . . . . . . 166

Precipitates, colloidal . . . 22

contamination of . . . . 23

digestion of . . . . . . .

drying of . . . . . . . . 38

enlargement of the particles of . 22

fine-grained . . . . . . 22

flocculation of . . . . . . 22

for use in gravimetric analysis . 21

ignition of . . . . . 38, 39

purification of . . . . . 24

washing of . . 21, 31, 33, 36

Precipitation . . . . . . 2 I

theory of . . . . . . . . 24

volumetric methods of . . 165

Problems . . . . . . . . 192

Pyrolusite, $\mathrm{MnO}_{2}$ value of . . ${ }_{13} 8$

Questions . . . . . . $\mathbf{r} 70$

Reactions of volumetric analysis . IOI

Reagents, analyzed . . . . 3

preparation of . . . . 205

quality of . . . . . . . 3

testing of . . . . . . . . 3

Records . . . . . . . . 5

Reducing agents, standard solutions of . . . . . . . . I 24

Reduction, methods of oxidation and . . . . . . . . I24

Reductor, Jones . . . . . . $\mathrm{I}_{42}$

Rest-point of balance, determination of . . . . . . . . I I

Reversible reactions . . . . 24

Salts, degree of ionization of . $\quad 25$

Samples, Preparation of, for Analysis . . . . . 50, 208

Saturated solution, definition of .29

Sensitiveness, of balance . . . $\quad$ r 3 of indicators, table of the . $\quad . \quad$ rog

Siderite, determination of iron in . 
Silica, determination of

Silicates, determination of silica in refractory . . . . . . $\quad 85$

Silicic acid, dehydration of . . 87

Silver, determination of . . . 59, I65

Silver, primary standard . . . 167

Silver chloride, properties of $\quad 57,5^{8}$

Silver ion, propertics of . . . . 58

Silver nitrate, standard solutions of . . . . . . . . . . $\mathrm{I} 66$

Soda, alkaline value of . . . II6

Sodium carbonate, primary standard . . . . . . II $3, \mathrm{II}_{4}$

Sodium chloride, determination of chlorine in . . . . . 55, I68 primary standard . . . . 166 purification of . . . . . 167

Sodium hydroxide, standard solutions of . . . . . . . . II2

Sodium oxalate, primary standard $\quad{ }^{32}$

Sodium thiosulphate, standard solutions of $I 5 I, I 53, I 6 I, I 62, I 64$

Solubility, effect of size of particles on . . . . . . . . 22

Solubility product . . . . 28

Solution tension . . . . . . 28 electrolytic . . . . . . . 93

Solution of iron ores . . I 29, I.34, I35

Solution of manganese ores . . 146

Standardization, definition of . . 99 of hydrochloric acid . . . . II 2

of sodium hydroxide solution . I I 2

of dichromate solution . . . I 25

of ferrous ammonium sulphate solution. . . . . . . . 125

of iodine solution . . . . . I5I

of permanganate solution . . I3I

of silver nitrate solution . . . 166

of sodium thiosulphate solution

I5 I, I6r, I62

Standard solution, definition of $\cdot 99$

Starch, indicator . . . . I5O, I5I

Stibnite, determination of antimony in . . . . . . I 55

Suction, use of . . . . $\quad 32,34,35$

Sulphur, determination of . . 64,66
Temperature, correction for differences in . . . . . 46

Tension, solution . . . . . 28

electrolytic solution . . . . 93

Testing for complete precipitation 33

of washings . . . . . . . 33

Thiocyanate solutions, standard . $\quad$ I66

Titration, definition of . . . . 2

Transference of liquids . . . 42

Transfer pipettes . . . . . . 44

Triangles. . . . . . 4I, 42

Utilization of time . . . . . 4

Vacuum, use of . . . . 32, 34, 35

Valve, Contat-Göckel . 138, 139

Volt, definition of . . . . . 92

Volume, units of . . . . . . 47

Volumetric analysis, general discussion . . . . . . . . 99

fundamental reactions of . . . IOI

neutralization methods of . . I07

oxidation and reduction methods of . . . . . . . . . I 24

precipitation methods of . . . 165

Volumetric apparatus . . . 43

calibration of . . . . . 48

cleaning of . . . . . . 45

sources of error in the use of . . 44

Volumetric System, Advantages of . . . . . . 43, r05

Volumetric Work, General Remarks . . . . . . . 105

Wash bottles . . . . . 33

Washing of precipitates . . 22, 31, 33

theory of . . . . . . 36

Washings, testing of . . . . 33

Water, ionization of . . . . 107

Weighing . . . . . . 7,53

limits of error in . . . . . II

methods of . . . . . . 12

summary of . . . . . . 20

Weights, calibration of . . . 15 use and care of . . . . . . ro

Zimmermann-Reinhardt solution . $\quad$ I34 
RENEWED BOOKS ARE SUBJECT TO IMMEDIATE RECALL

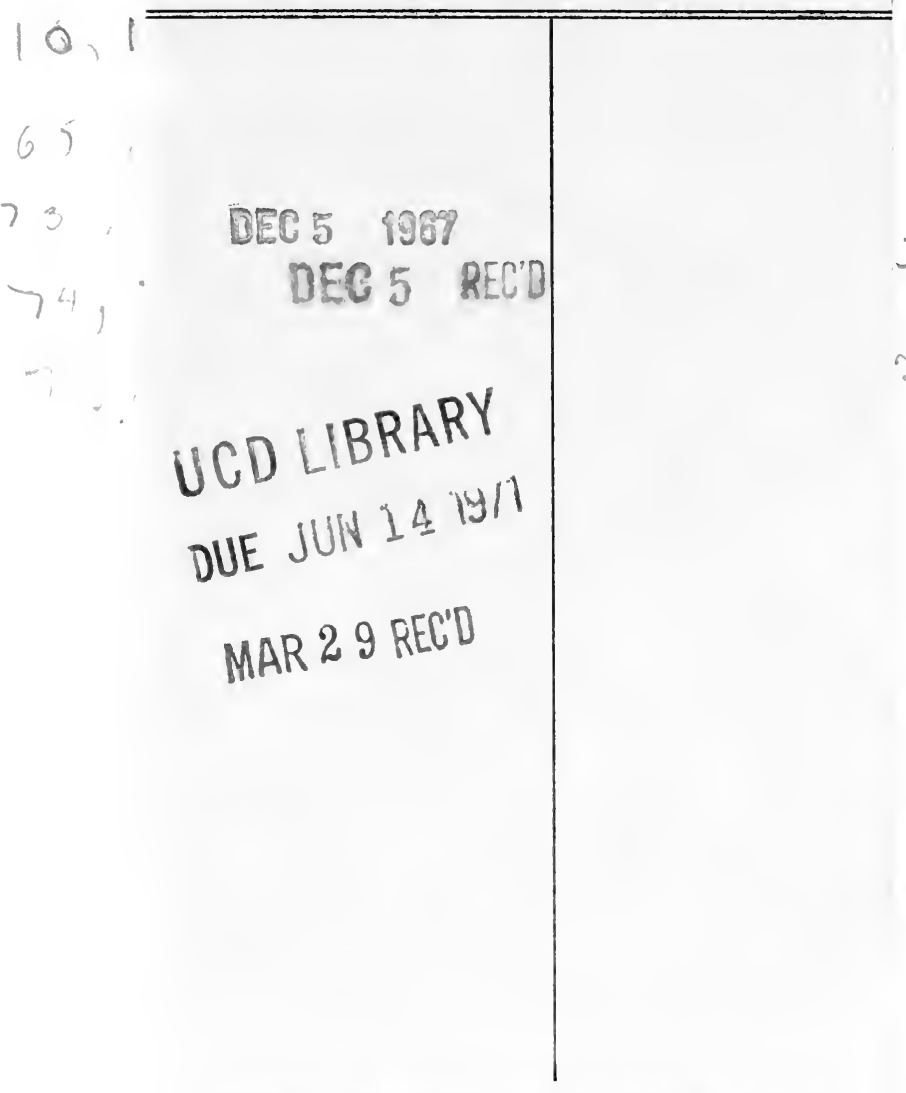

LIBRARY, UNIVERSITY OF CALIFORNIA, DAVIS

Book Slip-50m-12,'64 (F772s4)458 
Alumin

Antimo

Argon

Arsenic

Barium

Bismut]

Boron

Bromin

Cadmiu

Cæsium

Calciur

Carbon

Cerium

Chlorin

Chromit

Cobalt

Columbsume

Copper

Dysprosium

Erbium

Europium

Fluorine

Gadolinium

Gallium

Germanium

Glucinum

Gold

Helium

Holmium

Hydrogen

Indium

Iodine

Iridium

Iron

Krypton

Lanthanum

Lead

Lithium

Lutetium

Magnesium

Manganese

Mercury
Smith, G.M.

A course of in-

struction in

quantitative

chemical anal-

ysis.
QD101

S6

1921

UNIVERSITY OF CALIFORNIA DAVIS

96.0

I 44.3

20.2

58.68

222.4

I4. 008

190.9

16.000

106.\%

31.04

I 95.2

39.10

I 40.9

226.0

102.9

85.45

12u IOI.7

$\mathrm{Sa} \quad 150.4$

Sc $\quad 4.5 . \mathrm{I}$

Se $\quad 79.2$

Si 28.3

Ag $\quad 107.88$

$\mathrm{Na} \quad 23.00$

$\mathrm{Sr} \quad 87.63$

$\mathrm{S} \quad 32.06$

$\mathrm{Ta} \quad \mathrm{I} 8 \mathrm{I} .5$

Te $\quad 127.5$

$\mathrm{Tb} \quad \mathrm{I} 59.2$

$\mathrm{Tl} \quad 204.0$

Th 232.15

Tm $\quad$ I68.5

$\mathrm{Sn} \quad \mathrm{I} 8.7$

Ti 48.I

W $\quad$ I 84.0

Titanium

Tungsten

Uranium

Vanadium

Xenon

Ytterbium

Yttrium

Zinc

Zirconium

U 238.2

V 5 I.0

$\mathrm{Xe} \quad \mathrm{I} 30.2$

Yb $\quad$ I 73.5

Yt 89.33

Zn $\quad 65.37$

$\mathrm{Zr} \quad 90.6$ 


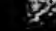

